\title{
Mobile Graphics
}

Siggraph Asia 2017 course

Marco Agus, KAUST \& CRS4

Enrico Gobbetti, CRS4

Fabio Marton, CRS4

Giovanni Pintore, CRS4

Pere-Pau Vázquez, UPC

November 2017

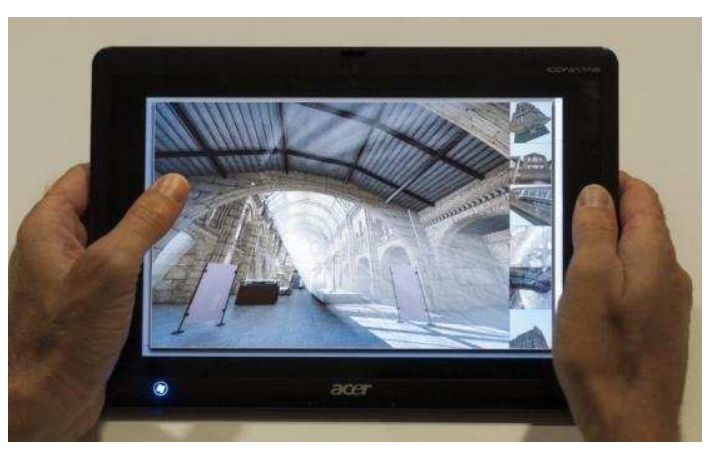

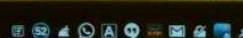

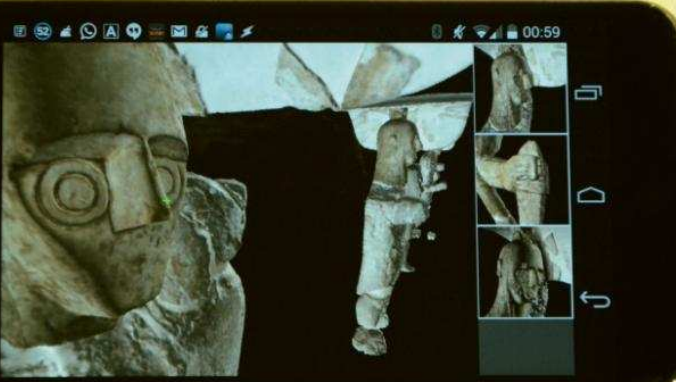

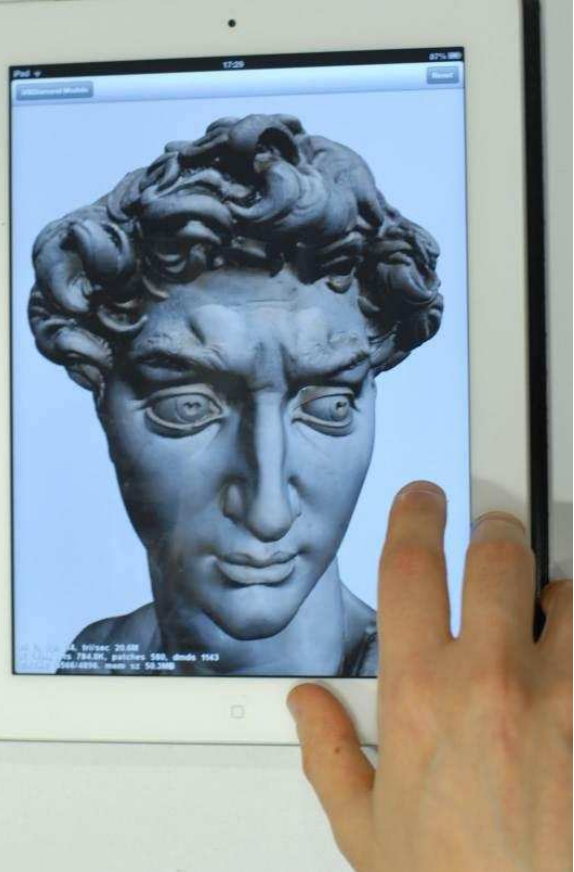


WELCOME TO THIS HALF-DAY COURSE! 


\section{Subject: Mobile Graphics}

- All you need to know to get an introduction to the field of mobile graphics:

- Scope and definition of "mobile graphics"

- Brief overview of current trends in terms of available hardware architectures and research apps built of top of them

- Quick overview of development environments

- Rendering, with focus on rendering massive/complex surface and volume models

- Capture, with focus on data fusion techniques 


\section{Speakers (in alphabetical order)}

- Marco Agus $(1,2)$

- Research Engineer at KAUST (Saudi Arabia)

(1) www.crs4.it/vic/

- Researcher at CRS4 (Italy)

- Enrico Gobbetti (1) - Organizer

- Director of Visual Computing at CRS4 (Italy)

(2) https://vcc.kaust.edu.sa

- Fabio Marton (1)

- Researcher at CRS4

- Giovanni Pintore (1)

(3) http://www.virvig.eu/

- Researcher at CRS4

- Pere-Pau Vázquez (3)

- Professor at UPC, Spain
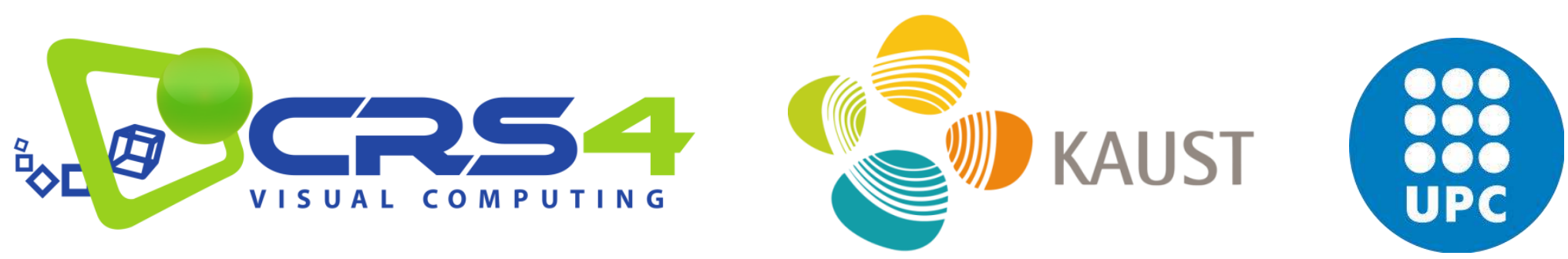


\section{Funding}

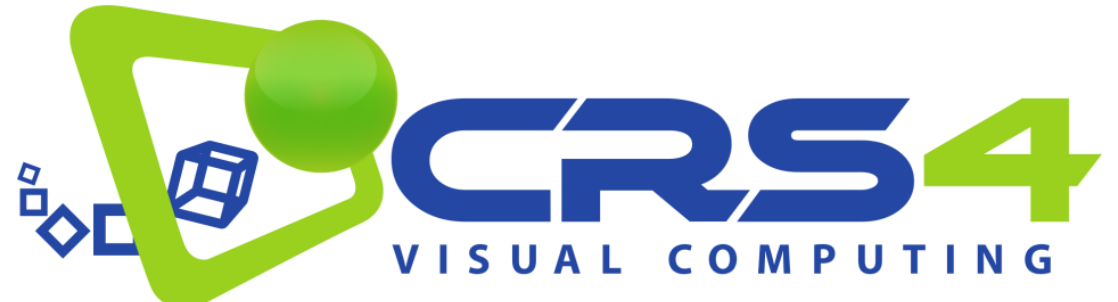

Center for Research, Development, and Advanced Studies in Sardinia, Italy

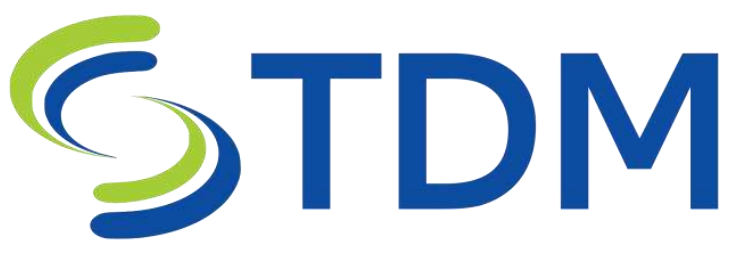

Project TDM RAS - POR FESR 2014-2020

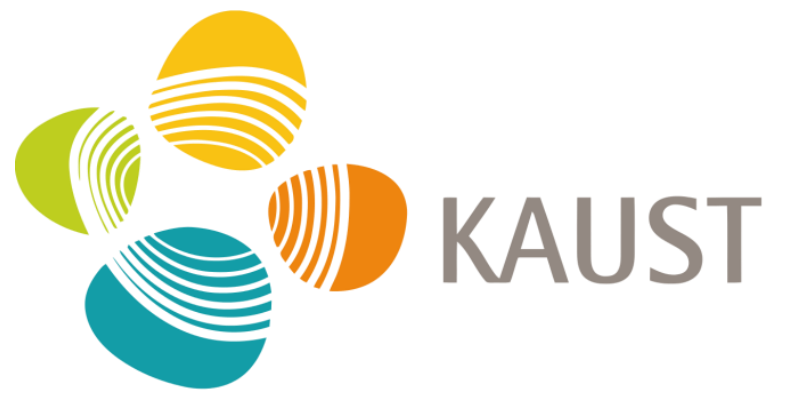

King Abdullah University of Science \& Technology, Saudi Arabia

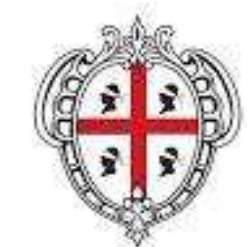

REGIONE AUTONOMA DE SARDIGNA REGIONE AUTONOMA DELLA SARDEGNA Projects VIGEC / VIDEOLAB

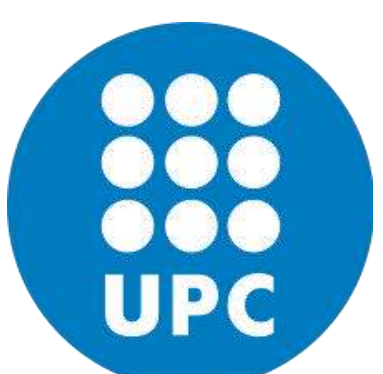

Polytechnic University of Catalonia, Spain 


\section{Schedule}

\begin{tabular}{|c|l|l|}
\hline $5^{\prime}$ & O. Introduction and outline & Enrico \\
\hline $15^{\prime}$ & 1. Evolution of Mobile Graphics & Marco \\
\hline $20^{\prime}$ & 2.1 Mobile Graphics Trends / Hardware & Pere-Pau \\
\hline $5^{\prime}$ & 2.2 Mobile Graphics Trends / Applications & Marco \\
\hline $15^{\prime}$ & 3. Graphics Development for Mobile Systems & Marco \\
\hline $5^{\prime}$ & 4.1 Scalable Mobile Visualization / Introduction & Enrico \\
\hline $30^{\prime}$ & 4.2 Scalable Mobile Visualization / Massive Meshes & Fabio \\
\hline $15^{\prime}$ & BREAK & - \\
\hline $5^{\prime}$ & 4.3 Scalable Mobile Visualization / Intro to complex lighting & Enrico \\
\hline $10^{\prime}$ & 4.4 Scalable Mobile Visualization / Lighting Precomputation & Fabio \\
\hline $20^{\prime}$ & 4.5 Scalable Mobile Visualization / Smart Shading & Pere-Pau \\
\hline $15^{\prime}$ & 4.6 Scalable Mobile Visualization / Volumes & Pere-Pau \\
\hline $10^{\prime}$ & 5.1 Mobile Metric Capture and Reconstruction / Introduction & Enrico \\
\hline $30^{\prime}$ & 5.2 Mobile Metric Capture and Reconstruction / Case studies & Gianni \\
\hline $15^{\prime}$ & 6. Closing and Q\&A & ALL \\
\hline
\end{tabular}


Next Session EVOLUTION OF MOBILE GRAPHICS 


\section{Part 1}

\section{Evolution of the mobile graphics world}

Marco Agus, KAUST \& CRS4 


\section{Mobile evolution (1/3)}

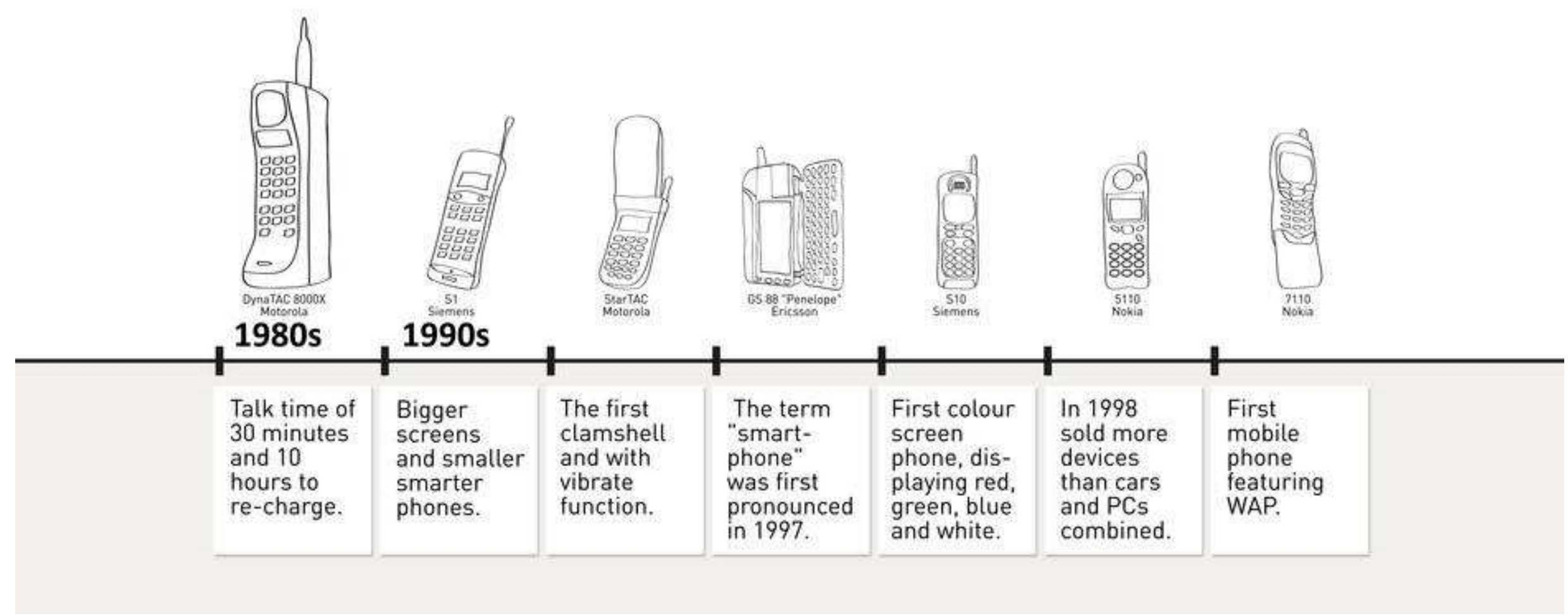




\section{Mobile evolution (1/3)}

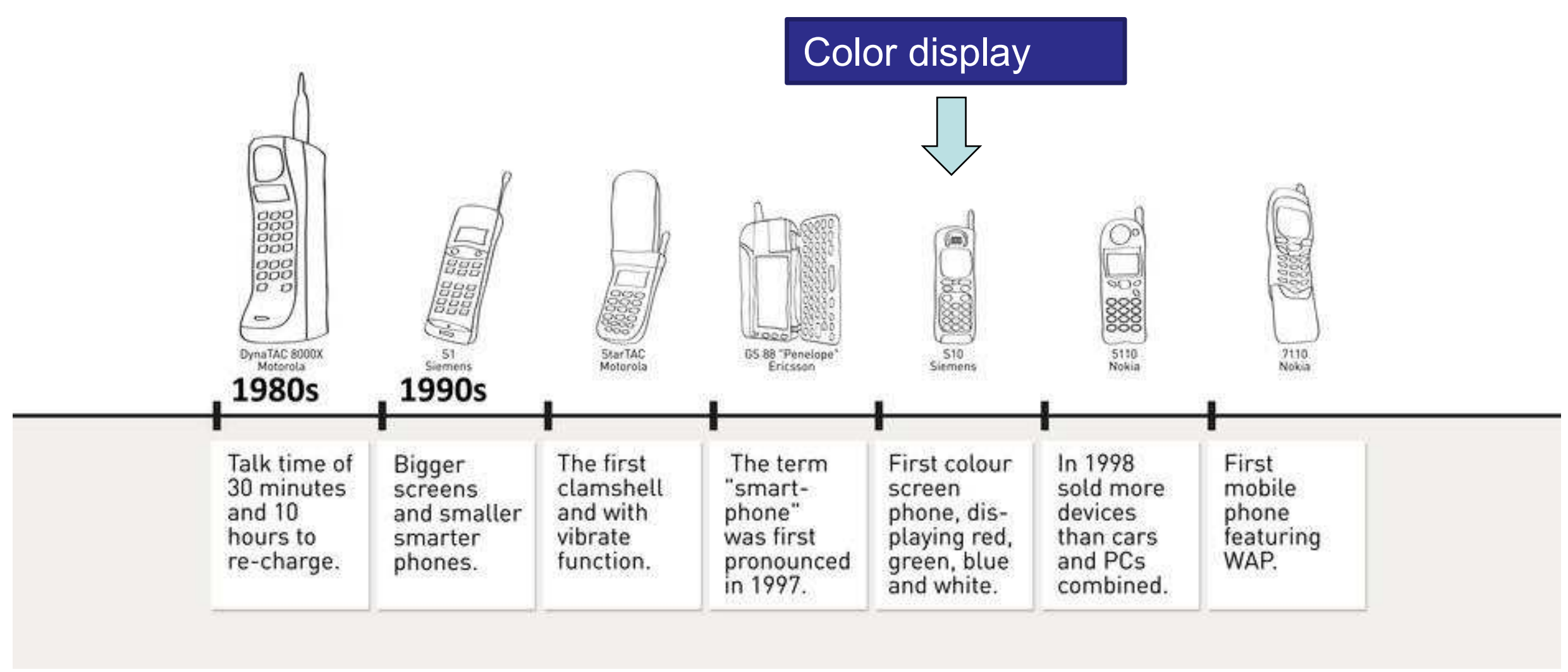




\section{Mobile evolution (2/3)}

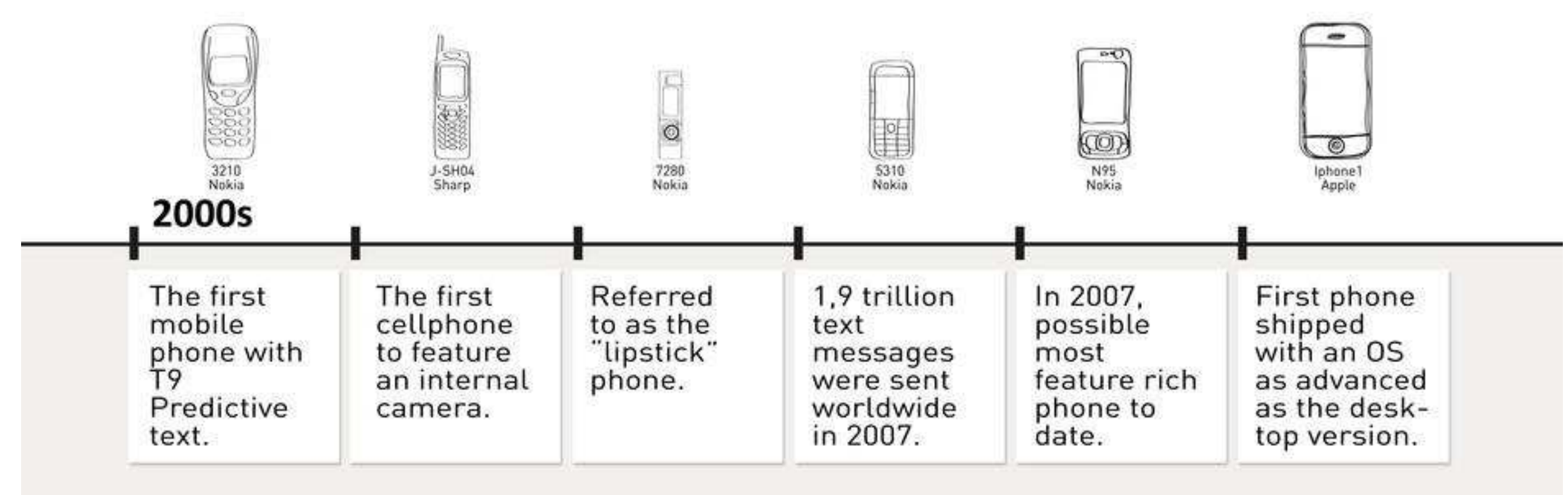




\section{Mobile evolution (2/3)}

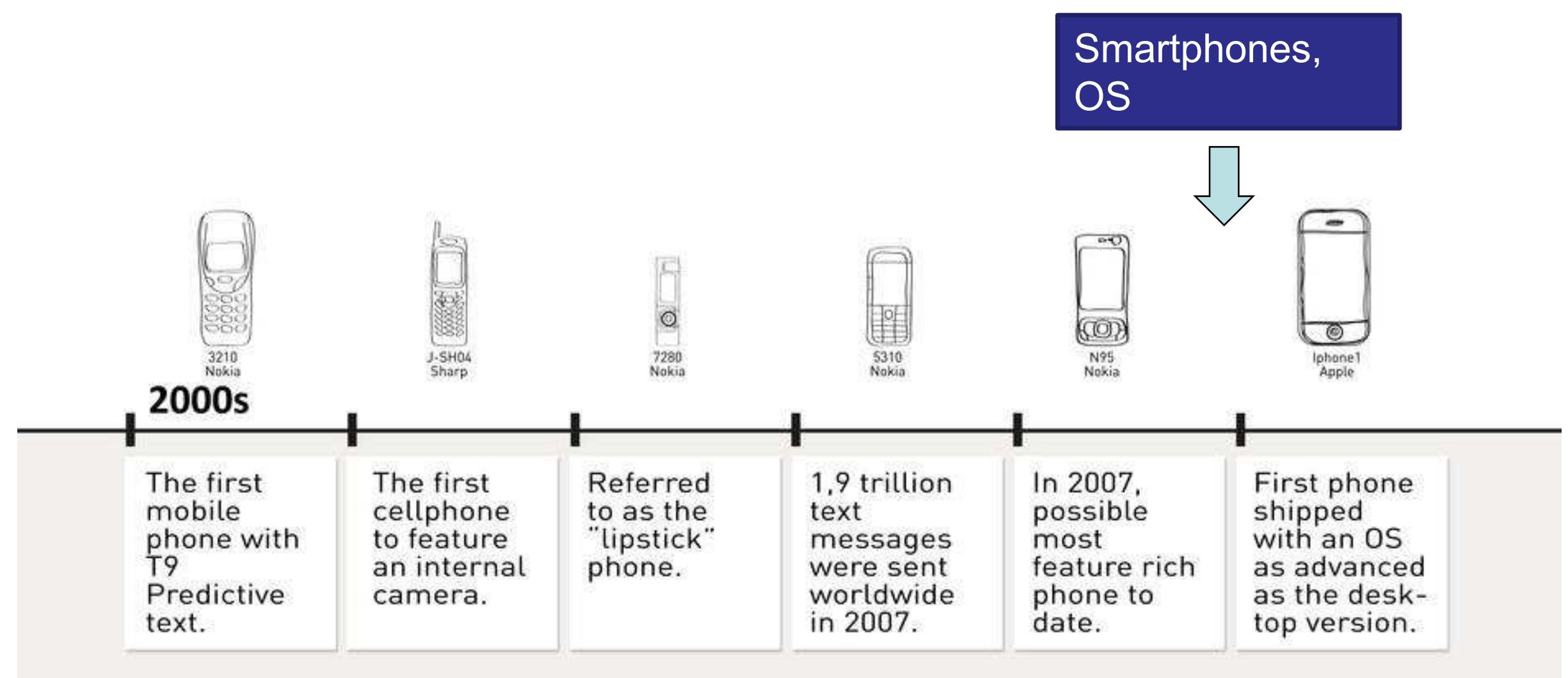




\section{Mobile evolution ( $3 / 3)$}

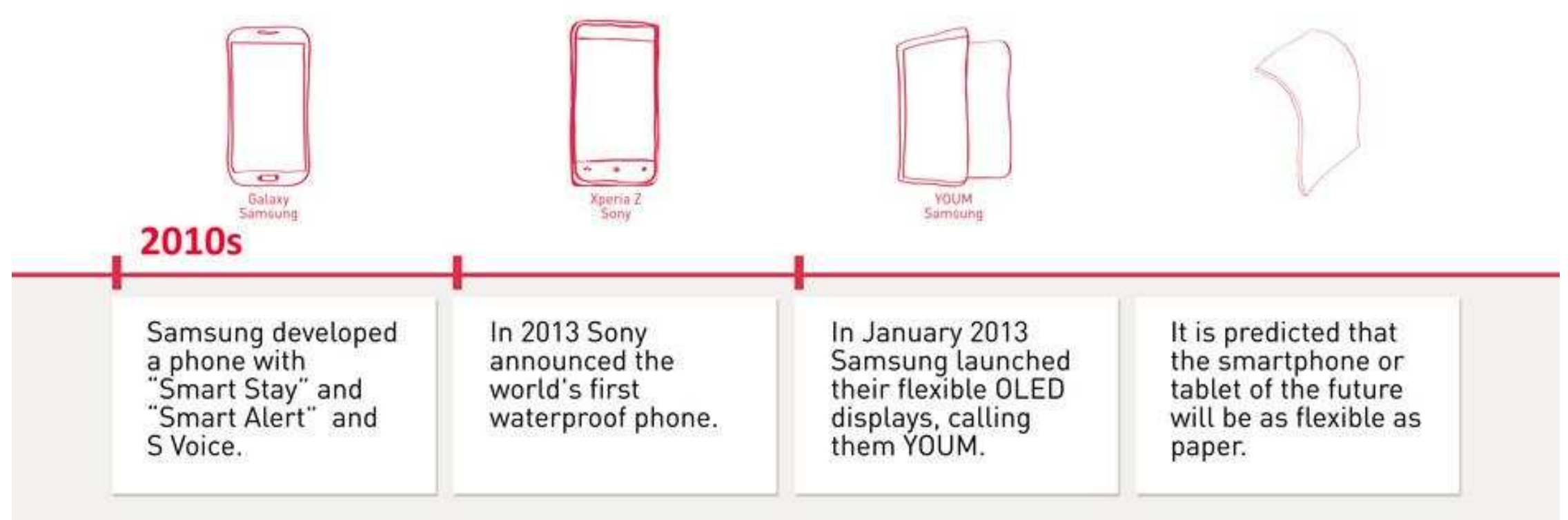




\section{Mobile evolution ( $3 / 3)$}

\section{High resolution}

displays
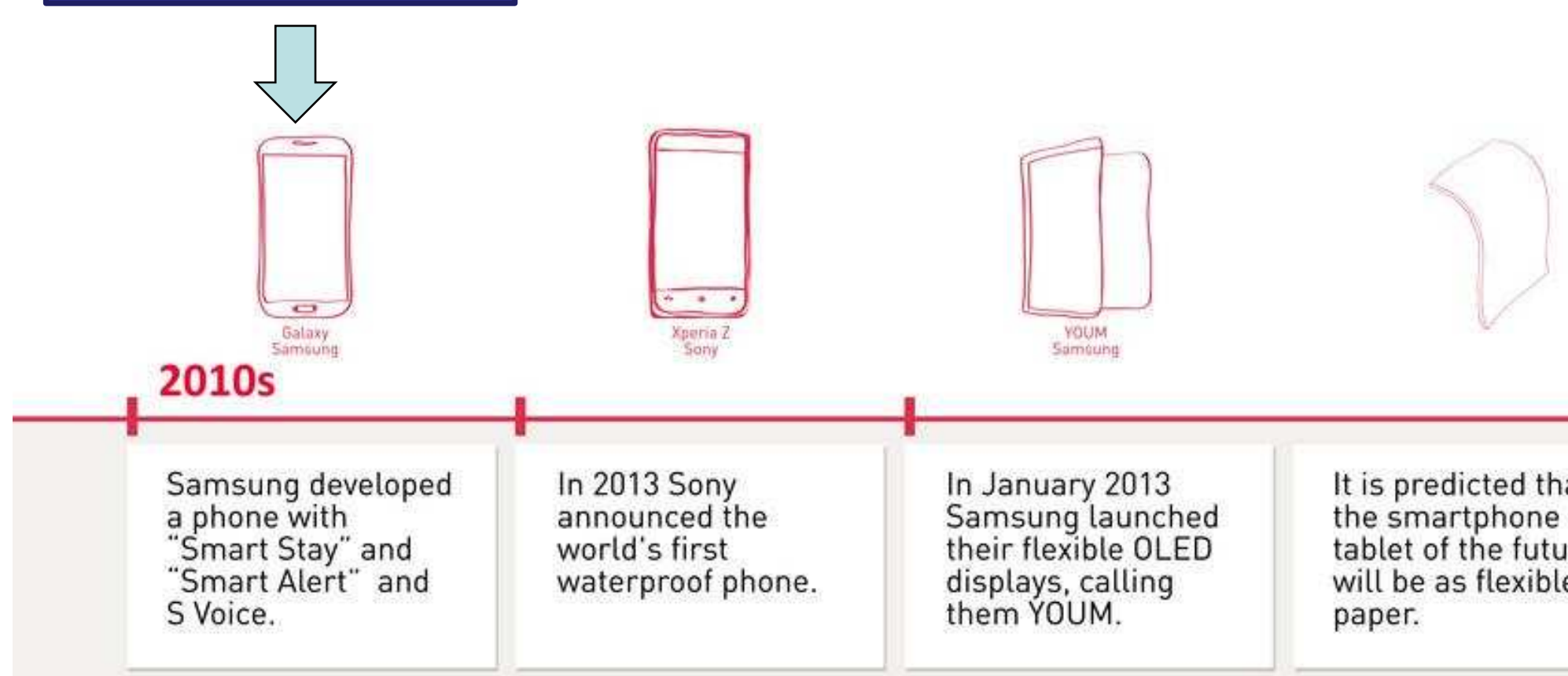

In 2013 Sony

announced the

world's first

waterproof phone.

In January 2013

Samsung launched

their flexible OLED

displays, calling

them YOUM.

It is predicted that

the smartphone or

tablet of the future

will be as flexible as

paper. 


\section{Mobile evolution ( $3 / 3)$}

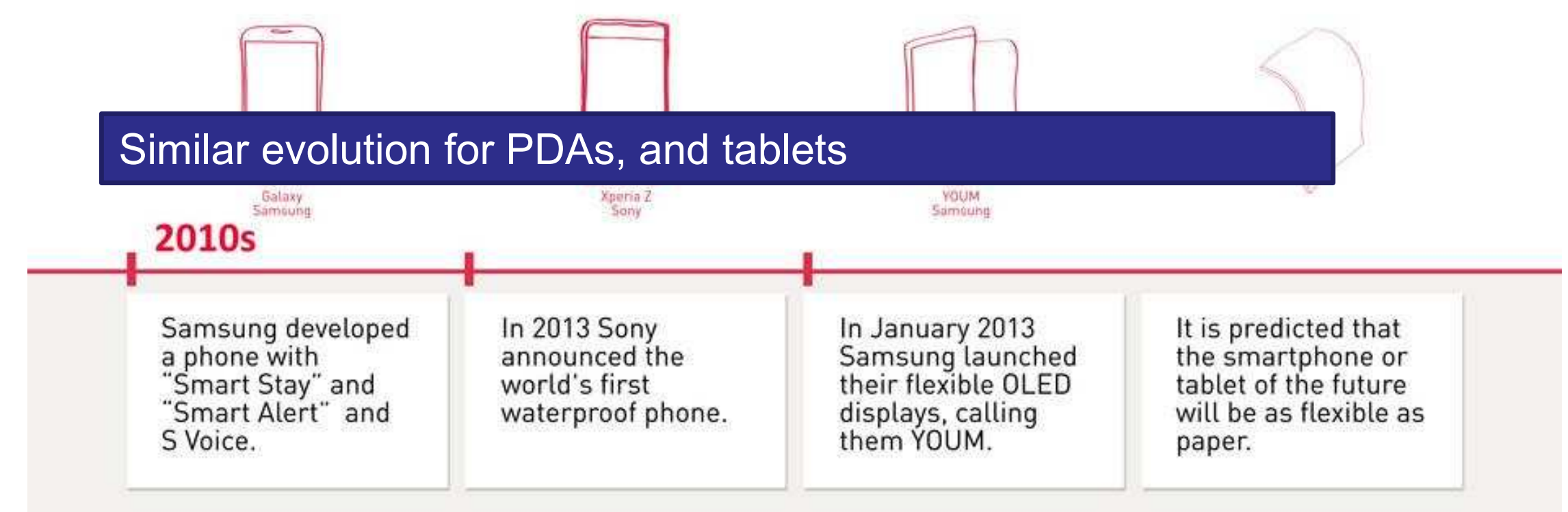




\section{Mobile connectivity evolution}

- Bandwidth is doubling every 18 months

- Mobile internet users overcame desktop internet users

- 2017 smartphone traffic expected at

2.7 GB per person per month

Mobile Internet Subscriptions to Reach 6.4 Billion by 2019

Number of mobile internet device subscriptions worldwide (in billions)

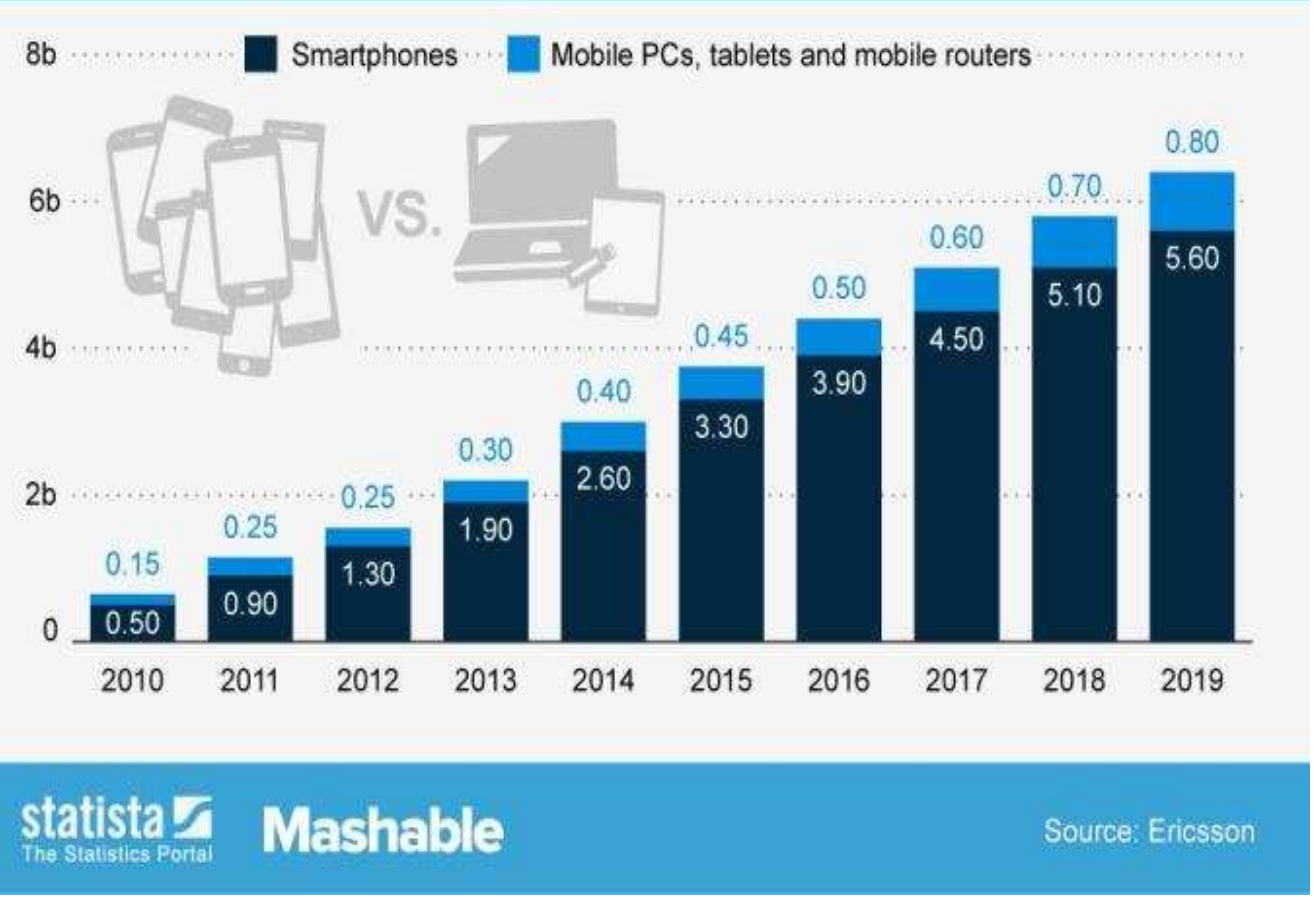

(C) www.statista.com 


\section{Displays and User Interface}

- Before 2007 - old days

- PDA $\rightarrow$ Palm OS/ Windows Pocket / Windows CE

- Stylus interaction (touch screens at early stages)

- Touch era

- 2007 - iOS /iPhone

- 2008 - Android / HTC Dream or G1

- Touch-enabled devices (no stylus required)

- Nowadays

- Wearables $\rightarrow<2$ "

- Smartphones $\rightarrow$ 3-6"

- Tablets $\rightarrow>7-10$ "

- DLP projectors integrated 


\section{Display characteristics}

\begin{tabular}{|c|c|c|c|c|c|c|c|c|}
\hline & Application & $100 \mathrm{PPI}$ & $150 \mathrm{PPI}$ & 200 PPI & $250 \mathrm{PPI}$ & $300 \mathrm{PPI}$ & $400 \mathrm{PPI}$ & 500 ррі \\
\hline $20 \mathrm{~cm}$ & $\begin{array}{l}\text { Smart } \\
\text { Phone }\end{array}$ & $\begin{array}{l}3.5^{\prime \prime} 400 \times 234 \\
(132 \mathrm{PPI})\end{array}$ & $\begin{array}{l}3.5^{\prime \prime} 480 \times 32 \mathrm{~d} \\
(164 \mathrm{PPI})\end{array}$ & & $\begin{array}{l}3.5^{\prime \prime} 800 \times 480 \\
(266 \mathrm{PPI})\end{array}$ & $\begin{array}{l}3.5^{\prime \prime} \\
960 \times 640 \\
(326 \mathrm{PPI})\end{array}$ & $\begin{array}{l}3.5^{\prime \prime} / 3.7^{\prime \prime} \\
1280 \times 800 \\
(400+\mathrm{PPI})\end{array}$ & $\begin{array}{l}5.1 " / 5.5 " \\
2560 \times 1440 \\
(>500 \mathrm{PPI})\end{array}$ \\
\hline \multirow[t]{3}{*}{$30 \mathrm{~cm}$} & \multirow[t]{3}{*}{ Tablet PC } & $\begin{array}{l}7 " 800 \times 480 \\
(133 \mathrm{PPI})\end{array}$ & $\begin{array}{l}7^{*} 1024 \times 600 \\
(169 \mathrm{PPI})\end{array}$ & $\begin{array}{c}7 " 1280 \times 800 \\
1366 \times 768 \\
(215 \mathrm{PPI})\end{array}$ & & & & \\
\hline & & $\begin{array}{l}9.7^{\prime \prime} 1024 \times 768 \\
(132 \mathrm{PPI})\end{array}$ & & $\begin{array}{l}9.7^{\prime \prime} \\
1600 \times 1200 \\
(206 \mathrm{PPI})\end{array}$ & $\begin{array}{l}9.7^{\prime \prime} \\
2048 \times 1536 \\
(264 \mathrm{PPI})\end{array}$ & & & \\
\hline & & $\begin{array}{l}10.1^{\prime \prime} 1024 \times 600 \\
(118 \mathrm{PPI})\end{array}$ & $\begin{array}{l}10.1^{n} \\
1280 \times 800 \\
1366 \times 768 \\
(150 \mathrm{PPI})\end{array}$ & $\begin{array}{l}10.1^{\prime \prime} \\
1920 \times 1080 \\
1920 \times 1200 \\
(210 \mathrm{PPI})\end{array}$ & $\Rightarrow$ & $\begin{array}{l}10.1^{\prime \prime} \\
2560 \times 1600 \\
(300 \text { PPI) }\end{array}$ & $\begin{array}{l}10.1^{\prime \prime} \\
3840 \times 2160 \\
(438 \mathrm{PPI})\end{array}$ & \\
\hline $40 \mathrm{~cm}$ & Mini-Note & $\begin{array}{l}10.1^{\prime \prime} 1024 \times 600 \\
(118 \mathrm{PPI})\end{array}$ & & & & & & \\
\hline $50 \mathrm{~cm}$ & $\begin{array}{l}\text { Notebook } \\
\text { PC }\end{array}$ & $\begin{array}{l}15.6^{\prime \prime} 1366 \times 768 \\
14.0^{\prime \prime} 1366 \times 768 \\
(110 \mathrm{PPI})\end{array}$ & & & & & & \\
\hline $60 \mathrm{~cm}$ & LCD MNT & $\begin{array}{l}21.5^{\prime} 1920 \times 1080 \\
(100 \mathrm{PPI})\end{array}$ & & & & & & \\
\hline
\end{tabular}




\section{Chip evolution (1/2)}

\section{Scalable Mobile Processor Evolution}

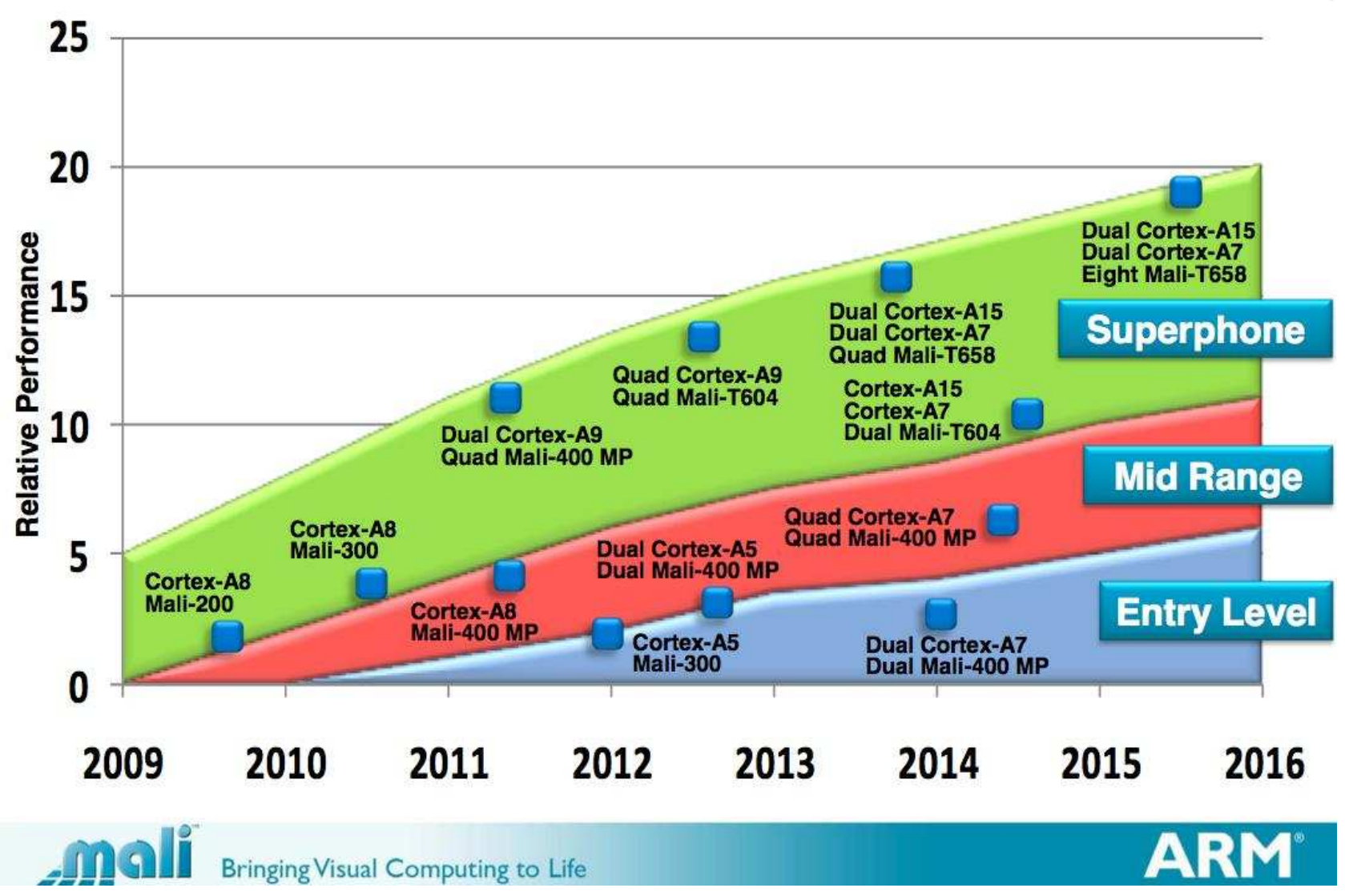

(C) ARM 


\section{Chip evolution (2/2)}

\section{The killer mobile processors ${ }^{\text {TM }}$}

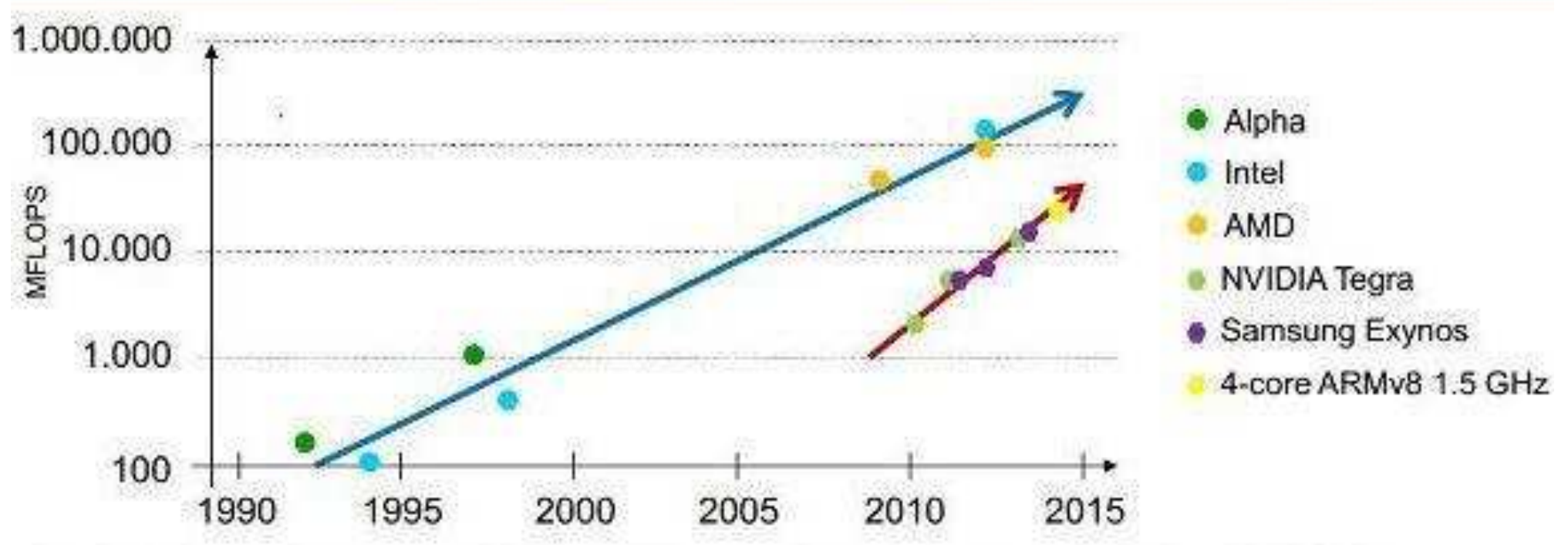

- Microprocessors killed the Vector supercomputers

- They were not faster ...

- ... but they were significantly cheaper and greener

- History may be about to repeat itself ....

- Mobile processor are not faster ...

- ... but they are significantly cheaper
(C) Rajovic, N., Carpenter, P., Gelado, I., Puzovic, N., \& Ramirez, A. (2013). Are mobile processors ready for HPC?. In Supercomput. 


\section{Scenario}

- Modern smartphones (tablets) are compact visual computing powerhouses

- DIFFUSION: more than $\mathbf{4 . 6}$ billion mobile phone subscriptions

- [Ellison 2010]

- NETWORKING: High speed internet connection (typical 1GB/month plan)

- 3G - < 0.6-3Mbps 100KB/s - 400KB/s (latency 100-125ms)

$-4 \mathrm{G}-<3-10 \mathrm{Mbps} \sim 400 \mathrm{~KB} / \mathrm{s}-1 \mathrm{MB} / \mathrm{s}$ (latency $\sim 60-70 \mathrm{~ms}$ )

- 5G - 1Gbps (from 2016?)

- MEMORY: Increasing RAM and storage space

- RAM 1-3GB

- Storage 8-64GB

- COMPUTING: Increasing processing power

- CPU 4-8 core @ 2.5Ghz

- GPU 72-192 cores ( ALUs) 


\section{Scenario}

- More than 4.6 billion mobile phone subscriptions

- [Ellison 2010]

- High speed internet connection (typical $1 \mathrm{~GB}$ /month plan)

- 3G - <0.6-3Mbps $\sim 100 \mathrm{~KB} / \mathrm{s}-400 \mathrm{~KB} / \mathrm{s}$

$-4 \mathrm{G}-<3-10 \mathrm{Mbps} \sim 400 \mathrm{~KB} / \mathrm{s}-1 \mathrm{MB} / \mathrm{s}$

- Increasing RAM and storage space

- RAM 1-3GB

- Storage 8-64GB

- Increasing processing power

- CPU 4-8 core @ 2.5Ghz

- GPU 72-192 cores ( ALUs) 


\section{Where are we going?}

- Powerful devices for acquiring, processing and visualizing information

- Accessibility of information (anybody, any time, anywhere)

- Immense potential (integration of acquisition, processing, visualization, cloud computing, and collaborative tasks) 
Next Session

MOBILE GRAPHICS TRENDS: HARDWARE ARCHITECTURES \& APPLICATIONS 


\title{
Part 2.1
}

\section{Mobile Graphics Trends:}

\author{
Hardware Architectures
}

\section{Pere-Pau Vázquez, UPC}




\section{Architectures (beginning 2015)}
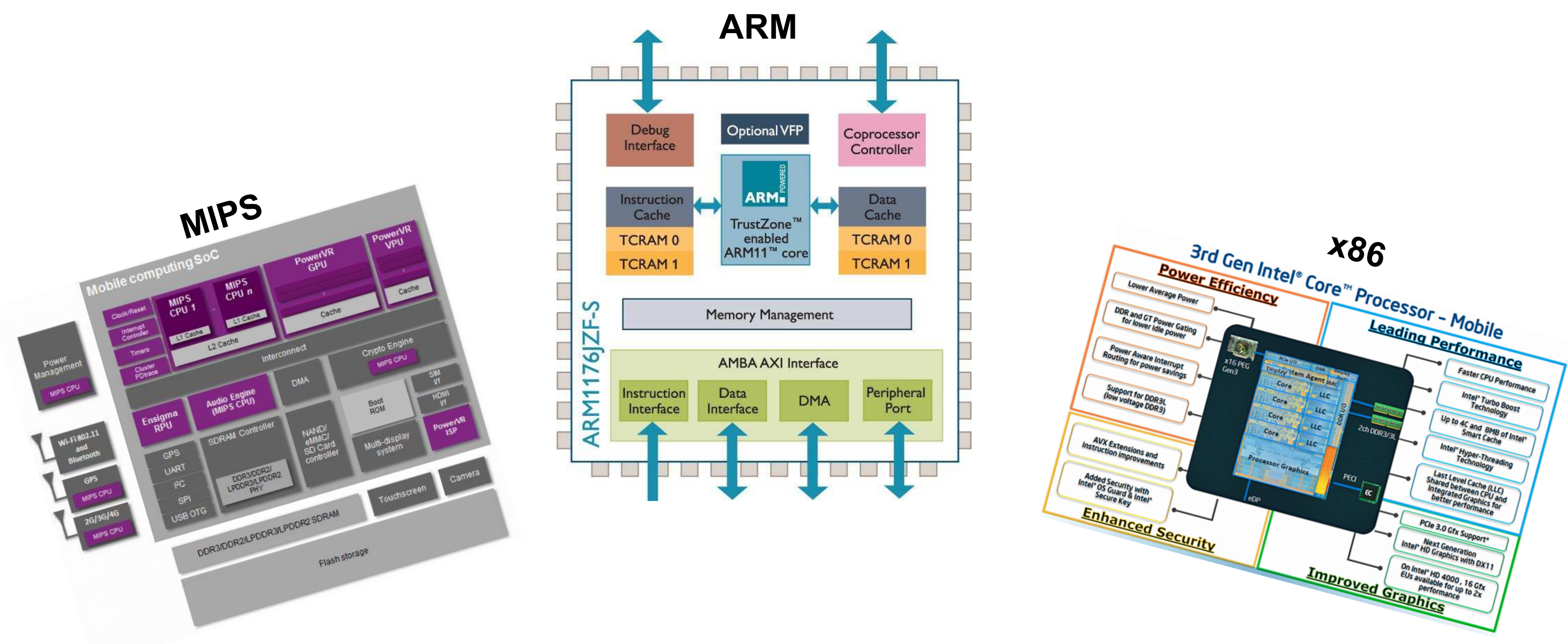


\section{Architectures}

- x86 (CISC 32/64bit)

- Intel Atom Z3740/Z3770, X3/X5/X7

- AMD Amur / Styx (announced)

- Present in few smartphones, more common in tablets

- Less efficient

- ARM

- RISC 32/64bit

- With SIMD add-ons

- Most common chip for smartphones

- More efficient \& smaller area

\section{- MIPS}

- RISC 32/64bit

- Including some SIMD instructions

- Acquired by Imagination, Inc. @2014 


\section{Architectures - RISC vs. CISC but...}

- CISC (Complex Instruction Set Computer)

- Fast program execution (optimized complex paths)

- Complex instructions (i.e. memory-to-memory instructions)

- RISC (Reduced Instruction Set Computer)

- Fast instructions (fixed cycles per instruction)

- Simple instructions (fixed/reduced cost per instruction)

- FISC (Fast Instruction Set Computer)

- Current RISC processors integrate many improvements from CISC: superscalar, branch prediction, SIMD, out-of-order

- Philosophy $\rightarrow$ fixed/reduced cycle count/instr

- Discussion (Post-RISC):

- http://archive.arstechnica.com/cpu/4q99/risc-cisc/rvc-5.html 


\section{Landscape has changed a bit...}

\section{- Status by 2014-2015:}

-Intel Atom X3/X5/X7 announced (March 2015)

-AMD announces Amur / Styx (20nm, Oct. 2014)

-Nvidia launches Tegra X1 (March 2015)

-ARM the only EU big technology company

- Imagination announces Furian (sub 14nm, March 2017) Imagination's chips are in iPhones \& iPads

\section{- Nowadays:}

-Intel quits mobile Apr/May 2016

-AMD cancels 20nm chips (Jul. 2015)

-Nvidia cancels Shield tablet (Aug. 2016)

-ARM acquired by Softbank (Sep. 2016)

-Apple tells Imagination that their IP will not be needed in 18-24 months (Apr. 2017) 


\section{Architectures (nowadays)}
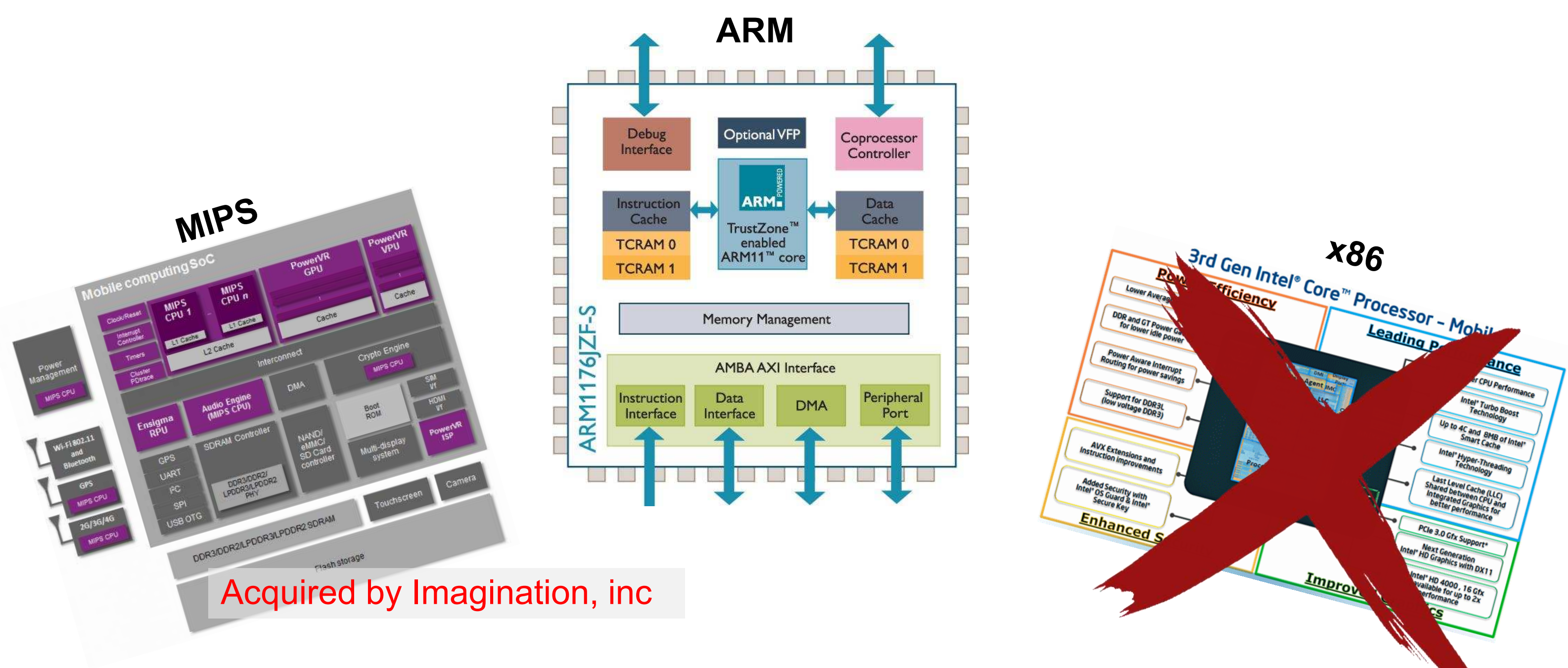


\section{Architectures - ARM}

- ARM Ltd.

- RISC processor (32/64 bit)

- IP (intellectual property) - Instruction Set / ref. implementation

- CPU / GPU (Mali)

- Licenses (instruction set OR ref. design)

- Instruction Set license -> custom made design (SnapDragon, Samsung in Galaxys, Apple in iPones \& iPads)

- Optimizations (particular paths, improved core freq. control,...)

- Reference design (Cortex A9, Cortex A15, Cortex A53/A57 ...)

- Licensees (instruction set OR ref. design)

- Apple, Qualcomm, Samsung, Nvidia, AMD, MediaTek, Amazon (through Annapurna Labs, Inc.)...

- Few IS licenses, mostly adopting reference design

\section{- Manufacturers}

- Contracted by Licensees

- GlobalFoundries, United Microelectronics, TSM... 


\section{Architectures - ARM...}

- Suppported on

- Android, iOS, Win Phone, Tizen, Firefox OS, BlackBerry, Ubuntu Phone, ...

- Biggest mobile market share

- Typically paired with mobile GPUs. Existing brands:

- Adreno 4x0/5x0 - Qualcomm

- PowerVR 8XE (Rogue) - Imagination

- Mali T8x0/G51/G71 - ARM

- General strategies:

- Cache coherence - week sequential code guarantees on multithreading!!

- Heavy dependence on compiler $\rightarrow$ optimize instruction scheduling

- Operation dependencies, loop unrolling, etc...

- Use SIMD extensions 


\section{Architecture types}

- High performance

- Premium smartphones \& tablets

- High area efficiency

- Medium-to-low smartphones

- Ultra-low power

- Smartwatches 


\section{Architectures}

Mobile GPU architecture trends 


\section{Graphics pipeline trends}

- Tiled rendering

- Data (texture) compression

- Other optimizations 


\section{Tiled Rendering}

- Immediate Mode Rendering (IMR)

- Tile-Based Rendering (TBR)

- Tile-Based Deferred Rendering (TBDR) 


\section{Architectures - GPU}

- Inmediate Mode Rendering (IMR)

- Geometry is processed in submission order

- High overdraw (shaded pixels can be overwritten)

- Buffers are kept in System Memory

- High bandwidth / power / latency

- Early-Z helps depending on geometry sorting

- Depth buffer value closer than fragment $\rightarrow$ discard

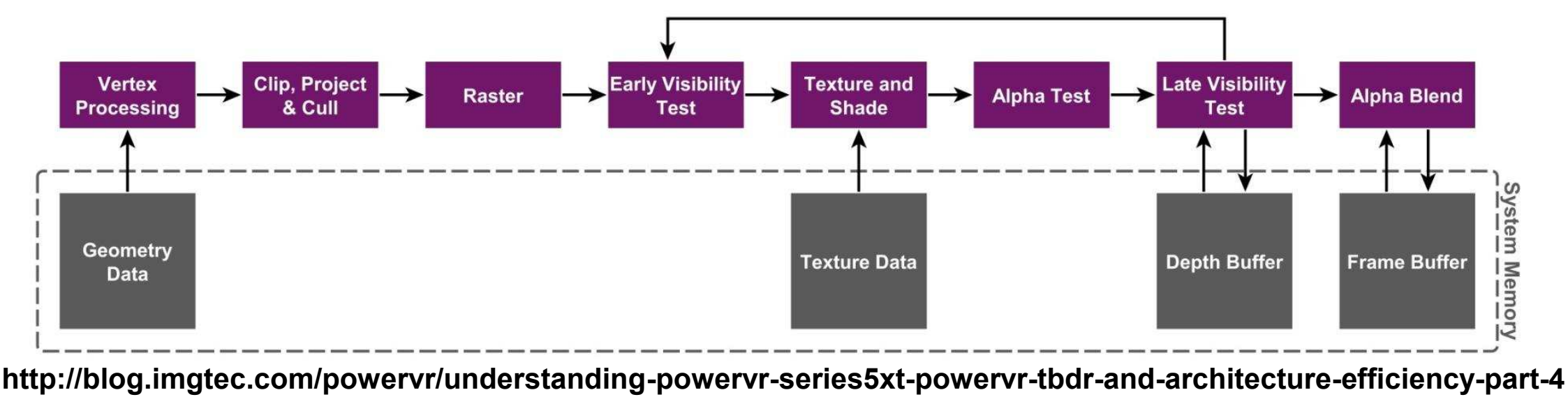




\section{Architectures - GPU}

\section{- Tile Based Rendering (TBR)}

\begin{tabular}{|c|c|c|c|}
\hline \multicolumn{3}{|c|}{ Screen } & \\
\hline \begin{tabular}{|l|l}
$(1,1)$ \\
\end{tabular} & $\mid(2,1)$ & $(3,1)$ & \\
\hline & & $(3,2)$ & \\
\hline & & & \\
\hline
\end{tabular}

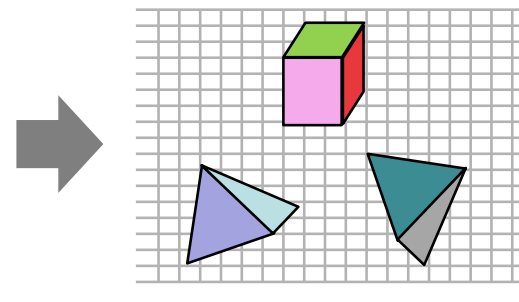

- Rasterizing per-tile (triangles in bins per tile) 16x16, 32×32

- Buffers are kept on-chip memory (GPU) - fast! $\rightarrow$ geometry limit?

- Triangles processed in submission order (TB-IMR)

- Overdraw (front-to-back -> early z cull)

- Early-Z helps depending on geometry sorting

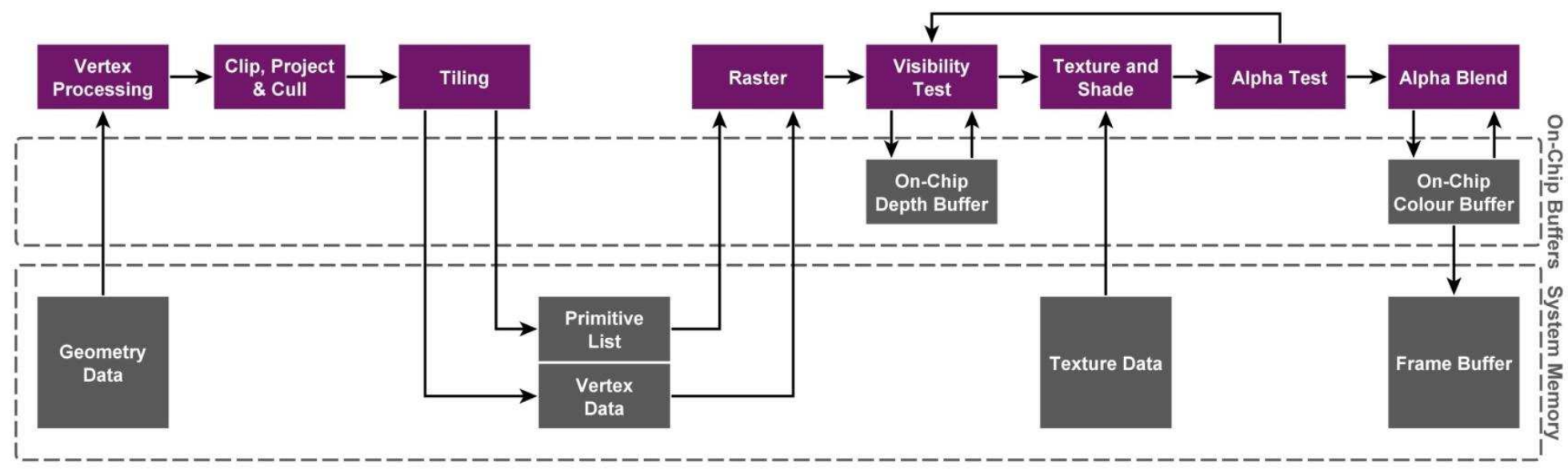

http://blog.imgtec.com/powervr/understanding-powervr-series5xt-powervr-tbdr-and-architecture-efficiency-part-4 


\section{Architectures - GPU}

\section{- Tile Based Deferred Rendering (TBDR)}

\begin{tabular}{|c|c|c|c|}
\hline \multicolumn{3}{|c|}{ Screen } & \\
\hline \begin{tabular}{|l}
$(1,1)$ \\
\end{tabular} & $(2,1)$ & $(3,1)$ & \\
\hline$(1,2$ & & $(3,2)$ & \\
\hline & & & \\
\hline
\end{tabular}

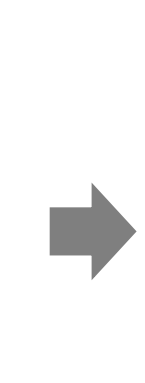

- Fragment processing (tex + shade) waits for Hidden Surface Removal

- Micro Depth Buffer - depth test before fragment submission

- whole tile $\rightarrow 1 \mathrm{frag} /$ pixel $:)$

\section{Limit: $\sim 100 \mathrm{Ktri}+$ complex shader}

- iPAD 2X slower than Desktop GeForce at HSR (FastMobileShaders_siggraph2011)

- Possible to prefetch textures before shading/texturing

- Hard to profile!!! Timing?

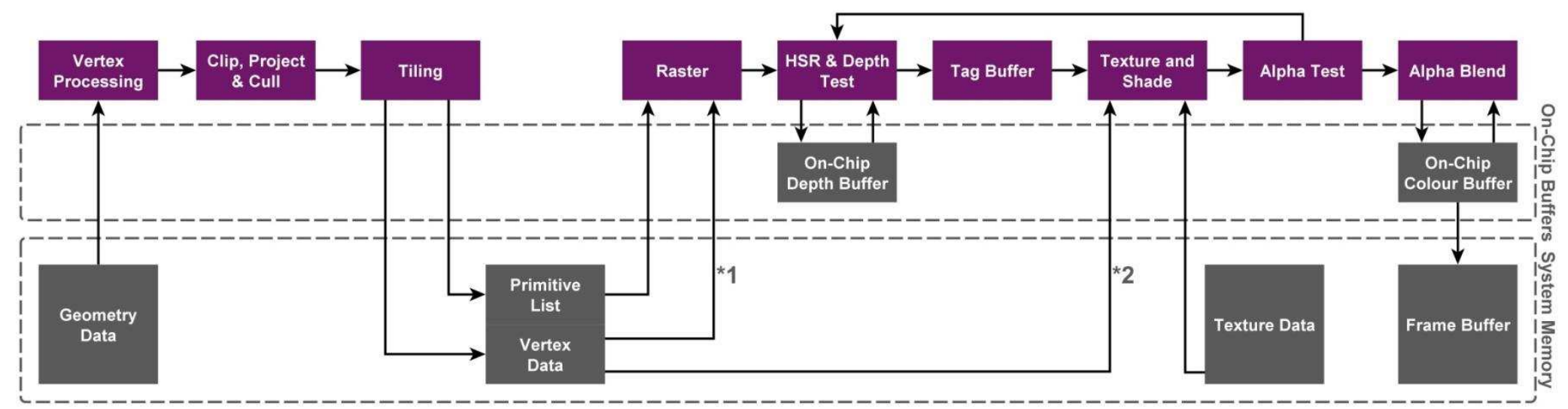

http://blog.imgtec.com/powervr/understanding-powervr-series5xt-powervr-tbdr-and-architecture-efficiency-part-4 


\section{Data/texture compression}

- ARM's Adaptive Scalable Texture Compression (ASTC) supported by most mobile GPU vendors

- ETC2/EAC standard compression OpenGL ES 3.0

- Compression hardware also present in display hardware

- Rendered images stored and transferred to the display in a compressed

- Saving bandwidth 


\section{Other optimizations}

- Deferred shading

- Primitive elimination

- Skipping updates to pixels that do not change

- ARM memory transaction elimination 


\section{Trends}

- Specific hardware for ray tracing

- Learning libraries \& hardware (e.g. Qualcomm's Fast CV, Nvidia's CUDA Deep Neural Network)

- Skipping updates to pixels that do not change

- ARM memory transaction elimination 


\section{Part 2.2}

\section{Mobile Graphics Trends: Applications}

\section{Marco Agus, KAUST \& CRS4}




\section{Applications}

- Wide range of applications

- Cultural Heritage

- Medical Image

- 3D object registration

- GIS

- Gaming

- VR \& AR

- Building reconstruction

- Virtual $\mathrm{HCl}$ 


\section{Mobile 3D interactive graphics}

- General pipeline similar to standard interactive applications

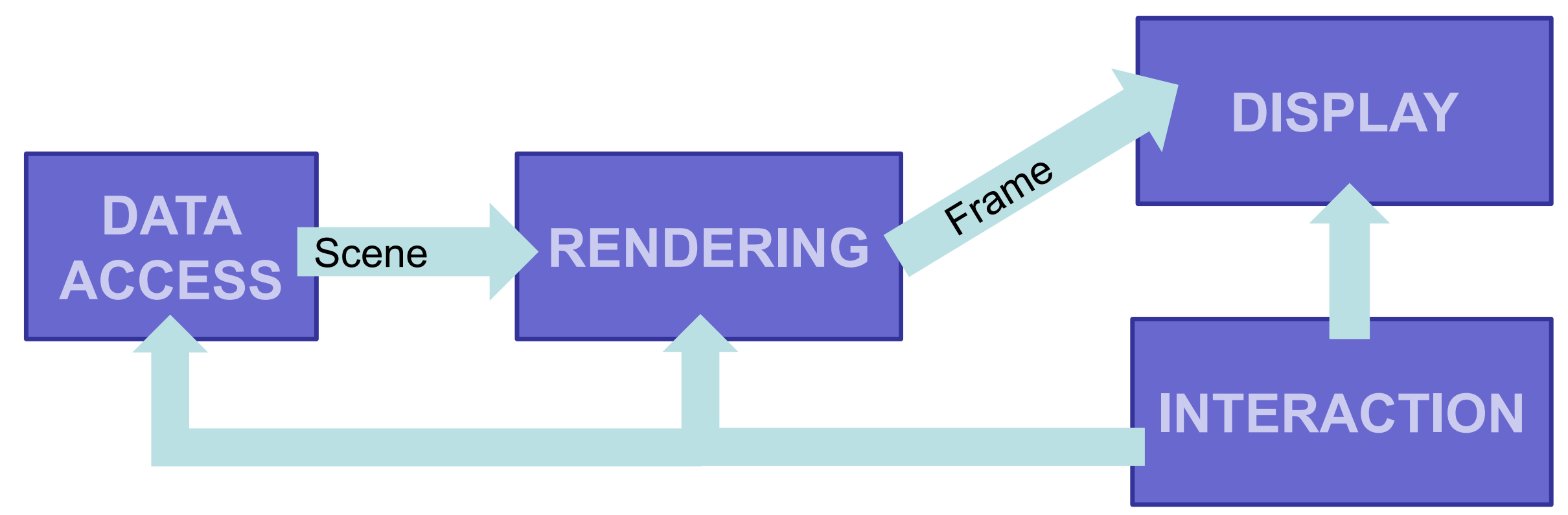




\section{Remote rendering}

- General solution since first PDAs

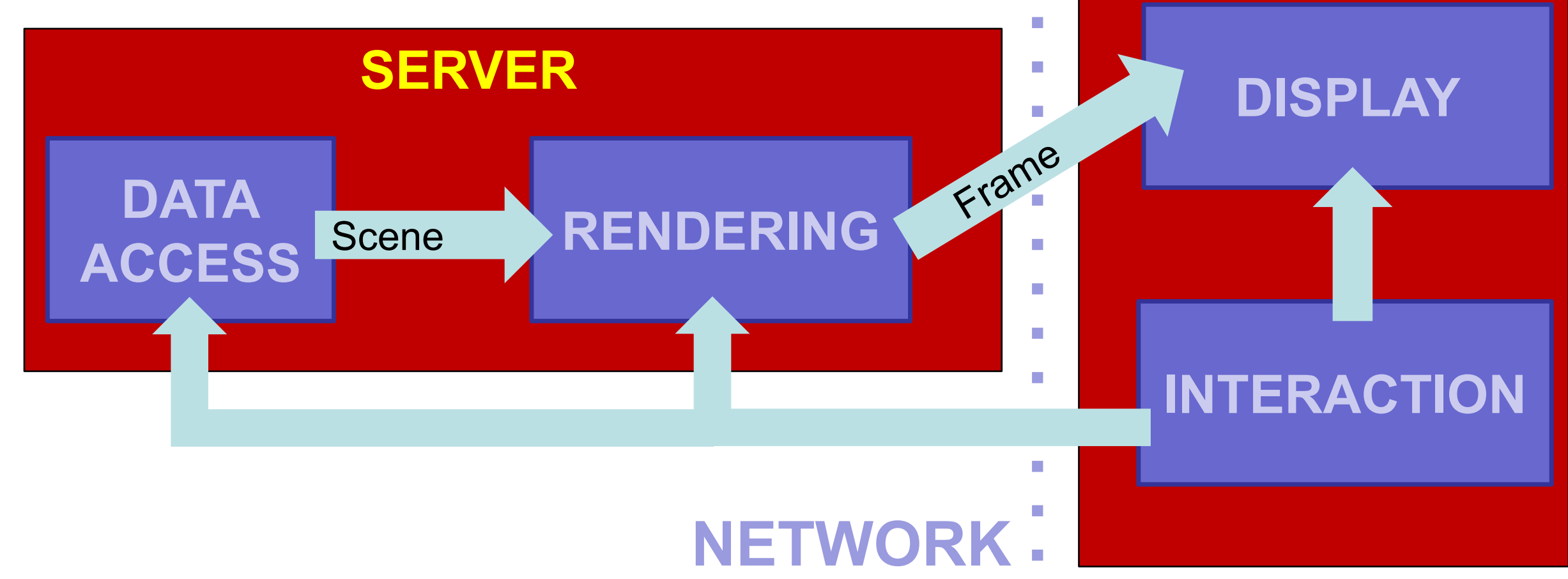




\section{Remote rendering}

- 3D graphics applications require intensive computation and network bandwidth

- electronic games

- visualization of very complex 3D scenes

- Remote rendering has long history and it is successfully applied for gaming services

- Limitation: interaction latency in cellular networks 


\section{Mixed Mobile/Remote rendering}

- As mobile GPUs progress...

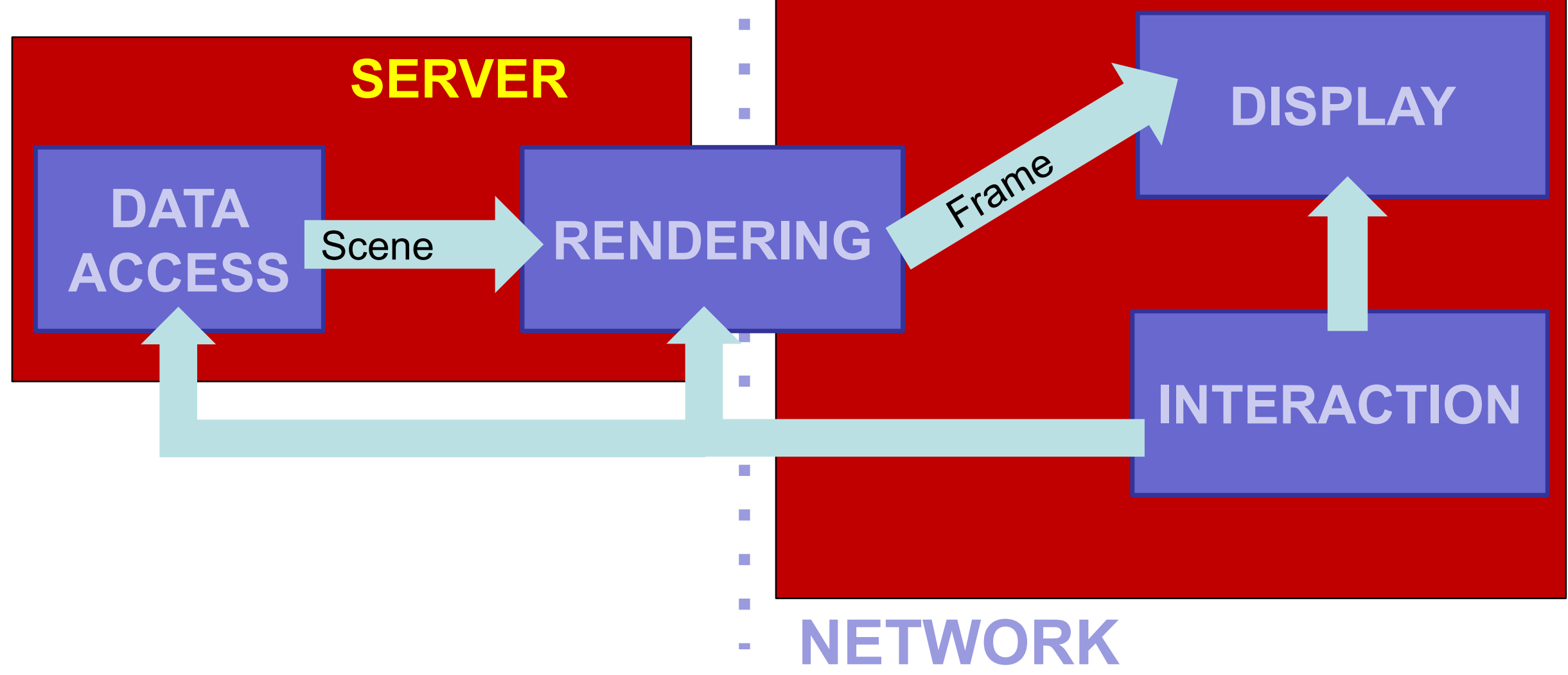




\section{Mixed Mobile/Remote rendering}

- Model based versus Image based methods

- Model based methods

- Original models

- Partial models

- Simplified models

Eisert and Fechteler. Low delay streaming of computer graphics (ICIP 2008)

Gobbetti et al. Adaptive Quad Patches: an Adaptive Regular Structure for Web Distribution and Adaptive Rendering of 3D Models. (Web3D 2012)

Balsa et al.,. Compression-domain Seamless Multiresolution Visualization of Gigantic Meshes on Mobile Devices (Web3D 2013)

- Couple of lines

Diepstraten et al., 2004. Remote Line Rendering for Mobile Devices (CGI 2004)

- Point clouds Duguet and Drettakis. Flexible point-based rendering on mobile devices (IEEE Trans. on CG \& Appl, 2004) 


\section{Mixed Mobile/Remote rendering}

- Model based versus Image based methods

- Model based methods

Point clouds
organized as
hierarchical grids.
Tested on PDAs

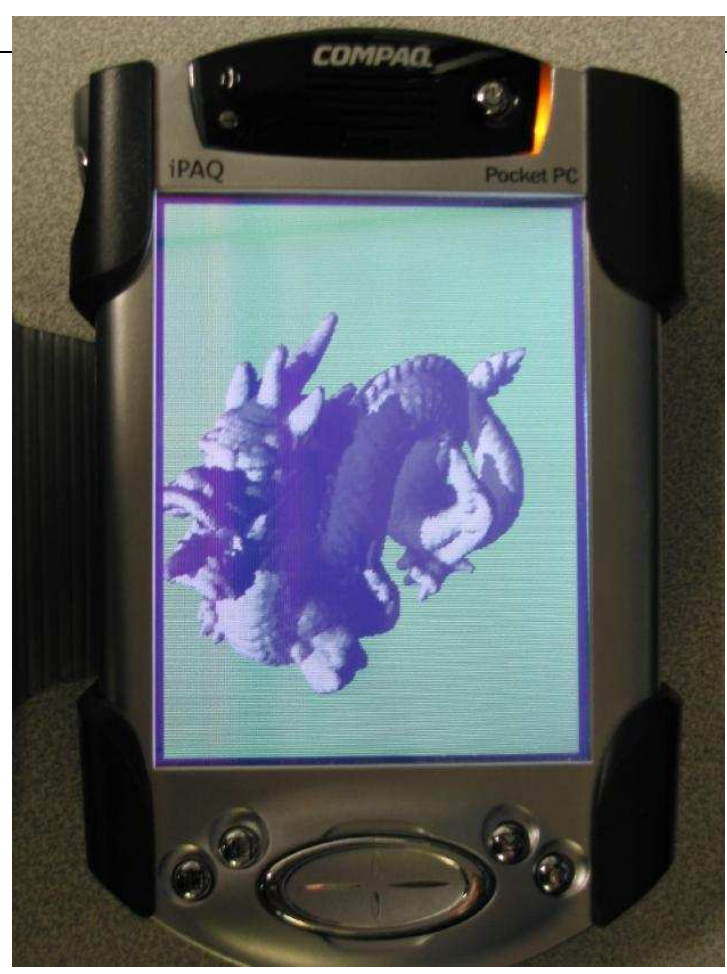

- Point clouds Duguet and Drettakis. Flexible point-based rendering on mobile devices (IEEE Trans. on CG \& Appl, 2004) 


\section{Mixed Mobile/Remote rendering}

- Model based versus Image based methods

- Model based methods

Transfer couple of 2D line primitives over the network, which are rendered locally by the mobile device

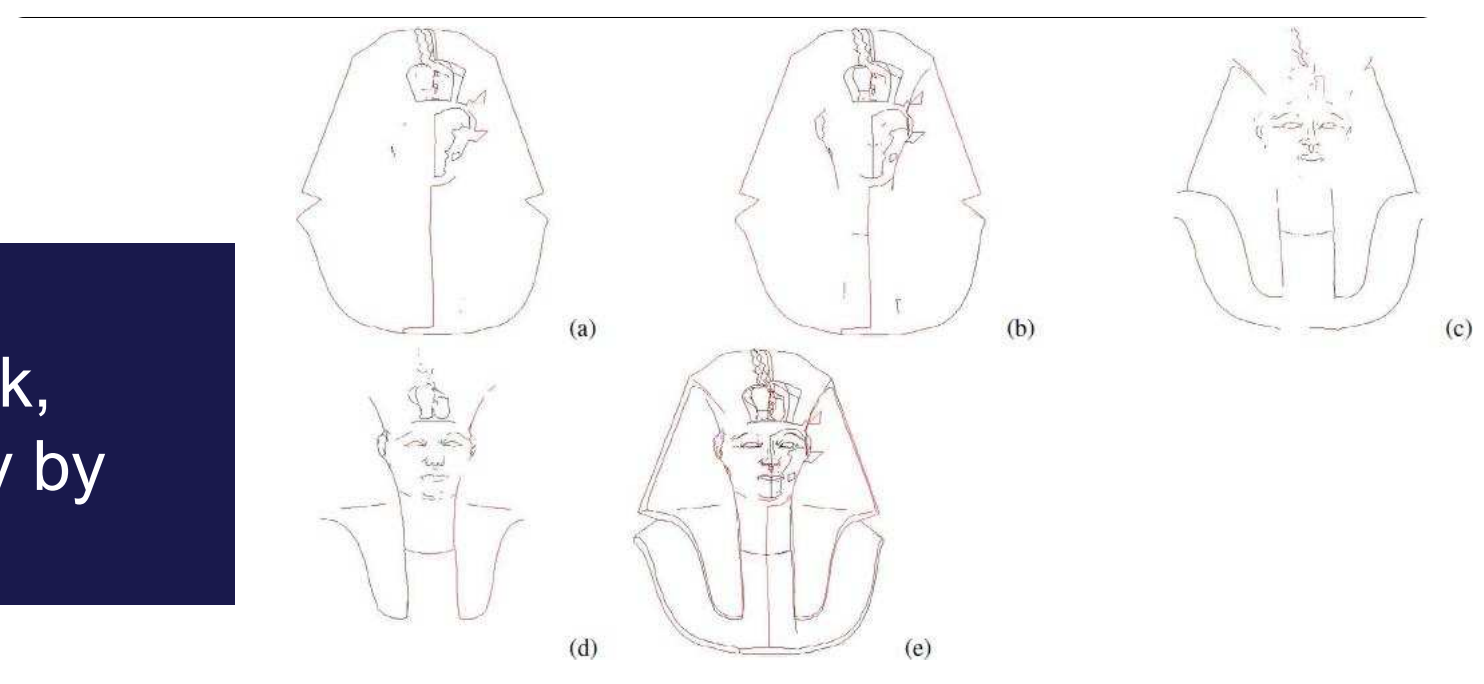

- Couple of lines Diepstraten et al., 2004. Remote Line Rendering for Mobile Devices (CGI 2004)

- Point clouds mobile devices (IEEE Trans. on CG \& Appl, 2004) 


\section{Mixed Mobile/Remote rendering}

- Model based versus Image based methods

- Model based methods

- Original models

\section{Eisert and Fechteler. Low delay streaming of computer} graphics (ICIP 2008)

graphics (ICIP 2008)
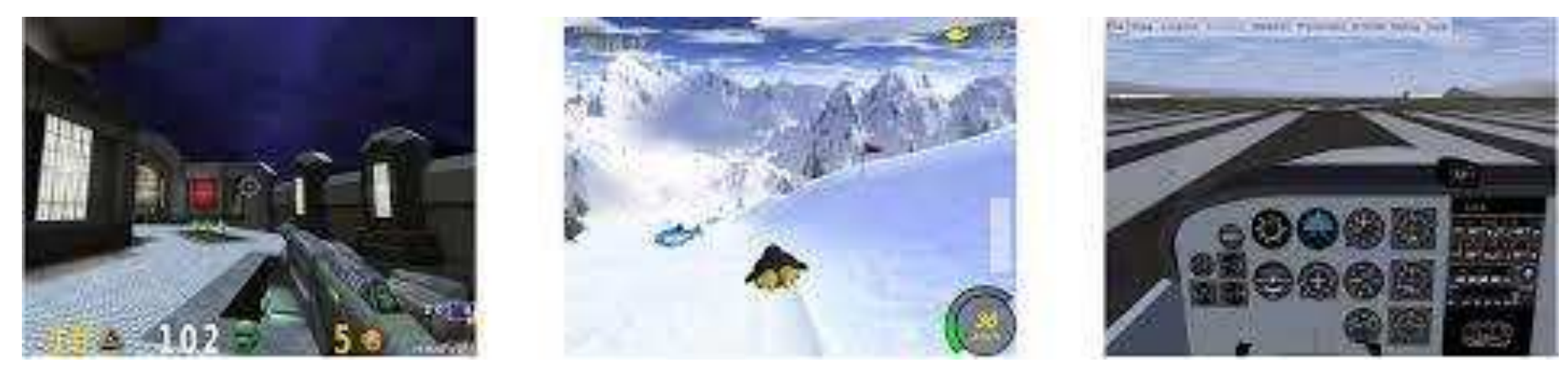

Intercept and stream OpenGL commands Better performances with respect to video streaming Limitation: clients need powerful GPU 


\section{Mixed Mobile/Remote rendering}

- Model based versus Image based methods

- Model based methods

- Original models

Eisert and Fechteler. Low delay streaming of computer graphics (ICIP 2008)

- Partial models

- Simplified models

Gobbetti et al. Adaptive Quad Patches: an Adaptive Regular Structure for Web Distribution and Adaptive Rendering of 3D Models. (Web3D 2012)

Balsa et al.,. Compression-domain Seamless Multiresolution Visualization of Gigantic Meshes on Mobile Devices (Web3D 2013)

- Couple

More details in Part 4

- Point d

mobile devices (IEEE Trans. on CG \& Appl, 2004) 


\section{Mixed Mobile/Remote rendering}

- Image based methods

- Image impostors

Noimark and Cohen-Or. Streaming scenes to mpeg-4 video-enabled devices (IEEE, CG\&A 2003)

Lamberti and Sanna. A streaming-based solution for remote visualization of 3D

graphics on mobile devices (IEEE, Trans. VCG, 2007)

- Environment maps

Bouquerche and Pazzi. Remote rendering and streaming of progressive panoramas for mobile devices (ACM Multimedia 2006)

- Depth images

Zhu et al. Towards peer-assisted rendering in networked virtual environments (ACM Multimedia 2011)

Shi et al. A Real-Time Remote Rendering System for Interactive Mobile Graphics (ACM Trans. On Multimedia, 2012)

Doellner et al. Server-based rendering of large 3D scenes for mobile devices using Gbuffer cube maps (ACM Web3D, 2012) 


\section{Mixed Mobile/Remote rendering}

- Image based methods

- Image impostors

Noimark and Cohen-Or. Streaming scenes to mpeg-4 video-enabled devices (IEEE, CG\&A 2003)

Lamberti and Sanna. A streaming-based solution for remote visualization of 3D graphic

- Envirc

Bouque Image representations are created by the server, for mok and warped in real time by the client to account for

- Depth user interaction

Zhu et al. Towards peer-assisted rendering in networked virtual environments (ACM Multimedia 2011)

Shi et al. A Real-Time Remote Rendering System for Interactive Mobile Graphics (ACM Trans. On Multimedia, 2012)

Doellner et al. Server-based rendering of large 3D scenes for mobile devices using Gbuffer cube maps (ACM Web3D, 2012) 


\section{Mixed Mobile/Remote rendering}

- Model based vs Image based methods

- Constraints: rendering quality, bandwidth, interactivity

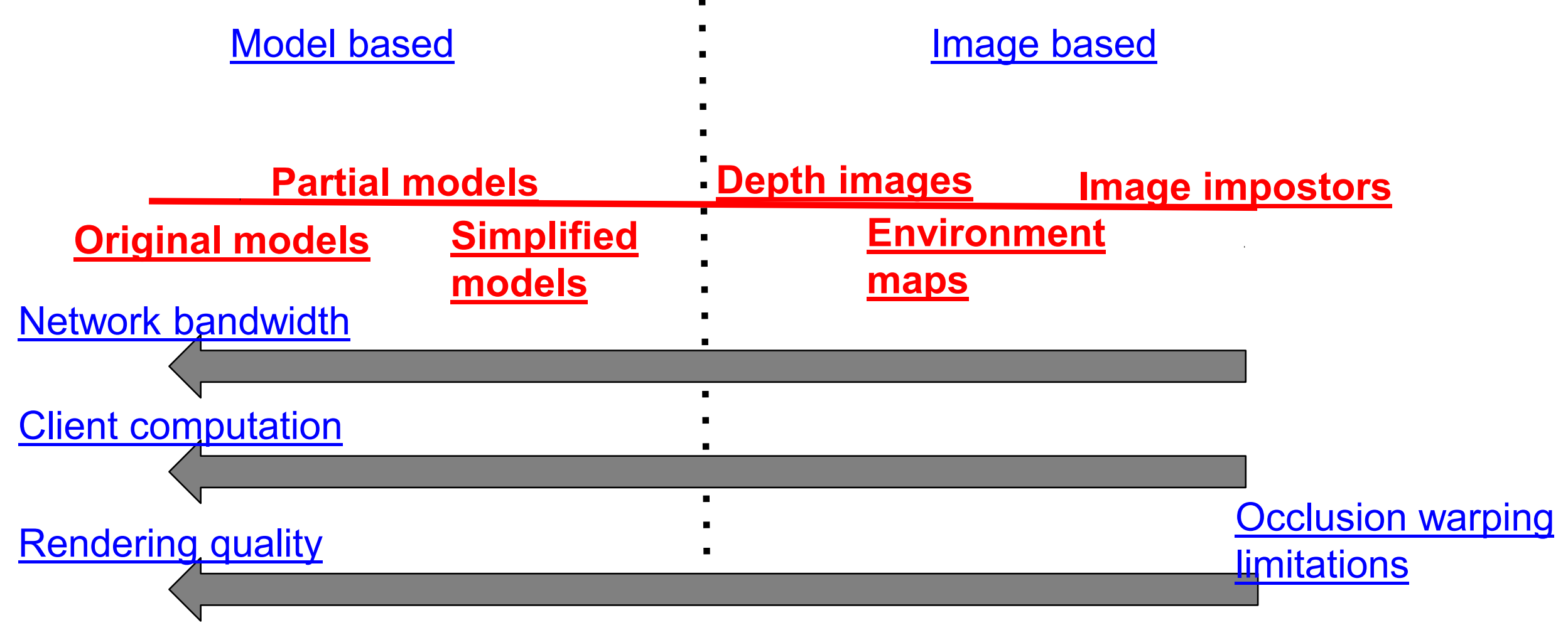




\section{Mobile visualization systems}

\section{- Volume rendering}

Moser and Weiskopf. Interactive volume rendering on mobile devices. Vision, Modeling, and Visualization VMV. Vol. 8. 2008.

Noguerat al. Volume Rendering Strategies on Mobile Devices. GRAPP/IVAPP. 2012.

Campoalegre, Brunet, and Navazo. Interactive visualization of medical volume models in mobile devices. Personal and ubiquitous computing 17.7 (2013): 1503-1514.

Rodríguez, Marcos Balsa, and Pere Pau Vázquez Alcocer. Practical Volume Rendering in Mobile Devices. Advances in Visual Computing. Springer, 2012. 708-718.

\section{- Point cloud rendering}

Balsa et al. Interactive exploration of gigantic point clouds on mobile devices. ( VAST 2012)

He et al. A multiresolution object space point-based rendering approach for mobile devices (AFRIGRAPH, 2007) 


\section{Mobile visualization systems}

\section{- Volume rendering}

Moser and Weiskopf. Interactive volume rendering on mobile devices. Vision, Modeling, and Visualization VMV. Vol. 8. 2008.

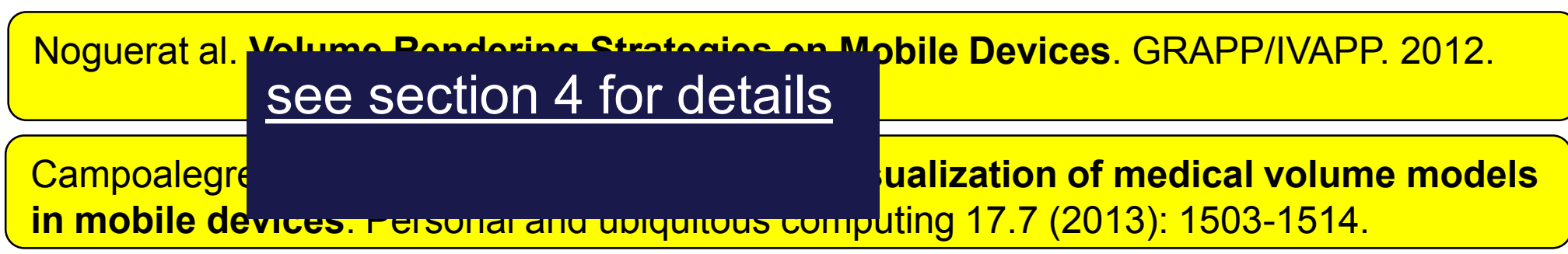

Rodríguez, Marcos Balsa, and Pere Pau Vázquez Alcocer. Practical Volume Rendering in Mobile Devices. Advances in Visual Computing. Springer, 2012. 708-718.

- Point cloud rendering

Balsa et al. Interactive exploration of gigantic point clouds on mobile devices. ( VAST 2012)

He et al. A multiresolution object space point-based rendering approach for mobile devices (AFRIGRAPH, 2007) 


\section{Mobile rendering}

- Nowadays...

\section{MOBILE DEVICE}

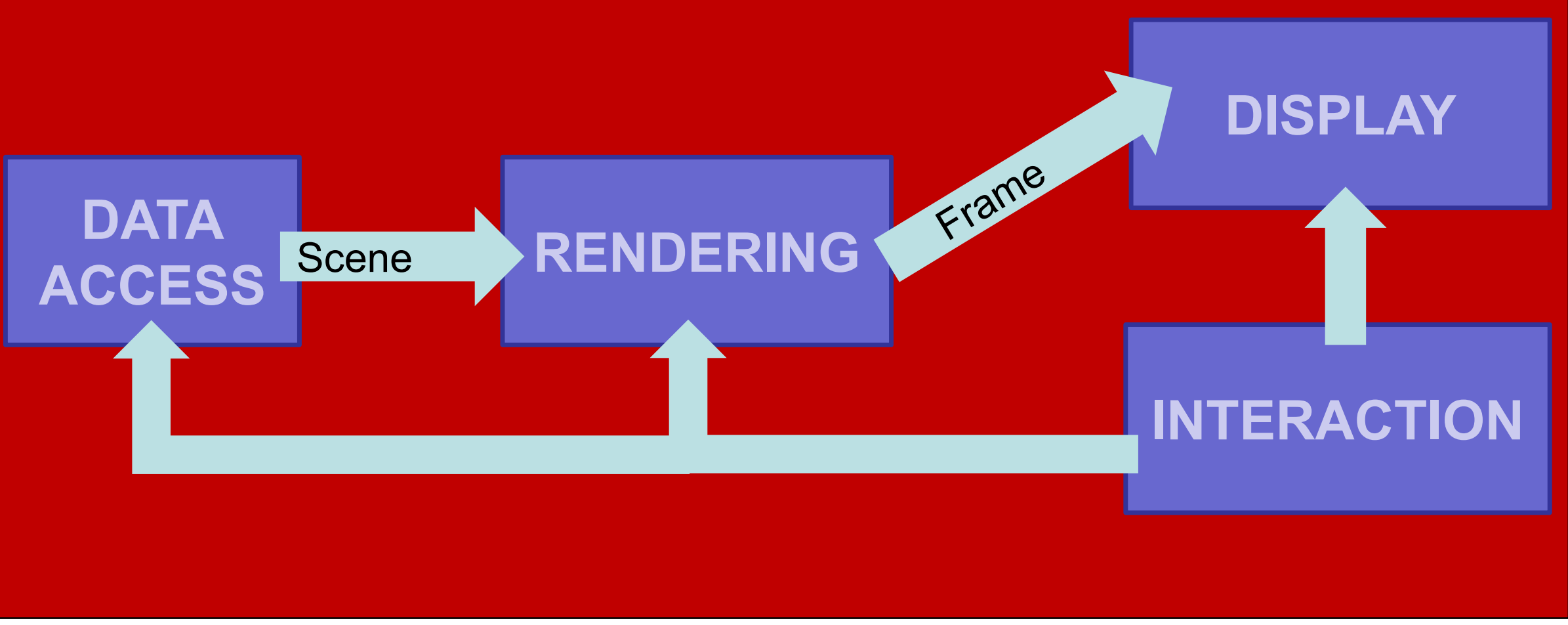




\section{Mobile rendering}

- Or better...

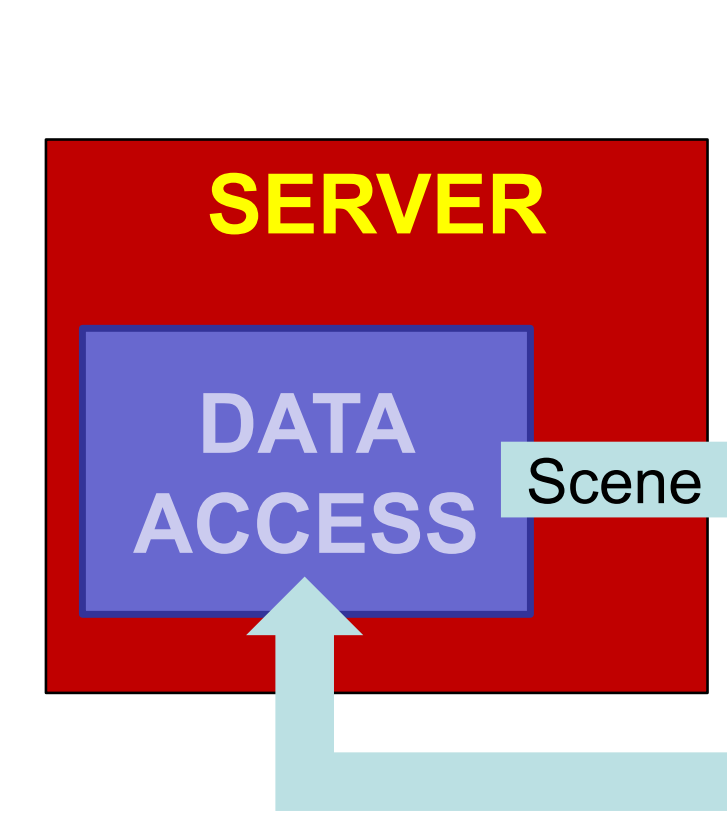

NETWORK:

\section{MOBILE DEVICE}

\section{DISPLAY}

RENDERING

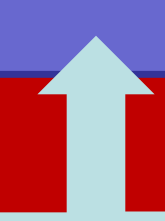

INTERACTION 


\section{Mobile rendering}

- Or better...

\section{SERVER}

Chunk-based data

D streaming

AC (like HuMoRS Balsa et

al. 2014)

Limitations: bandwidth consumption (for now)

NETWORK:

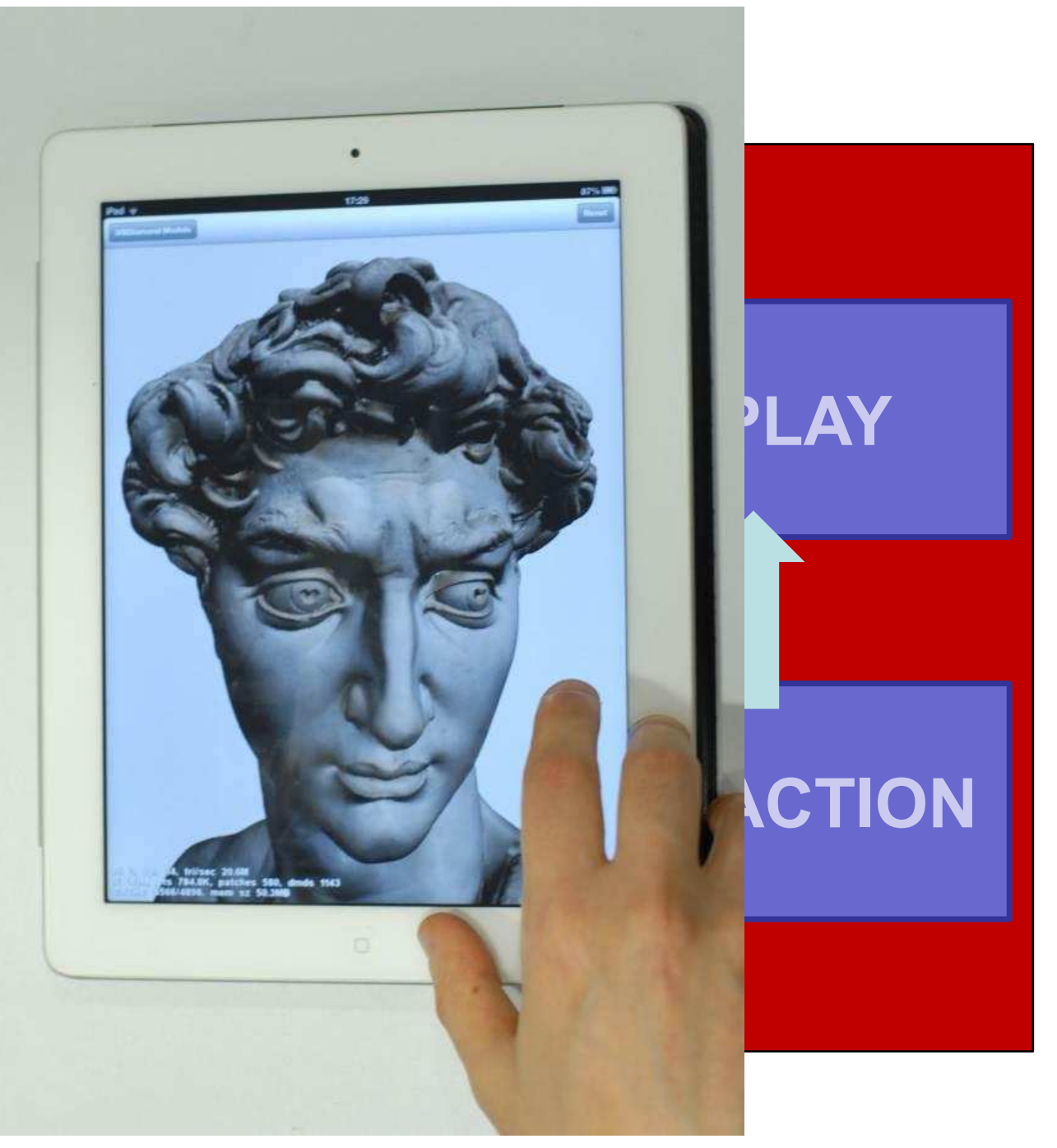




\section{Mobile rendering with capture}

- Exploiting mobile device sensors... MOBILE DEVICE

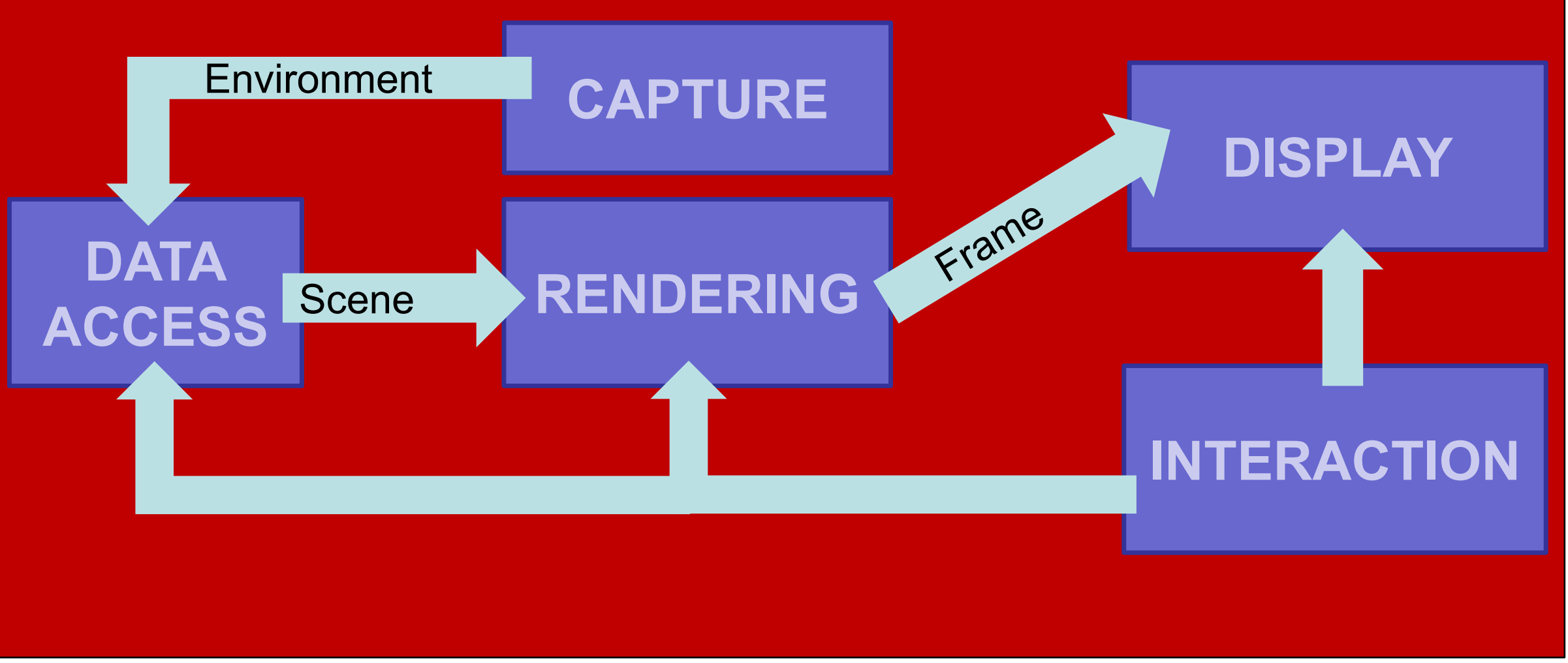




\section{Mobile rendering with capture}

- Exploiting mobile device sensors... MOBII F RFVIC.

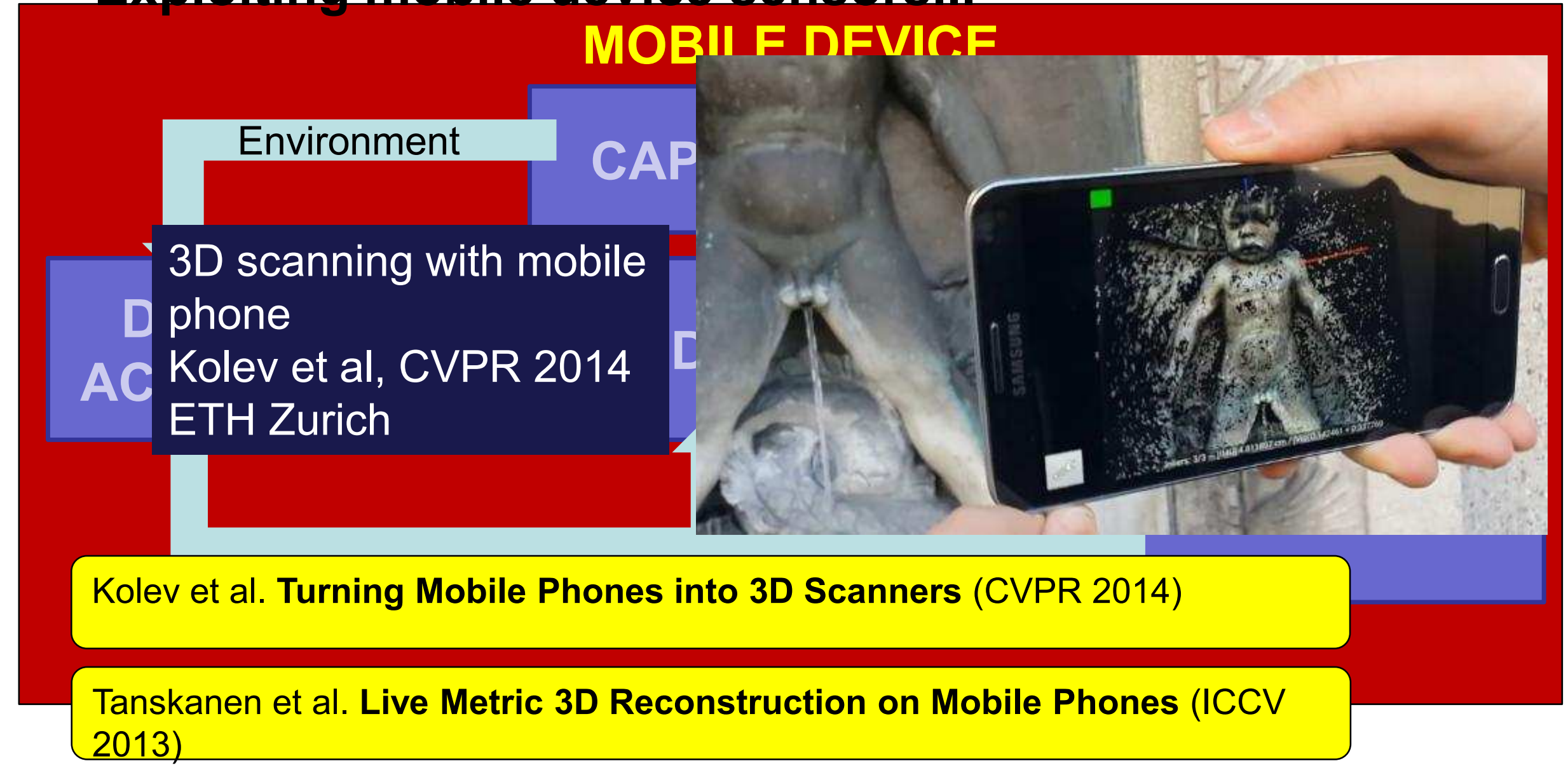
2013) 


\section{Mobile rendering with capture}

- Exploiting mobile device sensors... MOBILE DEVICE

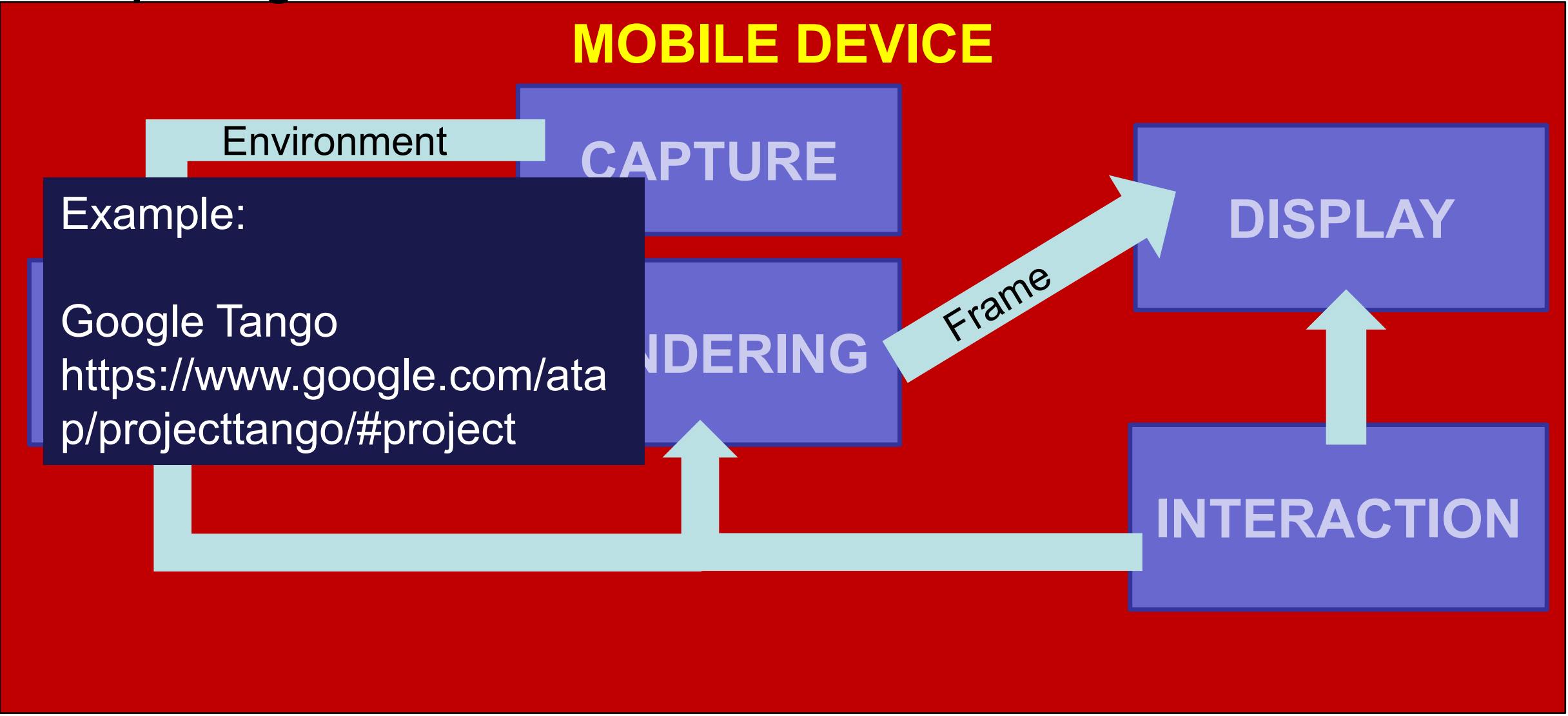




\section{Mobile rendering with capture}

- Exploiting mobile device sensors... MOBILE DEVICE

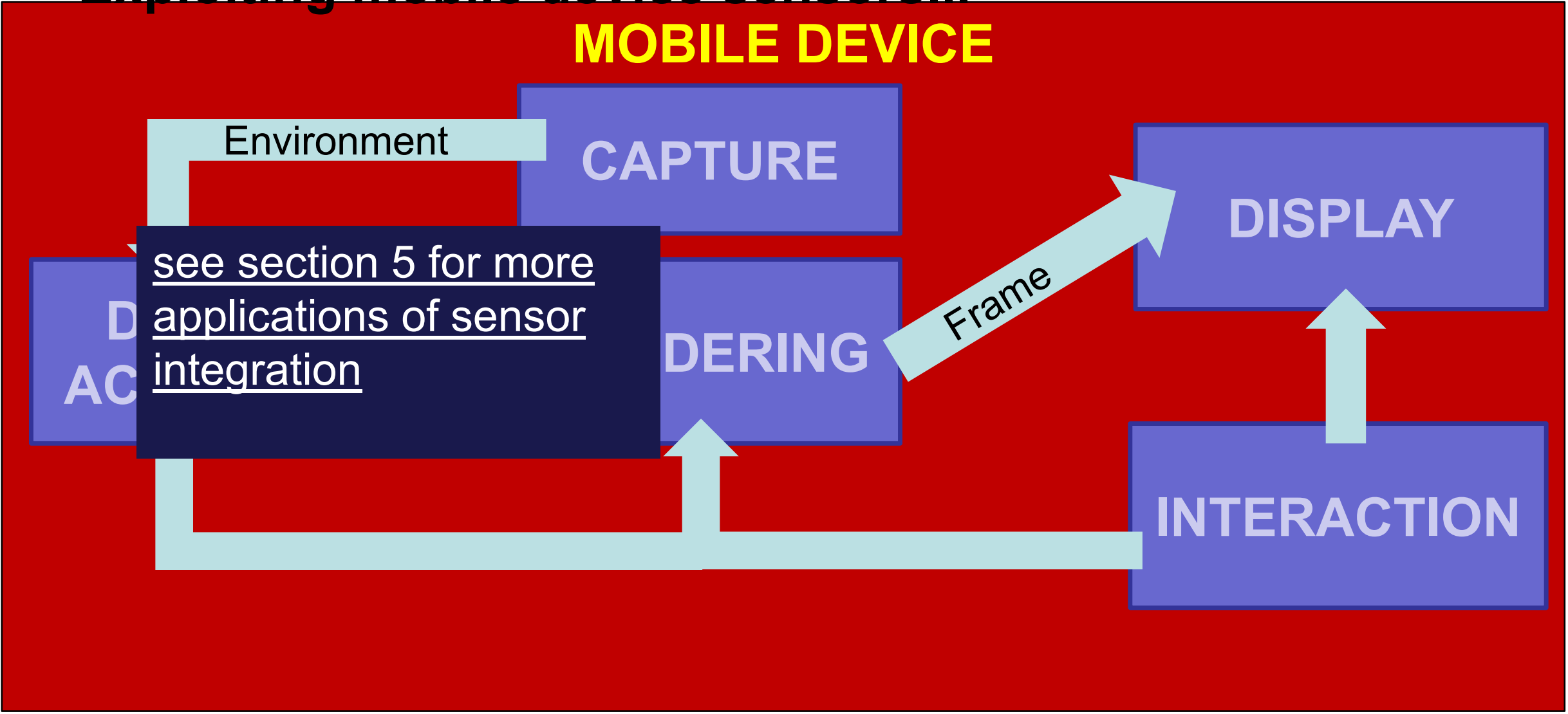




\section{Trends in mobile graphics}

- Hardware acceleration for improving frame rates, resolutions and rendering quality

- Parallel pipelines

- Real-time ray tracing

- Multi-rate approaches 


\section{SGRT: Real-time ray tracing}

\section{- Samsung reconfigurable GPU} based on Ray Tracing

- Main key features:

- an area-efficient parallel pipelined traversal unit

-flexible and high-performance kernels for shading and ray generation

Shin et al., Full-stream architecture for ray tracing with efficient data transmission, 2014 IEEE ISCAS

Lee, Won-Jong, et al. SGRT: A mobile GPU architecture for real-time ray

tracing. Proceedings of the 5th High-Performance Graphics Conference, 2013. 


\section{Adaptive shading}

- Triangles rasterized into coarse fragments that correspond to multiple pixels of coverage

- Coarse fragments are shaded, then partitioned into fine fragments for subsequent per-pixel shading
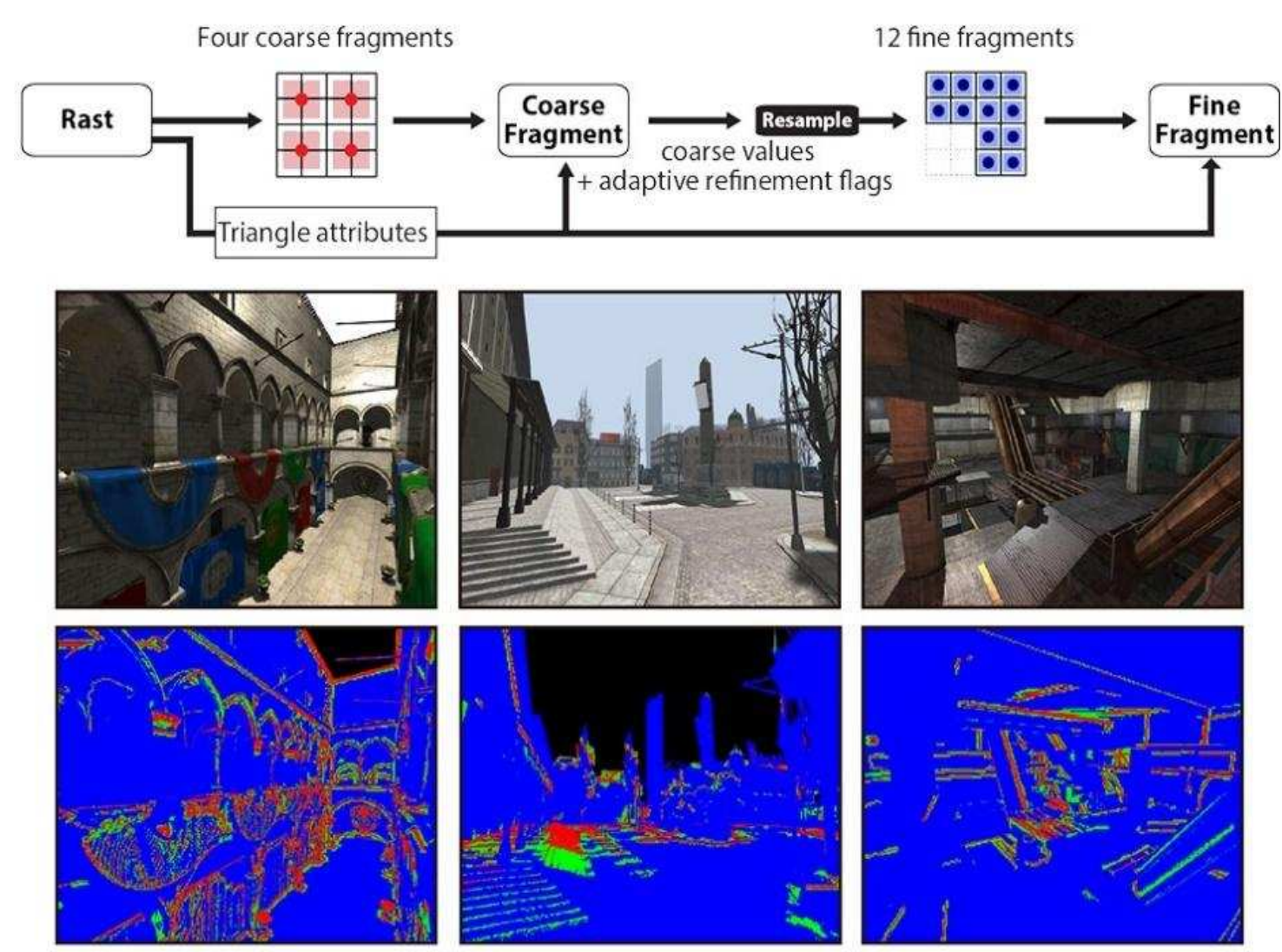

口 = fine (per-pixel) shading

$\square=\operatorname{COARSE} 2 \times 2$ shading

= COARSE $4 \times 4$ shading

He et al. Extending the graphics pipeline with adaptive, multi-rate shading. ACM Transactions on Graphics (TOG) 33.4 , 2014.

Clarberg, Petrik, et al. AMFS: adaptive multi-frequency shading for future graphics processors. ACM Transactions on Graphics (TOG) 33.4 , 2014.

Won-Jong Lee, et al. Adaptive multi-rate ray sampling on mobile ray tracing GPU. In SIGGRAPH ASIA 2016 Mobile Graphics and Interactive Applications (SA '16). 


\section{Mobile rendering with capture}

- Exploiting mobile device sensors... MOBILE DEVICE

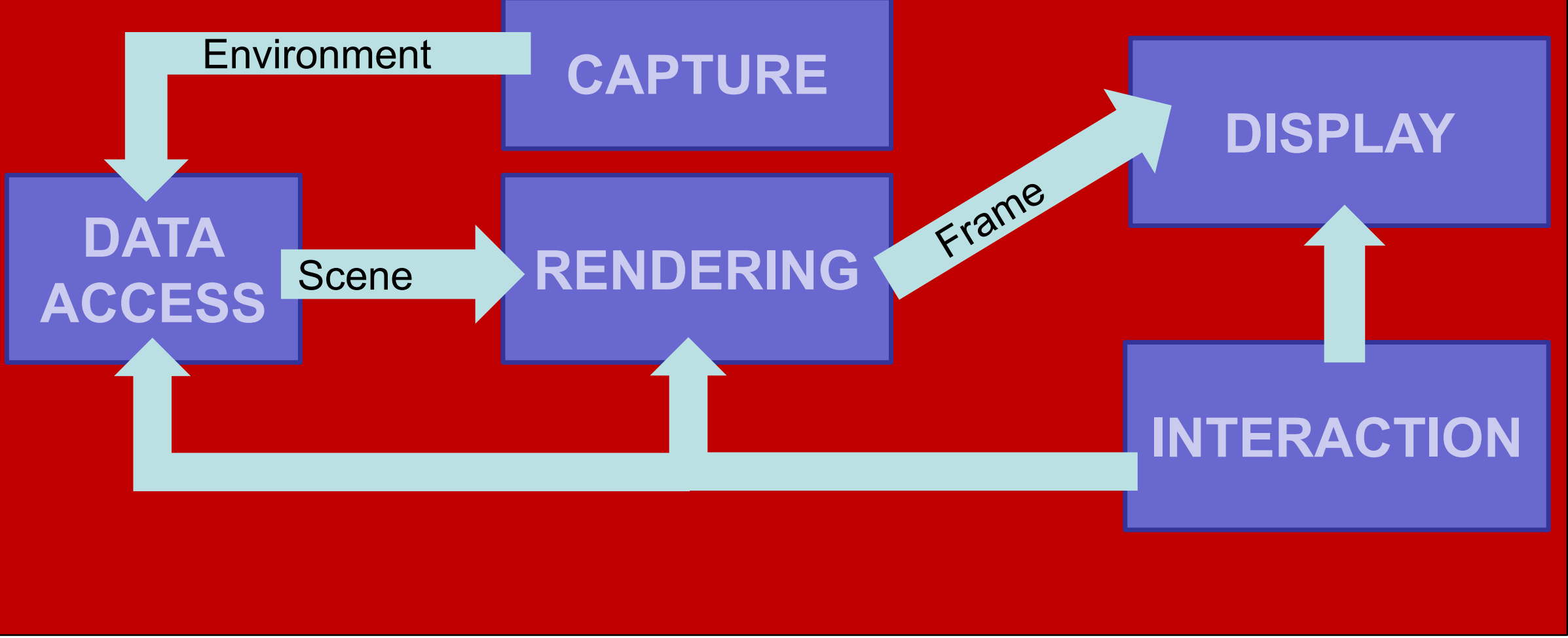




\section{Examples: Physical simulations}

- Framework for physically and chemically-based simulations of analog alternative photographic processes

- Efficient fluid simulation and manual process running on iPad

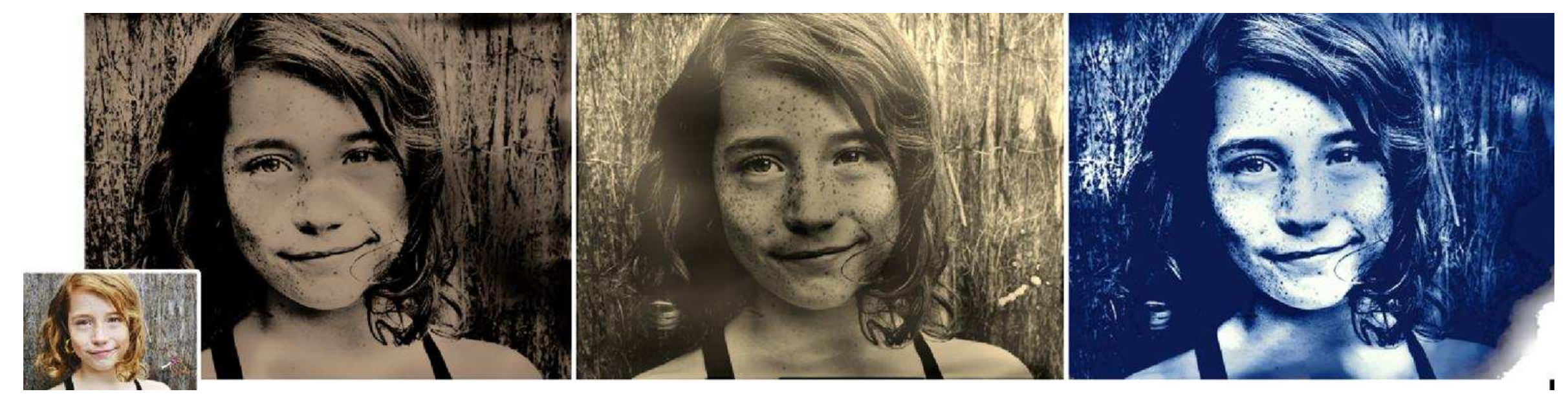

Echevarria et al. Computational simulation of alternative photographic processes. Computer Graphics Forum. Vol. 32. 2013. 


\section{Examples: Correcting visual aberrations}

- Computational display technology that predistorts the presented content for an observer, so that the target image is perceived without the need for eyewear

- Demonstrated in low-cost prototype mobile devices

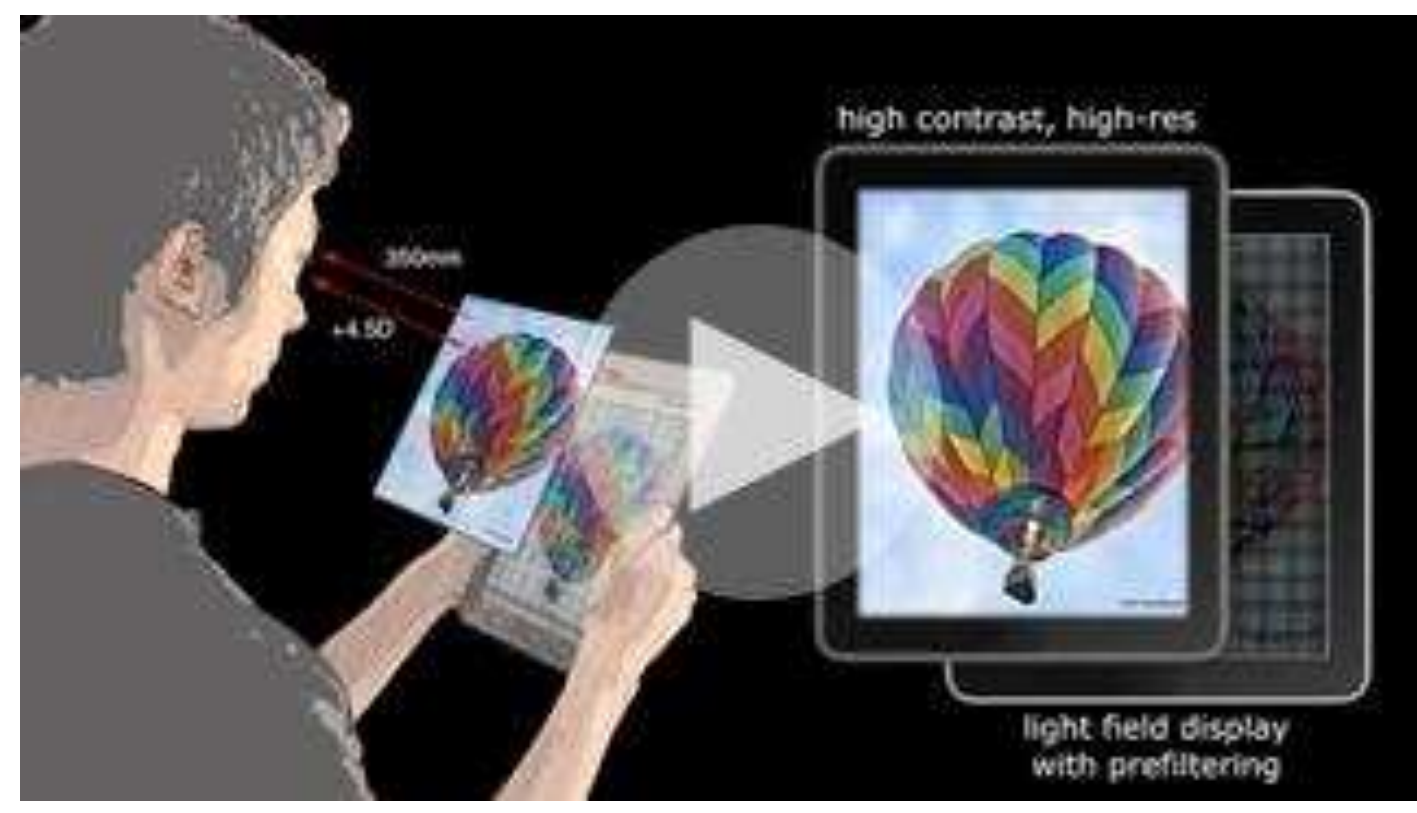

Huang, Fu-Chung, et al. Eyeglasses-free display: towards correcting visual aberrations with computational light field displays.ACM Transactions on Graphics (TOG) 33.4, 2014. 


\section{Conclusions}

- Heterogeneous applications

- driven by bandwidth and processing power

- Trends

- desktop software solutions tend to be ported to the mobile world

- gaming

- modelling and 3D animation

- complex illumination models

- Sensor integration open new scenarios

- examples: live acquisition, mHealth (using sensors and cameras for tracking and processing signals) 
Next Session

GRAPHICS DEVELOPMENT FOR MOBILE SYSTEMS 


\section{Part 3}

\section{Graphics development for mobile systems}

Marco Agus, KAUST \& CRS4 
Mobile Graphics

\section{Heterogeneity}

\section{os}

programming

languages

architecture

3D APIs

IDEs 
Mobile Graphics

\section{Heterogeneity}

\section{OS}

3D APIs 


\section{Mobile Graphics}

\section{OS}

\section{Programming Languages}

\section{Architectures}

3D APIs

\section{Cross-development}

Android

iOS

Windows Phone

Firefox OS, Ubuntu Phone, Tizen...

C++

Obj-C / Swift

Java

C\# / Silverlight

Html5/JS/CSS

X86 (x86_64): Intel / AMD

ARM (32/64bit): ARM + (Qualcomm, Samsung, Apple, NVIDIA,...)

MIPS (32/64 bit): Ingenics, Imagination.

OpenGL / GL ES

D3D / ANGLE

Metal / Mantle / Vulkan (GL Next)

Qt

Marmalade / Xamarin /

Muio

Monogame / Shiva3D / Unity / UDK4 / Cocos2d-x 


\section{Operating Systems}

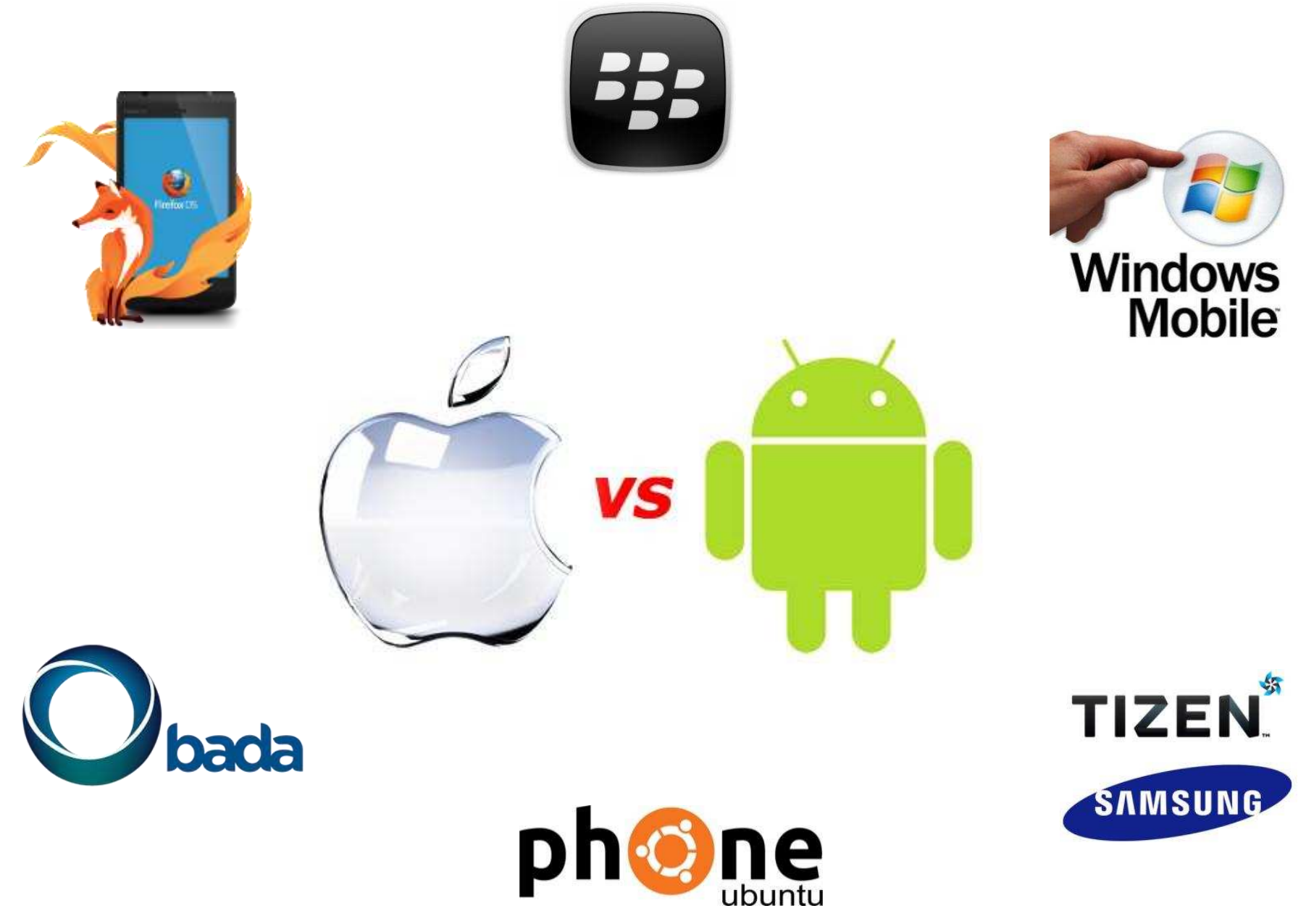




\section{Operating Systems}

- Linux based (Qt...)

- Ubuntu, Tizen, BBOS...

- Web based (Cloud OS)

- ChromeOS, FirefoxOS, WebOS

\section{- Windows Phone}

iOS ( unix + COCOA)

Android (JAVA VM)
Global smartphone sales to end users from 1st quarter 2009 to 3rd quarter 2016, by operating system (in million units)

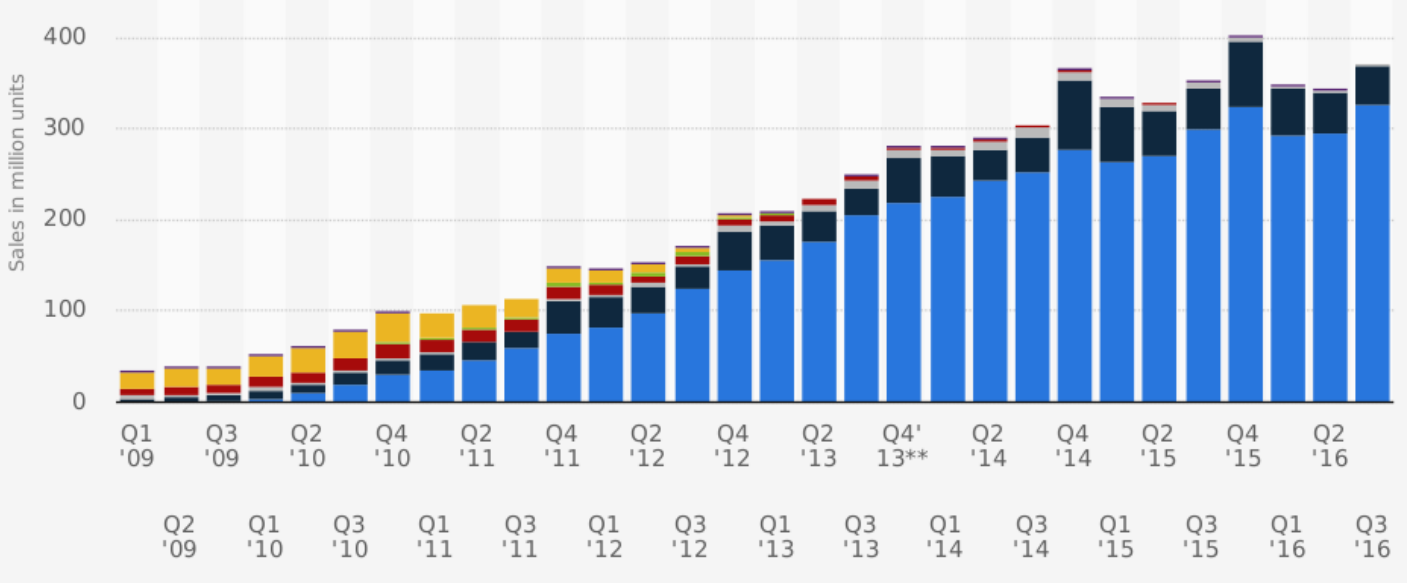

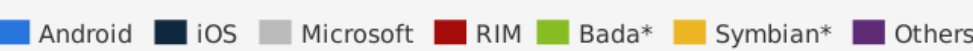




\section{Development trends}

- Hard to follow the trends

- software does not follow hardware evolution

- strong market oriented field where finance has strong impact on evolution

- In general, for

- Mobile phones

- Market drive towards Android, iOS

- Tablets

- Android, iOS, Windows 10

- Embedded devices

- Heterogenous (beyond the scopes of this course)

- Here we focus on mobile phones and tablets 


\section{Operating Systems}

\section{- Windows 10}

- Windows development - Visual Studio 2017

- Good debugging / compiler / integration

- Great integration and deployment

- Universal Windows Platform (UWP)

- API access

- C\#, VB.NET, and C++

$-3 \mathrm{D} A P I$

- D3D

- OpenGL access through ANGLE

- Advantages

- Visual Studio, interoperability with iOS

- HW is quite selected/homogeneous

- Disadvantages

- OpenGL wrapper just recently!

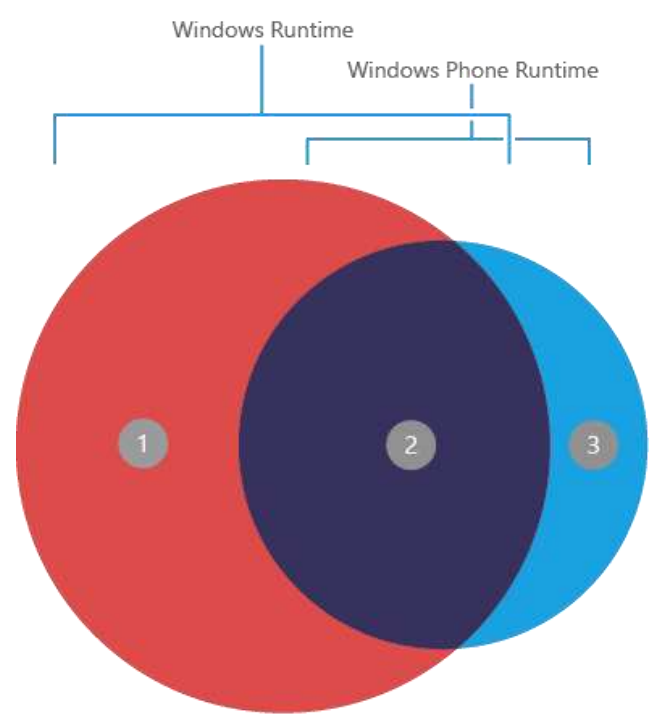

WINDOWS PHONE API

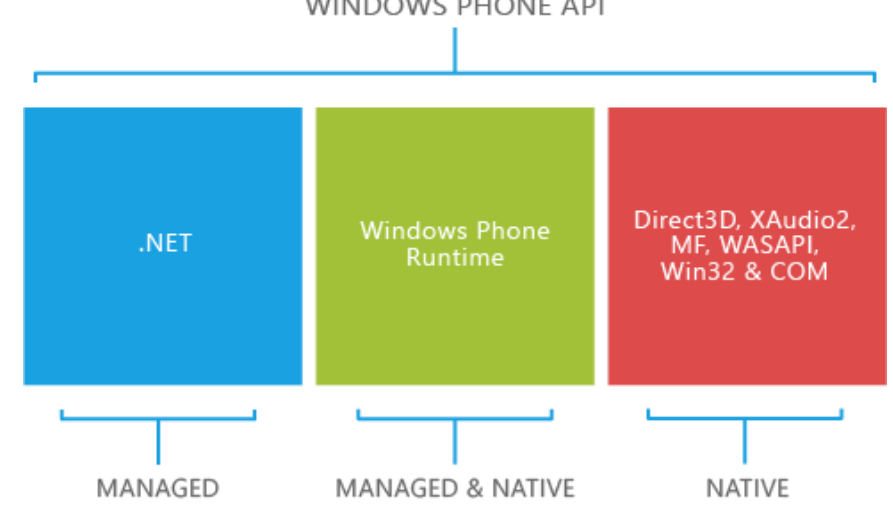




\section{Operating Systems}

- iOS

- Development under MacOS

- Xcode - good IDE/debug

- Clang compiler!

- API access

- Objective-C, swift

- Library programming

- C++ support

- Advantages:

- Homogeneous hardware (biggest issues are resolution related)

- State-of-the-art CPU/GPU (PowerVR SGX 54X/554, G6400)

- Good dev tools (Xcode + Clang)

- Inconvenients:

- Closed platform

- Requires iDevice for development/shipment (mostly) 


\section{Operating Systems}

\section{- Android}

- Development in Eclipse / AndroidStudio

- Java-based - integrated debugging (non-trivial for NDK)

- GCC / clang compilers

- Advantages

- Wide variety of hardware configurations (CPU/GPU)

- Java based + C++ as dynamic library (JNI or NDK+NativeActivity)

- Open source

- Toolchain provided for Windows/Linux/MacOS (GCC + Clang)

- Faster access to new hardware / functionality!

- Inconvenients

- Heterogeneous device base (hard to target all configurations)

- Not so integrated IDE -- mixed pieces 


\section{Operating Systems (comparison)}

\section{App development -- publishing}

- WinPhone \& iOS requires less effort for distribution

- Easy to reach the whole user base

- Android has a wide variety of configuration that require tuning

- User base is typically reached in an incremental way (supporting more configs)

- Many HW configurations (CPU/GPU) give more options to explore (-)

- Windows has not yet the same market share

- Variety of configurations 


\section{Programming Languages}

- $\mathbf{C} / \mathbf{C + +}$

- Classic, performance, codebase, control

Objective C

- Bit different style (message based), well-documented API for iOS, mainly COCOA/iOS

Java

- Android is VM/JIT based, portability (API), well-known, extended, codebase

C\#

- VM based, Java evolution, (Win, Android, iOS)

\section{Swift}

- Apple new language, simplicity, performance, easy, LLVM-based compilers

\section{HTML5/JS}

- Web technologies, extended, compatibility

- Perl, Python, Ruby, D, GO (Google), Hack (facebook), ...

- More options, not so popular? 


\section{D APIs}

\section{HTML}

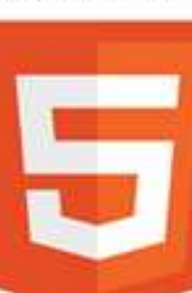

\section{OpenGL.}

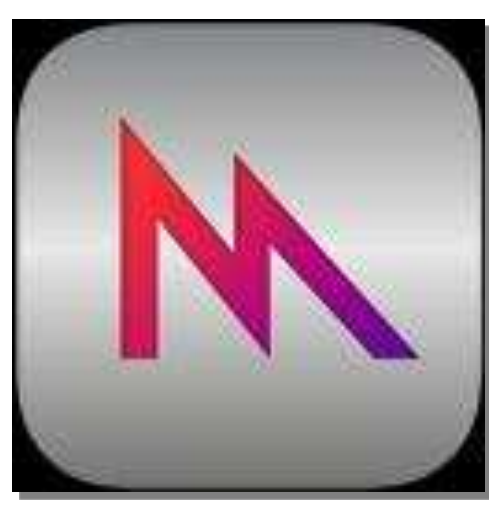

WebGL.

Vuliran EpenGLIES.
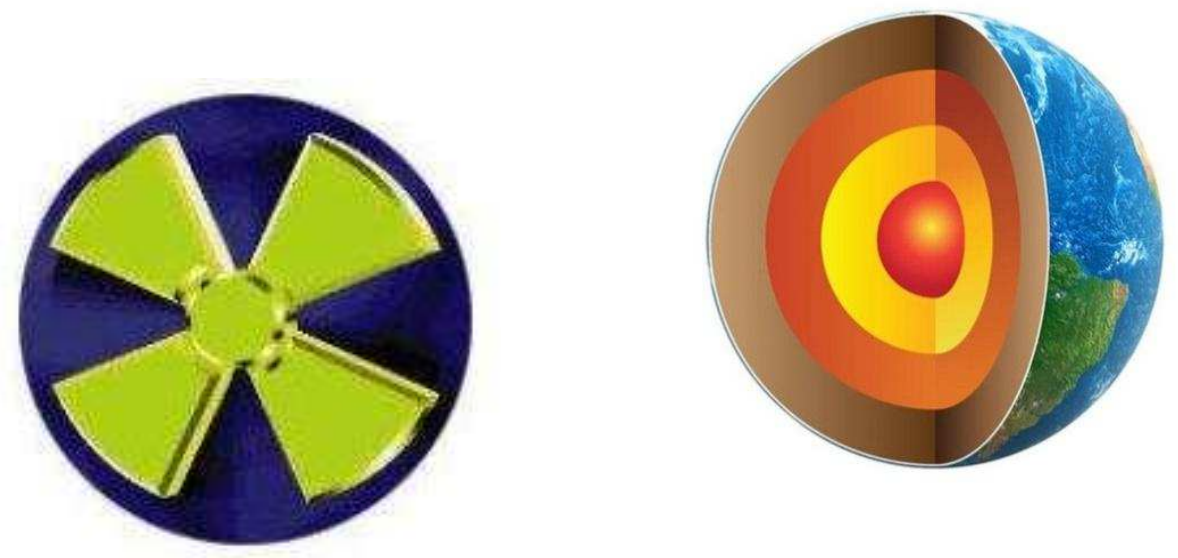


\section{D APIs}

\section{Cross Platform Challenge}

- An explicit API that is also cross-platform needs careful design
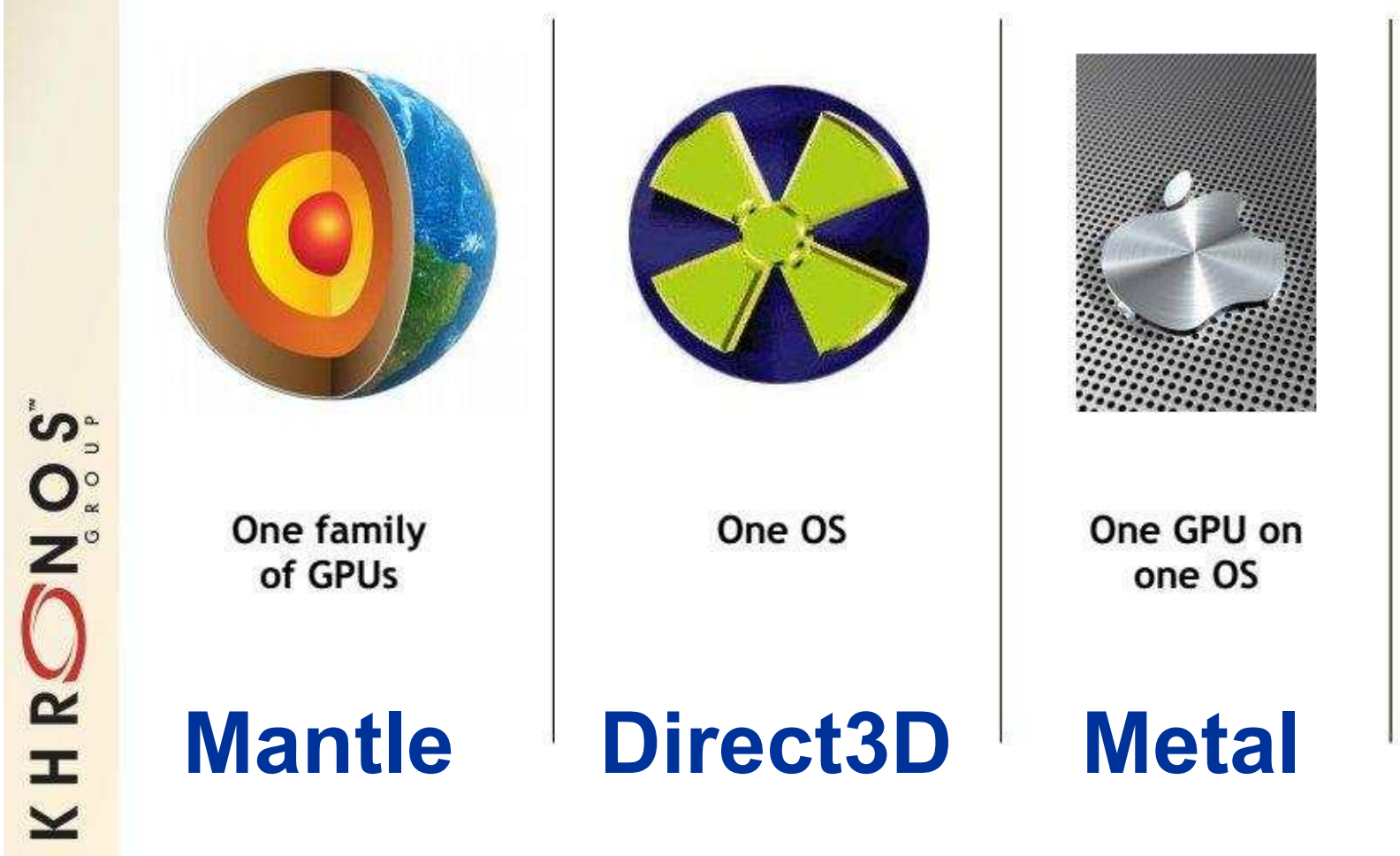

Vulikan.

OpenGL Next

5.0 


\section{D APls}

\section{- Direct 3D}

- 3D API from MS for Win OS (XBOX)

- ANGLE library provides GL support on top of D3D

\section{- Mantle}

- AMD 3D API with Low-level access $\rightarrow$ D3D12 | GL_NG

\section{- Metal}

- Apple 3D API with low-level access

\section{- OpenGL Desktop/ES/WebGL}

- GL for embedded systems, now in version 3.2

- GLES3.2 GL4.5

\section{GL Next Generation $\rightarrow$ Vulkan}

- redesign to unify OpenGL and OpenGL ES into one common API (no backward compatibility) 


\section{D APIs}

\section{- Direct 3D}

- Games on Windows (mostly) / XBOX

- Define 3D functionality state-of-the-art

- OpenGL typically following

- 3D graphic cards highly collaborative

- Multithread programming

- Proprietary - closed source - M\$

- Tested \& stable - good support + tools

\section{- Metal}

- Apple 3D API with low-level access

- Much in the way of Mantle?

- buffer \& image, command buffers, sync...

- Lean \& mean $\rightarrow$ simple $+\sim$ flexible
Win \&

Game research

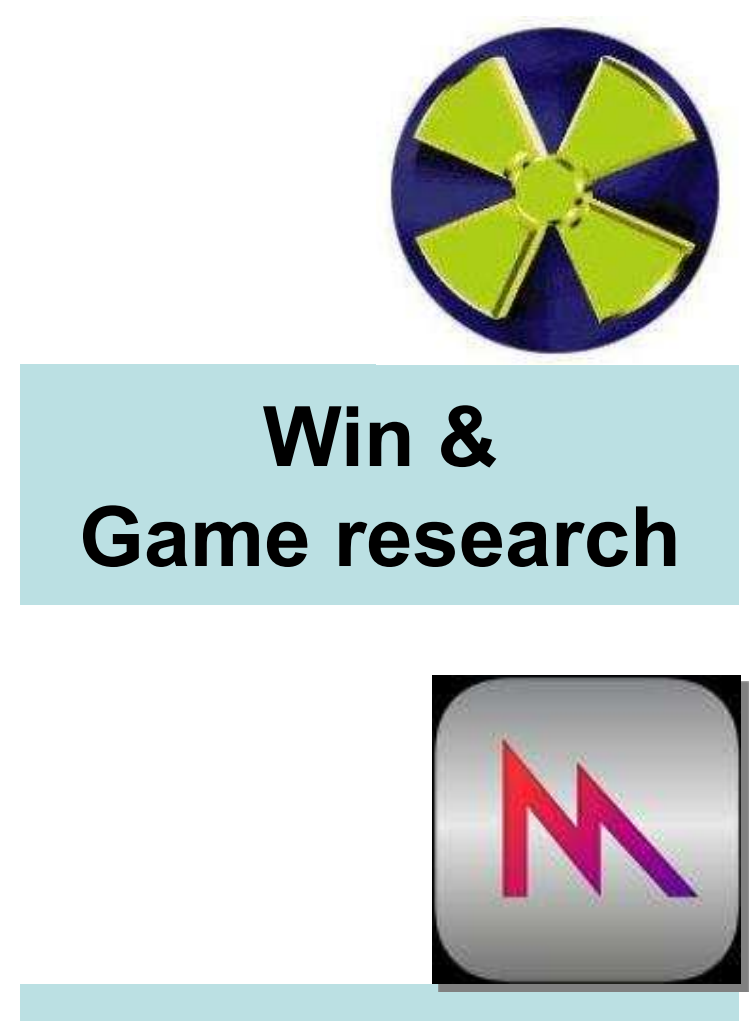

Mac/iOS future? 


\section{D APIs}

\section{Mantle}

- AMD effort - low level - direct access - 3D API

- Direct control of memory (CPU/GPU) - multithreading done well

- User-required synchronization

- API calls per frame $<3 k \rightarrow 100 K$

- Resources: buffer \& image $\odot$

- Simplified driver $\rightarrow$ maintenance (vendors)

- High level API/Framework/Engines will be developed $(-)$

- Pipeline state

- shaders + targets $($ depth/color...) + resources + geometry

- Command queues + synchronization

- Compute / Draw / DMA(mem. Copy)

- Bindless - shaders can refer to state resources

- OpenGL NEXT seems to move into 'Mantle direction'

- Direct 3D 12 already pursuing low-level access 


\section{D APIs}

\section{- OpenGL (Desktop/ES/WebGL)}

- Open / research / cross-platform

- Lagging in front of D3D $\rightarrow$ Legacy support $\odot$

- No more FIXED PIPELINE (1992)!! -- scientific visualization...

- GLSL (2003)...GL 3.1(2009) $\rightarrow$ deprecation/no fixed pipeline

- Compatibility profile $\rightarrow$ legacy again...(till GL 4 )

- Core profile

- GLSL $\rightarrow$ shader required

- VAO

" group of VBO

" we need a base VAO for using VBO!

- Simplifying $\rightarrow$ VBO + GLSL only! 


\section{D APIs}

- OpenGL ES 1.1

- Fixed pipeline - no glBegin/End - no GL_POLYGON -- VBO

- OpenGL ES 2 (OpenGL 1.5 + GLSL) GL4.1

- No fixed pipeline (shaders mandatory), ETC1 texture compress..

- OpenGL ES 3 GL4.3

- Occlusion queries + geometry instancing

- 32bit integer/float in GLSL

- Core 3D textures, depth textures, ETC2/EAC, many formats...

- Uniform Buffer Objects (packed shader parameters)

- OpenGL ES 3.2 GL4.5

- Compute shaders (atomics, load/store)

- Separate shader objects (reuse)

- Indirect draw (shader culling...)

- NO geometry/tessellation 


\section{D APIs}

\section{- Vulkan}

- derived from and built upon components of AMD's Mantle API

- with respect to OpenGL

- lower level API, more balanced CPU/GPU usage, parallel tasking, work distribution across multiple CPU cores

\begin{tabular}{|l|l|}
\hline OpenGL & Vulkan \\
\hline Global state machine & No global state \\
\hline State tied to context & Comman buffer instead of state \\
\hline Sequential operations & Multithreaded programming \\
\hline $\begin{array}{l}\text { Limited control of GPU memory } \\
\text { and sync }\end{array}$ & $\begin{array}{l}\text { Explicit control of memory man. } \\
\text { and sync }\end{array}$ \\
\hline Extensive error checking & No error checking at runtime \\
\hline
\end{tabular}




\section{D APIs}

\section{- GPGPU}

- OpenCL

- On Android it is not much loved

- Use GPU vendor SDK provided libs $\odot$

- On iOS is only accepted for system apps

- Use old-school GPGPU (fragment shader -> FrameBuffer)

- Compute shaders

- GLES 3.2!!! General solution!!

- DirectCompute on D3D 


\section{Cross-development}

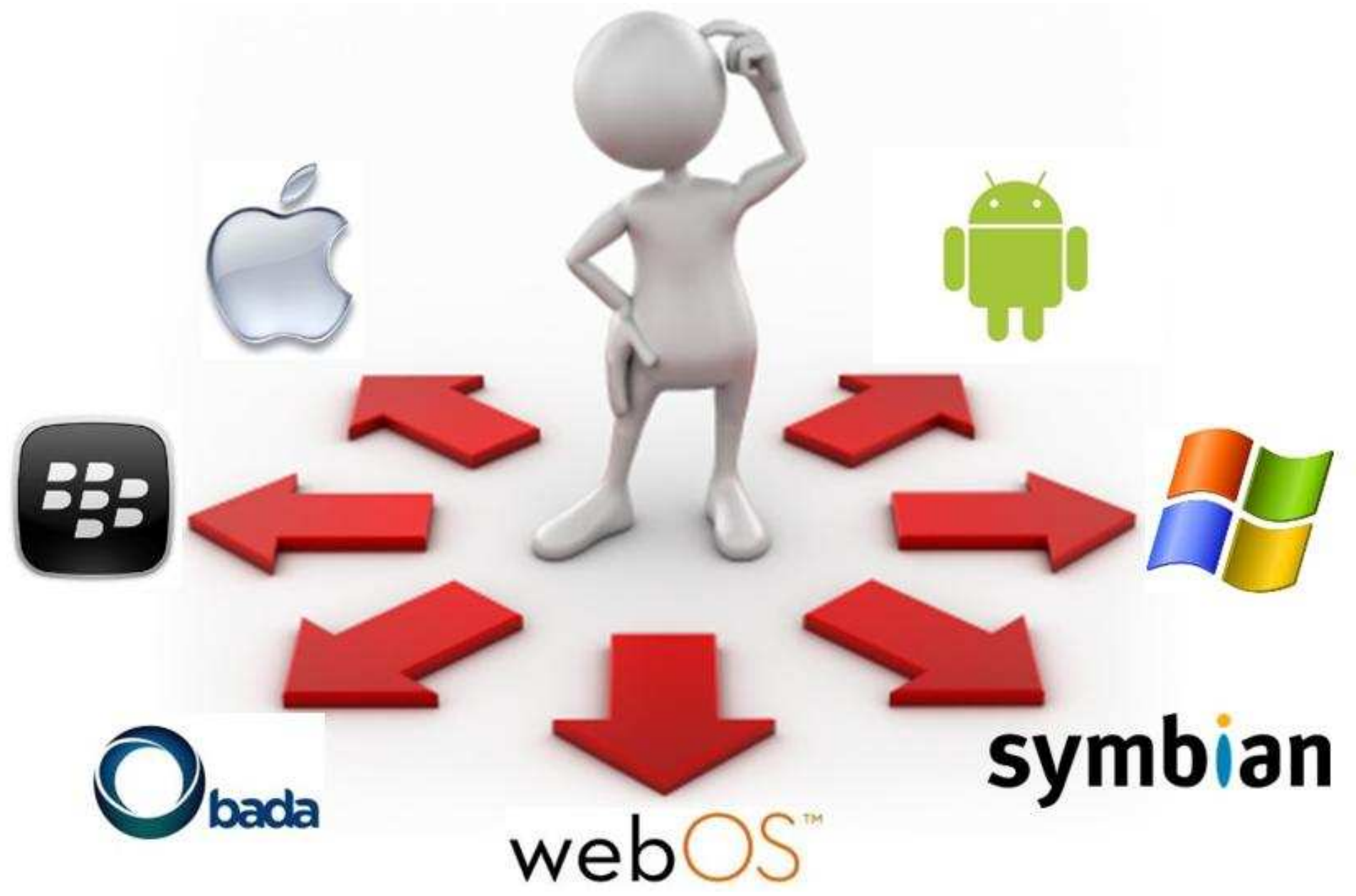

http://www.appian.com/blog/enterprise-mobility-2/are-mobile-platform-choices-limiting-enterprise-process-innovation 


\section{Cross platform}

- Unity Mobile (for gaming and VR)

- iOS/Android, integration with Tango

- Unreal Engine 4 (for gaming and VR)

- iOS/Android

- former Unreal Development Kit

- free usage, payment only for shipping

- Corona SDK

- iOS /Android

- uses integrated Lua layered on top of $\mathrm{C}++/$ OpenGL to build graphic application

- audio and graphics, cryptography, networking, device information and user input 


\section{Cross platform}

\section{- Marmalade}

- iOS/Android/Windows

- two main layers

- low level C API for memory management, file access, timers, networking, input methods (e.g. accelerometer, keyboard, touch screen) and sound and video output.

- C++ API for higher level functionality for 2D (e.g. bitmap handling, fonts) 3D graphics rendering (e.g. 3D mesh rendering, boned animation), resource management system and HTTP networking.

- Very successful but dismissing by March 2017

\section{- EdgeLib}

- iOS/Android/Windows

- high performance graphics engine in $\mathrm{C}++$

- support for 2D graphics, 3D graphics (OpenGL ES), input and sound 


\section{Cross platform}

\section{- JMonkey Engine}

- Android

- written in Java and using shader technology extensively

- uses LWJGL as its default renderer (another renderer based on JOGL is available, supporting OpenGL 4)

\section{- PowerVR}

- iOS/Android/Windows

- a cross-platform OS and API abstraction layer, a library of helper tools for maths and resource loading

- optimized for PowerVR GPUs, with Vulkan support

\section{- ARM Developer Center}

- Plenty of tools (computer vision and machine learning, OpenGL ES emulator, texture compression) 


\section{Cross-development}

\section{- C++ use case: QtCreator}

- Qt ( supports android, iOS, windows phone, linux, windows, mac)

- Provides API abstraction for UI, in-app purchases, touch input

- HOWTO (i.e. android):

- Android SDK

- Android NDK (native C++ support, toolchain, libraries, GL, CL...)

- Point environment variables ANDROID_SDK, ANDROID_NDK to folders

- Create new android project

- Play!

- Notes:

- Go for Qt > 5.4 (touch events were tricky in previous versions)

- Use QOpenGLWidget instead of QGLWidget

- Enable touch events on each widget:

- QWidget::setAttribute(Qt::WA_AcceptTouchEvents); 


\section{Mobile Graphics - Development}

\section{- Conclusions}

- 1) Native + platform UI ...

- $\mathrm{C}++$ [any language] $\rightarrow$ LLVM compiler $\rightarrow$ target platform

- Platform Framework front-end $\rightarrow \mathbf{1}$ for each platform

- Performance + flexibility

- Call native code from platform code $(\mathrm{JNI}$, Object C, ...)

- 2) Native through framework ...

- Qt | Marmalade ..

- $\mathrm{C}++$ code uses framework API

- Framework API abstracts platform API [N platforms]

- BUT less flexible integration?

- 3) Go web $\rightarrow$ HTML5/JS ...

- JS code + WebGL

- Free portability (chrome / firefox / IE ... ?)

- BUT performance is $0.5 \mathrm{X}$ at most with asm.js 
Next Session SCALABLE MOBILE VISUALIZATION 


\section{Part 4.1}

\section{Scalable Mobile Visualization: Introduction}

Enrico Gobbetti, CRS4 


\section{Scalable mobile visualization}

- Goal is high quality interactive rendering of complex scenes...

- Large data, shading, complex illumation,

- ... on mobile platforms ...

- Mostly smartphones or tablets

- Similar considerations can apply to other settings (e.g., embedded systems)
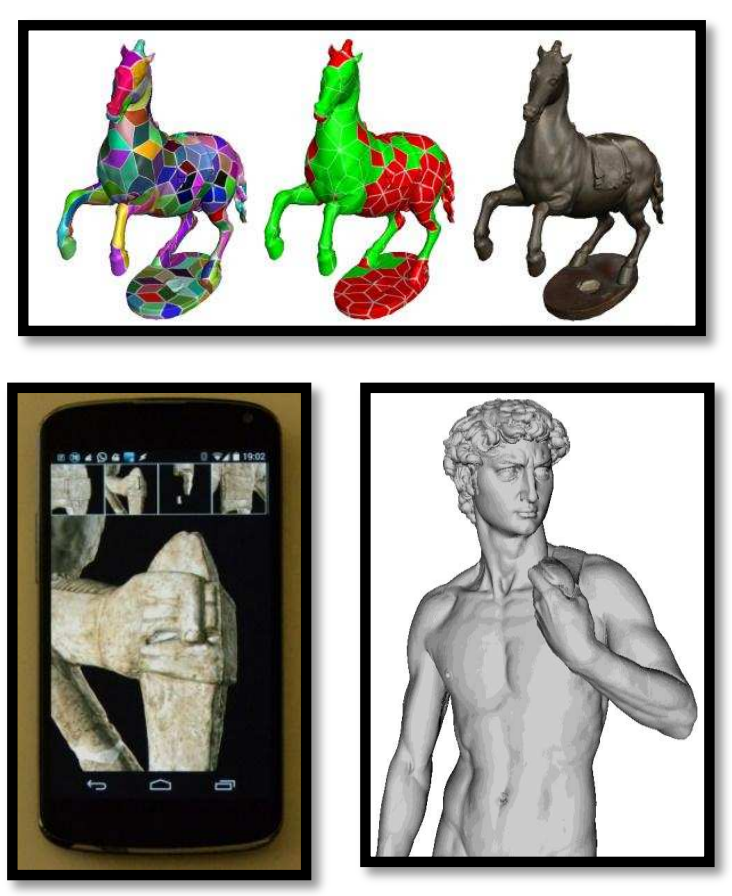

- Wide variety of applications

- Gaming, visualization, cultural heritage...
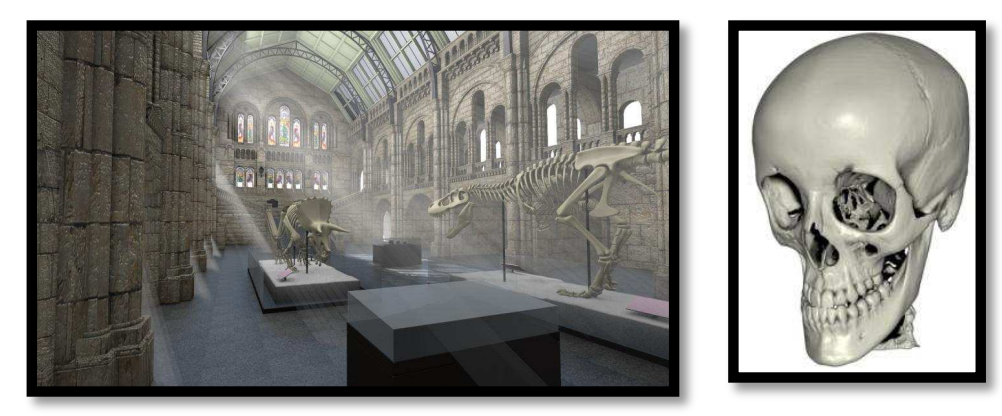


\section{Mobile platforms scenario}

- Typical scalable rendering problem, but with some specific constraints wrt standard (desktop settings)

- ... screen resolutions are often extremely large (2 - 6 Mpix)

- Lots of pixels to generate!

- ... mobile 3D graphics hardware is powerful but still constrained

- Reduced computing powers, memory bandwidths, and amounts of memory wrt

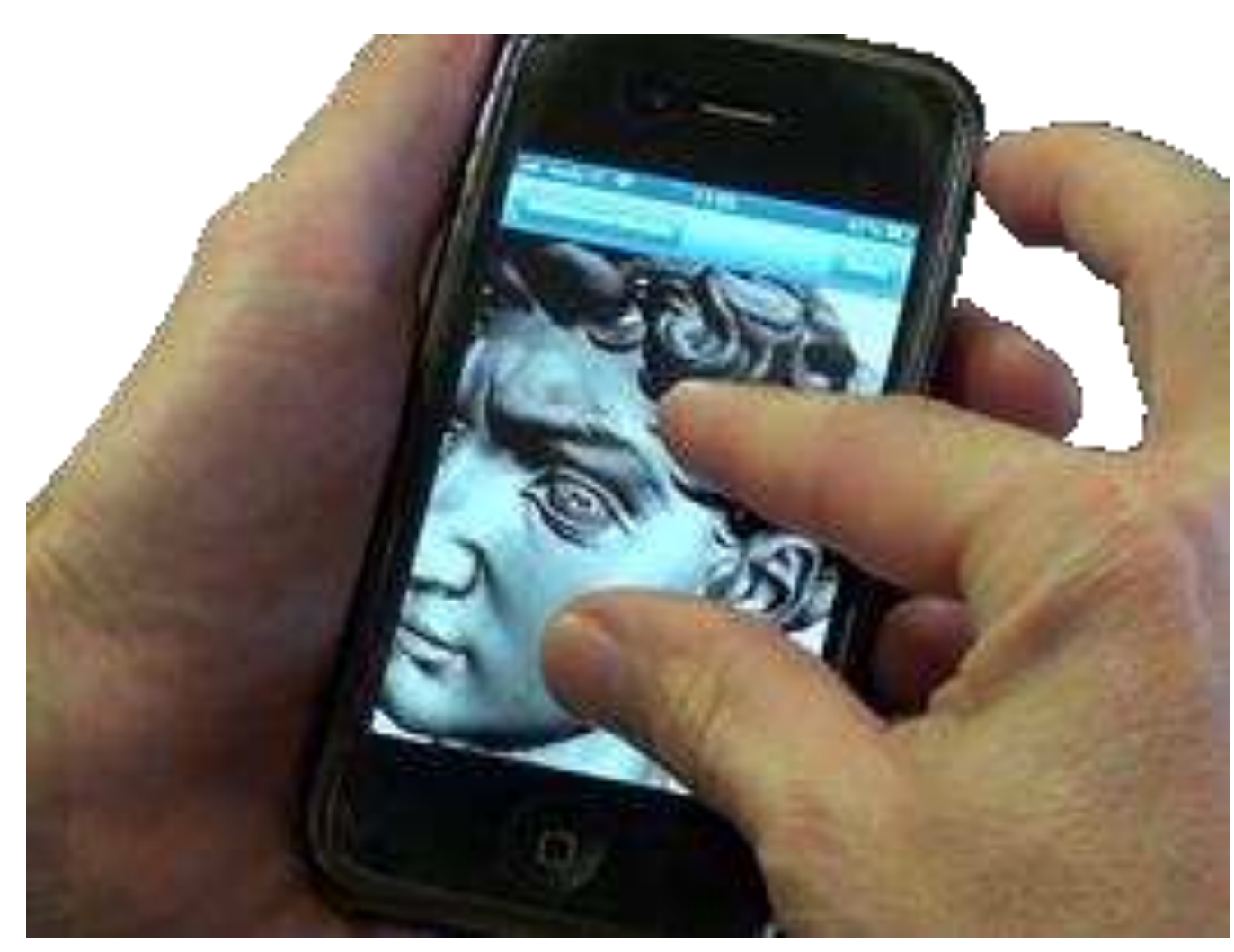
desktop graphics systems

- Limited power supply! 


\section{Mobile rendering scenario}

- No brute force method applicable

- Need for "smart methods" to perform interactive rendering

- Exploit at best reduced rendering power

\section{- Proposed solutions}

- Render only necessary data: adaptive multiresolution

- Limit required CPU/GPU work: full or partial precomputation

- Limit data requirements: streaming approaches

- Exploit at best available bandwidth: data compression

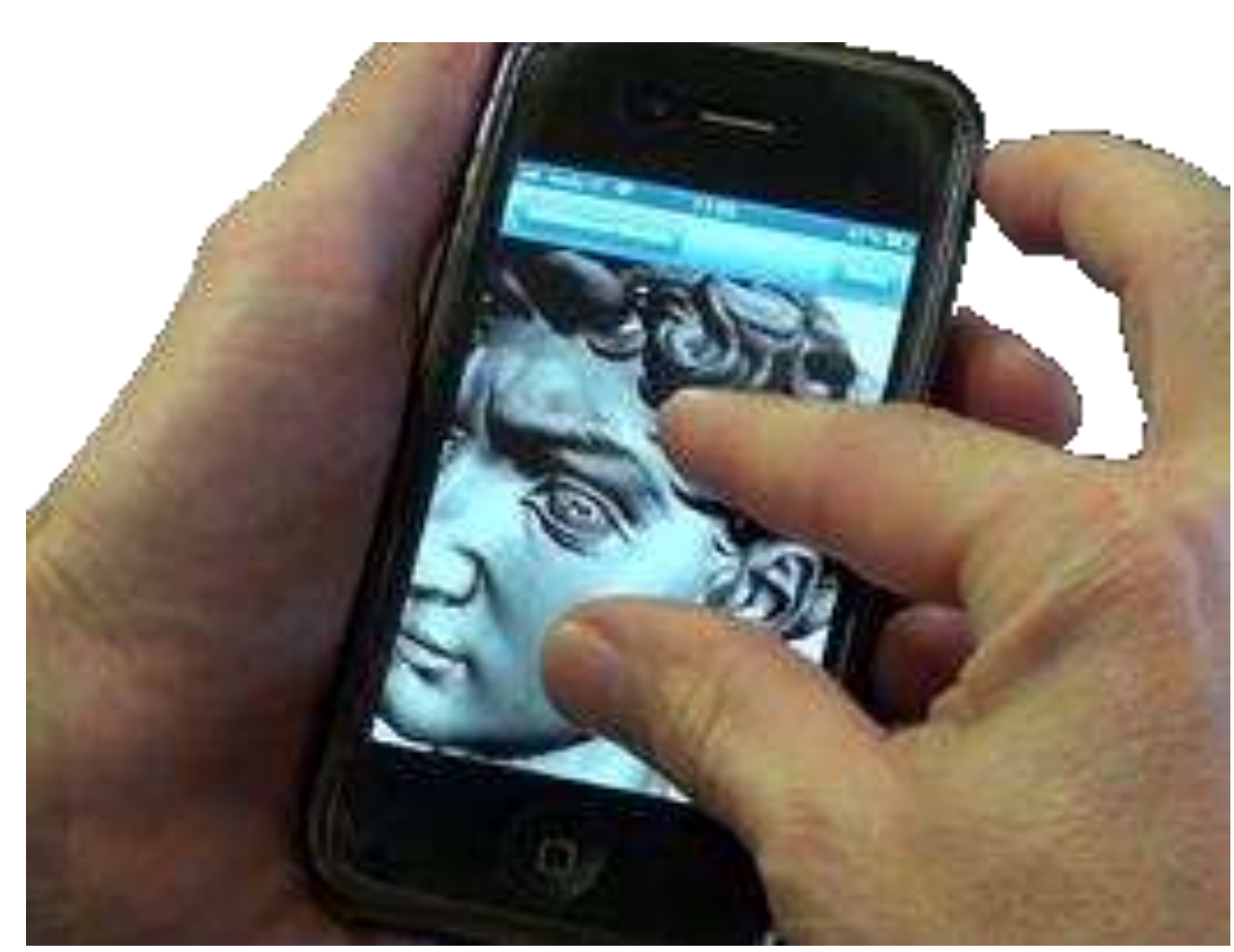




\section{Related Work on mobile visualization}

- (See previous session for details)

- Remote Rendering

$-\ldots . .$.

- Local Rendering

- Model based

- Original models

- Multiresolution models

- Simplified models

- Line rendering

- Point cloud rendering

- Image based

- Image impostors

- Environment maps

- Depth images

- Smart shading

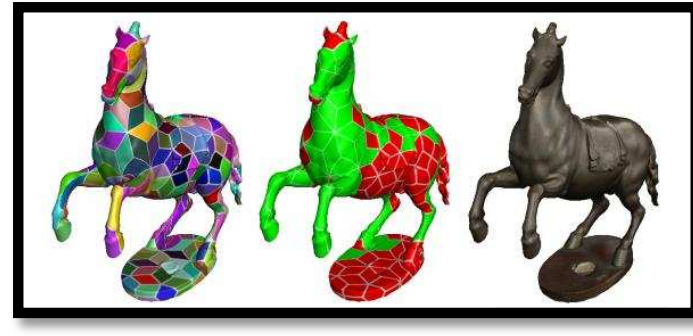

- Volume rendering 


\section{Related Work on mobile visualization}

- (See previous session for details)

- Remote Rendering

$-\ldots . .$.

- Local Rendering

- Model based

- Original models

- Multiresolution models

- Simplified models

- Line rendering

- Point cloud rendering

- Image based

- Image impostors

- Environment maps

- Depth images

- Smart shading

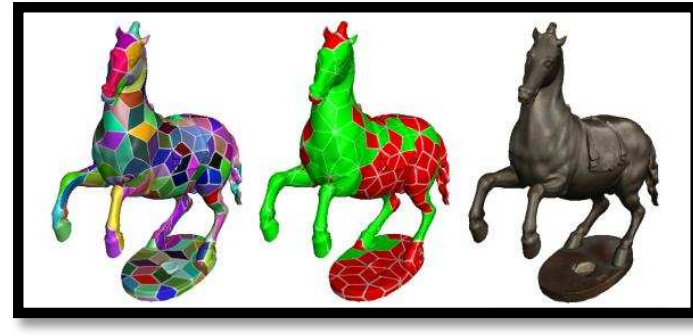

- Volume rendering 


\section{Scalable Mobile Visualization}

- Big/complex models:

- Detailed scenes from modeling, capturing..

- Output sensitive: adaptive multiresolution

- Compression / simple decoding

\section{- Complex rendering}

- Global illumination

- Pre-computation

- Smart shading

- Volume rendering

- Compression / simple decoding
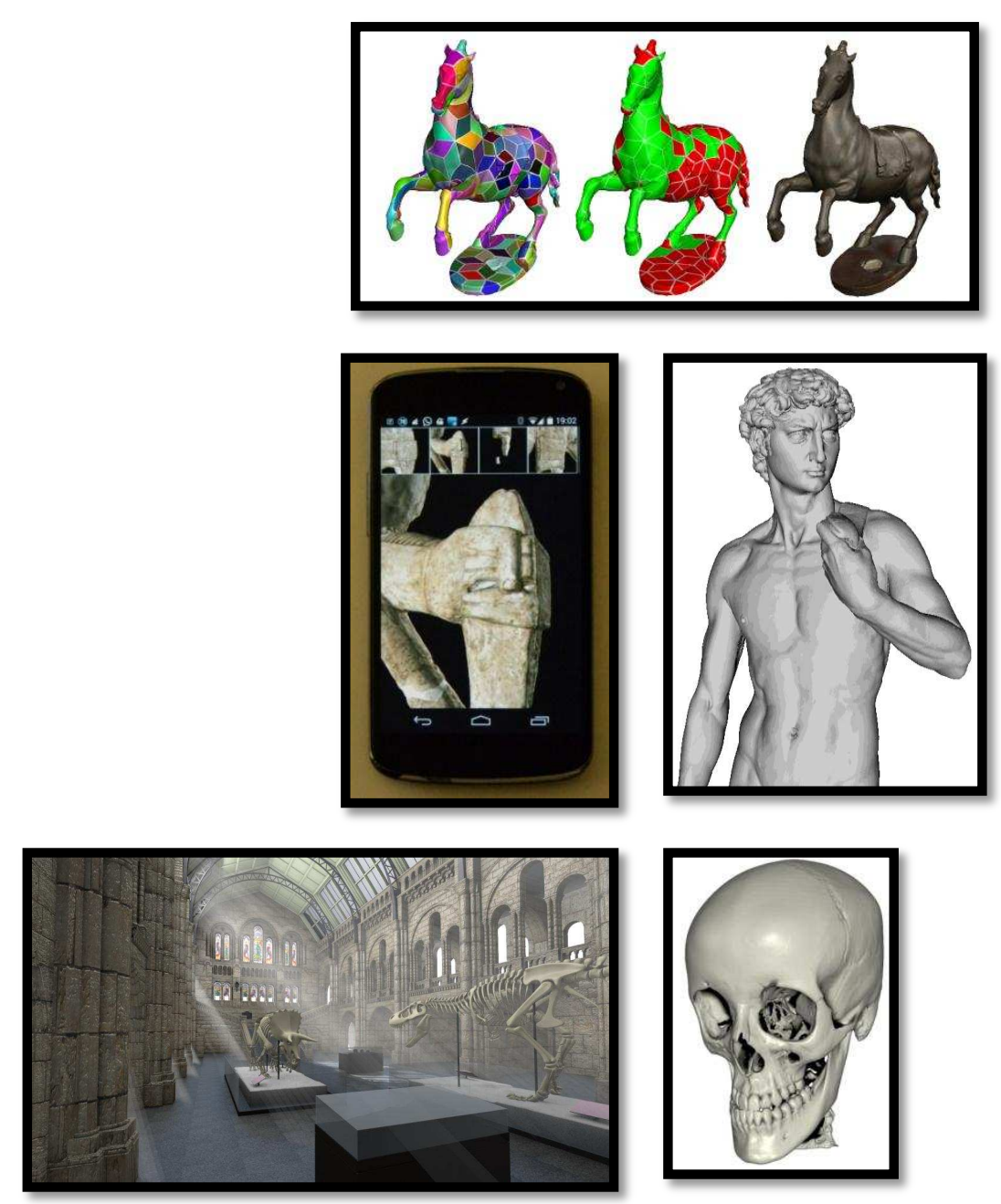


\section{Scalable Mobile Visualization. Outline}

Large meshes

High quality illumination: full precomputation

High quality illumination: smart computation

Volume data
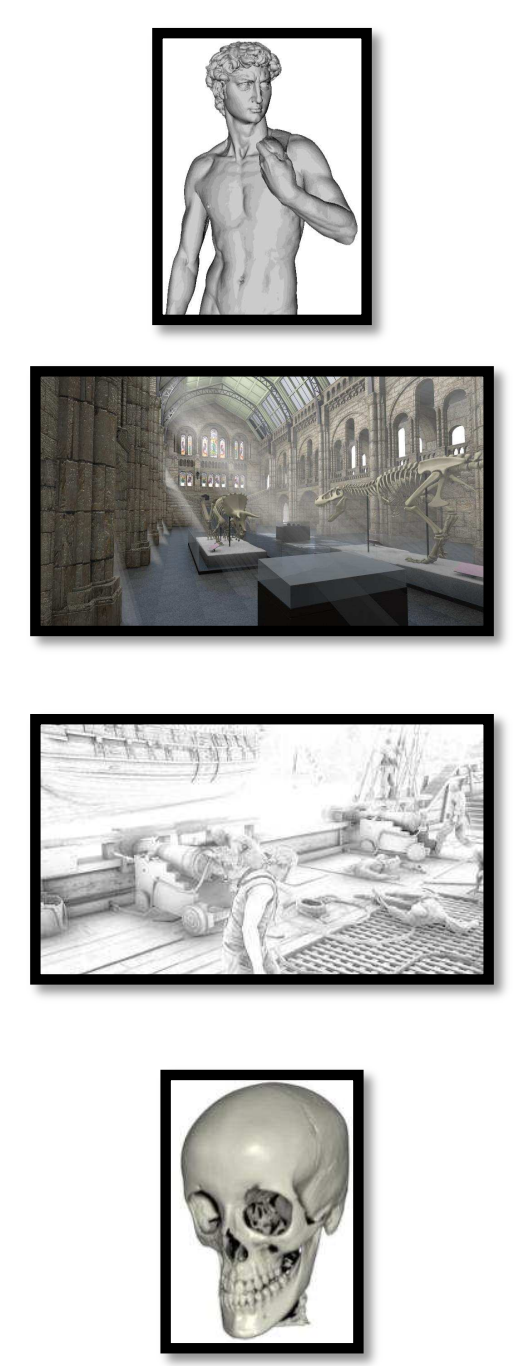


\section{Part 4.2}

\section{Scalable Mobile Visualization: Large Meshes}

Fabio Marton, CRS4 


\section{Scalable Mobile Visualization}

\section{Extremely}

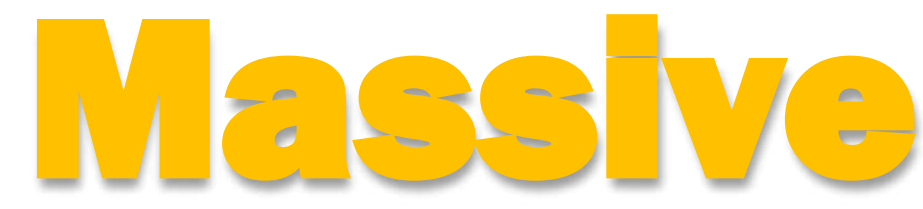

3D Models

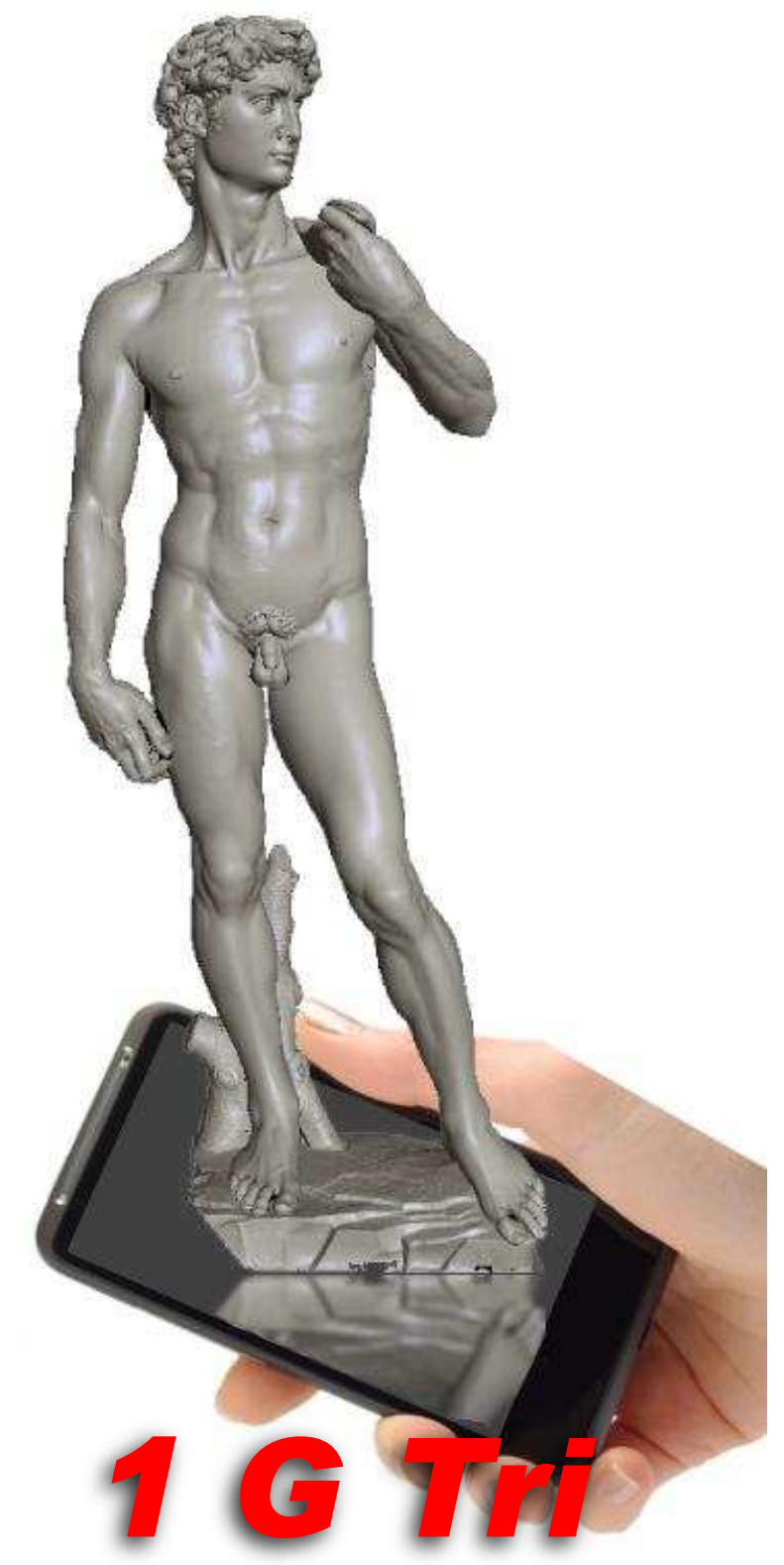




\section{Scalable Mobile Visualization}

Itty bitty living space! 


\section{A real-time data filtering problem!}

- Models of unbounded complexity on limited computers

- Need for output-sensitive techniques $(\mathrm{O}(\mathrm{N})$, not $\mathrm{O}(\mathrm{K}))$

- We assume less data on screen $(\mathrm{N})$ than in model $(\mathrm{K} \rightarrow \infty)$

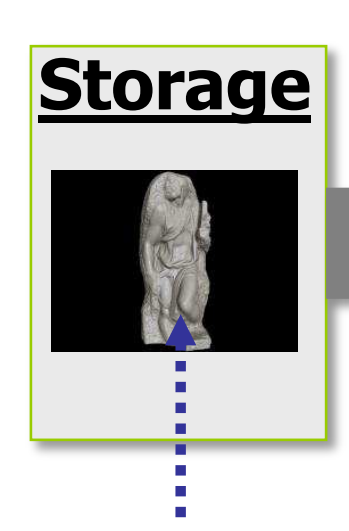

$\underline{\mathrm{O}(\mathrm{K}=\text { unbounded }) \text { bytes }}$

(triangles, points, ...)

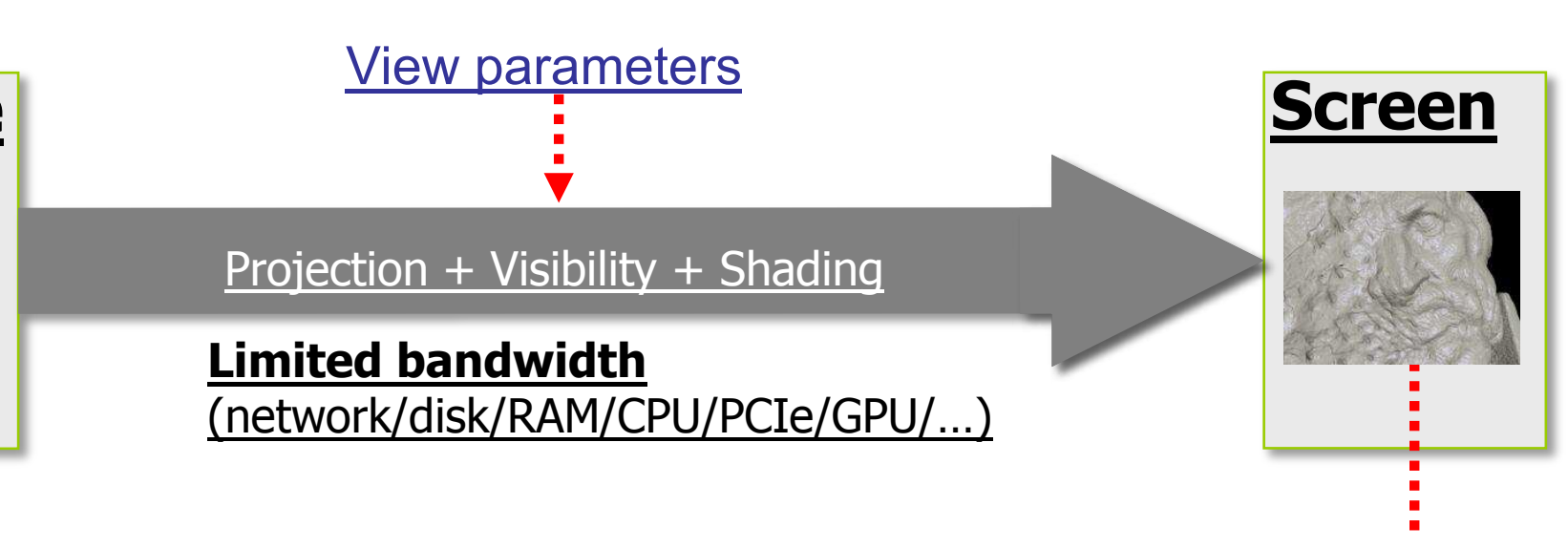

$10-100 \mathrm{~Hz}$

$\underline{\mathrm{O}(\mathrm{N}=1 \mathrm{M}-100 \mathrm{M}) \text { pixels }}$ 


\section{A real-time data filtering problem!}

- Models of unbounded complexity on limited computers

- Need for output-sensitive techniques $(\mathrm{O}(\mathrm{N})$, not $\mathrm{O}(\mathrm{K}))$

- We assume less data on screen $(\mathrm{N})$ than in model $(\mathrm{K} \rightarrow \infty)$

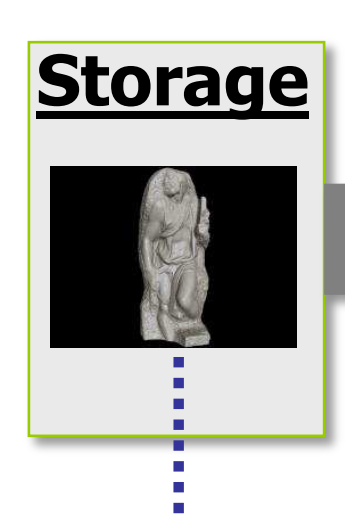

$\underline{\mathrm{O}(\mathrm{K}=\text { unbounded }) \text { bytes }}$

(triangles, points, ...)

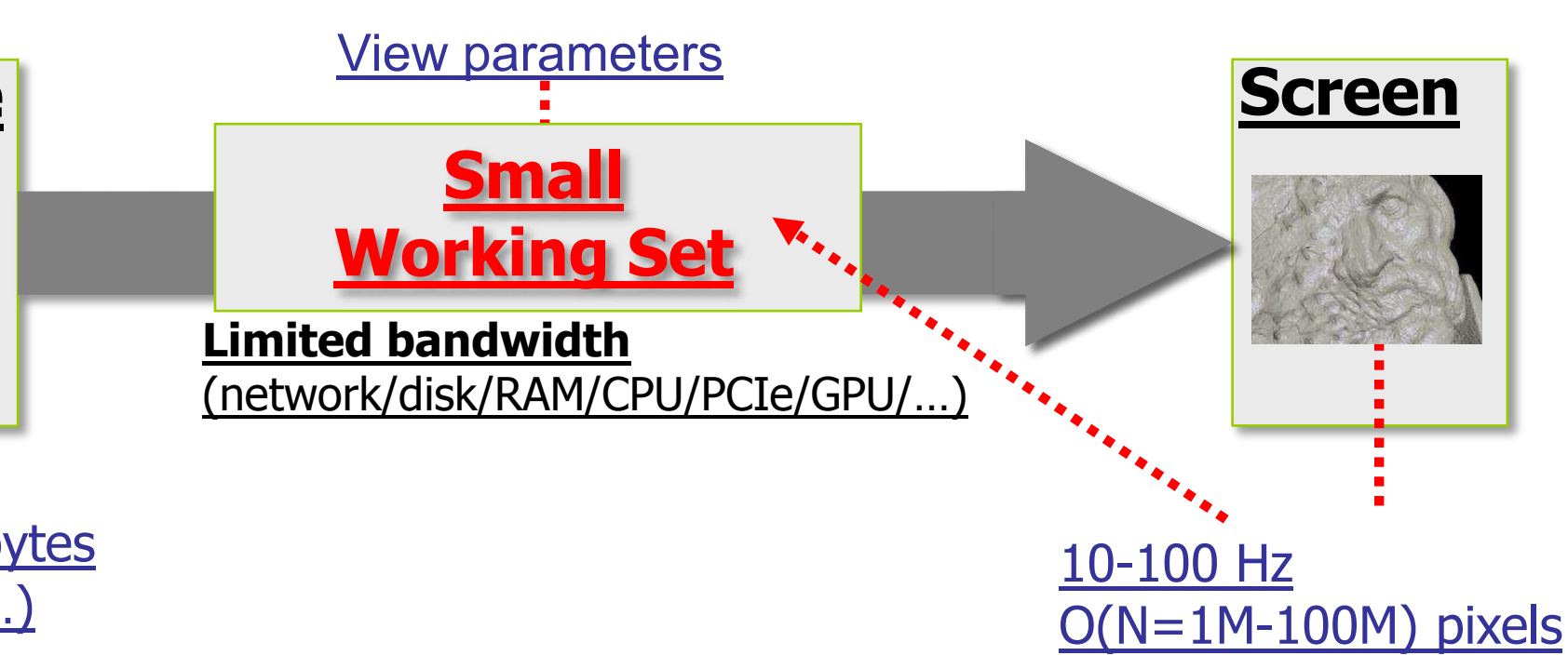




\section{Output-sensitive techniques}

- At preprocessing time: build MR structure

- Data prefiltering!

- Visibility + simplification

- Compression

- At run-time: selective view-dependent refinement from out-of-core data

- Must be output sensitive

- Access to prefiltered data under real-time constraints

- Visibility + LOD

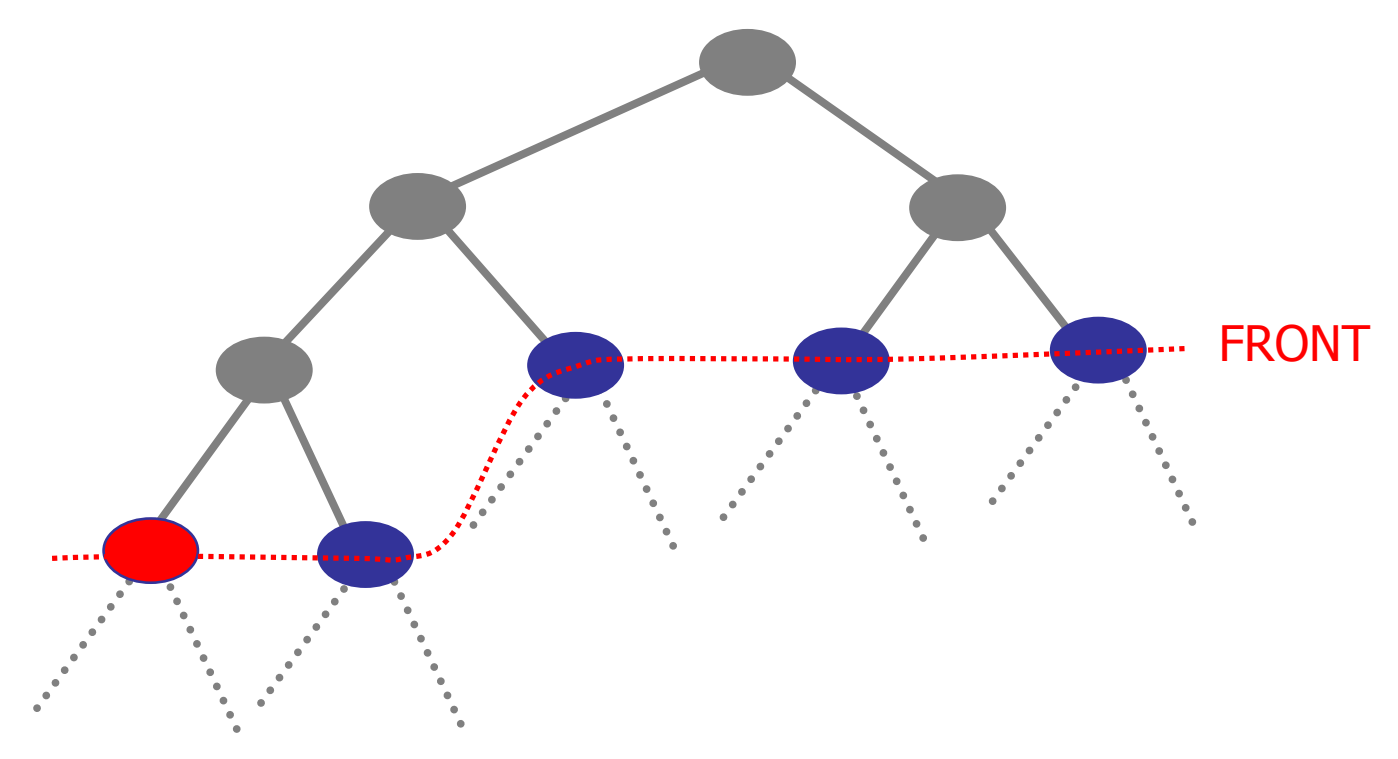




\section{Related work}

- Long history, starting with general solutions

- View dependent LOD and progressive streaming [Hoppe 1997]

- Compute view dependent triangulation each frame -> CPU bound

- Surface patches [CRS4+ISTI CNR, SIGGRAPH'04]

- Effective in terms of speed

- Require non-trivial data structures and techniques for decompression

- General solutions available for Desktop environments [Cignoni et al, 2005, Yoon et al. 2008]

- Mesh compression - MPEG-4 [Jovanova et al. 2008]

- Light 3D model rendering [MeshPad, PCL]

- Gigantic point clouds on mobile devices [Balsa et al. 2012]

- ... and much more 


\section{Our Contributions: chunked multiresolution structures}

\section{- Efficient view-dependent meshes}

- Approximate original surface

- Seamless

- Mix and match chunks

- Amortize CPU work!

\section{- Two approaches}

- Fixed coarse subdivision

- Adaptive QuadPatches

- Adaptive coarse subdivision

- Compact Adaptive TetraPuzzles
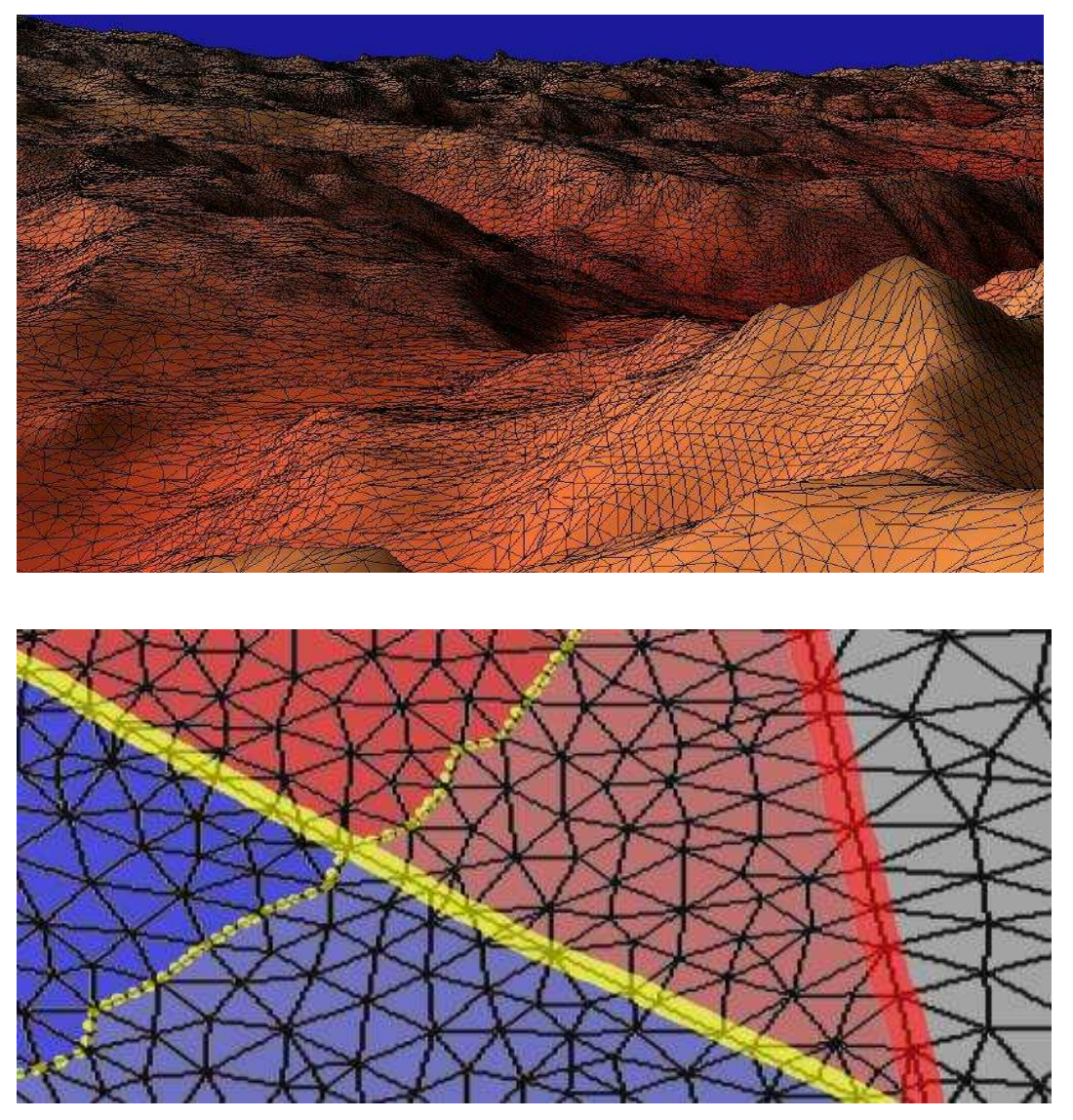


\section{Adaptive Quad Patches} Simplified Streaming and Rendering for Mobile \& Web

- Represent models as fixed number of multiresolution quad patches

- Image representation allows component reuse!

- Natural multiresolution model inside each patch

- Adaptive rendering handled totally within shaders!

- Works with topologically simple models

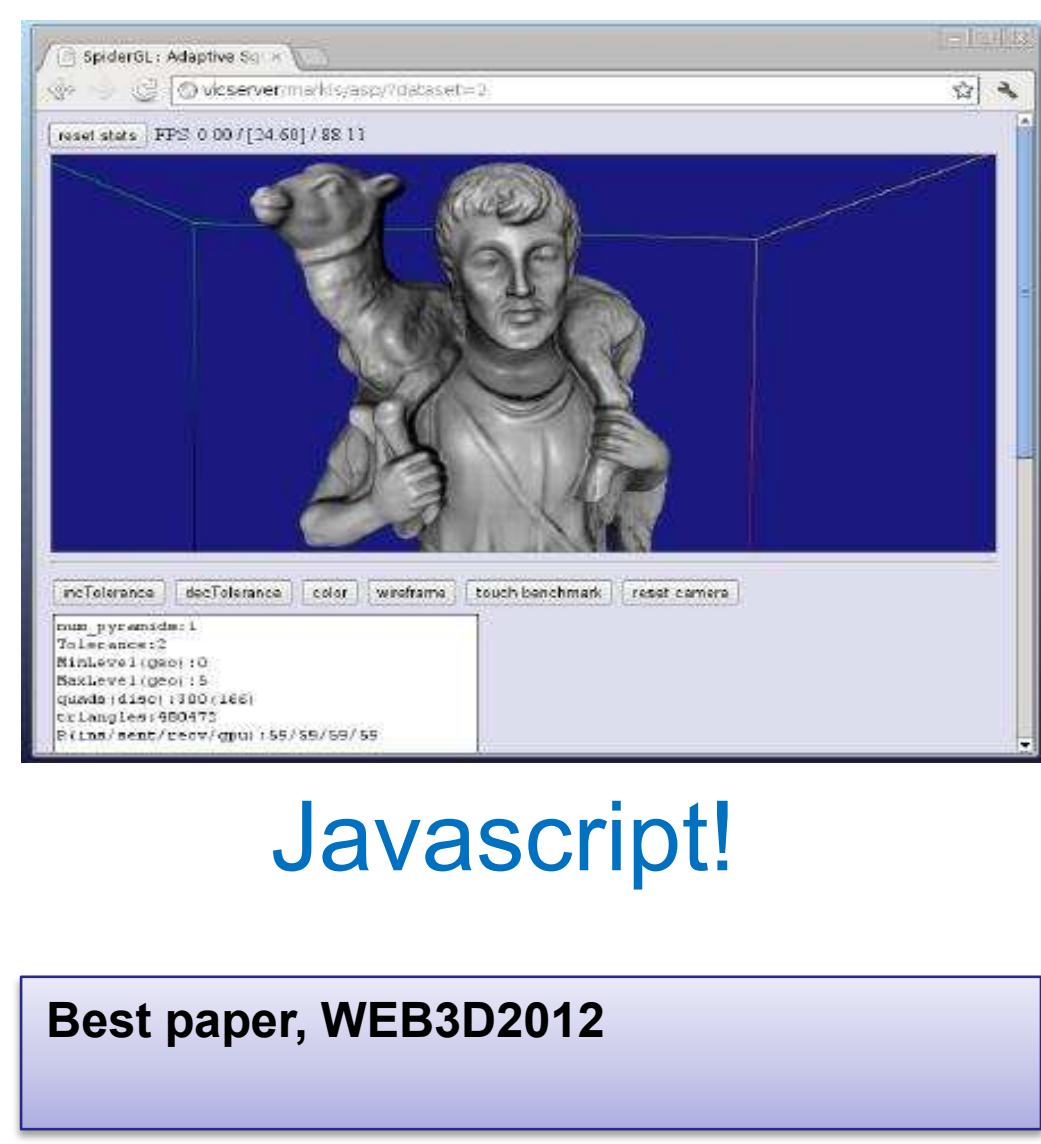




\section{Related work Adaptive Quad Patches}

- Geometry images [Gu et al. 2002]

- Exploit current GPU capabilities / optimized libraries for compression and streaming of images

- Quad remeshing

- Single-disk parametrization [Floater and Hormann 2005]

- Base mesh to parametrize the model [Petroni et al. 2010]

- Detail rendering

- GPU raycasting [Oliveira et al. 2000]

- Displacement mapping in GPU [Shiue et al. 2005] 


\section{AQP Approach}

- Models partitioned into fixed number of quad patches

- Geometry encoded as detail with respect to the 4 corners interpolation

- For each quad: 3 multiresolution pyramids

- Detail geometry

- Normals

- Colors

- Data encoded as images

- Exploit .png (lossless compression)

- Ensure connectivity

- Duplicated boundary information

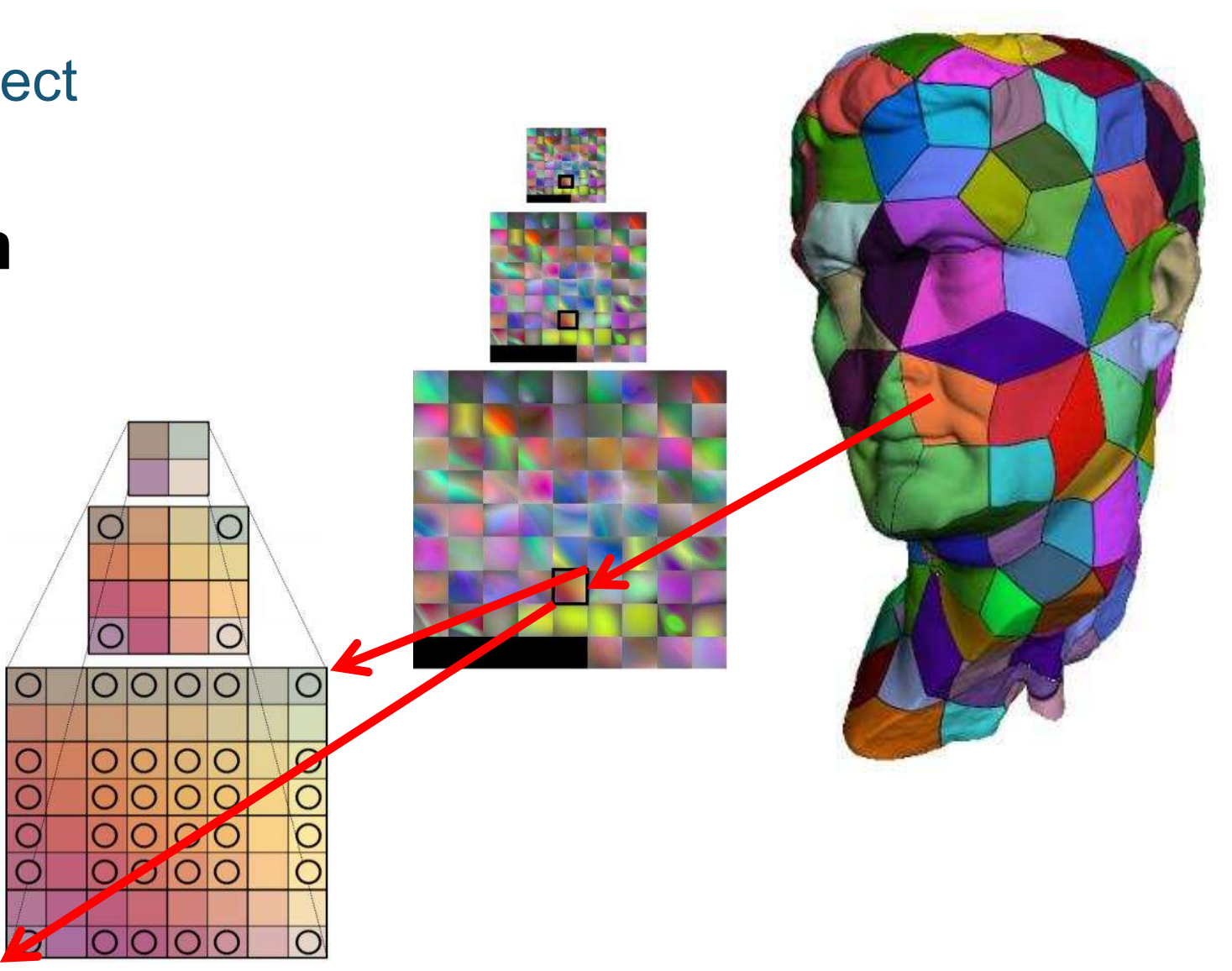




\section{Pre-processing (Reparameterization)}

- Generate clean manifold triangle mesh

-Poisson reconstruction [Kazhdan et al. 2006]

-Remove topological noise

- Discard connected components with too few triangles

- Parameterize the mesh on a quad-based domain

-Isometric triangle mesh parameterization

-Abstract domains [Pietroni et al. 2010]

-Remap into a collection of 2D square regions

- Resample each quad from original geometry

-Associates to each quad a regular grid of samples
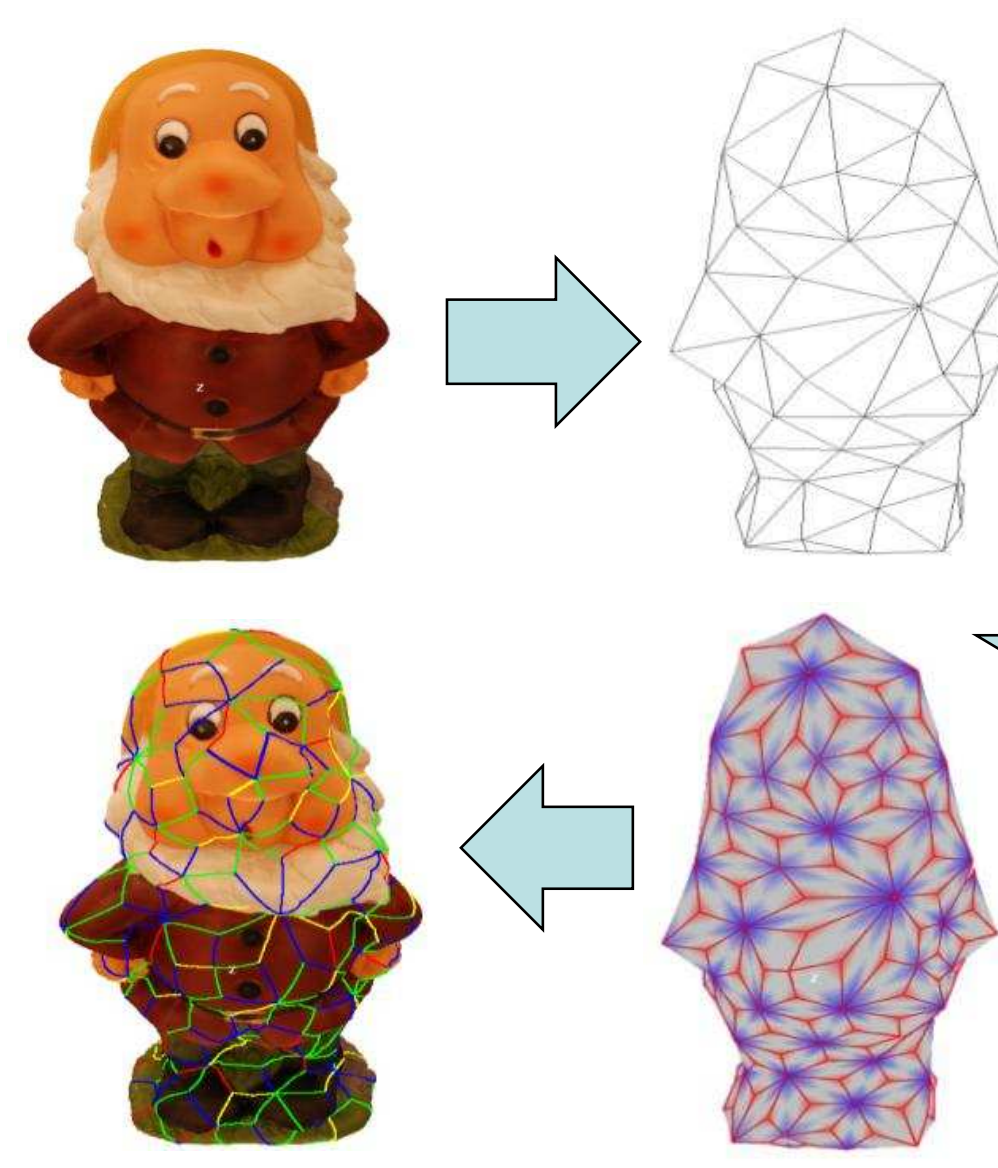

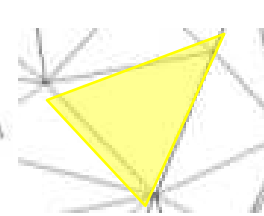

(position, color and normal) 


\section{Pre-processing (Multiresolution)}

- Collection of variable resolution quad patches

- Coarse representation of the original model

- Multiresolution pyramids

- Detail geometry

- Color

- Normals

- Shared border information

- Ensure connectivity

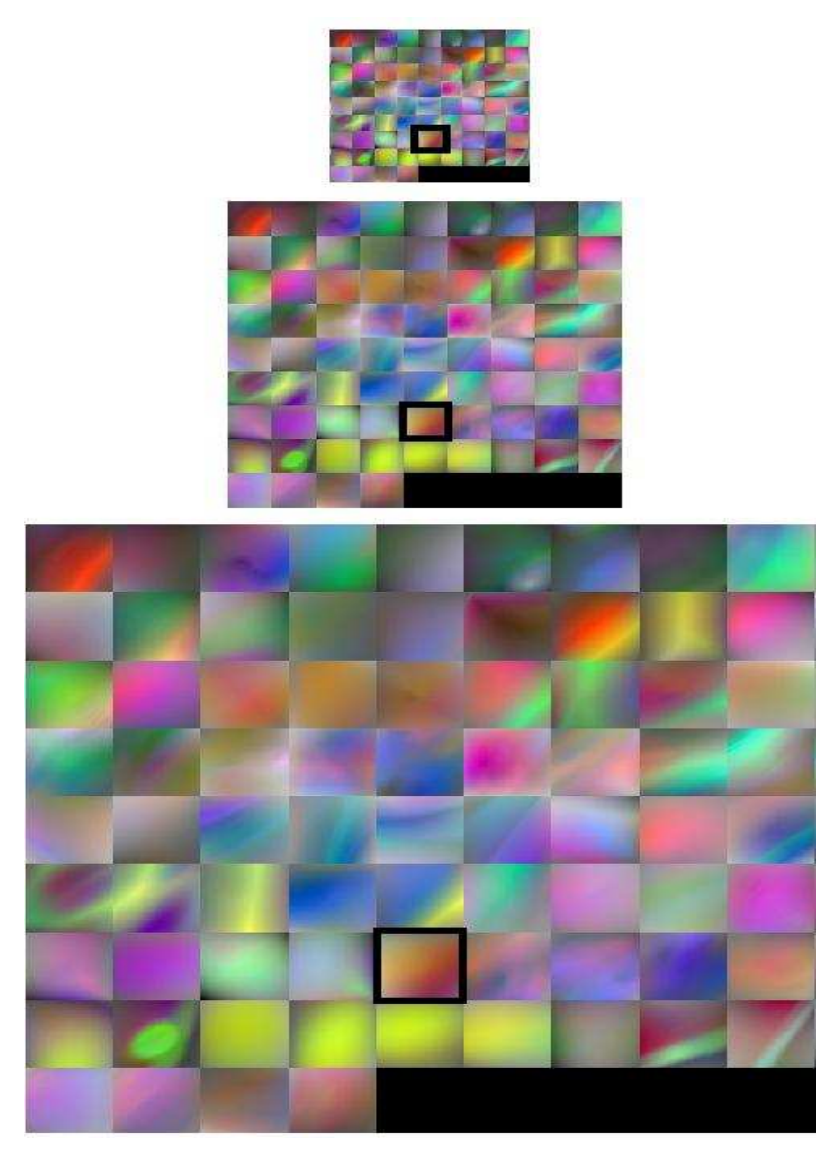




\section{Adaptive rendering}

\section{- CPU Lod Selection}

- Different quad LOD, but must agree on edges

- Quad LOD = max edge LOD (available)

- If LOD not available post asynchronous request, use finest

\section{- GPU drawing with Vertex Shader}

- Quad corners

- 1 VBO per resolution level reused $(u, v)$

- texture mipmaps of

- Displacements, Normals, Colors

- Texture with edge LODs (snap)

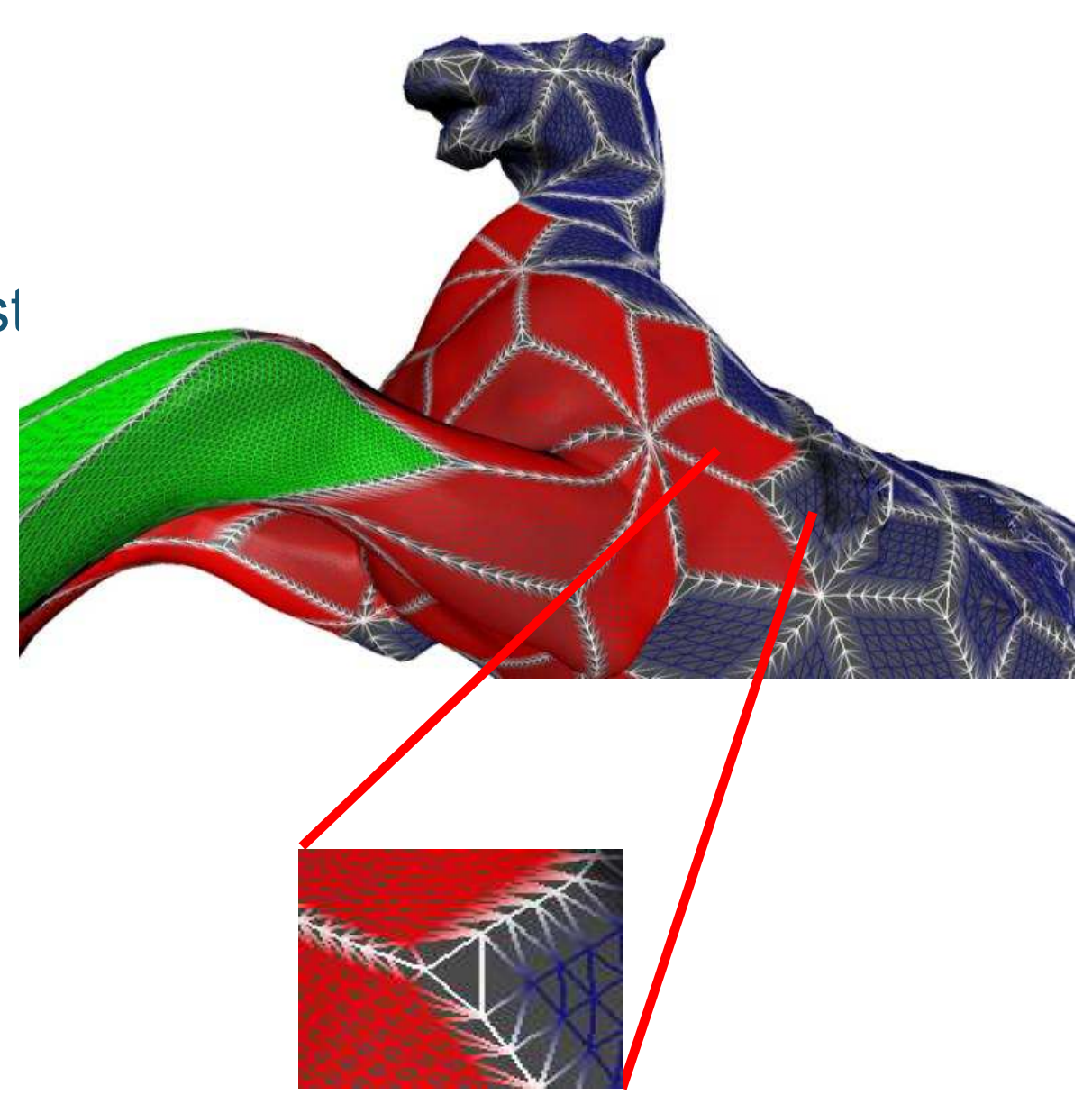


Rendering example

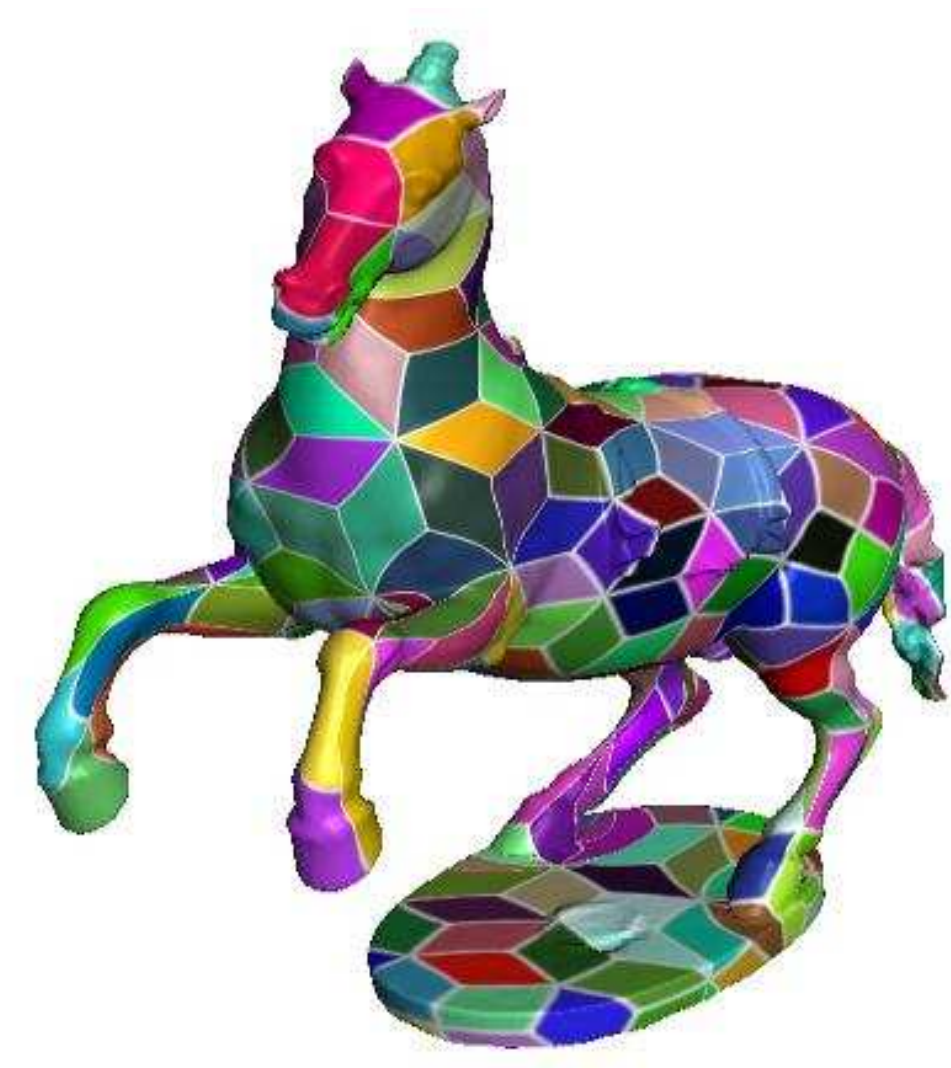

Patches

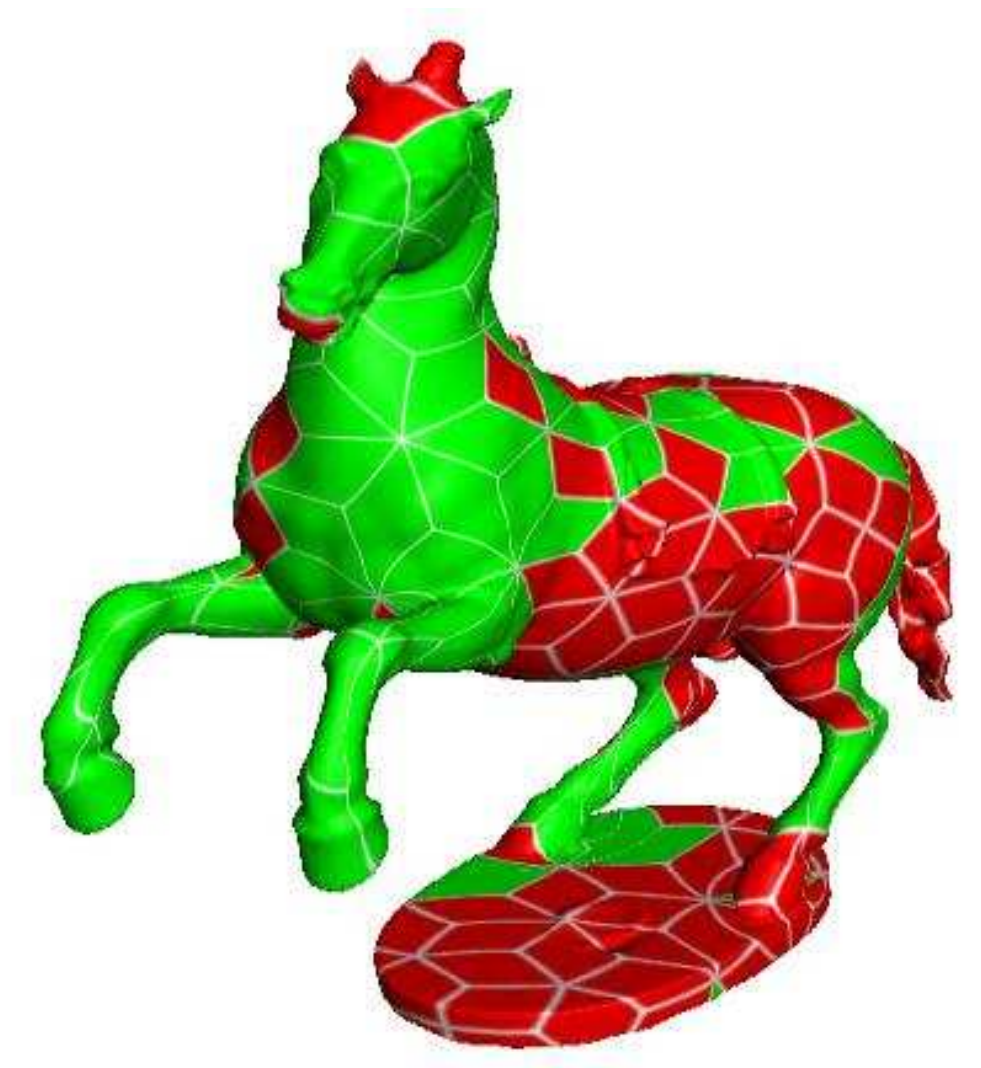

Shading

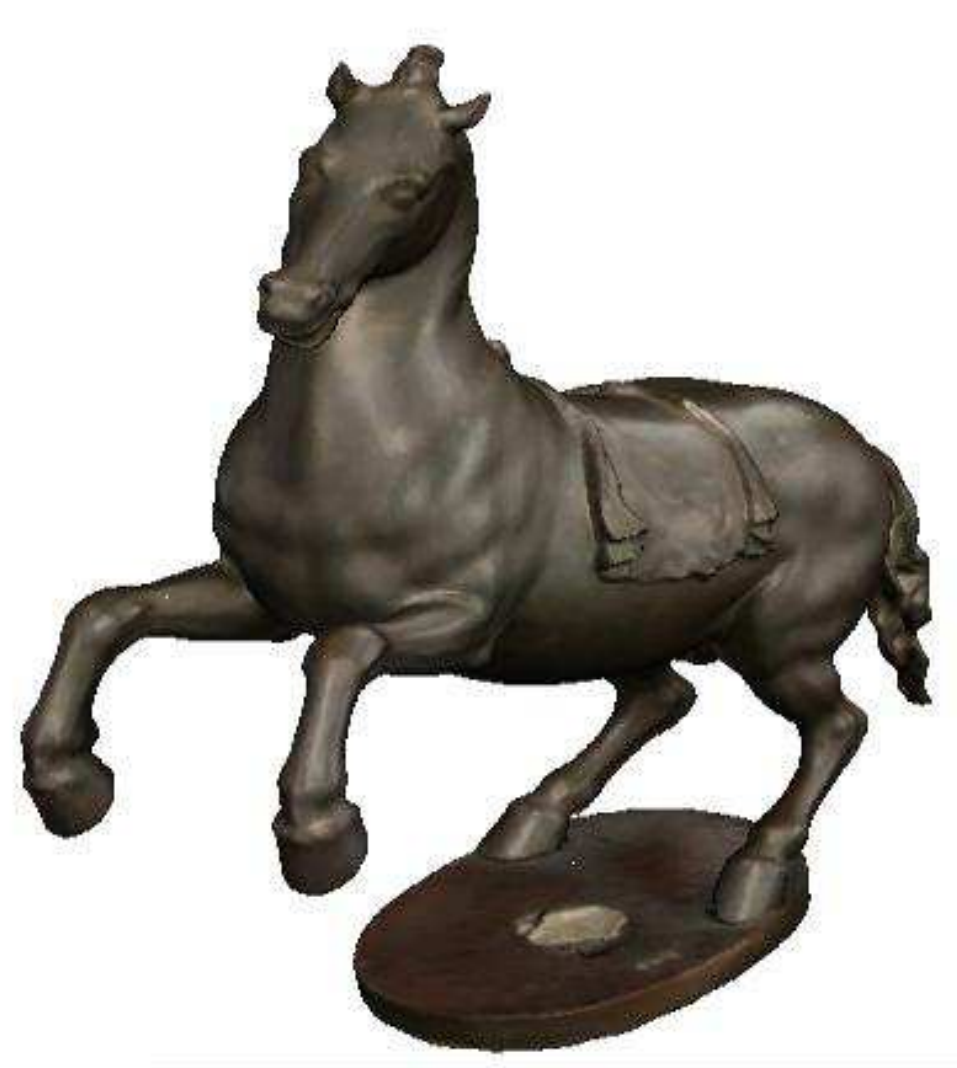

Levels

进 


\section{Adaptive rendering}

- 1. CPU LOD Selection

- Find edge LODs

- Quad LOD = max edge LODs

- If data available use it, otherwise

- Query data for next frames

- Use best available representation

- Send VBO with regular grid (1 for each LOD)

- 2. GPU: Vertex Shader

- Snap vertices on edges (match neighbors)

- Base position $=$ corner interpolation $(u, v)$

- Displace VBO vertices

- normal + displacement (dequantized)

\section{- 3. GPU: Fragment Shader}

- Texturing \& Shading

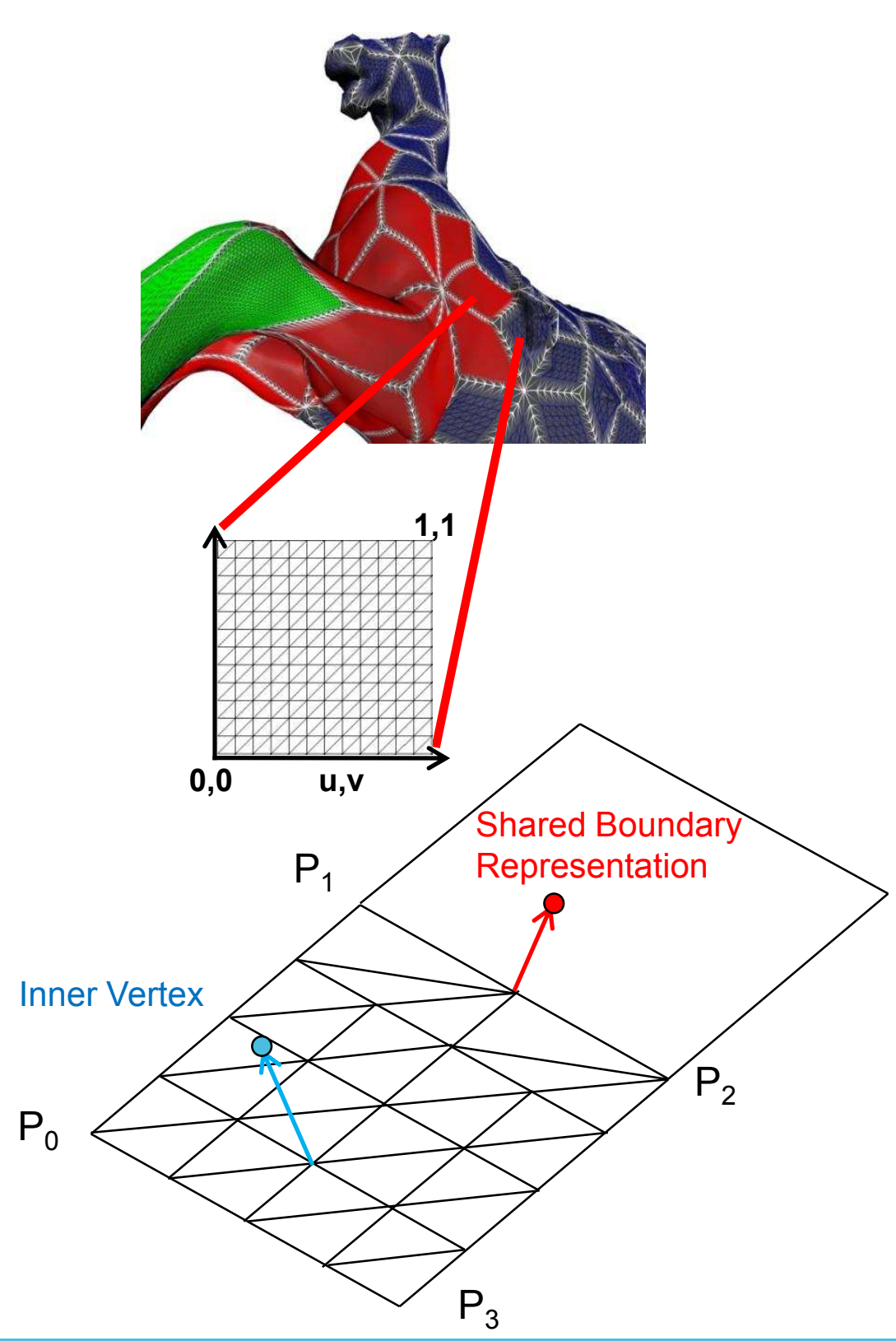

24 


\section{Results}
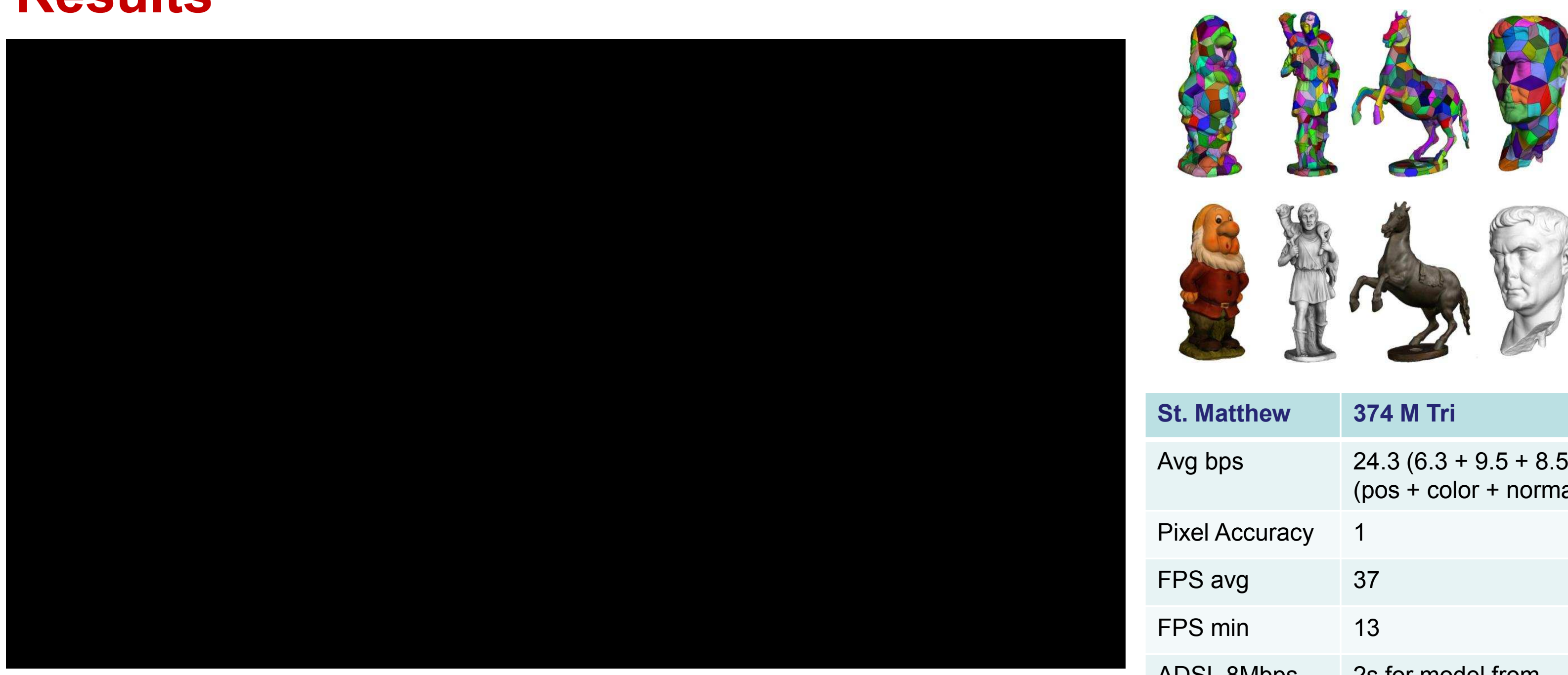

St. Matthew

Avg bps

Pixel Accuracy

FPS avg

FPS min

ADSL 8Mbps refine time
374 M Tri

$24.3(6.3+9.5+8.5)$ (pos + color + normal $)$ 1 37 13

$2 s$ for model from scratch 


\section{Adaptive Quad Patches Conclusions}

- Effective creation and distribution system

-Fully automatic

-Compact, streamable and renderable 3D model representations

-Low CPU overhead

- WebGL

- Desktop

- Mobile

\section{- Limitations}

-Closed objects with large components

-Visual approximation (lossy)

-Explore more aggressive compression techniques

-Occlusion culling

-More sophisticated shading/shadowing techniques

- Next: More general solution based on full multiresolution structure 


\section{Compact Adaptive TetraPuzzles}

Adaptive multiresolution solution with compression-domain rendering

- Represent models as variable number of multiresolution surface patches embedded in a hierarchy of tetrahedra

- Regular conformal hierarchy of tetrahedra spatially partitions input mesh

- Mesh fragments at different resolutions associated to implicit diamonds

- Fully adaptive and seamless 3D mesh structure with local quantization

- Geometry clipped against containing tetrahedra

- Barycentric coordinates used for local tetrahedra geometry reparameterization

- GPU friendly compact data representation

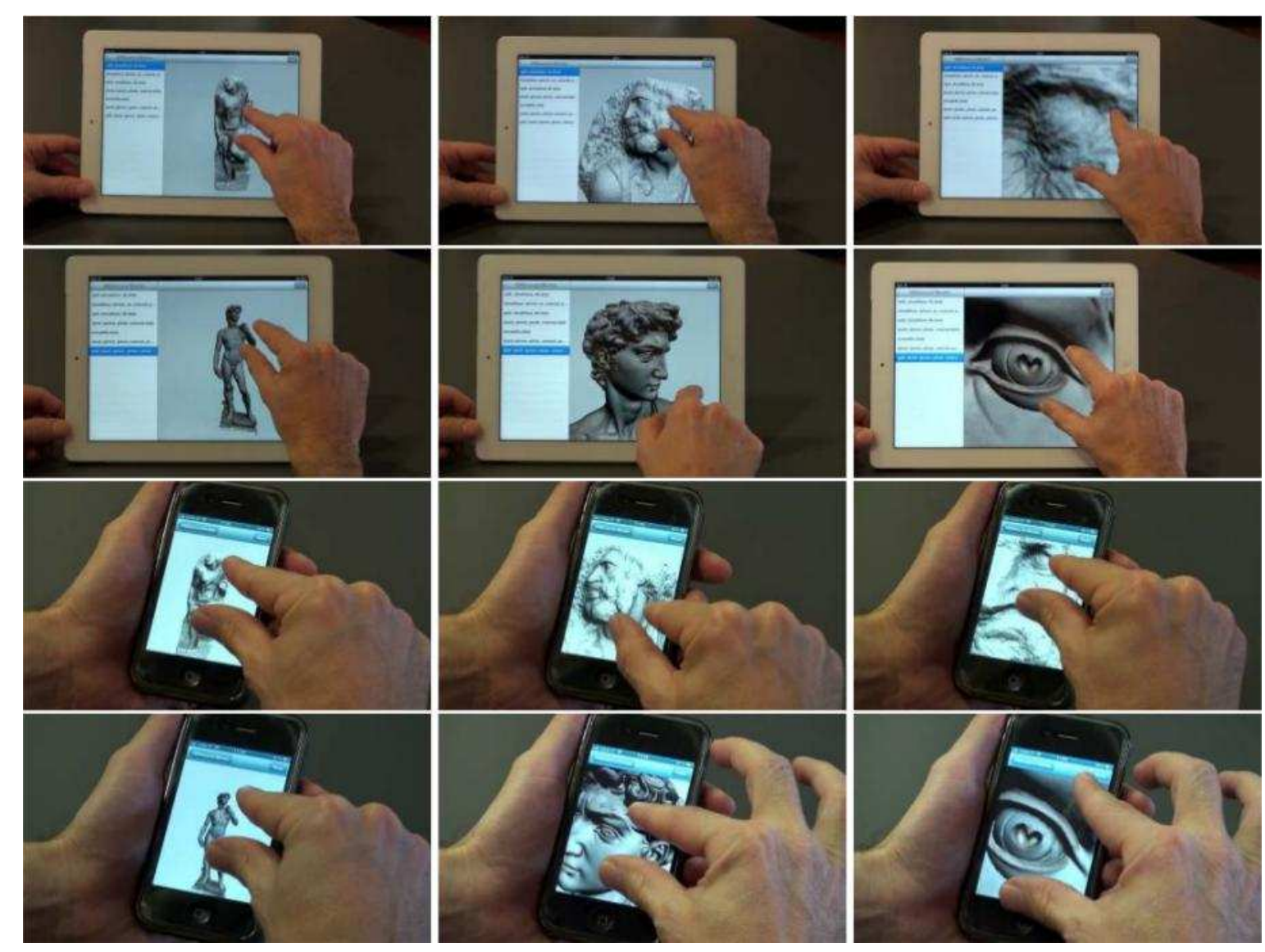

- Works with general surface models 


\section{Compact Adaptive Tetra Puzzles}

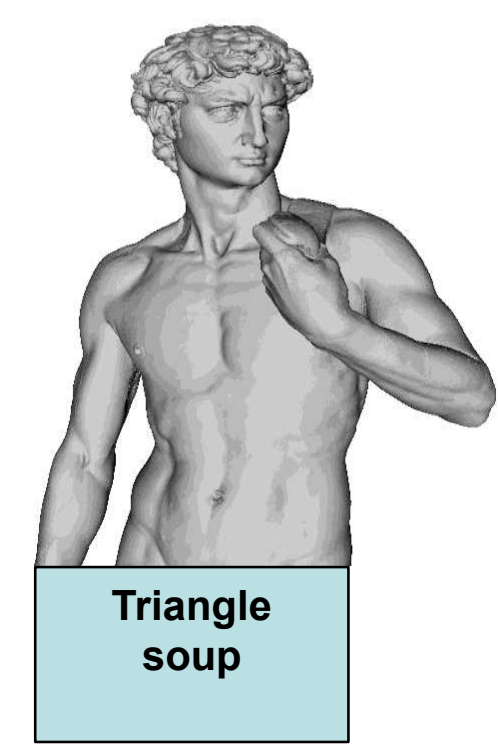

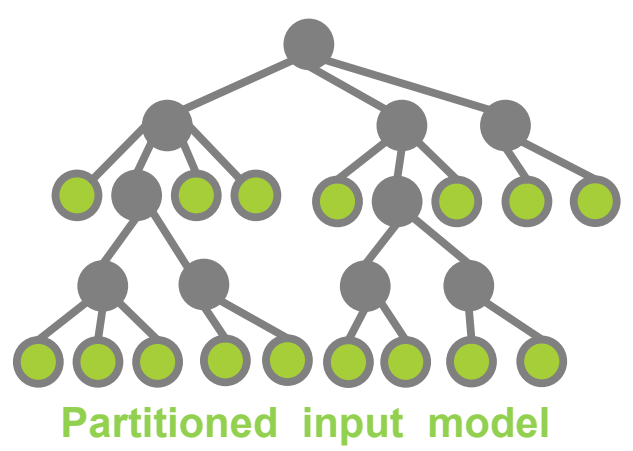

Tetrahedra hierarchy

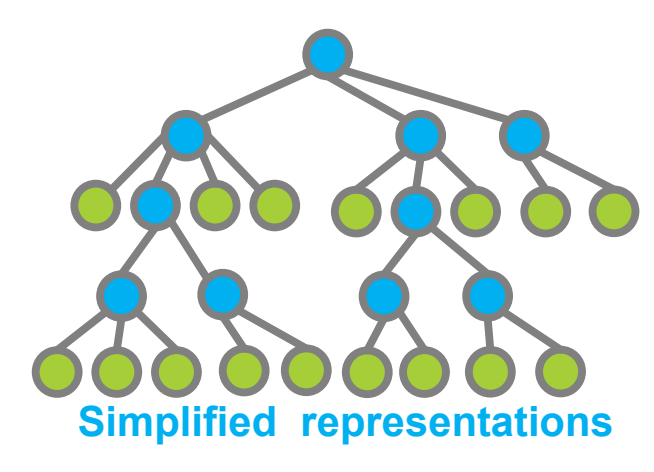

Tetrahedra hierarchy
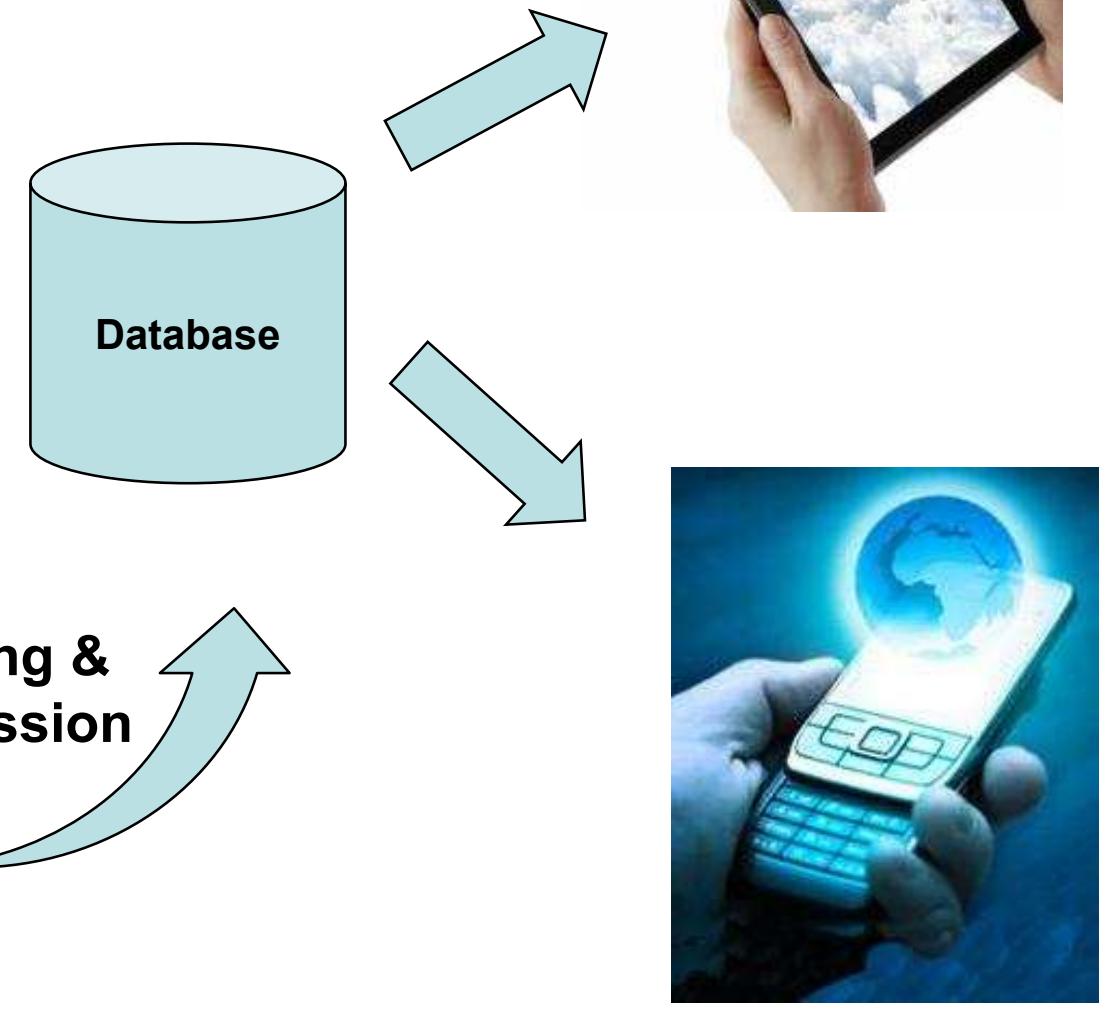
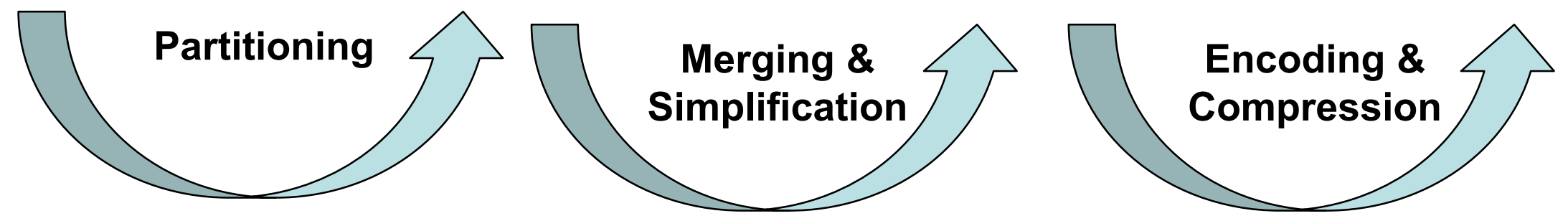


\section{Related work (Compression)}

- Topology coding

- Theoretical minimum [Rossignac 2001]

- $1.62 \mathrm{bits} /$ triangle, $3.24 \mathrm{bits} / \mathrm{vertex}$

- 8 bpt/16 bpv [Chhugani et al. 2007]

- HW-implementation

- 5 bpt/10 bpv [Meyer et al. 2012]

- CUDA implementation

- Attribute quantization

- Global position quantization [Lee et al. 2009]

- Local quantization techniques [Lee et al. 2010]

- Normal compression using octahedral parametrization [Meyer et al. 2010]

- Our goal is to balance compression rate and decoding+rendering performance by using a GPU-friendly compact representation 


\section{Overview}

- Construction

- Start with hires triangle soup

- Partition model

- Construct non-leaf cells by bottom-up recombination and simplification of lower level cells

- Assign model space errors to cells

- Rendering

- Refine graph

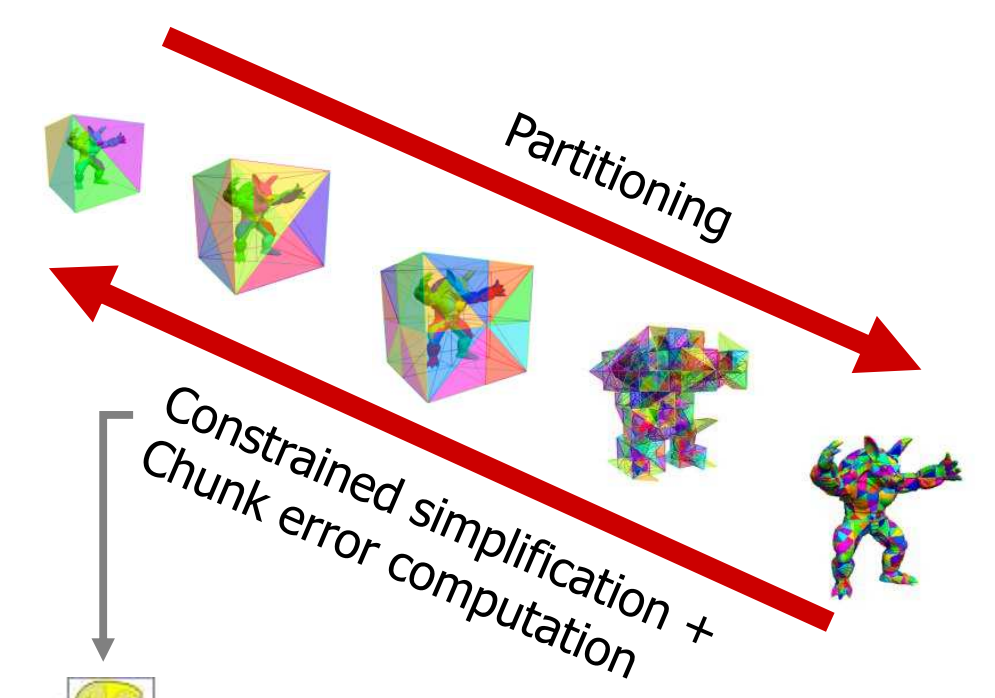

- Render selected precomputed cells

\section{Ensure continuity $\rightarrow$ Shared information on borders}




\section{Preprocessing}

- Geometry clipped against containing tetrahedra

- Vertices: tetrahedra barycentric coordinates

- Pbarycentric $=\lambda 1^{*} \mathrm{P} 1+\lambda 2^{*} \mathrm{P} 2+\lambda 3^{*} \mathrm{P} 3+\lambda 4^{*} \mathrm{P} 4$

- Seamless local quantization

- Inner vertices (I): 4 corners

- Face vertices $(F): 3$ corners

- Edge vertices (E): 2 corners

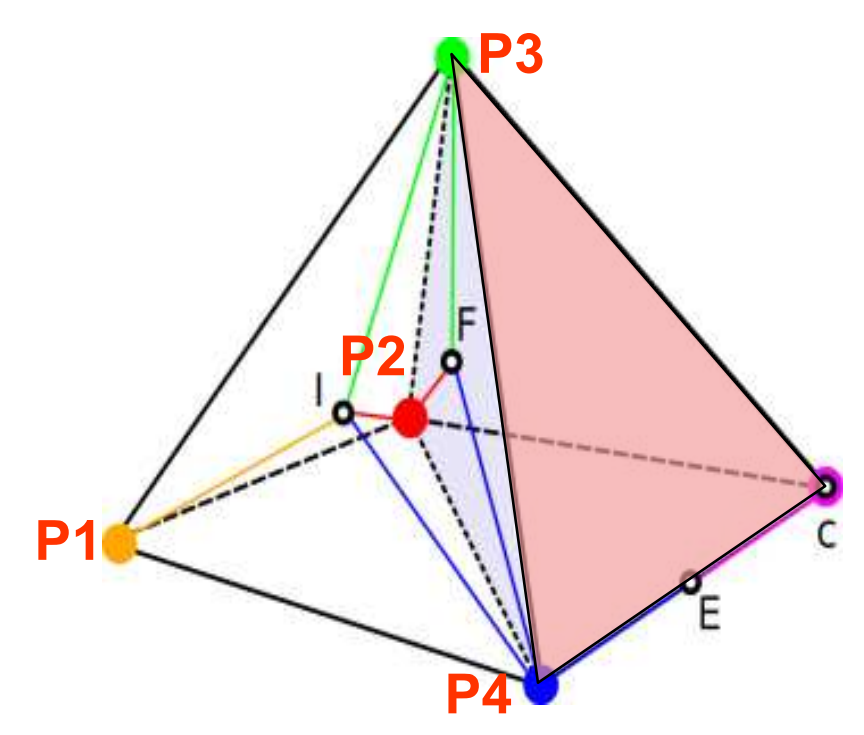

- GPU friendly compact data representation

-8 bytes $=$ position $(3$ bytes $)+\operatorname{color}(3$ bytes $)+$ normal $(2$ bytes $)$

- Normals encoded with the octahedron approach [Meyer et al. 2012]

- Further compression with entropy coding

- exploiting local data coherence

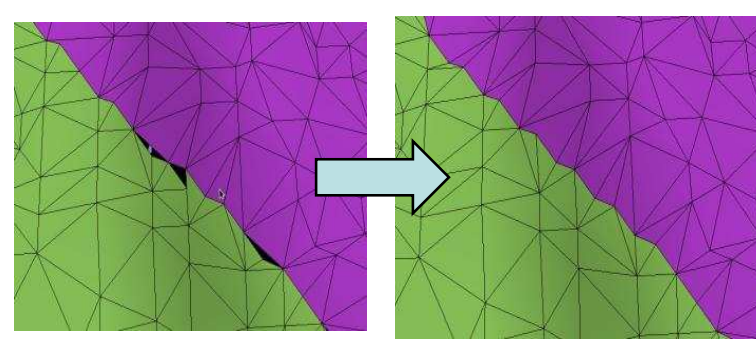




\section{Rendering process}

- Extract view dependent diamond cut (CPU)

- Request required patches to server

- Asynchronous multithread client

- Apache 2 based server (data repository, no processing)

- CPU entropy decoding of each patch - For each node (GPU Vertex Shader):
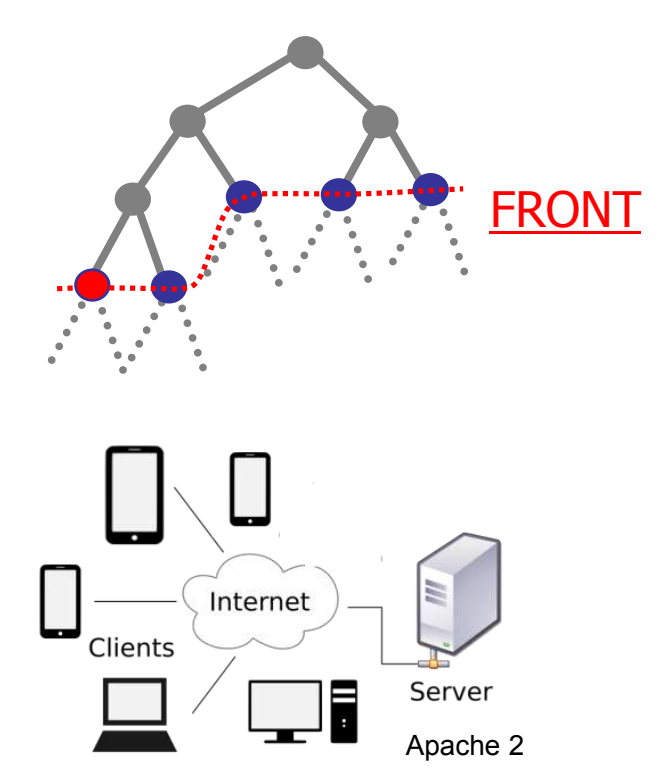

- VBO with barycentric coordinates, normals and colors (64 bpv)

- Decode position : $\mathrm{P}=\mathrm{MV} *{ }^{*}[\mathrm{C} 0 \mathrm{C} 1 \mathrm{C} 2 \mathrm{C} 3]^{*}[\mathrm{Vb}]$

- $\mathrm{Vb}$ is the vector with the 4 barycentric coords

- C0..C3 are tetrahedra corners

- Decode normal from 2 bytes encoding [Meyers et al. 2012]

- Use color coded in RGB24

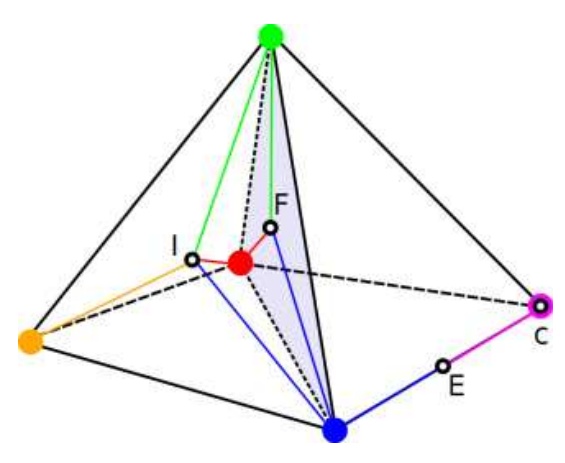




\section{Results}

- Input Models

- St. Matthew 374 MTri

- David 1GTri

- Compression:

- 40 to 50 bits/vertex

- Streaming full screen view

- 30 s on wireless,

- $45 \mathrm{~s}$ on $3 \mathrm{G}$

- David 14.5MB (1.1 Mtri)

- St. Matthew 19.9MB (1.8 Mtri)

Rendering

Pixel tolerance

Triangle throughput

FPS avg

FPS refined views

Triangle Budget

iPad gen3

3

$30 \mathrm{Mtri} / \mathrm{s}$

35

15

$2 \mathrm{M}$

\section{iPhone 4} 3

2.8 Mtri/s 10

2.8

$1 \mathrm{M}$ 


\section{Conclusions: Compact ATP}

- Generic gigantic 3D triangle meshes on common handheld devices

- Compact, GPU friendly, adaptive data structure

- Exploiting the properties of conformal hierarchies of tetrahedra

- Seamless local quantization using barycentric coordinates

- Two-stage CPU and GPU compression

- Integrated into a multiresolution data representation

- Limitations

- Requires coding non-trivial data structures

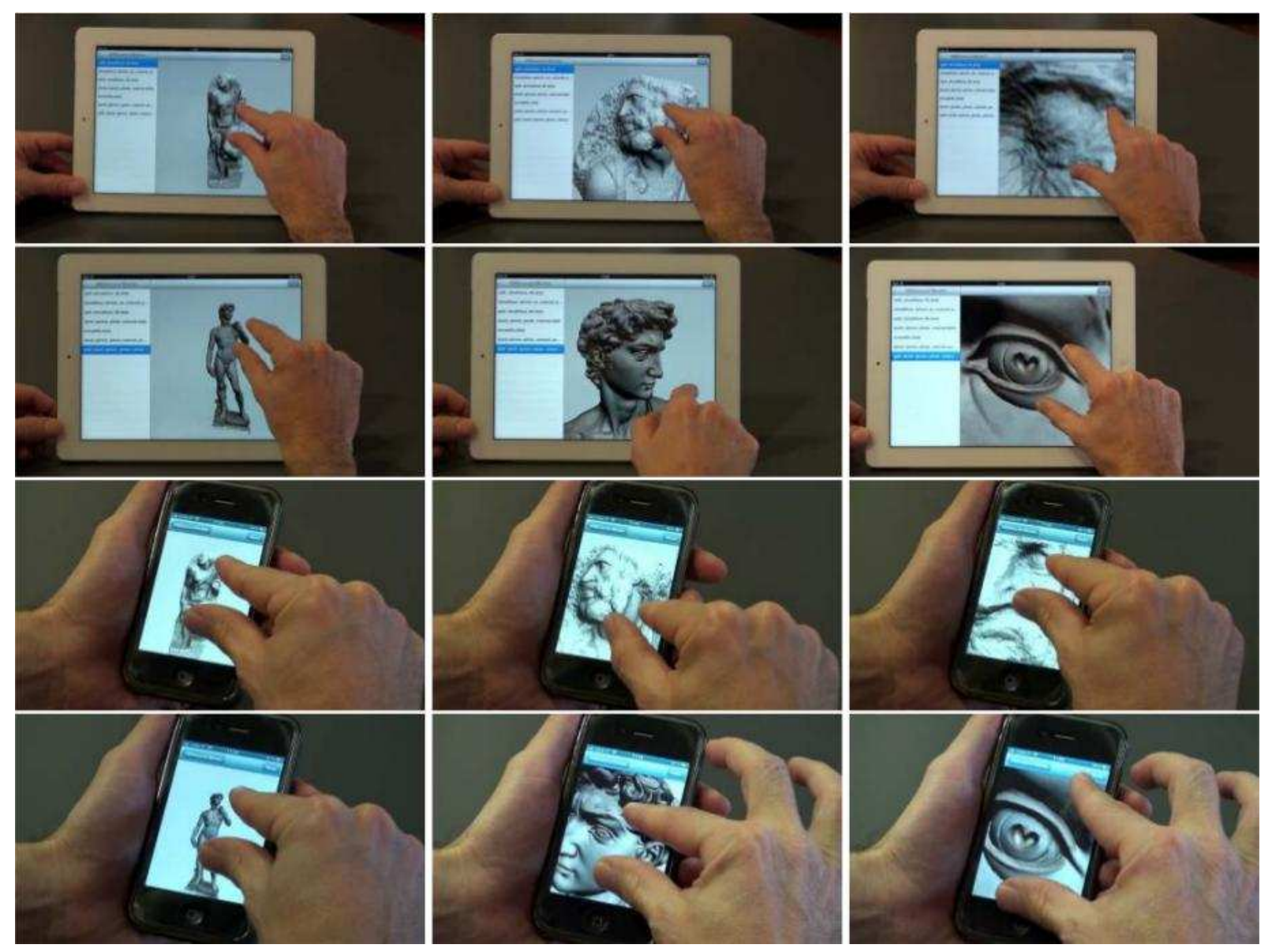

- Hard to implement on scripting environments 


\section{Conclusions: large meshes}

- Various solutions for large meshes

- Constrained solution: Adaptive Quad Patches

- Simple and fast

- Good compression

- Works on topologically simple models

- General solution: Compact Adaptive Tetra Puzzles

- Compact data representation

- More complex code 


\section{Part 4.3}

\section{Scalable Mobile Visualization: \\ Introduction to complex lighting}

\section{Enrico Gobbetti, CRS4}




\section{Complex scenes}

- We have seen how to deal with complex meshes O(Gtri)

- Similar solutions for point clouds...

- Problem tackled was size

- Solution proposed: adaptive multiresolution chunk-based approaches

- Various optimized solutions to select chunks, compose them, ...

- Rendering was simple, though

- One pass streaming, direct illumination

- How to deal with more complex illumination and shading? 


\section{Complex scenes}

- Complex illumination/shading introduce data and computation problems

- Non-local effects (global illumination, shadows, ...) require scattered information

- Illumination/shading is costly (CPU/GPU time) and requires data-intensive algorithms

- Proposed solutions in the mobile world

- Full precomputation

- Images computed off-line

- Removes real-time timing constraints, but introduces other problems (which images to compute? How to navigate in an image-based scene?)

- Smart computation

- Partial precomputation of some intermediate results, approximation tricks

- Not general solution but improves quality!

- Next session illustrates examples of full/smart computation in mobile graphics 


\section{Part 4.4}

\section{Scalable Mobile Visualization:}

Full precomputation of complex lighting

\section{Fabio Marton, CRS4}




\section{Ubiquitous exploration of scenes with complex illumination}

- Real-time requirement: $\sim 30 \mathrm{~Hz}$

- Difficulties handling complex illumination on mobile/web platforms with current methods

- Image-based techniques

- Constraining camera movement to a set of fixed camera positions

- Enable pre-computed photorealistic visualization

- Explore-Maps: technique for

- Scene representation as set of probes and arcs

- Precomputed rendering for probes and transitions
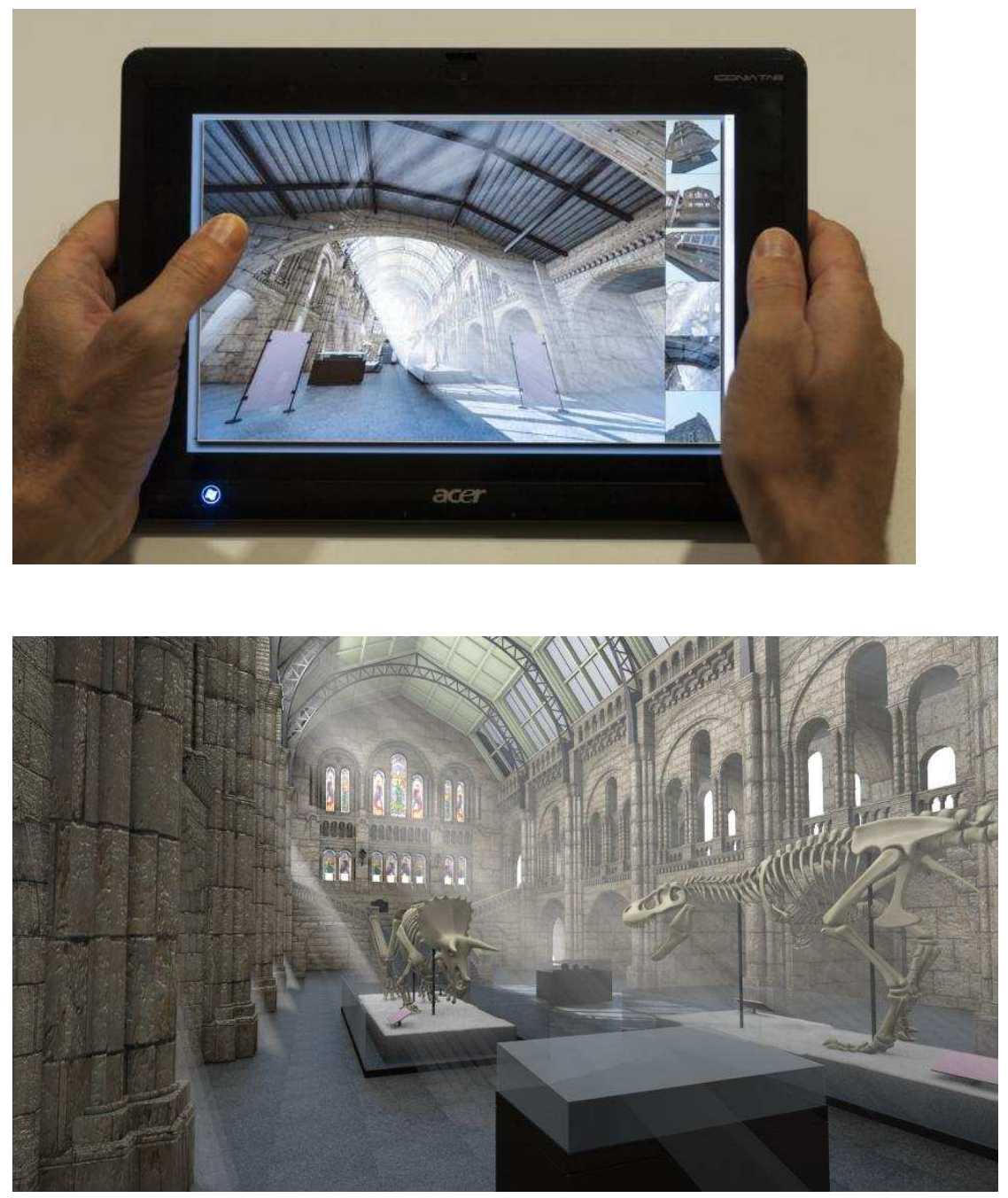


\section{Scene Discovery}

- ExploreMaps: Automatic best view/best path methods for generating

- Set of probes providing full model coverage

- Probe $=360^{\circ}$ panoramic point of view

Explore Map

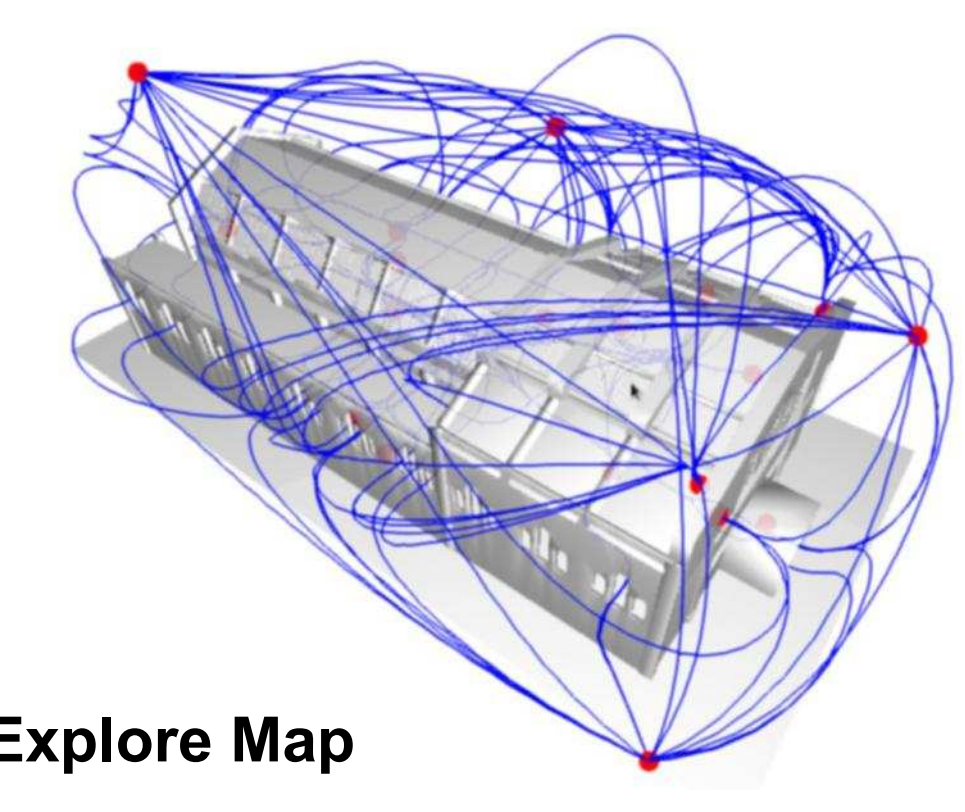

- Set of arcs connecting probes

- Enable full scene navigation

Di Bendeetto et al. Eurographics 2014

ExploreMaps: Efficient Construction and Ubiquitous Exploration of Panoramic View

Graphs of Complex 3D Environments.

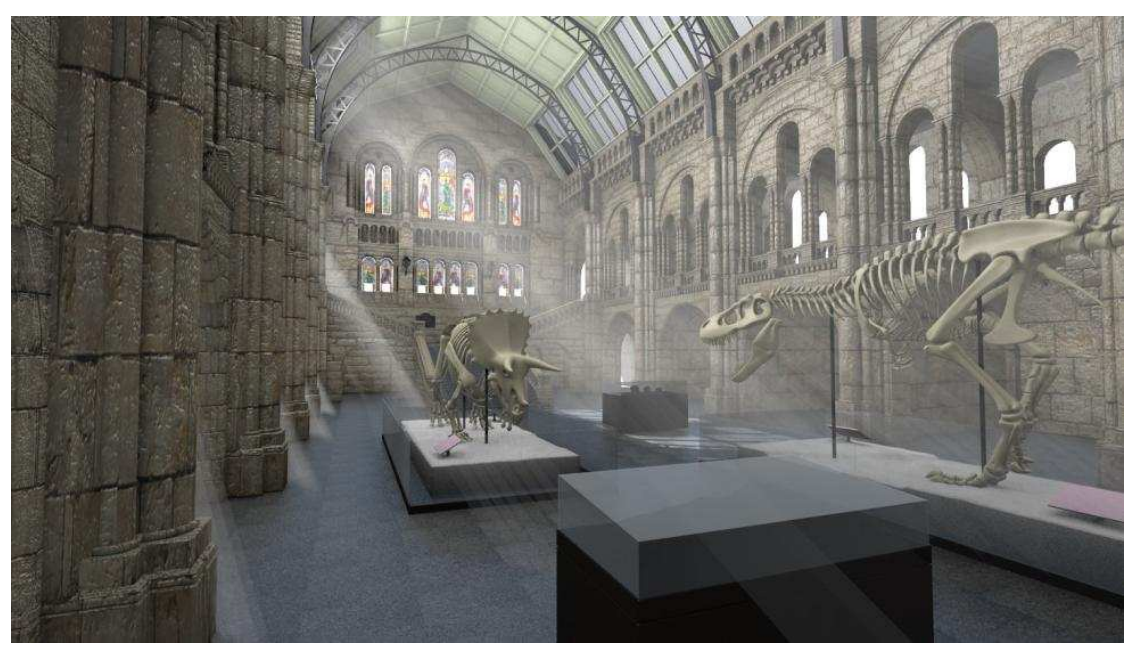




\section{Best viewpoints computation}

- Position set of probes inside the scene

- Probes provide a 360 degree view

- Greedy algorithm that places probes at the barycenter of newly seen geometry until all the scene is visible

- Final clustering pass reduces number of probes
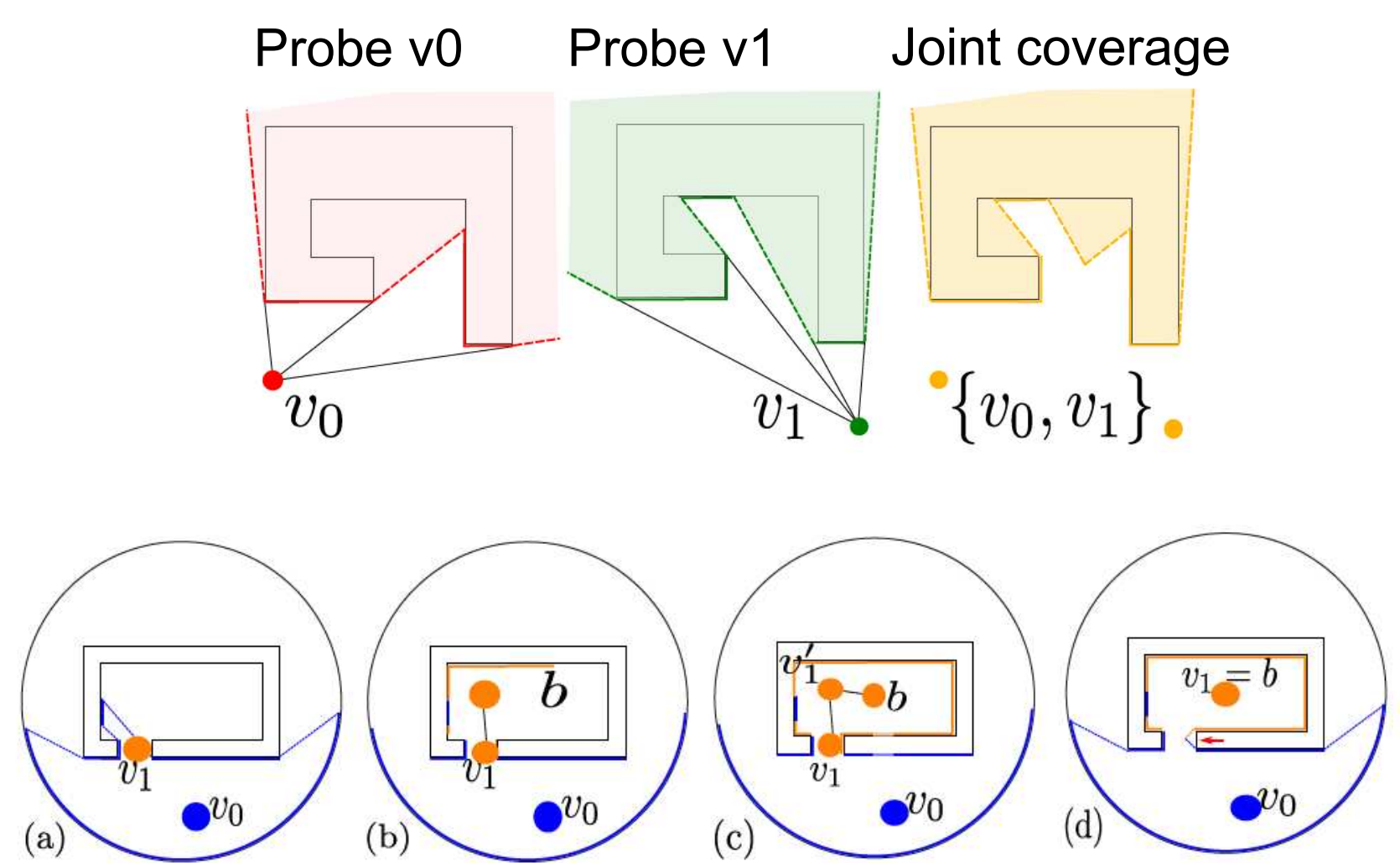

Coverage optimization, by moving to the barycenter of seen geometry 


\section{Best path computation}

- Connect probes which have a common visible region

- Creates a graph of probes

- For each pair of mutually visible probe

- Create first path going through the closest point in the mutually visible region

- Optimize and smooth the path using a mass-spring system
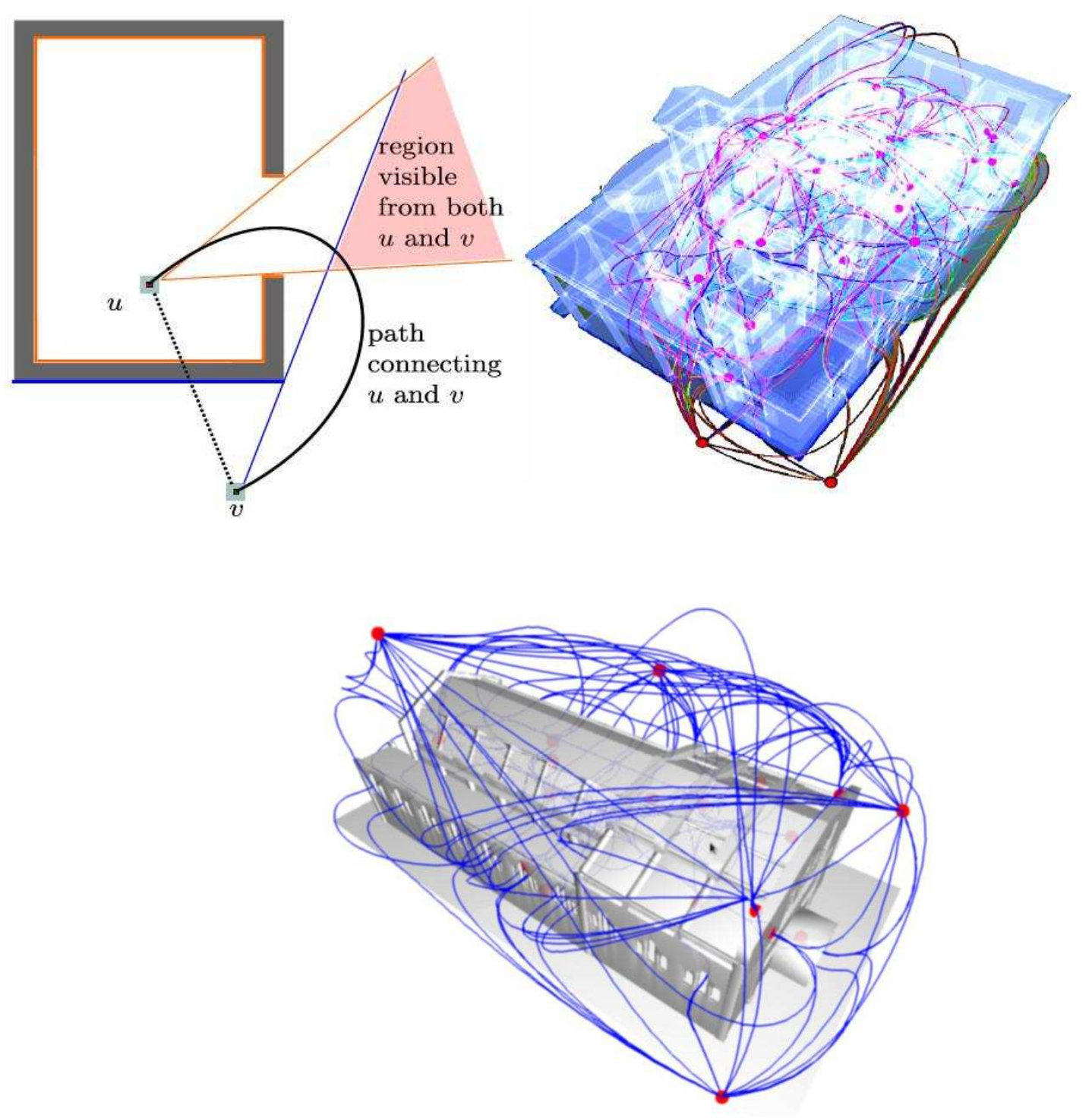


\section{Precomputation of probe images}

- Compute panoramic views for probes and frames of transition arcs

- Photorealistic rendering (using Blender 2.68a)

- panoramic views both for probes and transition arcs

- $1024^{\wedge} 2$ probe panoramas

- $256^{\wedge} 2$ transition video panoramas

Explore Map

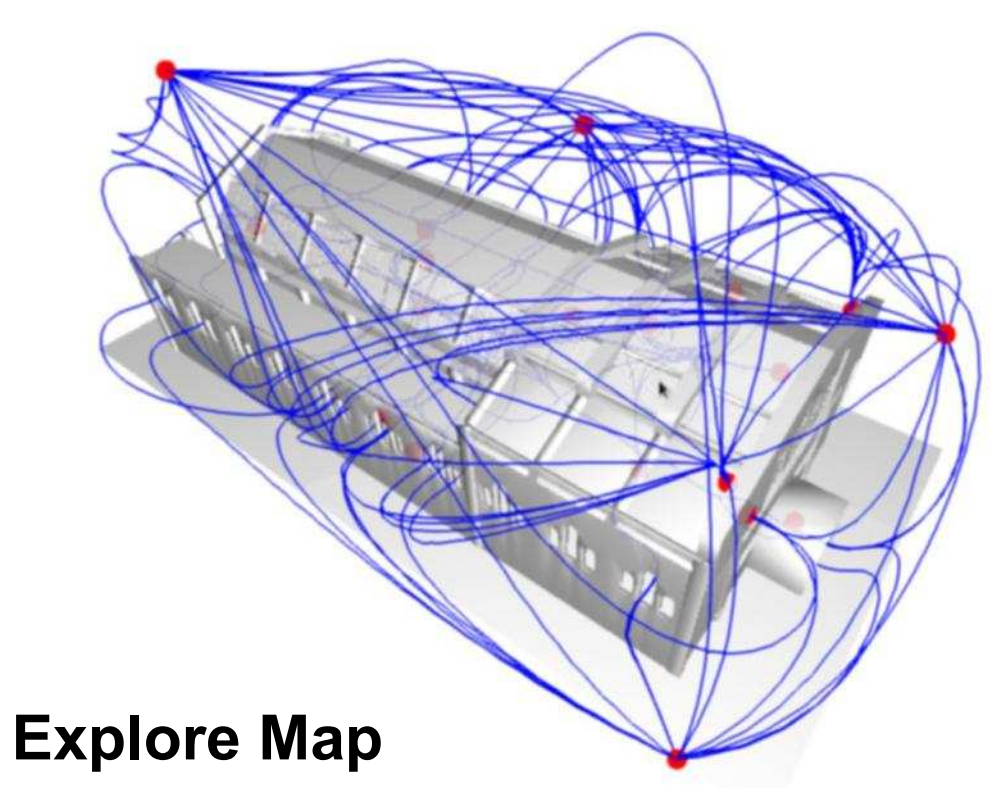

- 32 8-core PCs,

- Rendering times ranging from 40 minutes to 7 hours/model

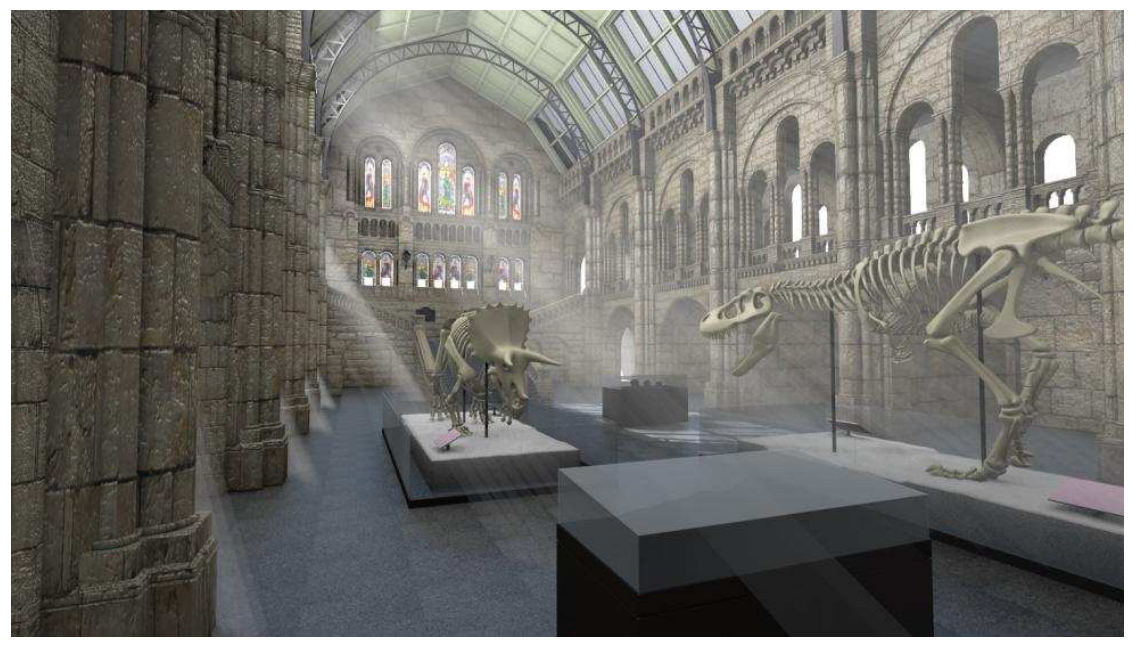




\section{Explore Maps - Processing Results}

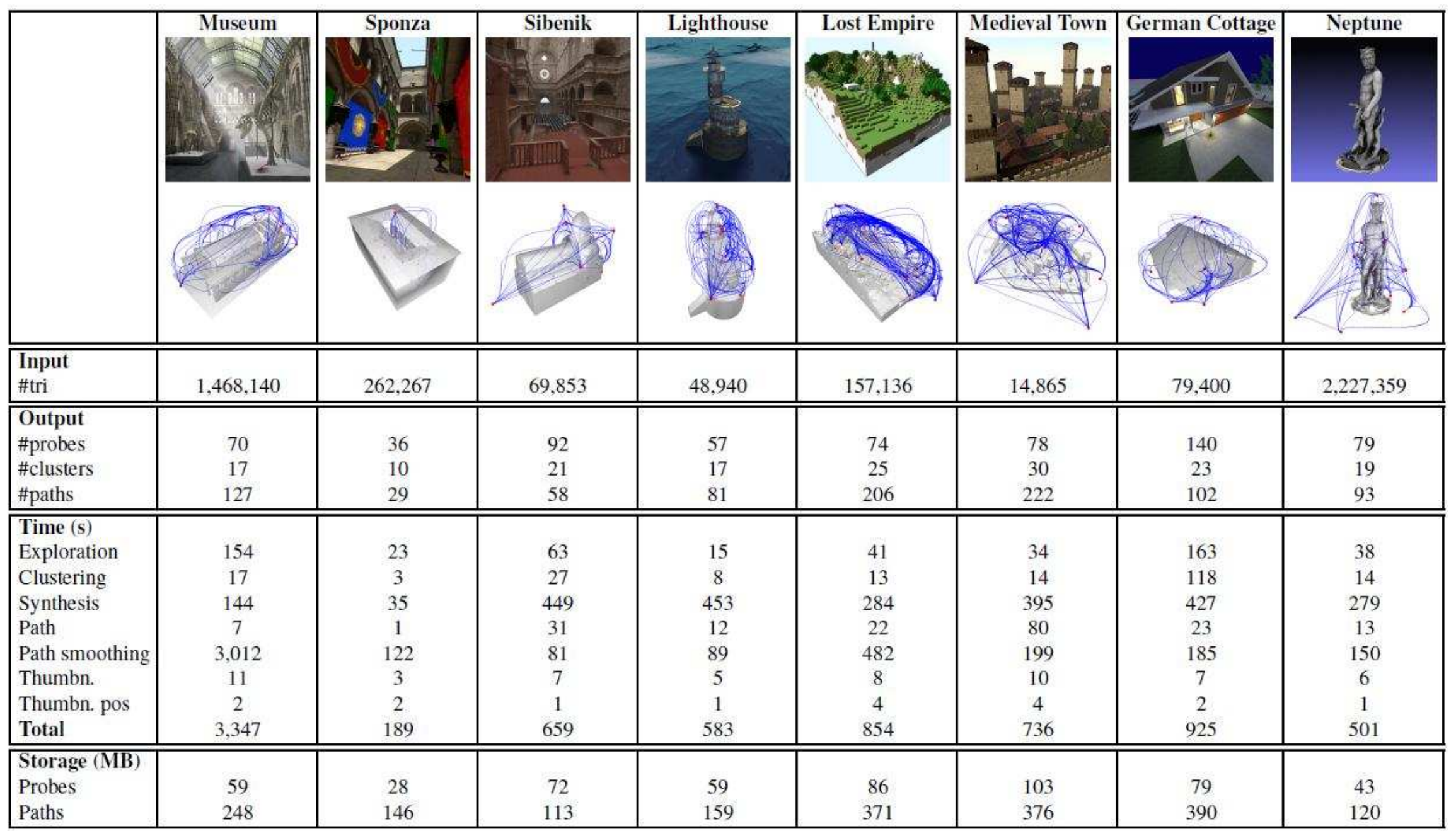




\section{Interactive Exploration}

- UI for Explore Maps

- WebGL implementation + JPEG + MP4

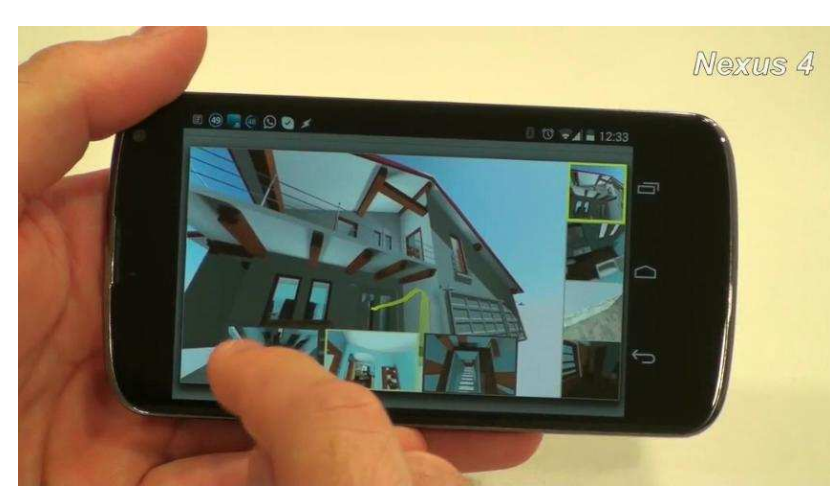

- Panoramic images: probes + transition path

- Closest probe selection

- Path alignment with current view

- Thumbnail goto

- Non-fixed orientation
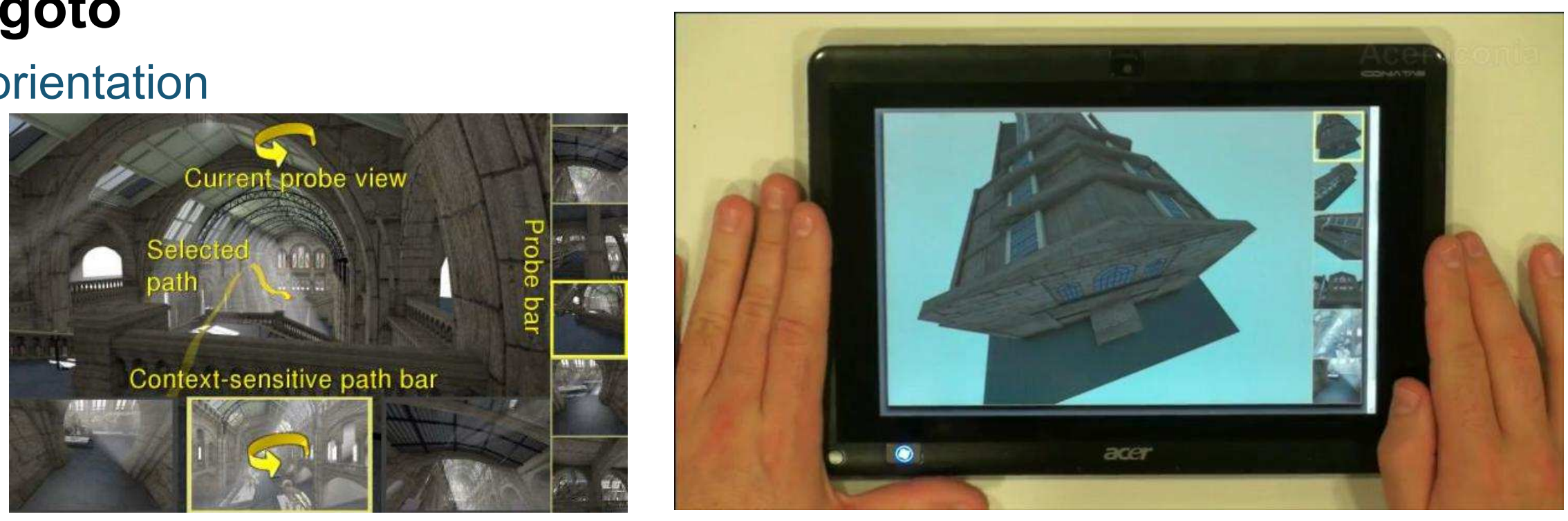


\section{Conclusion: Interactive Exploration}

- Interactive exploration of complex scenes

- Web/mobile enabled

- Pre-computed rendering

- state-of-the-art Global Illumination

- Graph-based navigation $\rightarrow$ guided exploration

- Limitations

- Constrained navigation

- Fixed set of camera positions

- Limited interaction

- Exploit panoramic views on paths $\rightarrow$ less constrained navigation

- Next part of the talk:

- A dynamic solution for complex illumination with smart computation 


\section{Part 4.5}

\section{Scalable Mobile Visualization:}

\section{Smart precomputation for complex lighting}

\section{Pere-Pau Vázquez, UPC}




\section{High quality illumination}

- Consistent illumination for AR

- Soft shadows

- Deferred shading

- Ambient Occlusion 


\section{Consistent illumination for AR}

- High-Quality Consistent Illumination in Mobile Augmented Reality by Radiance Convolution on the GPU [Kán, Unterguggenberger \& Kaufmann, 2015]

- Goal

- Achieve realistic (and consistent) illumination for synthetic objects in Augmented Reality environments 


\section{Consistent illumination for AR}

\section{- Overview}

- Capture the environment with the mobile

- Create an HDR environment map

- Convolve the HDR with the BRDF's of the materials

- Calculate radiance in realtime

- Add AO from an offline rendering as lightmaps

- Multiply with the AO from the synthetic object 


\section{Consistent illumination for AR}

- Capture the environment with the mobile

- Rotational motion of the mobile

- In yaw and pitch angles to cover all sphere directions

- Images accumulated to a spherical environment map

- HDR environment map constructed while scanning

- Projecting each camera image

- According to the orientation and inertial measurement of the mobile

- Low dynamic range imaging is transformed to HDR

- Camera uses auto-exposure

- Two overlapping images will have slightly different exposure

- Alignment correction based on feature matching

- All in the device 


\section{Consistent illumination for AR}

- Convolve the HDR with the BRDF's of the materials

- Use MRT to support several convolutions at once

- Assume distant light

- One single light reflection on the surface

- Scene materials assumed non-emissive

- Use a simplified rendering equation

- Weight with AO (obtained offline)

- Built for real and synthetic objects

- Nee the geometry of the scene

- Use a proxy geometry for the objects of the real world

- Cannot be simply done on the fly 


\section{Consistent illumination for AR}

- Results

Without AO

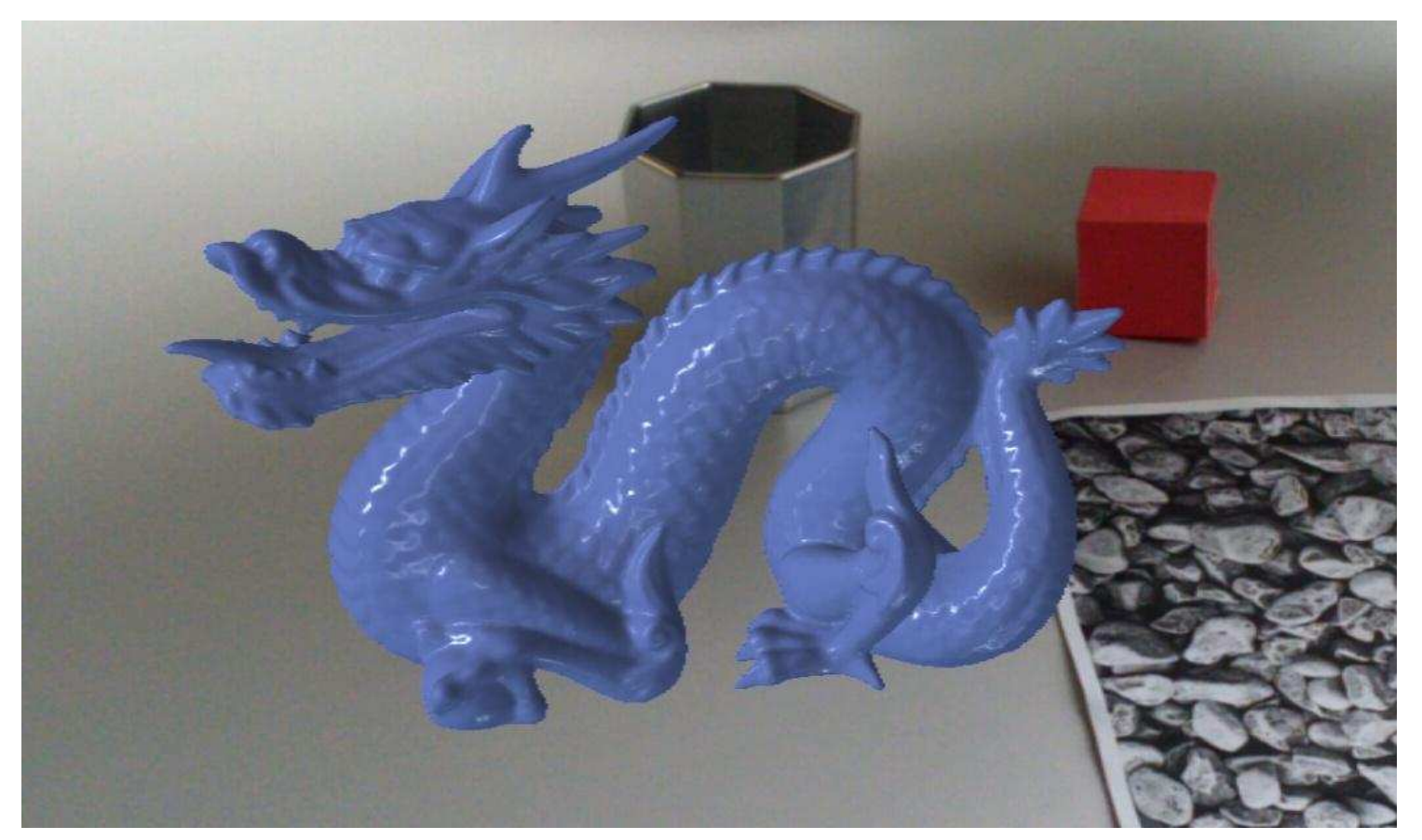

With AO

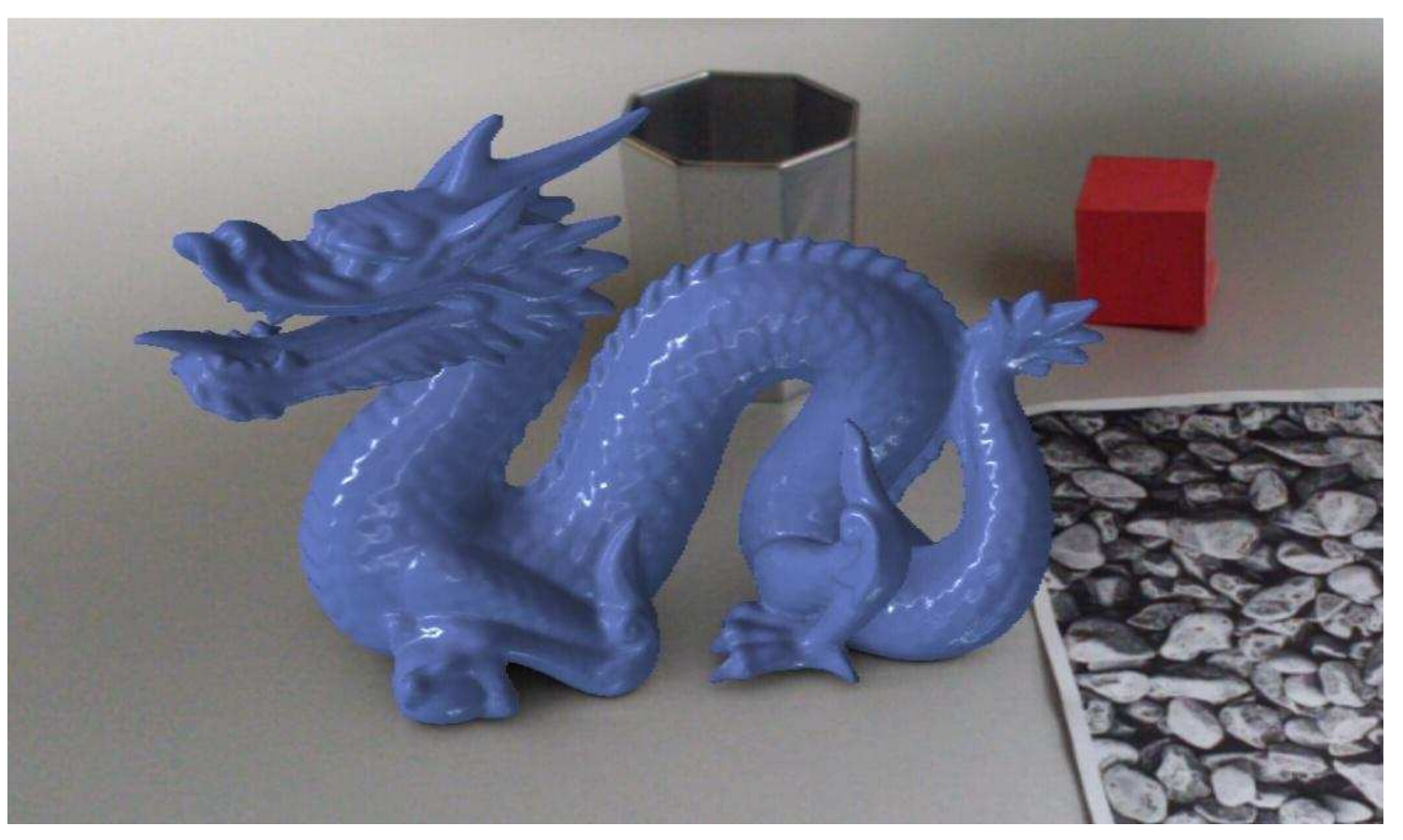

Images courtesy of Peter Kán 


\section{Consistent illumination for AR}

- Performance

\begin{tabular}{|l|r|r|}
\hline 3D model & \# triangles & Framerate \\
\hline Reflective cup & $25.6 \mathrm{~K}$ & $29 \mathrm{fps}$ \\
\hline Teapot & $15.7 \mathrm{~K}$ & $30 \mathrm{fps}$ \\
\hline Dragon & $229 \mathrm{~K}$ & $13 \mathrm{fps}$ \\
\hline
\end{tabular}

- Limitations

- Materials represented by Phong BRDF

- AO and most shading (e.g. reflection maps) is baked 


\section{Soft shadows using cubemaps}

- Efficient Soft Shadows Based on Static Local Cubemap [Bala \& Lopez Mendez, 2016]

- Goal

- Soft shadows in realtime
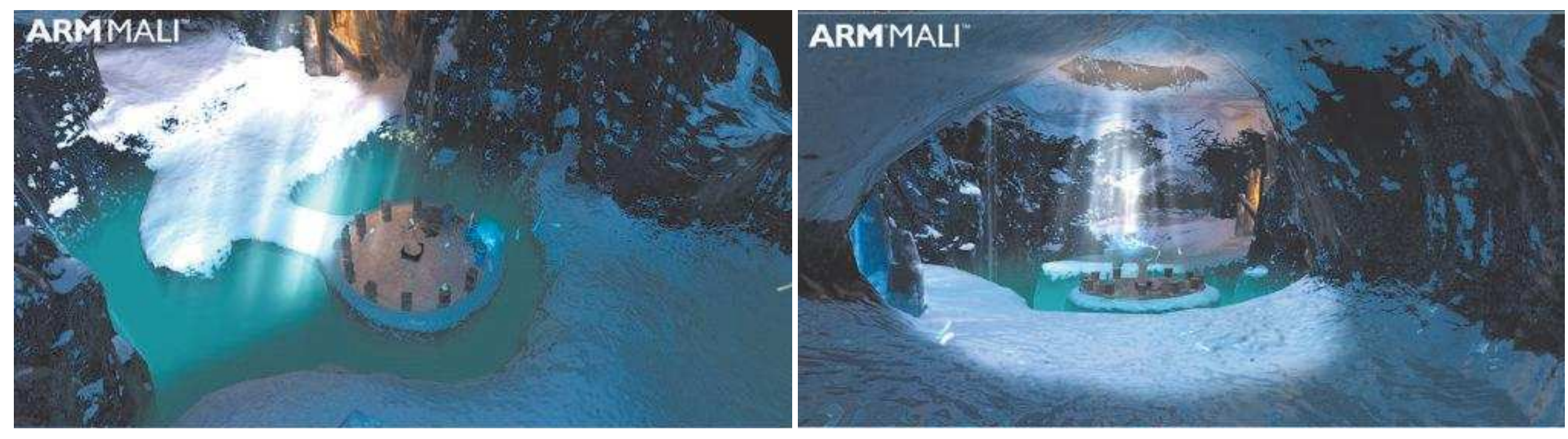

Taken from https://community.arm.com/graphics/b/blog/posts/dynamic-soft-shadows-based-on-local-cubemap 


\section{Soft shadows using cubemaps}

\section{- Overview}

- Create a local cube map

- Offline recommended

- Stores color and transparency of the environment

- Position and bounding box

- Approximates the geometry

- Local correction

- Using proxy geometry

- Apply shadows in the fragment shader 


\section{Soft shadows using cubemaps}

- Generating shadows

- Fetch texel from cubemap

- Using the fragment-to-light vector

- Correct the vector before fetching

- Using the scene geometry (bbox) and cubemap creation position

"To provide the equivalent shadow rays

- Apply shadow based on the alpha value

- Soften shadow

- Using mipmapping and addressing according to the distance 


\section{Soft shadows using cubemaps}

\section{- Conclusions}

- Does not need to render to texture

- Cubemaps must be pre-calculated

- Requires reading multiple times from textures

- Stable

- Because cubemap does not change

\section{- Limitations}

- Static, since info is precomputed 


\section{Physically-based Deferred Rendering}

- Physically Based Deferred Shading on Mobile [Vaughan Smith \& Einig, 2016]

- Goal:

- Adapt deferred shading pipeline to mobile

- Bandwidth friendly

- Using Framebuffer Fetch extension

- Avoids copying to main memory in OpenGL ES 


\section{Physically-based Deferred Rendering}

\section{- Overview}

- Typical deferred shading pipeline

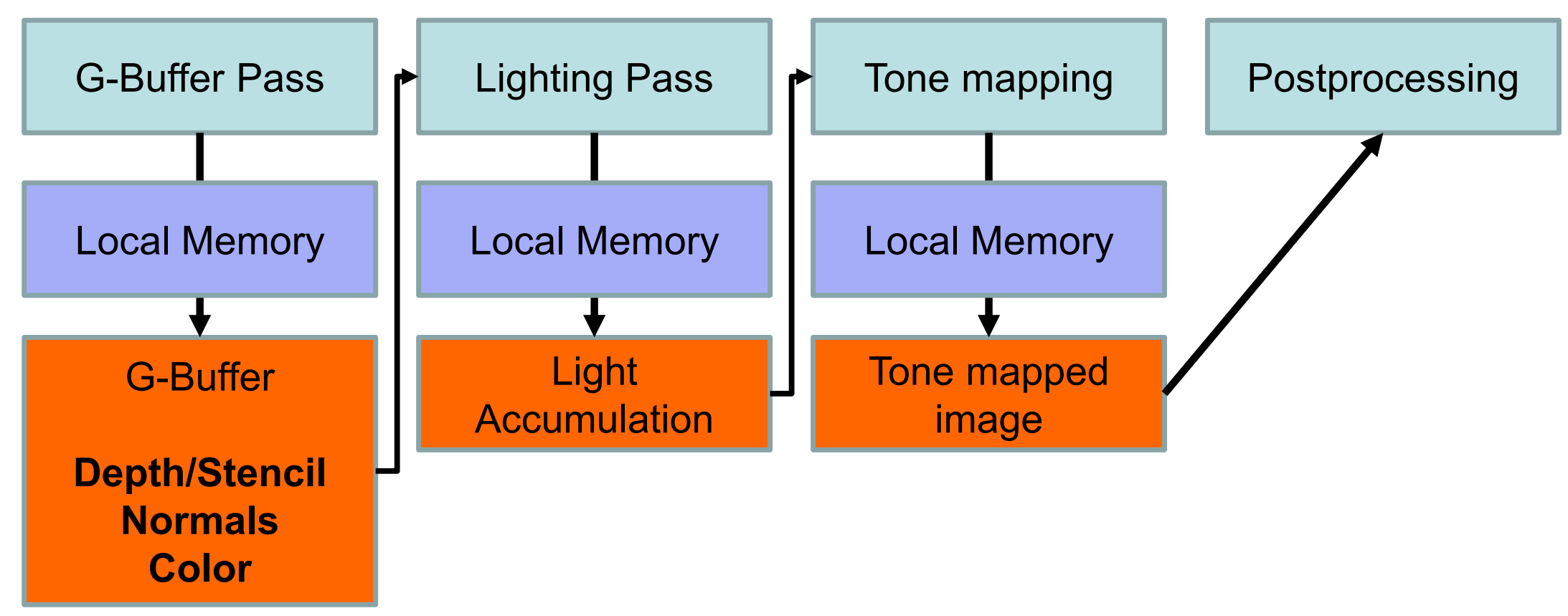




\section{Physically-based Deferred Rendering}

- Main idea: group G-buffer, lighting \& tone mapping into one step

- Further improve by using Pixel Local Storage extension

- G-buffer data is not written to main memory

- Usable when multiple shader invocations cover the same pixel

- Resulting pipeline reduces bandwidth

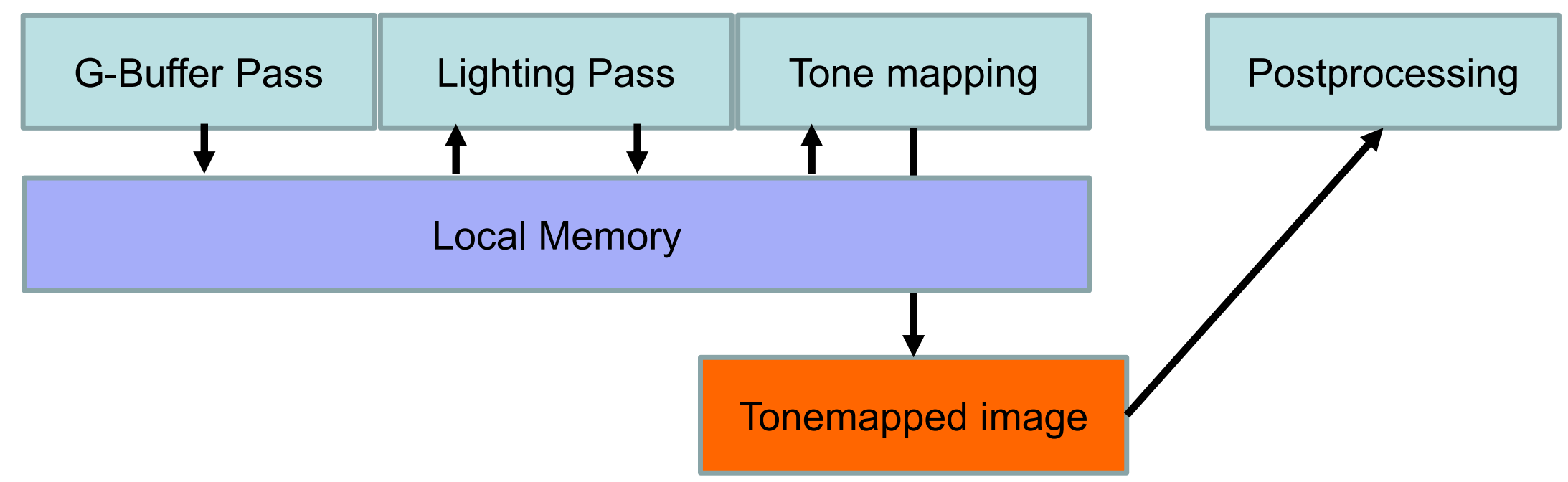




\section{Physically-based Deferred Rendering}

- Two G-buffer layouts proposed

- Specular G-buffer setup (160 bits)

- Rgb10a2 highp vec4 light accumulation

- R32f highp float depth

- 3 x rgba8 highp vec4: normal, base color \& specular color

- Metallicness G-buffer setup (128 bits, more bandwidth efficient)

- Rgb10a2 highp vec4 light accumulation

- R32f highp float depth

- 2 x rgba8 highp vec4: normal \& roughness, albedo or reflectance metallicness 


\section{Physically-based Deferred Rendering}

\section{- Lighting}

- Use precomputed HDR lightmaps to represent static diffuse lighting

- Shadows \& radiosity

- Can be compressed with ASTC (supports HDR data)

- PVRTC, RGBM can also be used for non HDR formats

- Geometry pass calculates diffuse lighting

- Specular is calculated using Schlick's approximation of Fresnel factor 


\section{Physically-based Deferred Rendering}

- Results (PowerVR SDK)

- Fewer rendering tasks

- meaning that the G-buffer generation, lighting, and tonemapping stages are properly merged into one task.

- reduction in memory bandwidth

- $53 \%$ decrease in reads and a $54 \%$ decrease in writes

\section{- Limitations}

- Still not big frame rates 


\section{Ambient Occlusion in mobile}

- Optimized Screen-Space Ambient Occlusion in Mobile Devices [Sunet \& Vázquez, Web3D 2016]

- Goal: Study feasibility of real time AO in mobile

- Analyze most popular AO algorithms: Crytek's, Alchemy's, Nvidia's Horizon-Based AO (HBAO), and Starcraft II (SC2)

- Evaluate their AO pipelines step by step

- Design architectural improvements

- Implement and compare 


\section{Ambient Occlusion in mobile}

- Ambient Occlusion. Simplification of rendering equation

- The surface is a perfect diffuse surface (BRDF constant)

- Light potentially reaches a point $p$ equally in all directions

- But takes into account point's visibility

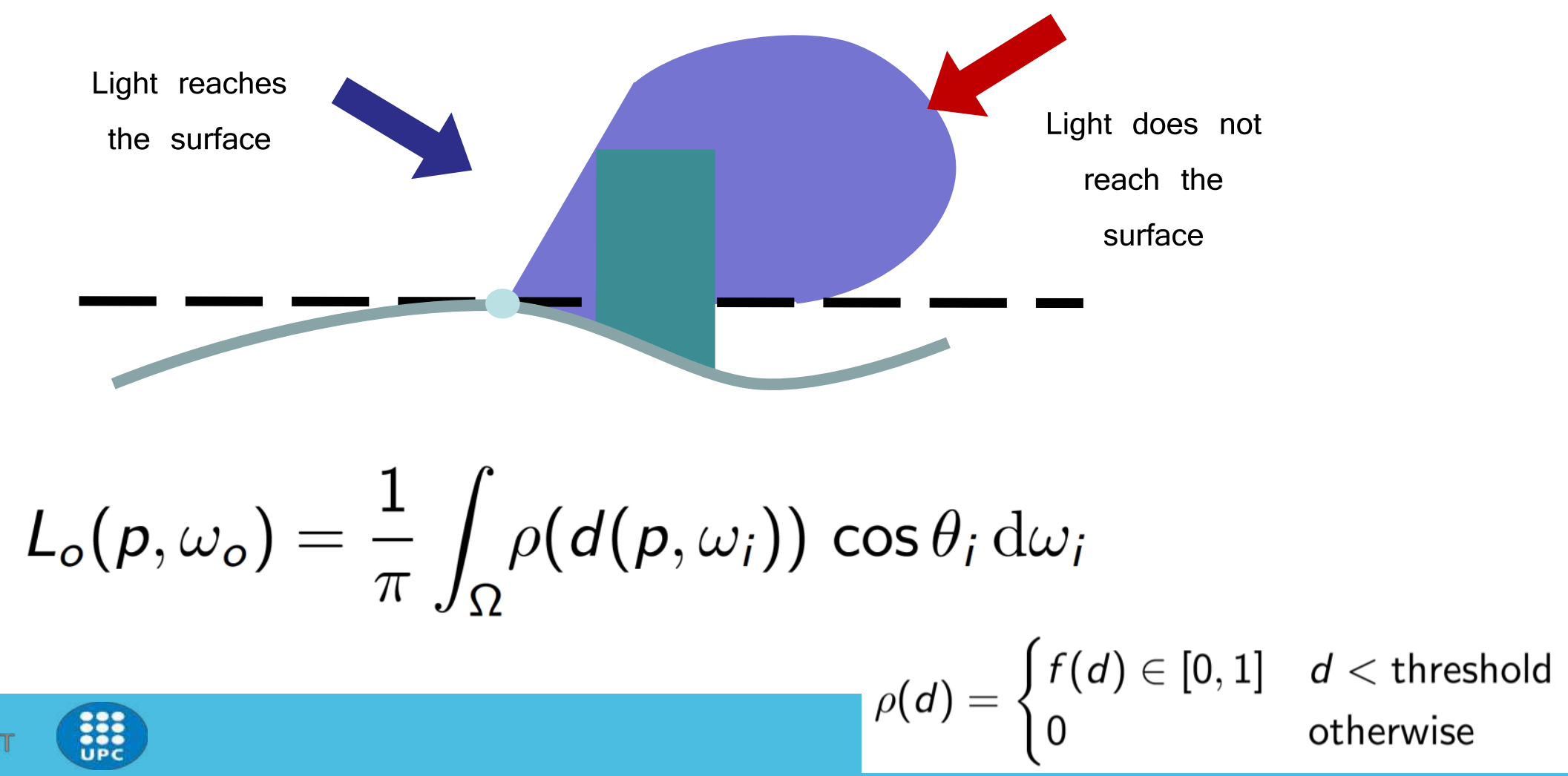




\section{Ambient Occlusion in mobile}

\section{- AO typical implementations}

- Precomputed AO: Fast \& high quality, but static, memory hungry

- Ray-based: High quality, but costly, visible patterns...

- Geometry-based: Fast w/ proxy structures, but lower quality, artifacts/noise...

- Volume-based: High quality, view independent, but costly

- Screen-space:

- Extremely fast

- View-dependent

- [mostly] requires blurring for noise reduction

- Very popular in video games (e.g. Crysis, Starcraft 2, Battlefield 3...) 


\section{Ambient Occlusion in mobile}

\section{- Screen-space AO:}

- Approximation to AO implemented as a screen-space post-processing

- ND-buffer provides coarse approximation of scene's geometry

- Sample ND-buffer to approximate (estimate) ambient occlusion instead of shooting rays
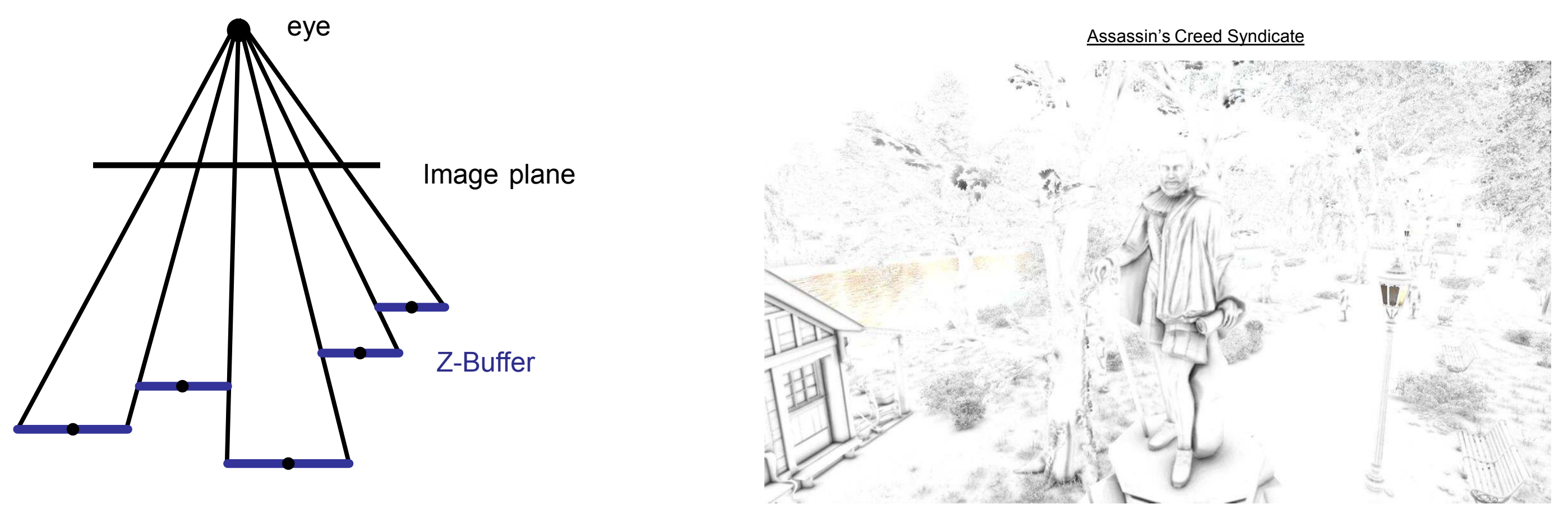


\section{Ambient Occlusion in mobile}

\section{- SSAO pipeline}

1. Generate ND (normal + depth, OpenGL ES 2) or G-Buffer (ND + RGB..., OpenGL ES 3.+)

2. Calculate $A O$ factor for visible pixels

a. Generate a set of samples of positions/vectors around the pixel to shade.

b. Get the geometry shape (position/normal...)

c. Calculate $\mathrm{AO}$ factor by analyzing shape...

3. Blur the AO texture to remove noise artifacts

4. Final compositing 


\section{Ambient Occlusion in mobile}

\section{- Optimizations. G-Buffer storage}

- G-Buffer with less precision $(32,16,8)$

- 8 not enough

- 16 and 32 similar quality

- Normal storage (RGB vs RG)

- RGB normals are faster
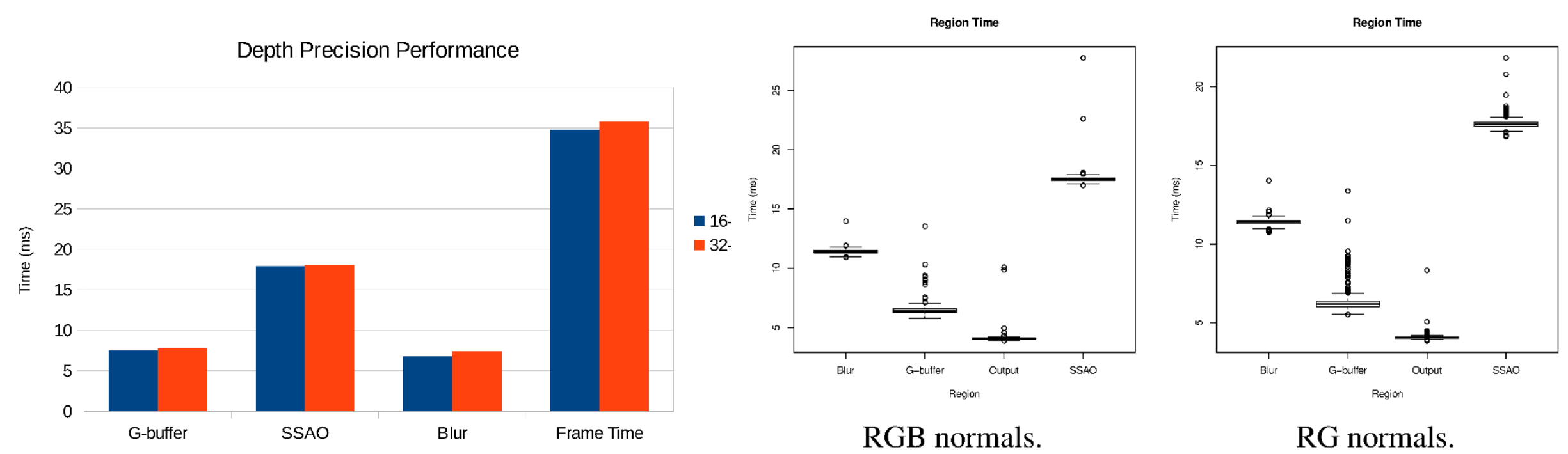


\section{Ambient Occlusion in mobile}

- Optimizations. Sampling

- AO samples generation (disc and hemisphere)

- Desktops use up to 32

- With mobile, 8 is the affordable amount

- Pseudo-random samples produces noticeable patterns

- Our proposed solution

- Compute sampling patterns offline

- 2D: 8-point Poisson disc

- 3D: 8-point cosine-weighted hemisphere (Malley's approach, as in [Pharr and Humprheys, 2010])

- Scaling and rotating the resulting pattern ([Chapman, 2011])

- Predictable, reproducible, robust 


\section{Ambient Occlusion in mobile}

- Optimizations. Getting geometry positions

- Transform samples to 3D

- Inverse transform vs similar triangles

- Precision for speed

- Similar triangles are faster

- Storing depth vs storing 3D positions in G-Buffer

- Trades bandwidth for memory

- Depth slightly better

- Better profile for the application

Frame Time
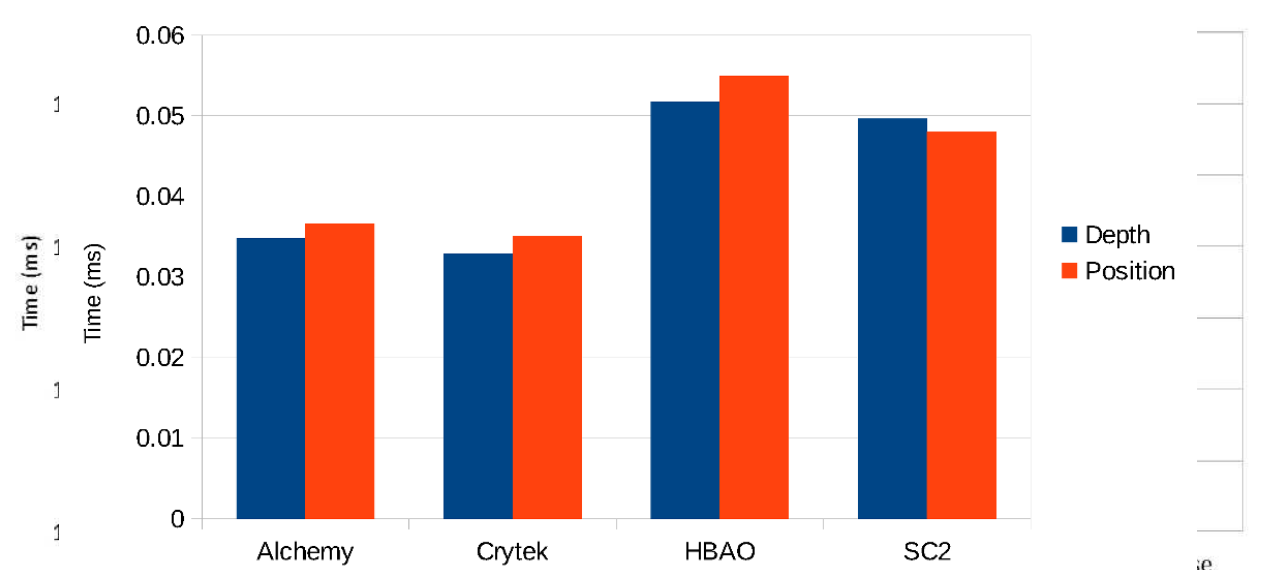


\section{Ambient Occlusion in mobile}

- Optimizations. Banding \& Noise

- Fixed sampling pattern produces banding (left)

- Random sampling reduces banding but adds noise (middle)

- SSAO output is typically blurred to remove noise (right)

- But blurs edges
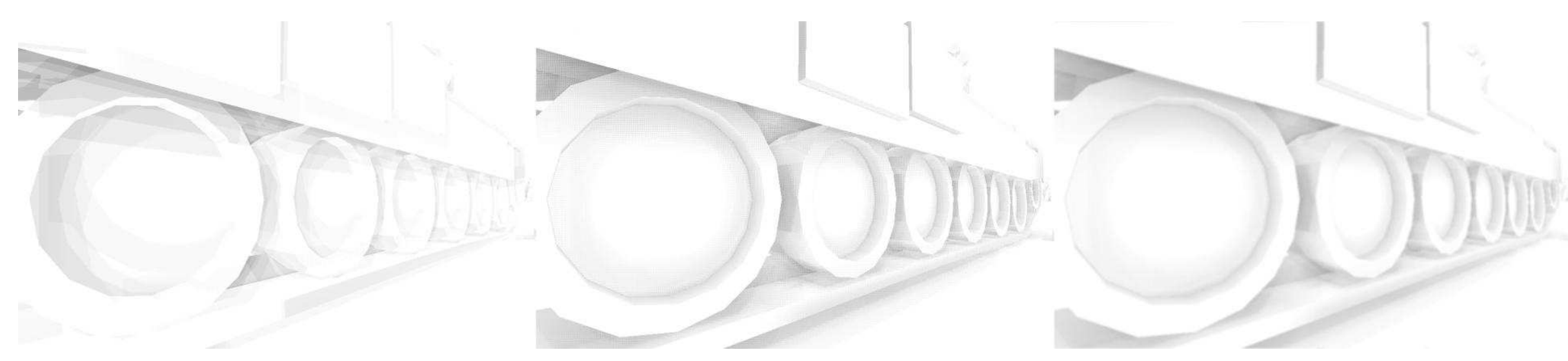


\section{Ambient Occlusion in mobile}

- Optimizations. Banding \& Noise

- User bilateral filter instead

- Works better

- Improve timings with separable filter
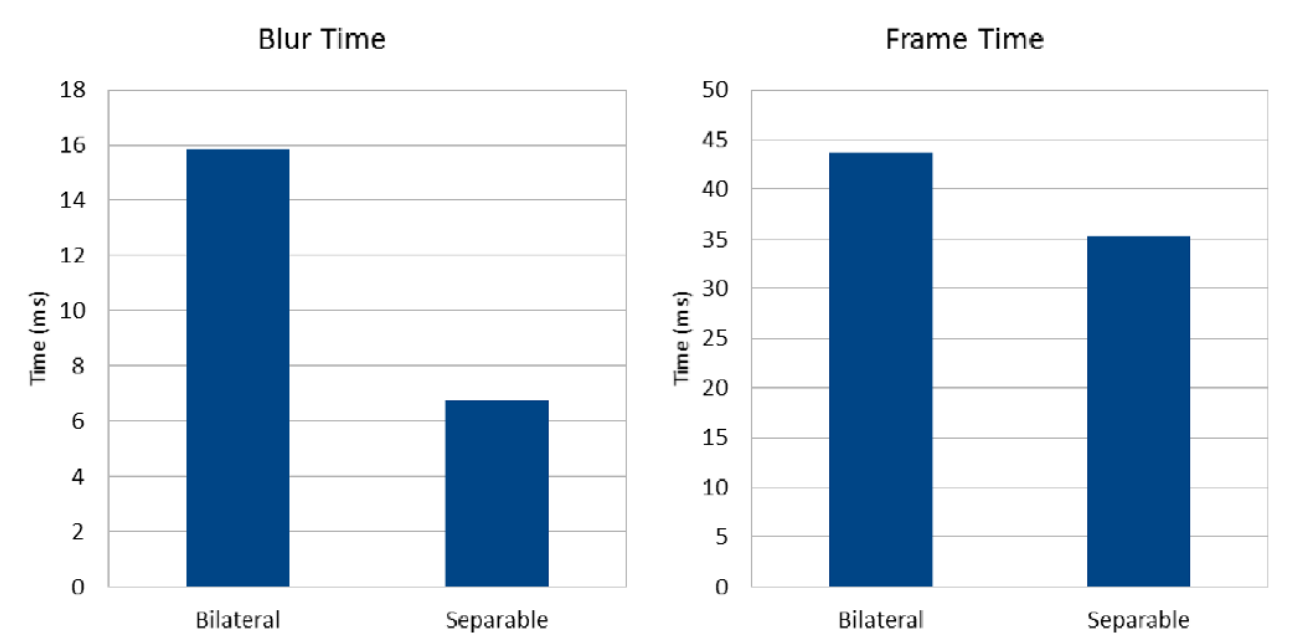

$$
\begin{gathered}
B F[]_{p}=\frac{1}{W_{p}} \sum_{q \in S} G_{\sigma_{s}}(|| p-q||) G_{\sigma_{r}}\left(\left|I_{q}-I_{p}\right|\right) I_{q} \\
G_{\sigma}(x)=\frac{1}{\sigma \sqrt{2 \pi}} \exp \left(-\frac{x^{2}}{2 \sigma^{2}}\right)
\end{gathered}
$$




\section{Ambient Occlusion in mobile}

- Optimizations. Progressive AO

- Amortize AO throughout many frames

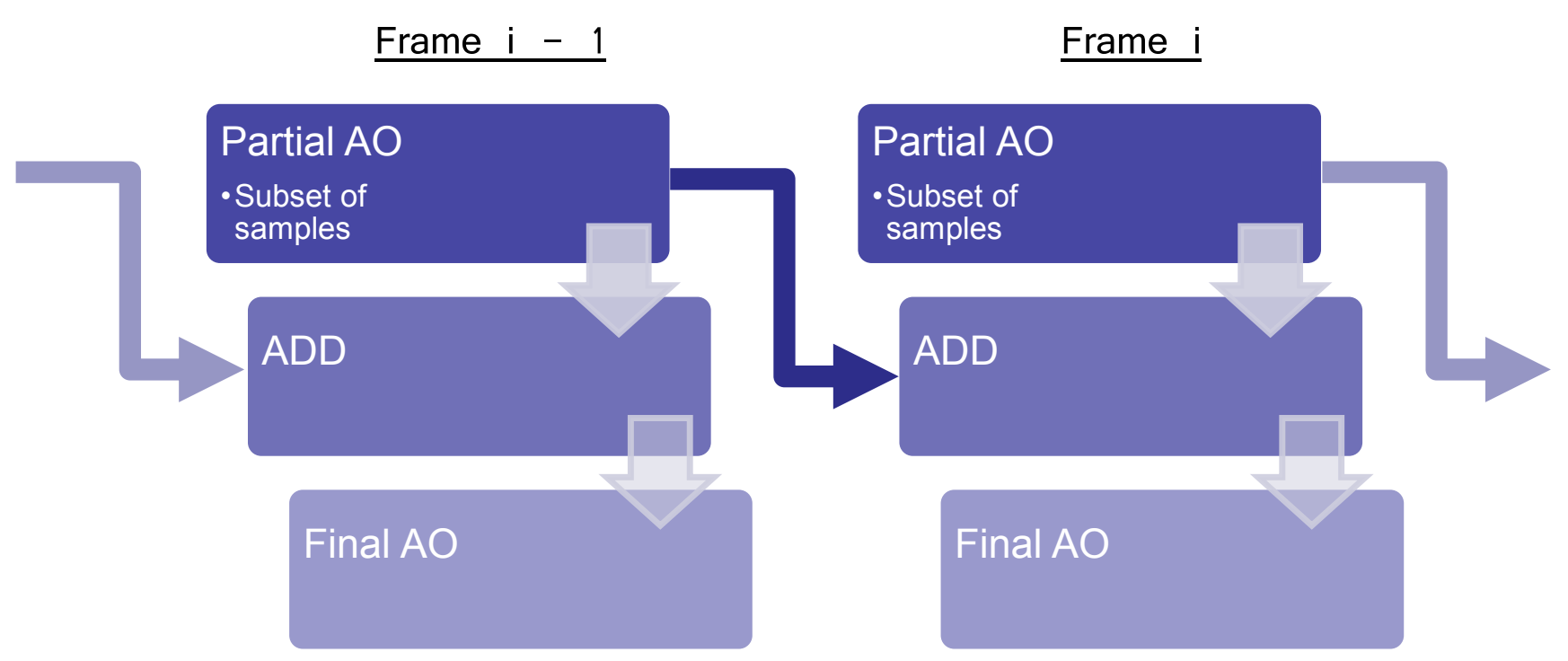




\section{Ambient Occlusion in mobile}

\section{- Optimizations}

- Naïve improvement: Reduce the calculation to a portion of the screen

- Mobile devices have a high PPI resolution

- Reduction improves timings dramatically while keeping high quality

- Typical reduction:

- Offscreen render to $1 / 4^{\text {th }}$ of the screen

- Scale-up to fill the screen 


\section{Ambient Occlusion in mobile}

- Results

\begin{tabular}{|l|c|c|}
\hline Algorithm & $\begin{array}{l}\text { Optimized (not } \\
\text { progressive) }\end{array}$ & $\begin{array}{l}\text { Optimized + } \\
\text { progressive }\end{array}$ \\
\hline Starcraft 2 & $17.8 \%$ & $38.5 \%$ \\
\hline HBAO & $25.6 \%$ & $39.2 \%$ \\
\hline Crytek & $23.4 \%$ & $35.0 \%$ \\
\hline Alchemy & $24.8 \%$ & $38.2 \%$ \\
\hline
\end{tabular}




\section{Ambient Occlusion in mobile}

\section{- Conclusions}

- Developed an optimized pipeline for mobile AO

- Analyzed the most popular AO techniques

- Improved several important steps of the pipeline

- Proposed some extra contributions (e.g. progressive AO)

- Achieved realtime framerates with high quality

- Developed techniques can be used in WebGL

- Future Work

- Further improvement of the pipeline

- Developing "Homebrew" method

- With all known improvements

- Some extra tricks

- Not ready for prime time yet 


\section{Part 4.5}

\section{Scalable Mobile Visualization: Volumetric Data}

Pere-Pau Vázquez, UPC 


\section{Rendering Volumetric Datasets}

- Introduction

- Challenges

- Architectures

- GPU-based ray casting on mobile

- Conclusions 


\section{Rendering Volumetric Datasets}

Capturing

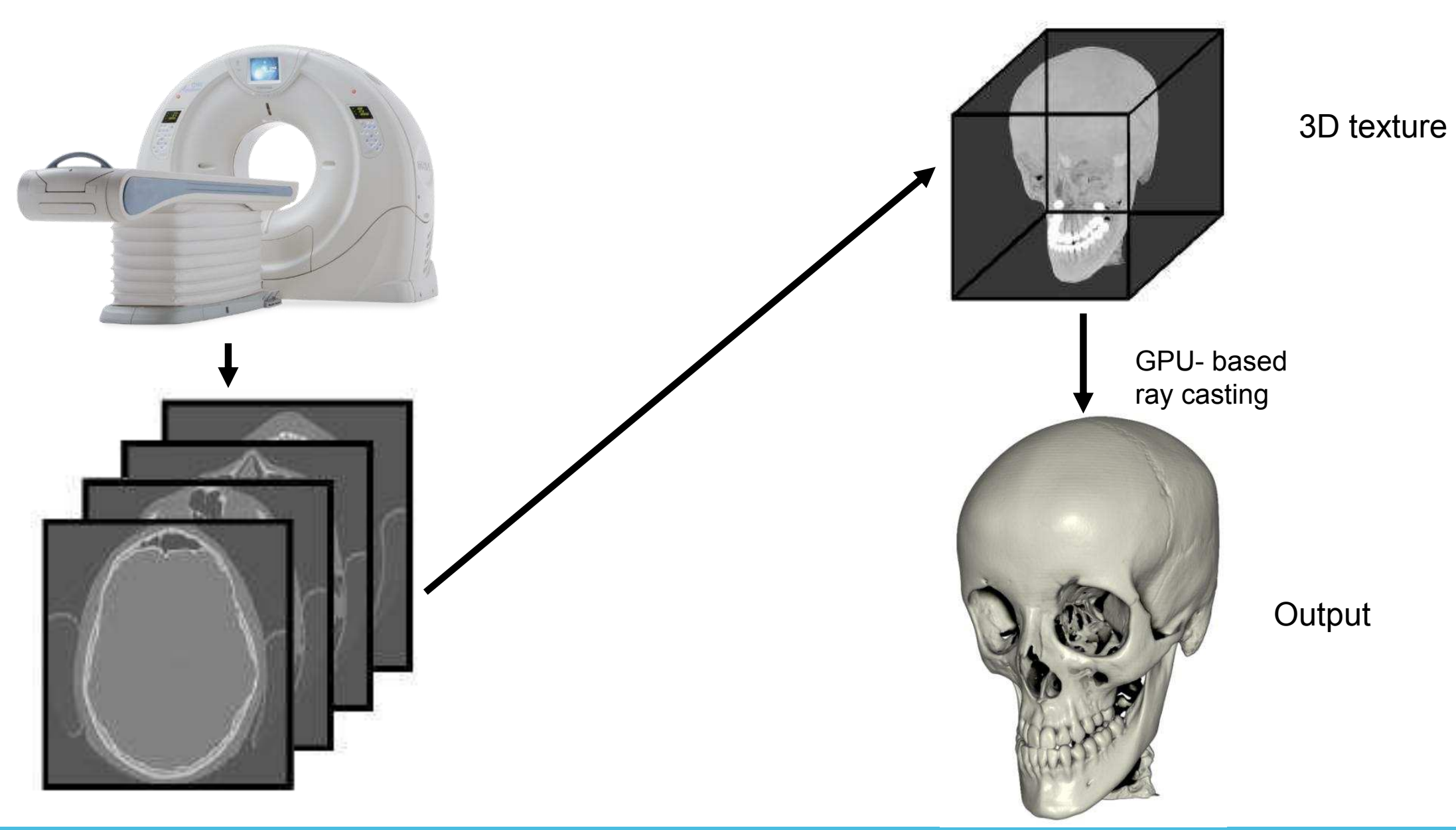




\section{Rendering Volumetric Datasets}

\section{- Introduction}

- Volume datasets

- Sizes continuously growing (e.g. $\left.>1024^{3}\right)$

- Complex data (e.g. 4D)

- Rendering algorithms

- GPU intensive

- State-of-the-art is ray casting on the fragment shader

- Interaction

- Edition, inspection, analysis, require a set of complex manipulation techniques 


\section{Rendering Volumetric Datasets}

- Desktop vs mobile

- Desktop rendering

- Large models on the fly

- Huge models with the aid of compression/multiresolution schemes

- Mobile rendering

- Standard sizes (e.g. $512^{3}$ ) still too much for the mobile GPUs

- Rendering algorithms GPU intensive

- State-of-the-art is GPU-based ray casting

- Interaction is difficult on a small screen

- Changing TF, inspecting the model... 


\section{Rendering Volumetric Datasets}

- Challenges on mobile:

- Memory:

- Model does not fit into memory

- Use client server approach / compress data

- GPU capabilities:

- Cannot use state of the art algorithm (e.g. no 3D textures)

- Texture arrays

- GPU horsepower:

- GPU unable to perform interactively

- Progressive rendering methods

- Small screen

- Not enough details, difficult interaction 


\section{Rendering Volumetric Datasets}

- Mobile architectures

- Server-based rendering

- Hybrid approaches

- Pure mobile rendering

- Server-based and hybrid rely on high bandwidth communication 


\section{Rendering Volumetric Datasets}

- Pure mobile rendering

- Move all the work to the mobile

- Nowadays feasible

- Direct Volume Rendering on mobile. Algorithms

- Slices

- 2D texture arrays

- 3D textures 


\section{Rendering Volumetric Datasets}

Axis-aligned
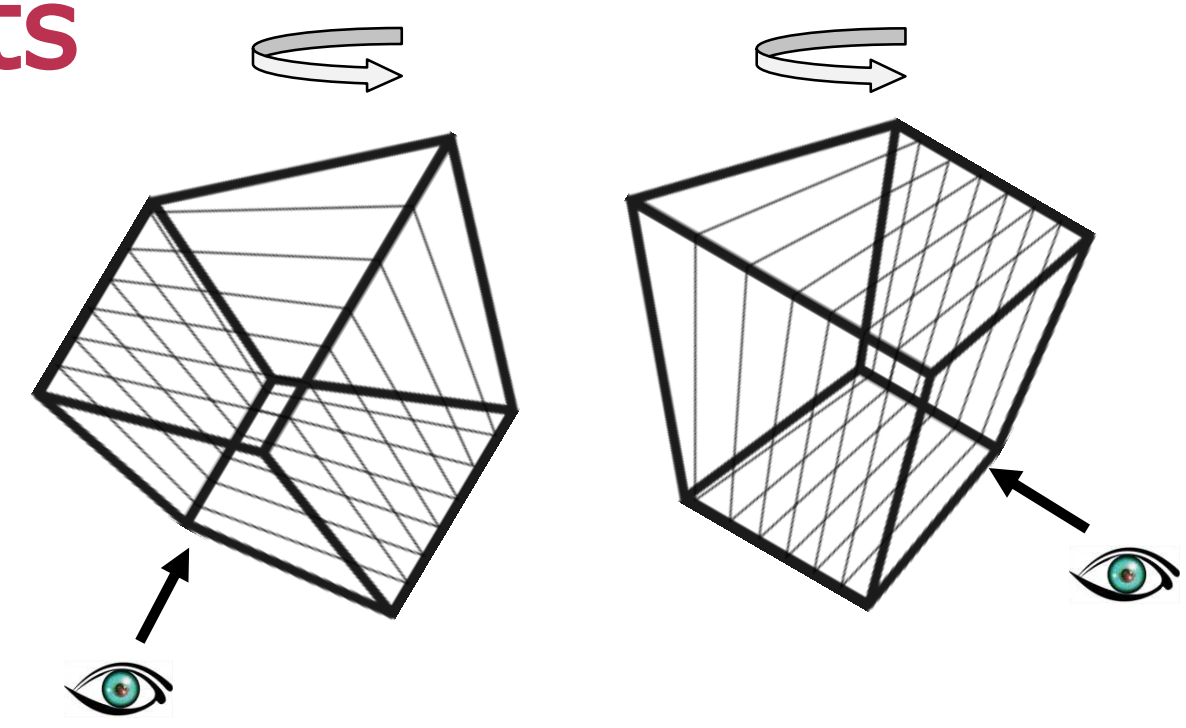

- Improvement: Oblique slices [Kruger 2010]

View-aligned

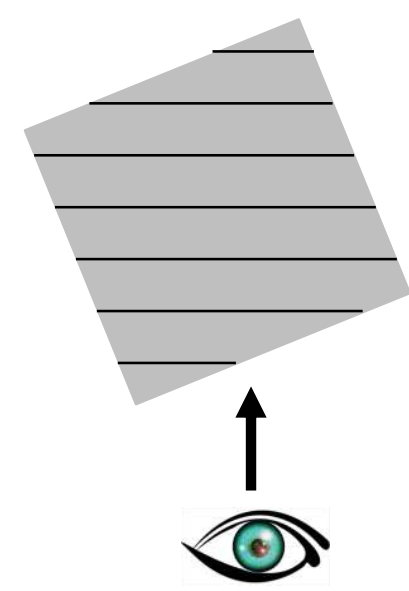

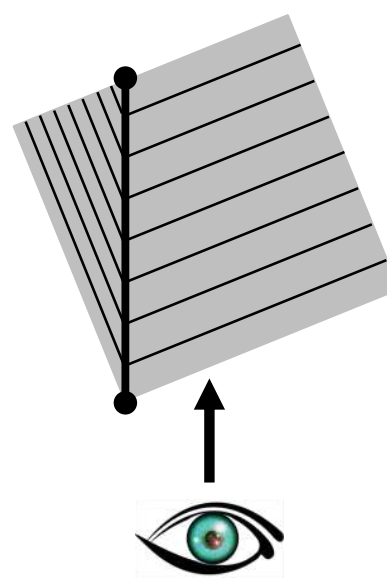

Oblique 


\section{Rendering Volumetric Datasets}

- 2D texture arrays + texture atlas [Noguera et al. 2012]

- Simulate a 3D texture using an array of 2D textures

- Implement GPU-based ray casting

- High quality

- Relatively large models

- Costly

- Cannot use hardware trilinear interpolation 


\section{Rendering Volumetric Datasets}

3D texture representation

\section{- 2D texture arrays + texture atlas}
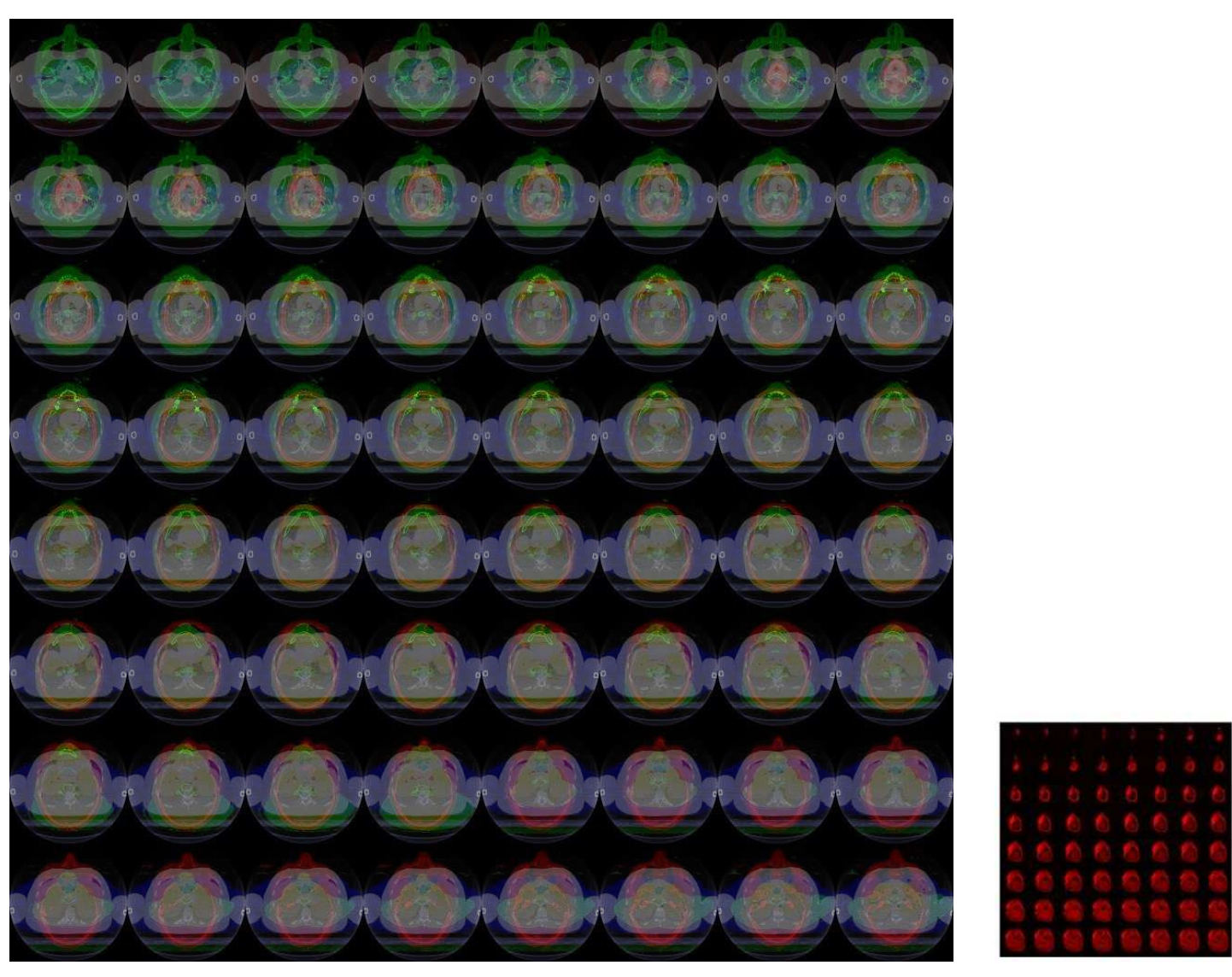

Texture mosaic representation

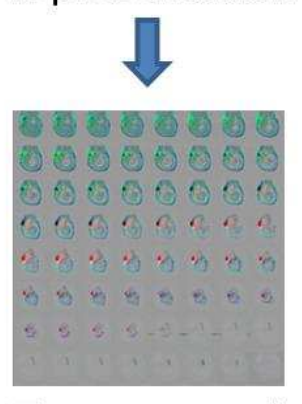

Texture mosaic per channel Illustration !

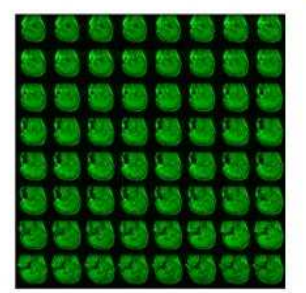

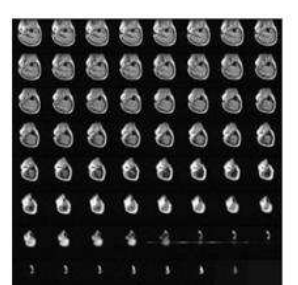




\section{Rendering Volumetric Datasets}

- 2D texture arrays + compression [Valencia \& Vázquez, 2013]

- Increase the supported sizes

- Increase framerates

\begin{tabular}{|c|c|c|c|c|c|c|}
\hline $\begin{array}{c}\text { Compression } \\
\text { format }\end{array}$ & $\begin{array}{c}\text { Compression } \\
\text { ratio }\end{array}$ & $\begin{array}{c}\text { RBA } \\
\text { format }\end{array}$ & $\begin{array}{c}\text { RGBA } \\
\text { format }\end{array}$ & $\begin{array}{c}\text { GPU } \\
\text { support }\end{array}$ & $\begin{array}{c}\text { Overall } \\
\text { performance }\end{array}$ & $\begin{array}{c}\text { Overall } \\
\text { quality }\end{array}$ \\
\hline ETC1 & $4: 1$ & Yes & No & All GPUs & Good (RC) & Good \\
\hline PVRTC & $8: 1$ and $16: 1$ & Yes & Yes & PowerVR & Not so good & Bad \\
\hline ATITC & $4: 1$ & Yes & Yes & Adreno & Good (RC) & Good \\
\hline
\end{tabular}




\section{Rendering Volumetric Datasets}

\section{- 2D texture arrays + compression}

- ATITC: improves performance from $6 \%$ to $19 \%$. With an average of $13.1 \%$ and a low variance of performance.

- ETC1(-P): improves performance from $6.3 \%$ to $69.5 \%$. With an average of $32.6 \%$ and the highest variance of performance.

- PVRTC-4BPP: improves performance from 4.7\% and 36.\% and PVRTC-2BPP: from 9,5\% to $36,5 \%$. The average performance of both methods is $\sim 15 \%$ with high variance. 


\section{Rendering Volumetric Datasets}

\section{- 2D texture arrays + compression}

- Ray-casting: gain performance in average of $33 \%$.

- Slice-based: gain performance in average of $8 \%$.

- Ray-casting frame rates are better in all cases compared to slice-based. 


\section{Rendering Volumetric Datasets}

- 2D texture arrays + compression

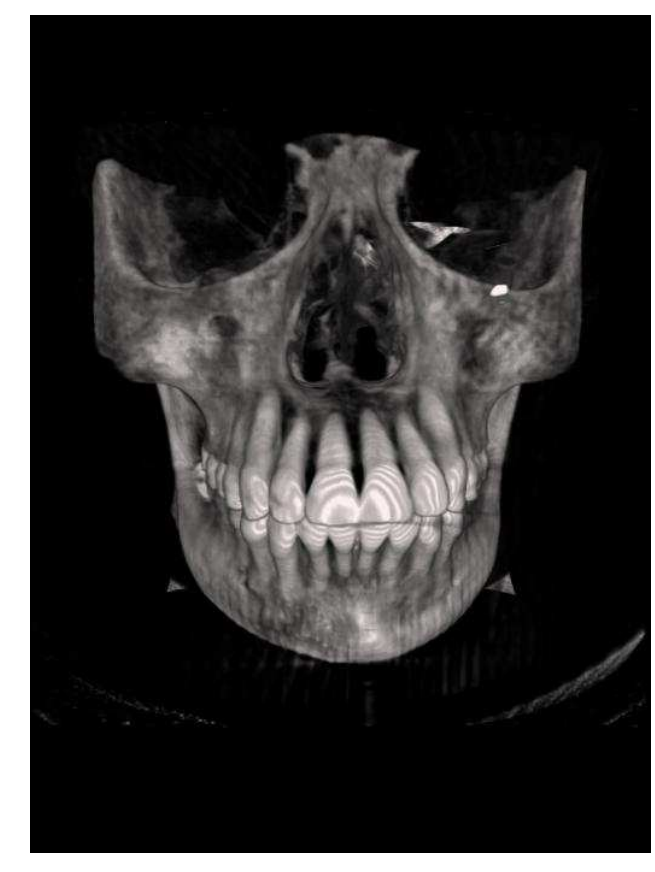

Uncompressed

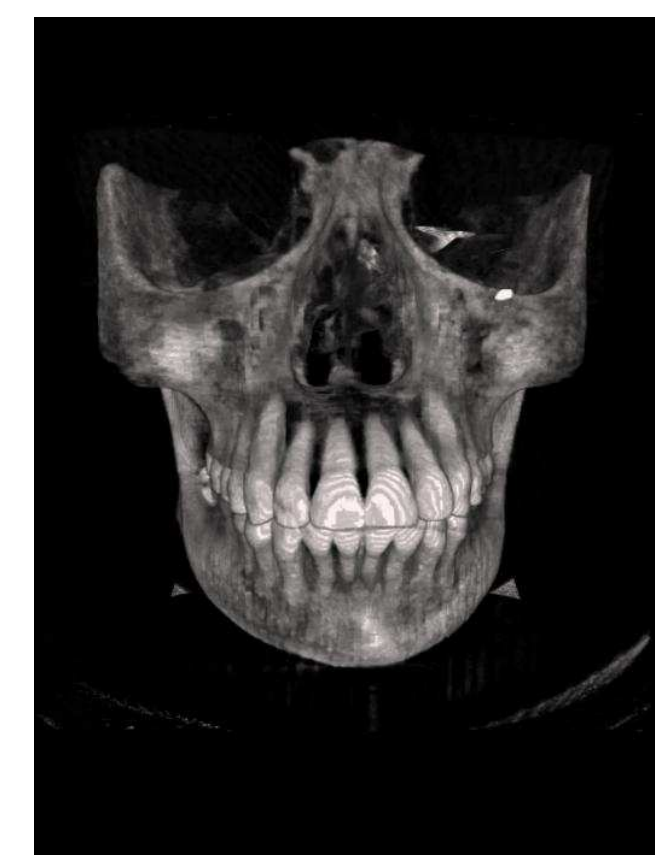

Compressed with ATI-I

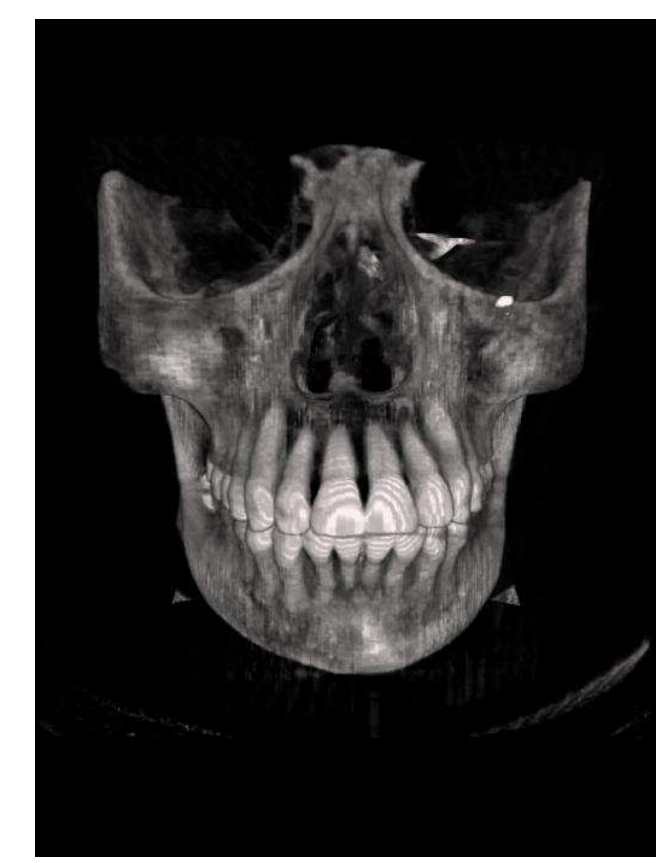

Compressed with ETC1-P 


\section{Rendering Volumetric Datasets}

- 2D texture arrays + compression

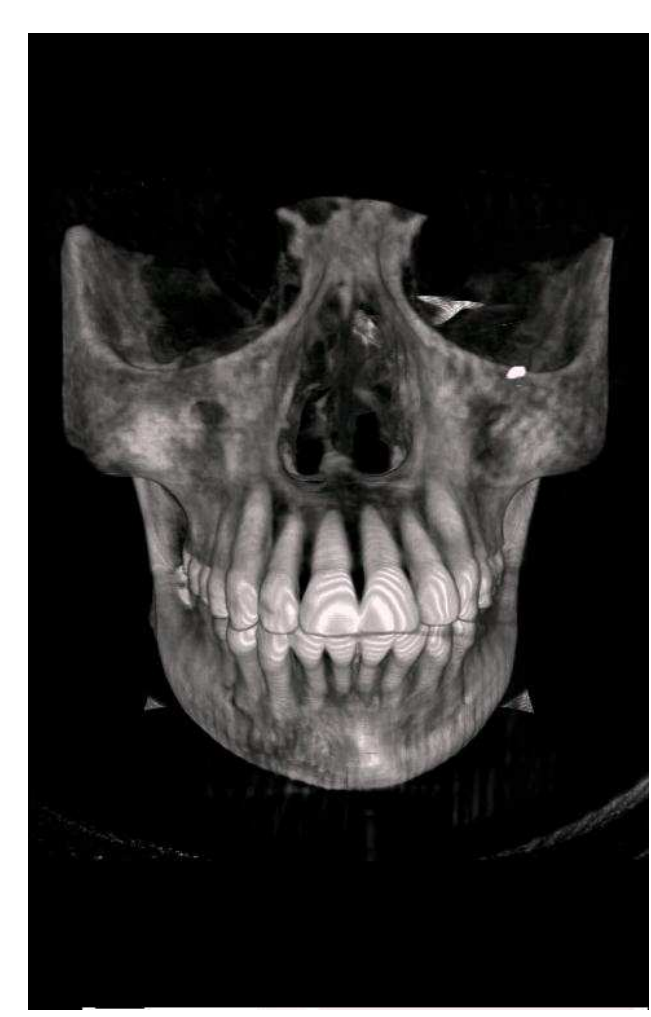

Uncompressed

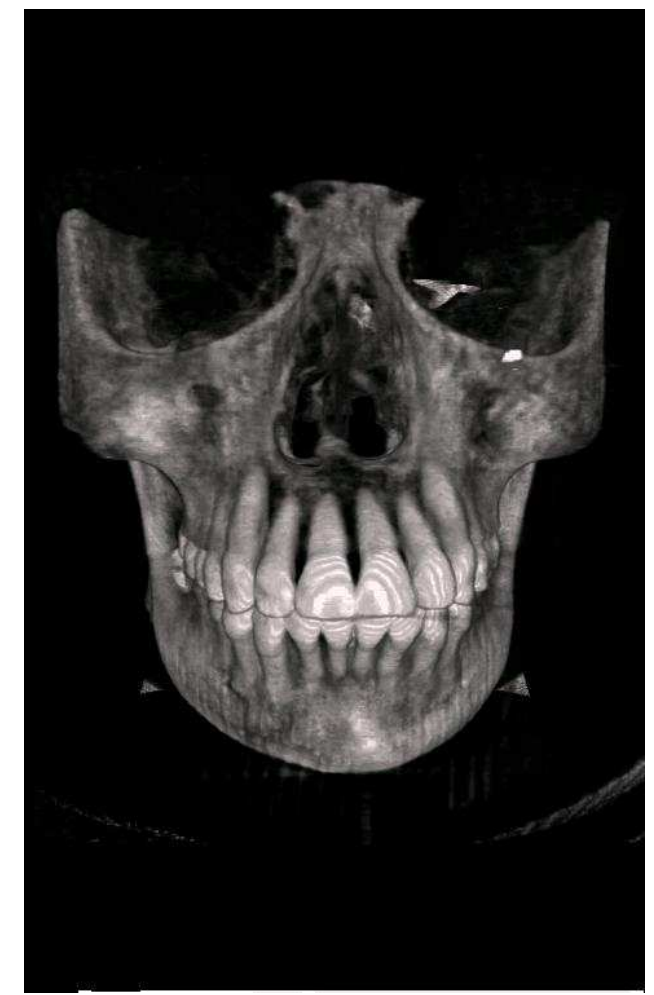

Compressed with PVRTC-4BPP

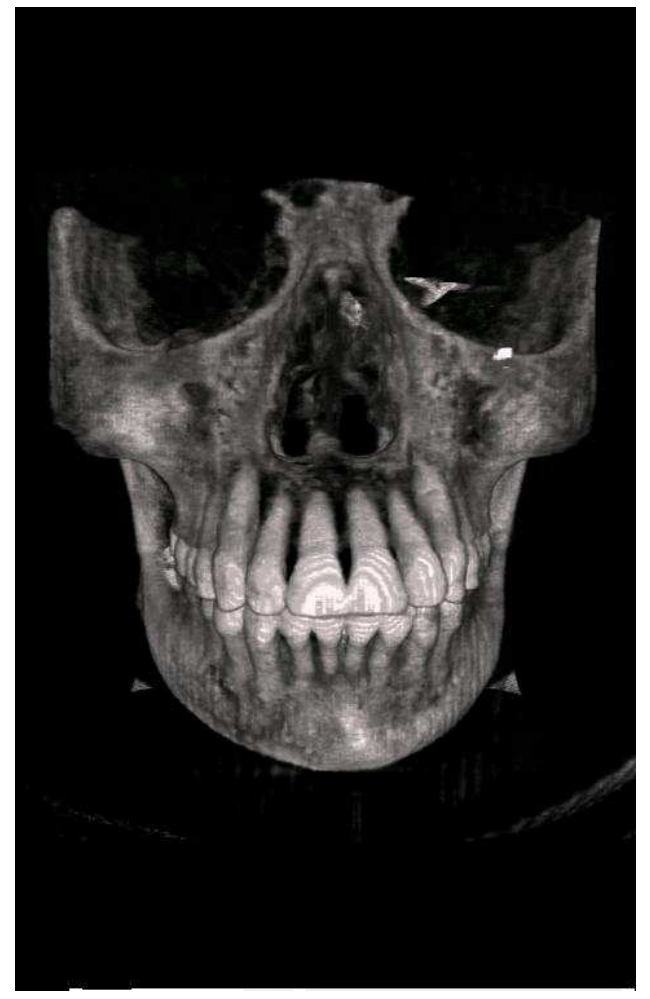

Compressed with PVRTC-2BPP 


\section{Rendering Volumetric Datasets}

- 3D textures [Balsa \& Vázquez, 2012]

- Allow either 3D slices or GPU-based ray casting

- Initially, only a bunch of GPUs sporting 3D textures (Qualcomm's Adreno series >= 200)

- Performance limitations (data: $256^{3}$ - screen resol. 480x800)

- 1.63 for 3D slices

- 0.77 fps for ray casting 


\section{Rendering Volumetric Datasets}

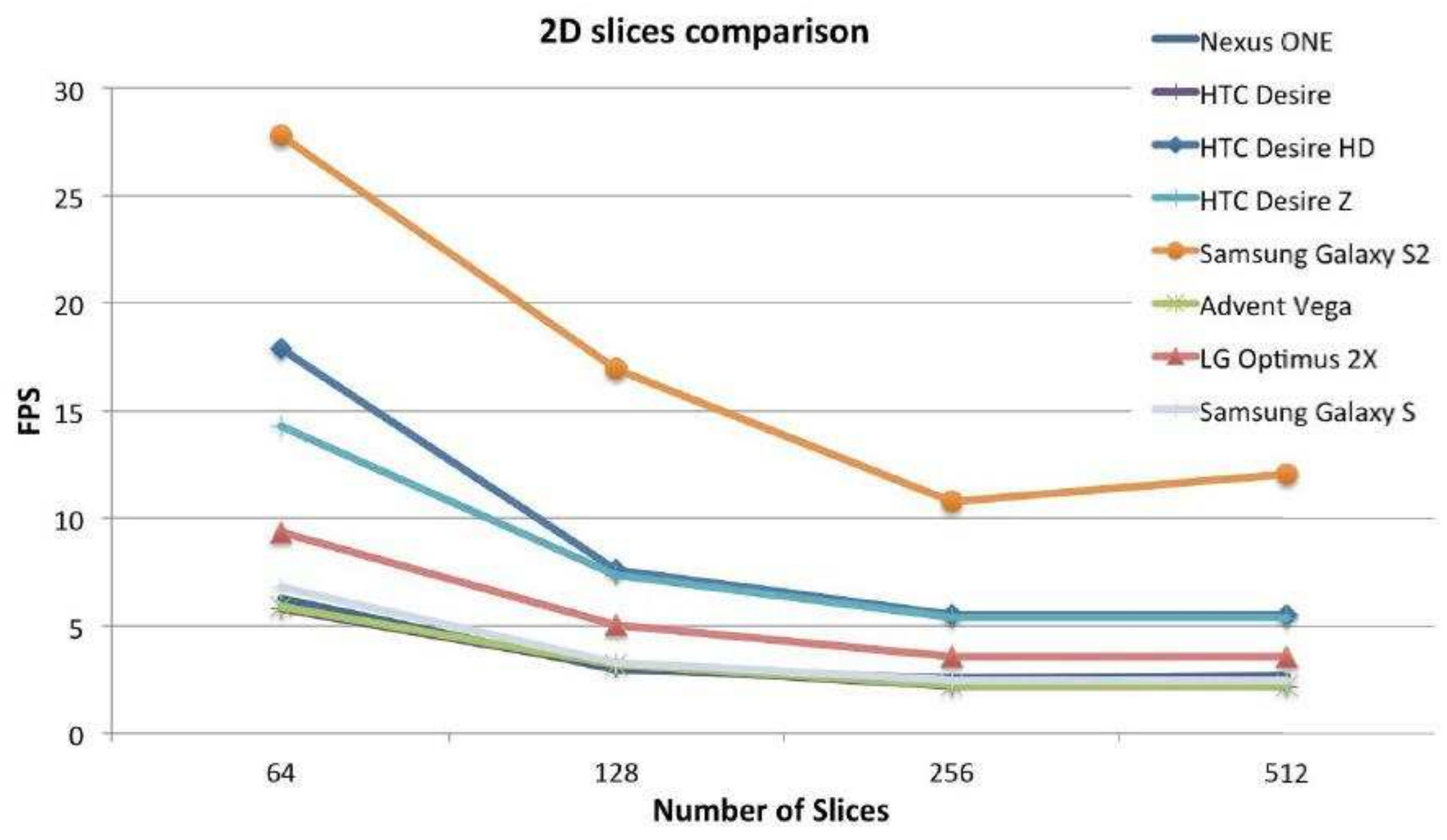




\section{Rendering Volumetric Datasets}

- 2D slices

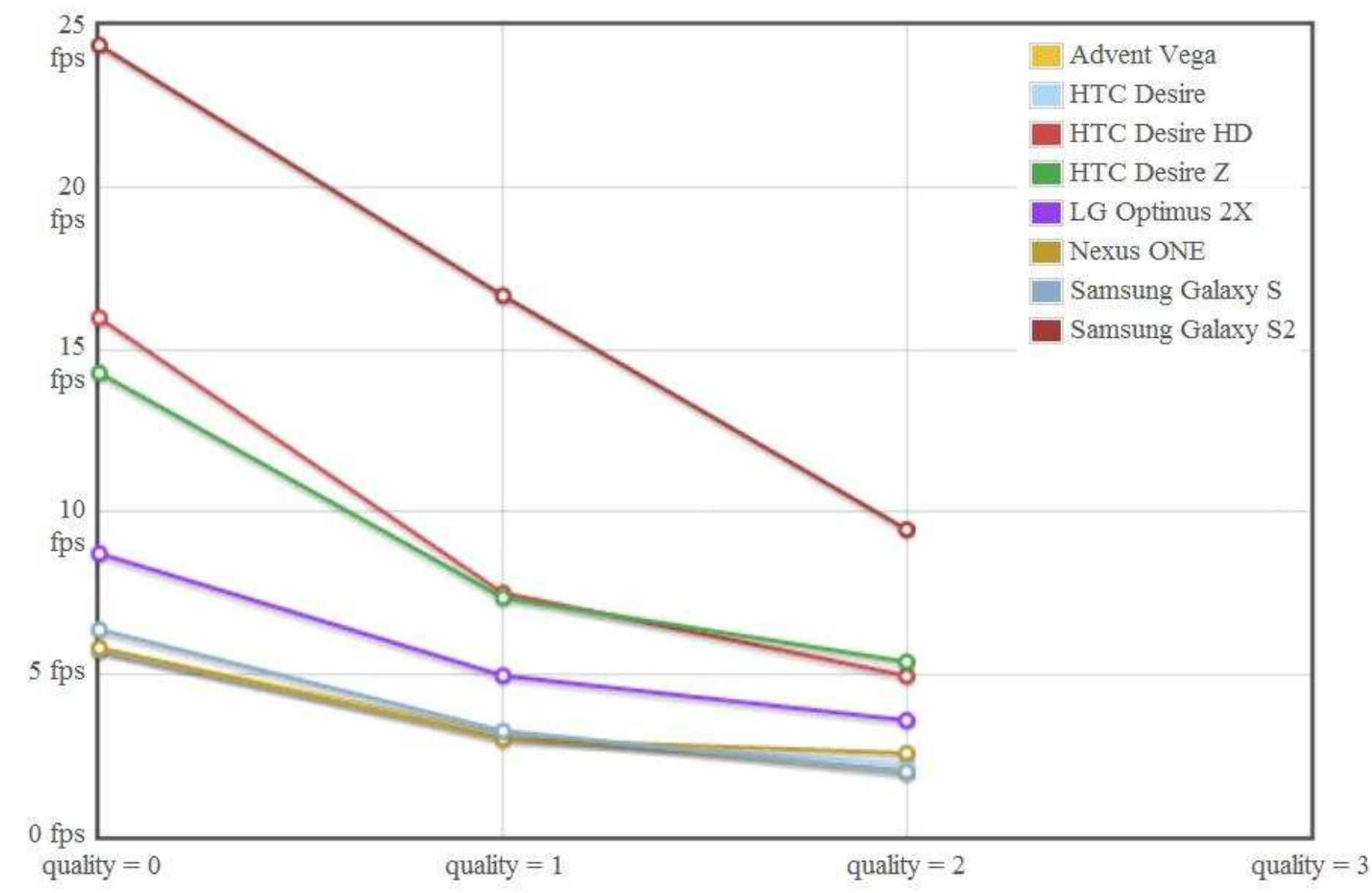




\section{Rendering Volumetric Datasets}

- 2D slices vs 3D slices vs raycasting

Full resolution

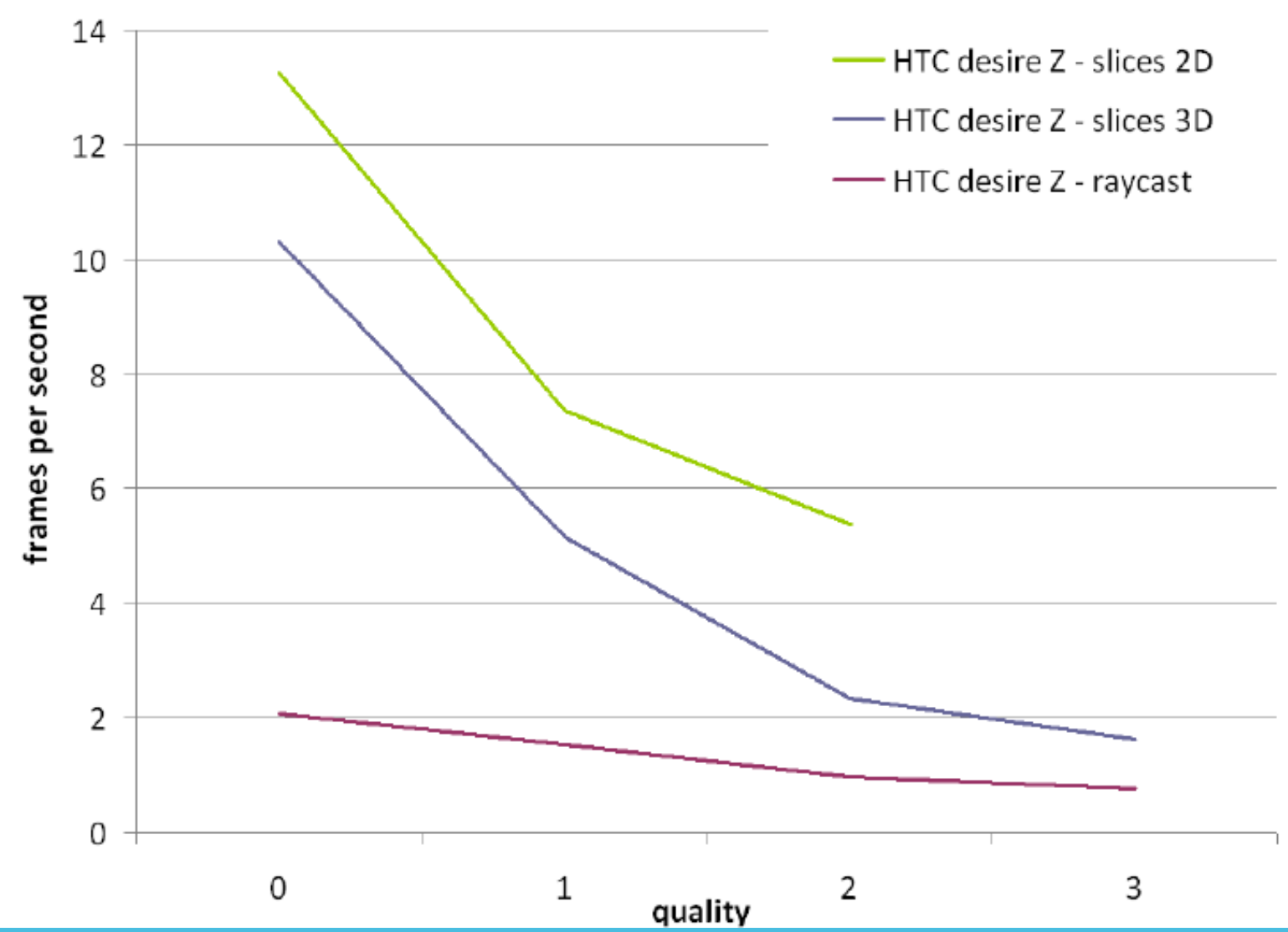




\section{Rendering Volumetric Datasets}

- Using Metal on an iOS device [Schiewe et al., 2015]

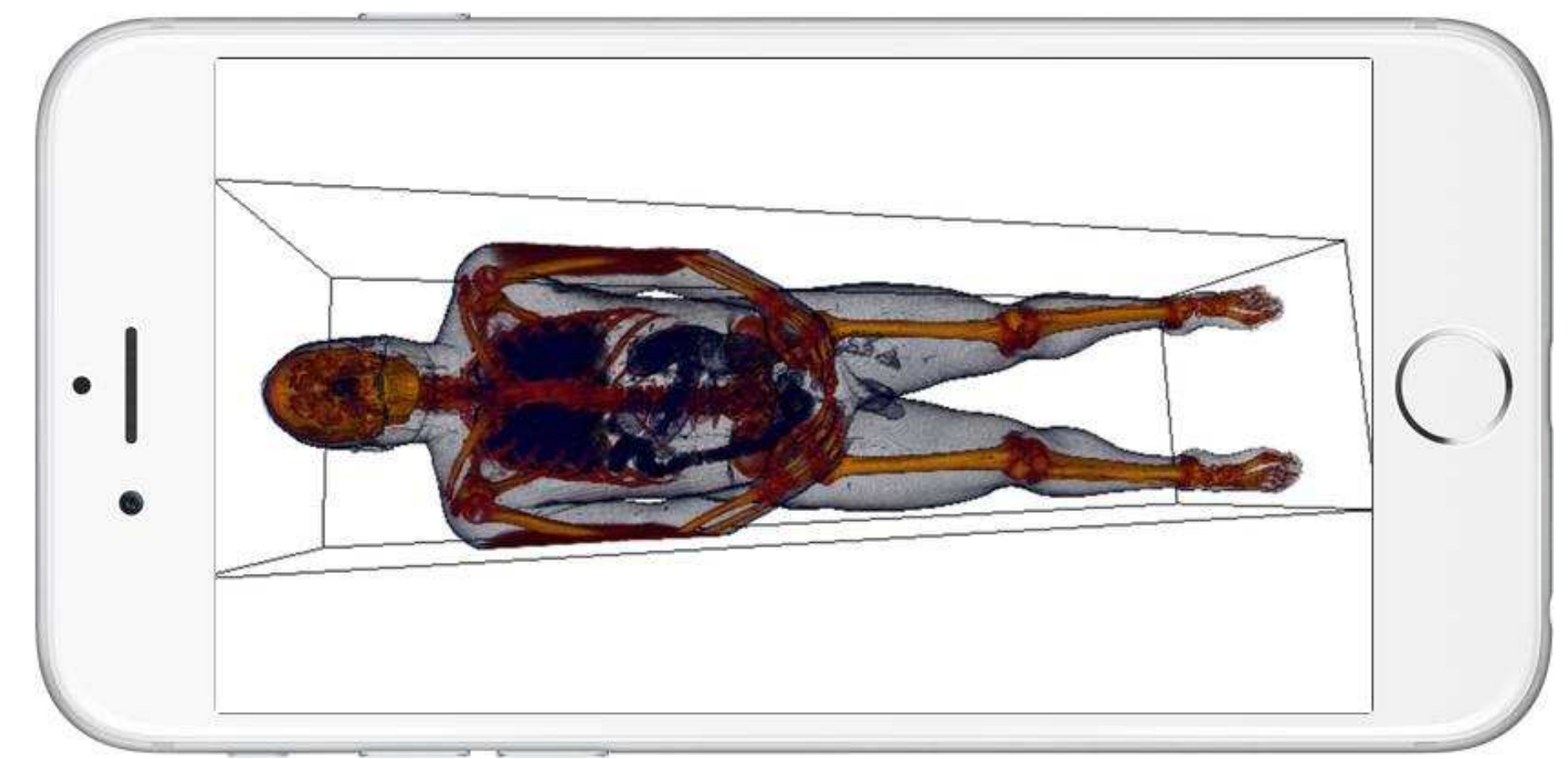

Taken from [Schiewe et al., 2015] 


\section{Volume data. GPU ray casting on mobile}

- Using Metal on an iOS device [Schiewe et al., 2015]

- Standard GPU-based ray casting

- Provides low level control

- Improved framerate (2x, to a maximum of 5-7 fps) over slice-based rendering

- Models noticeably smaller than available memory (max. size was $256^{2}$ x942) 


\section{Rendering Volumetric Datasets}

- Challenges: Transfer Function edition

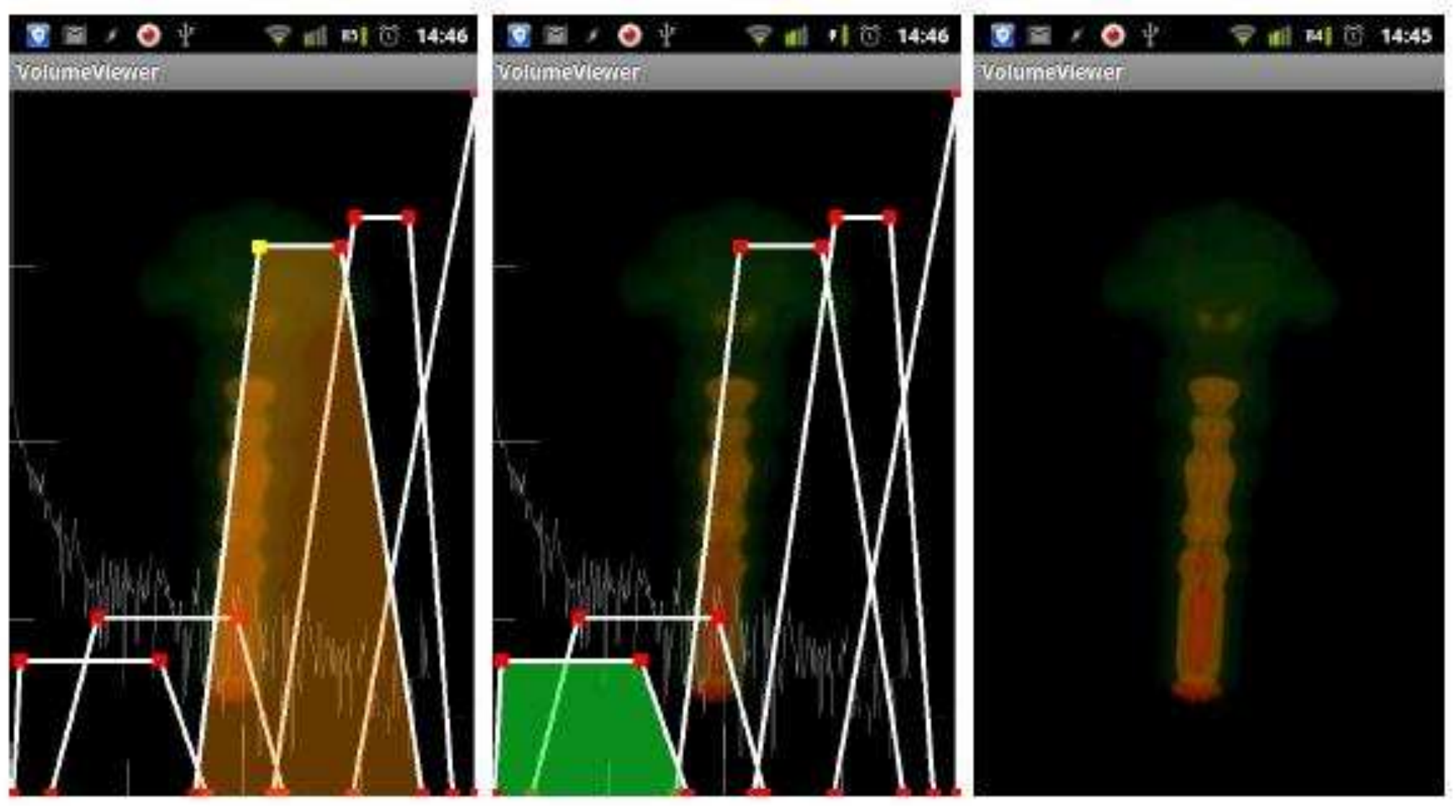




\section{Rendering Volumetric Datasets}

- Challenges: Transfer Function edition

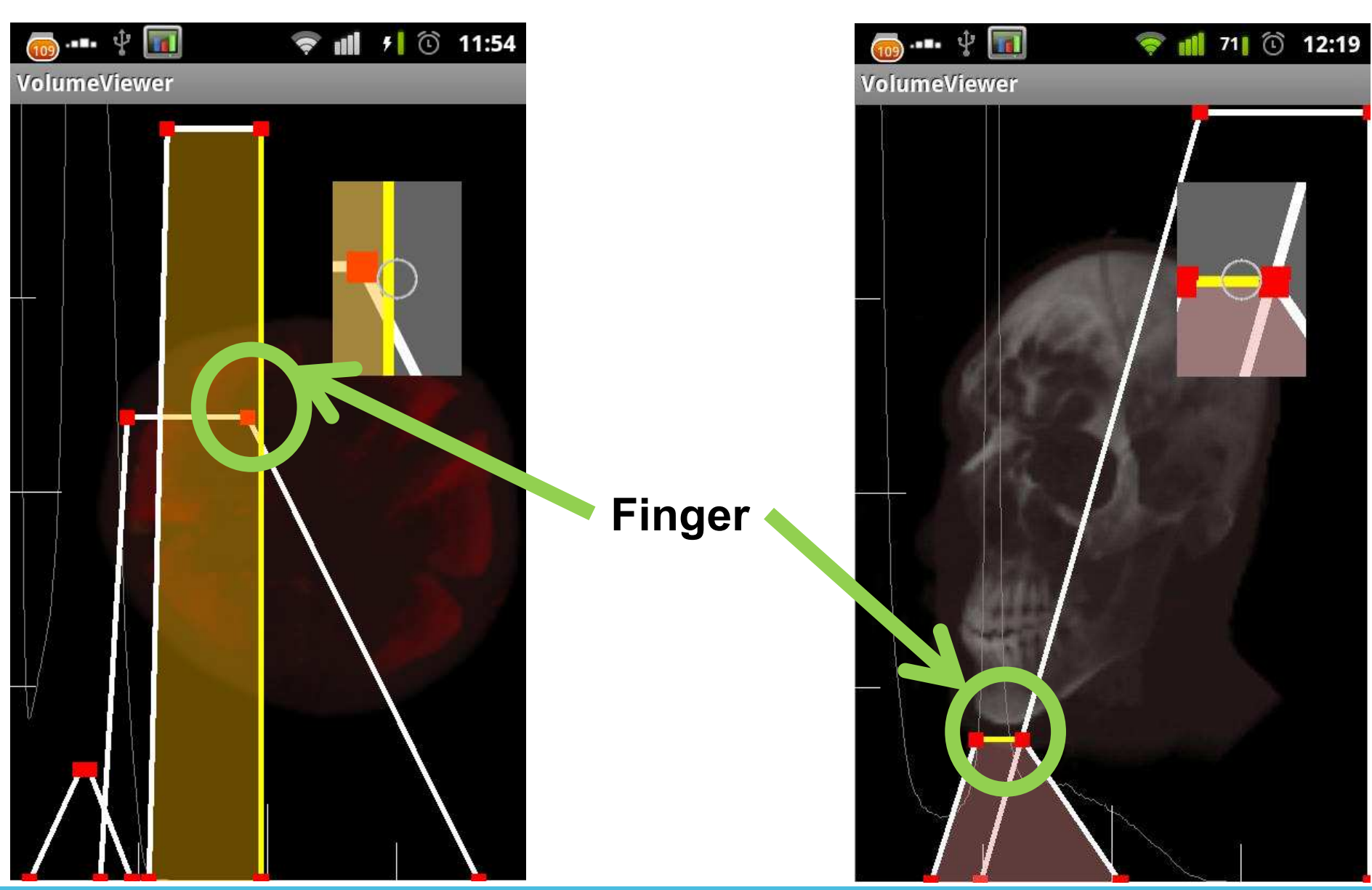




\section{Rendering Volumetric Datasets}

\section{- Conclusion}

- Volume rendering on mobile devices possible but limited

- Can use daptive rendering (half resolution when interacting)

- 3D textures in core GLES 3.0

- Still limited performance ( 7fps...)

- Interaction still difficult

- Client-server architecture still alive

- Can overcome data privacy/safety \& storage issues

- Better 4G-5G connections

. 
Next Session

MOBILE METRIC CAPTURE AND RECONSTRUCTION 


\section{Part 5.1}

Mobile Metric Capture \& Reconstruction: Introduction

Enrico Gobbetti, CRS4 


\section{Computer vision and mobile applications}

Image Search

Material

Capture

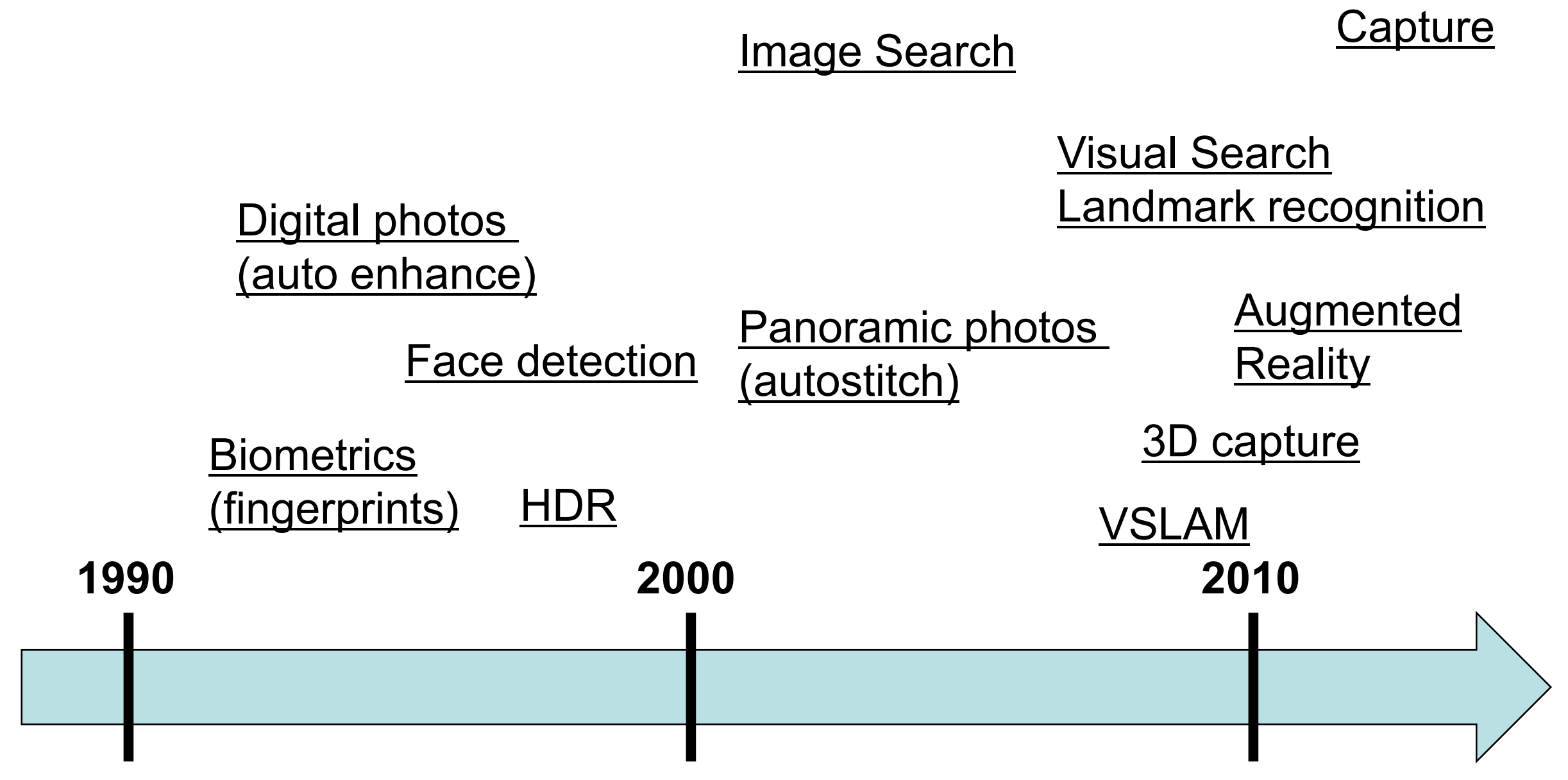




\section{Computer vision and mobile applications}

- Mostly 2D

- Image enhancement

- Image stitching

- Image matching

- Object detection

- Texture classification

- Activity recognition

$-\ldots$
- Mostly 3D

- Camera localization

- Pose estimation

- 3D shape recovery

- 3D scene reconstruction

- Material/appearance recovery

- Augmented reality

$-\ldots$ 
Applications made possible by specific features of mobile devices!

- Features

1. Mobility

2. Camera

3. Active light

4. Non-visual sensors

5. Processing power

6. Connectivity

7. Display
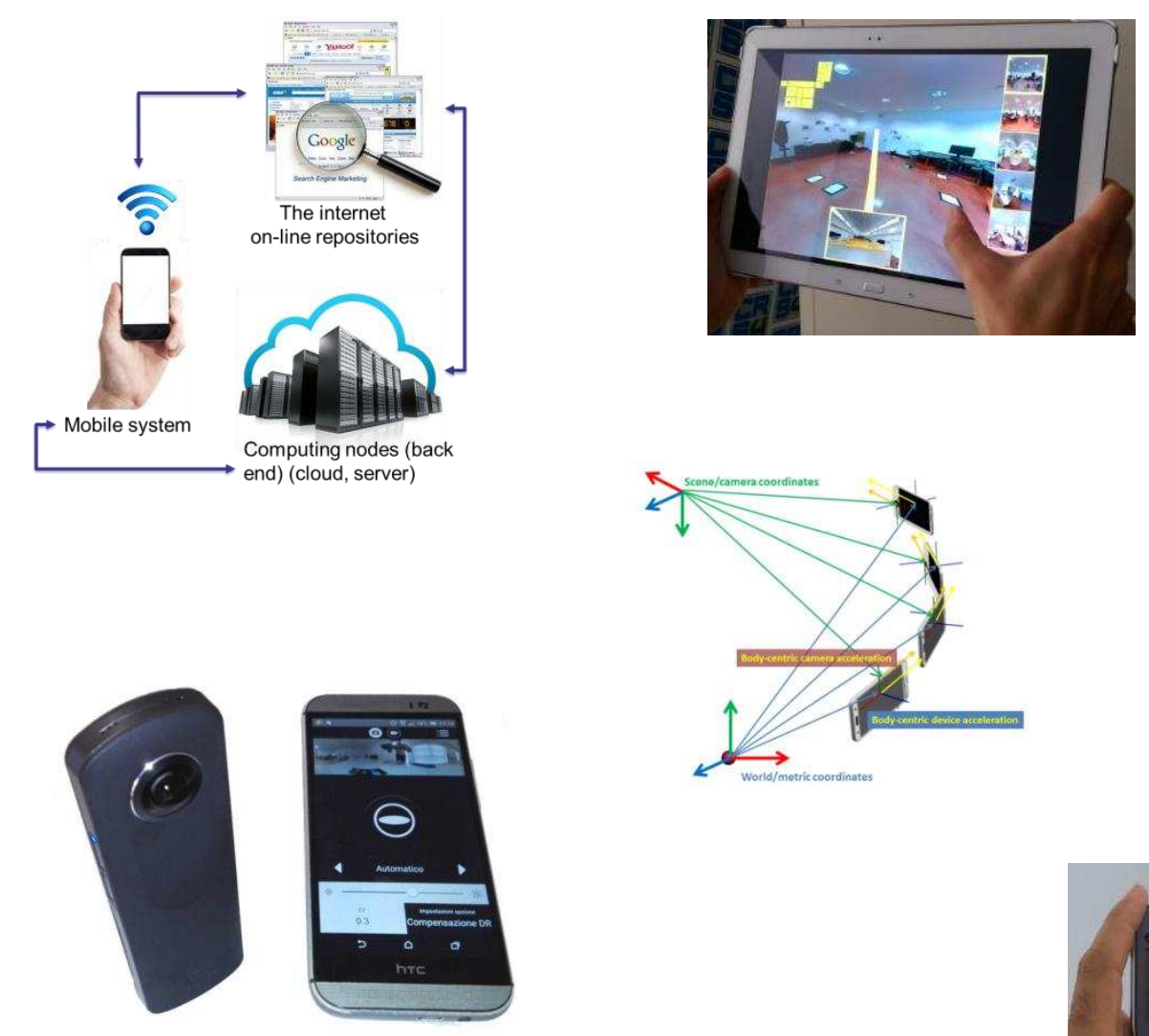
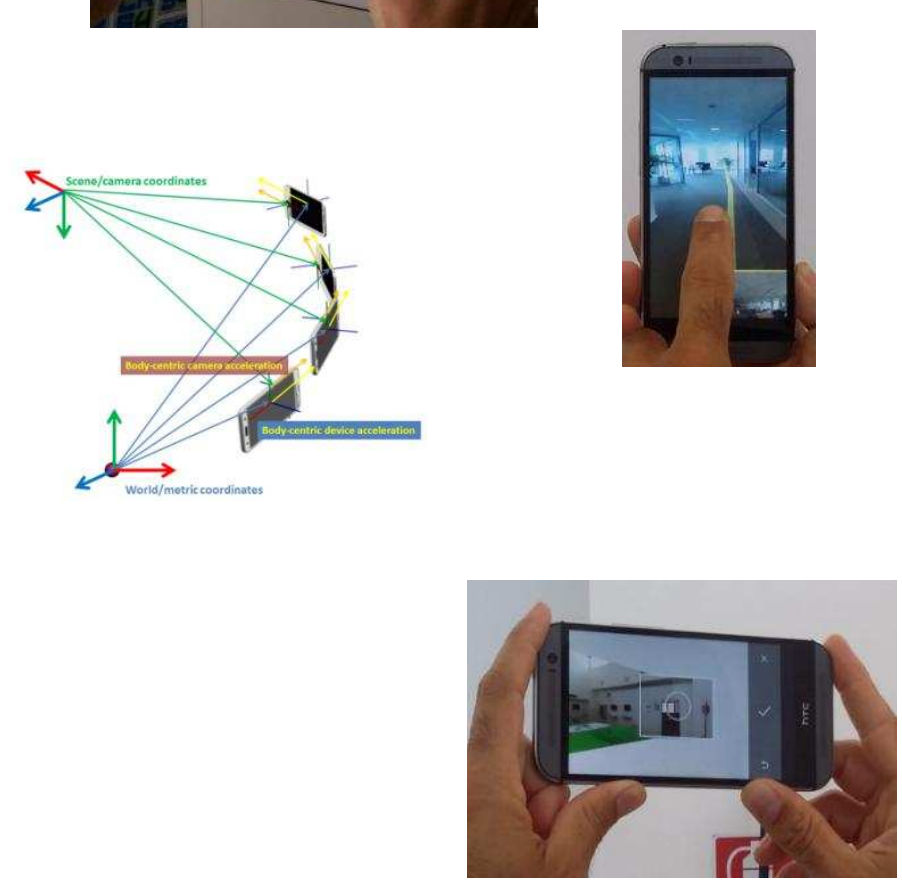


\section{Features (1/7): Mobility}

- Consumer

- Smartphones

- Tablets

- Embedded

- Autonomous driving

- Assistive technologies

- Specific

- Drones

- Robots

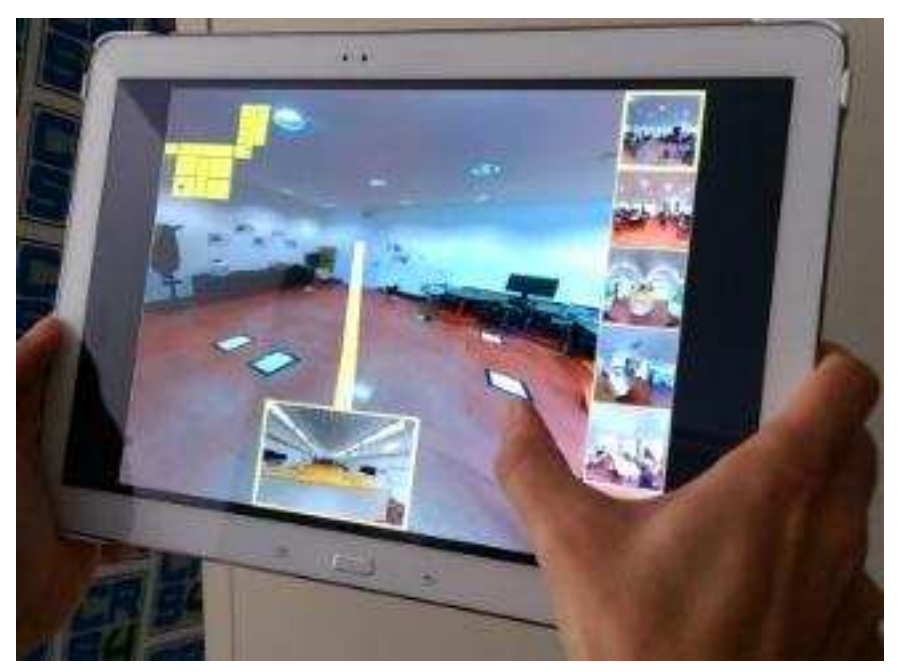




\section{Features (1/7): Mobility}

- Consumer

- Smartphones

- Tablets

- Embedded

On-site applications / Personal

- Autonomous driv applications / Motion and/or location

- Assistive technol taken into account / Embedded solutions

- Specific

- Drones

- Robots

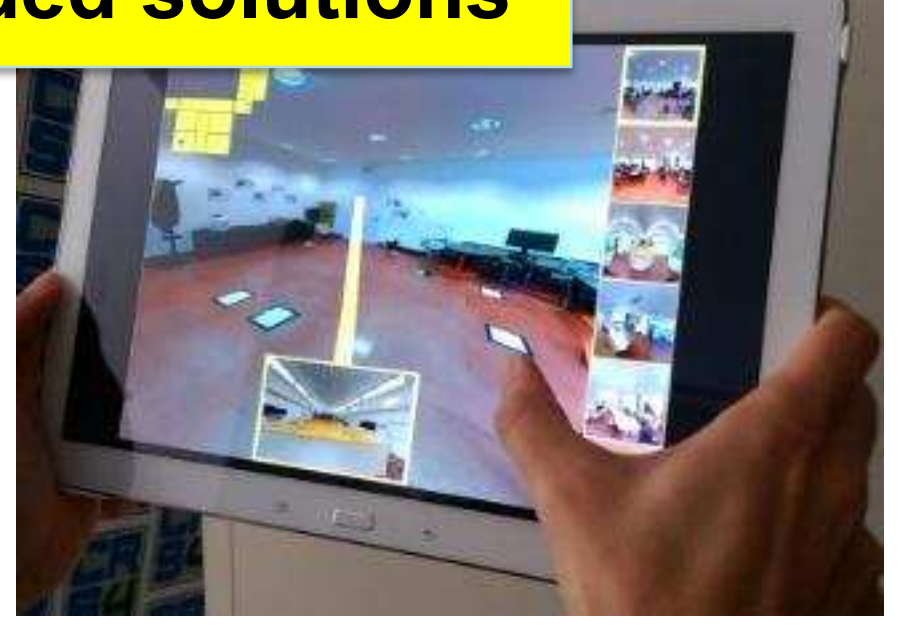




\section{Features (2/7): High-res/flexible camera}

\section{- Common features}

- High resolution and good color range (>12 MP, HDR)

- Small sensors (similar to point and shoot cameras approx. 1/3")

- High video resolution and frame rate (4K at 30fps)

- Wide variety of field of views

- standard, fisheye, spherical

- Specialized embedded cameras...

- Better lenses and sensors...

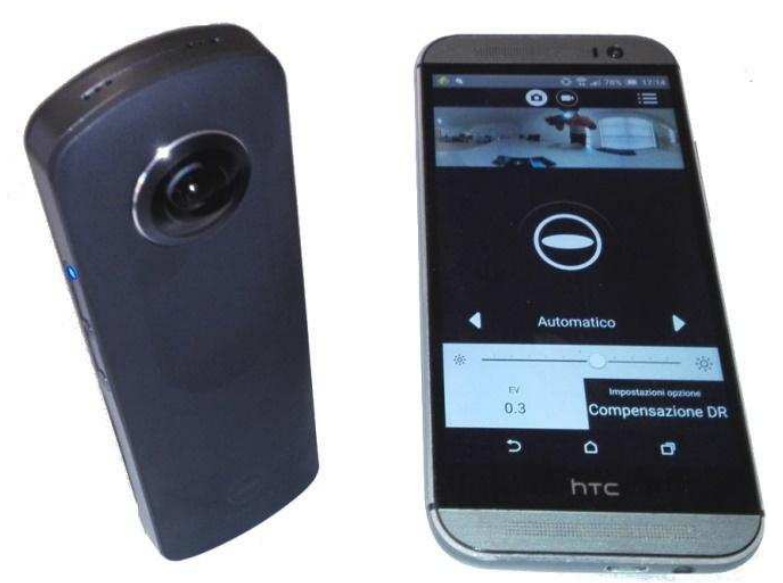




\section{Features (2/7): High-res/flexible camera}

- Common features

- High resolution and good color range (>12 MP, HDR)

- Small sensors (similar to point and shoot cameras approx. 1/3")

- High video resolution and frame rate (4K at 30fns)

- Wide variety of Visual channel is the primary one Computational photography

- standard, fishey

- Specialized eml Apps analyze/use snapshots or videos

- Better lenses and sensors... 


\section{Features (3/7): Active lighting}

- All smartphones have a flashlight

- LED source at fixed distance from camera

- Custom devices have integrated emitters

- Google TANGO / Microsoft Kinect

- Integrated depth sensor

- Leads to specialized capture procedures

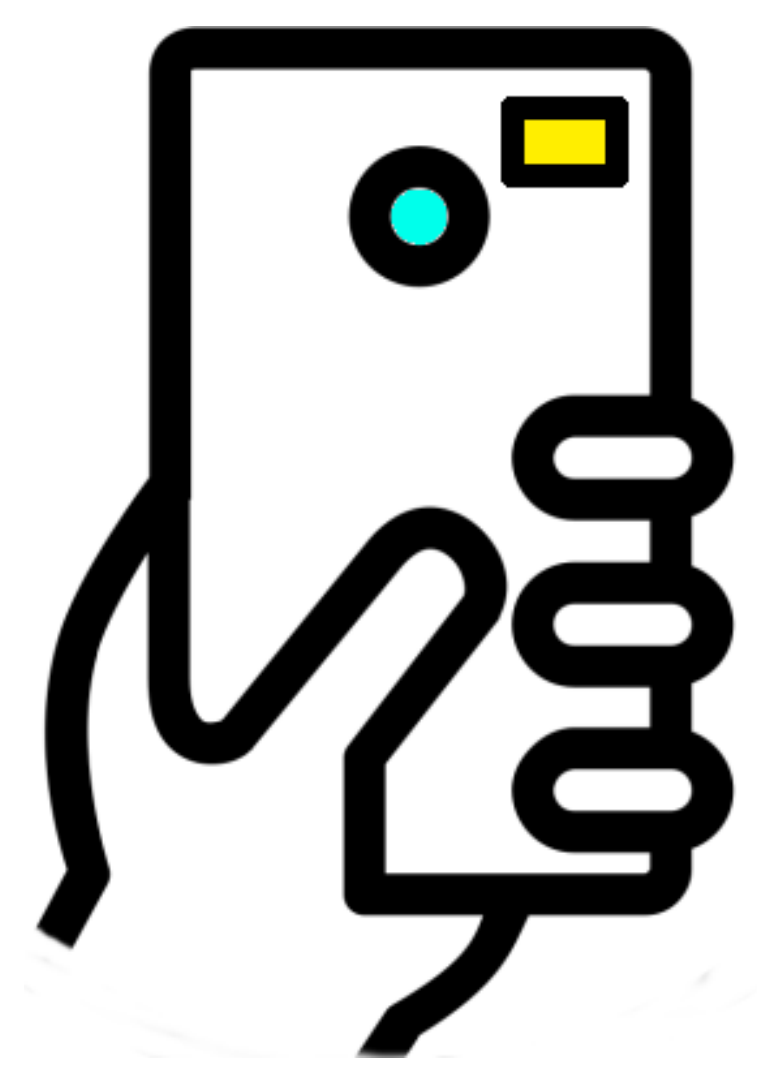




\section{Features (3/7): Active lighting}

- All smartphones howo s flochlinht

- LED source at fix Specialized capture procedures

- Custom devices exploiting synchronization of illumination emitters

and visual sensing

- Google TANGO / Ex. Riviere et al. Mobile surface reflectometry. Computer

- Integrated dept Graphics Forum. 2015.

- Leads to specia procedures

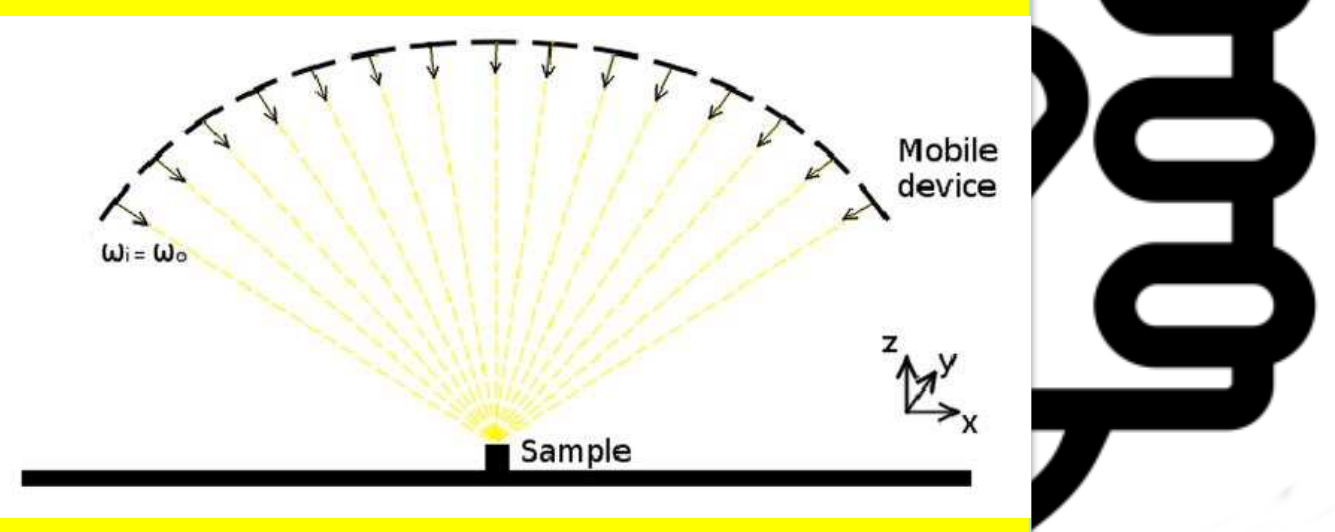




\section{Features (4/7): Non-visual sensors}

- Absolute reference

- GPS / A-GPS

- Mainly for outdoor applications

- Magnetometer

- Enable compass implementation

- Often inaccurate for indoor

- Relative reference

- Accelerometer

- Variable accuracy (sensitive to temperature)

- Good metric information for small scale scene

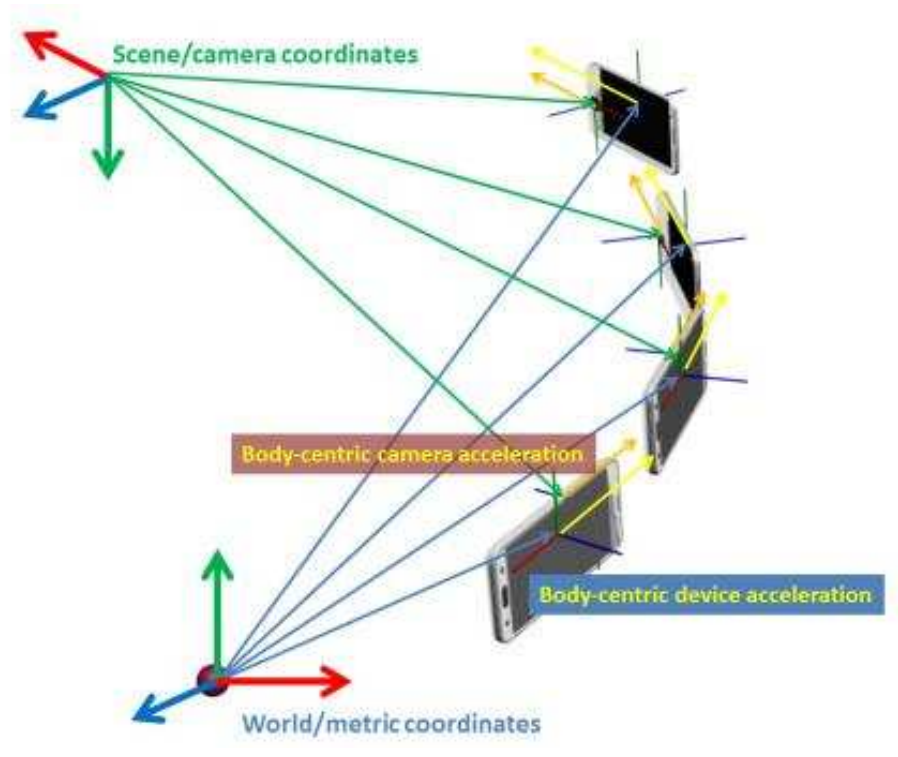

- Gyroscope

- Very good accuracy for device relative orientation

- Synced with camera! 


\section{Features (4/7): Non-visual sensors}

- Absolute refereı Data fusion!

- GPS / A-GPS

- Mainly for outd Ex. Garro et al. Fast Metric Acquisition with Mobile

- Magnetometer

Devices. VMV 2016

- Enable compas

- Often inaccura

- Relative referen

- Accelerometer

- Variable accur:

- Good metric in o

- Gyroscope
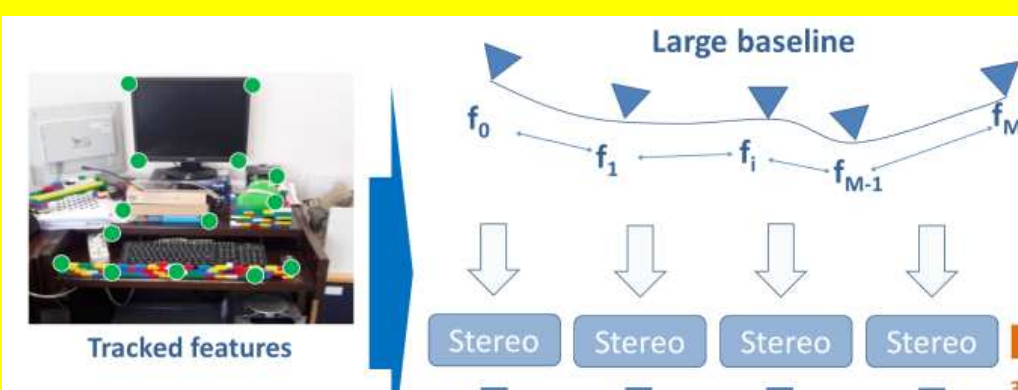

- Very good accuracy ror uevice rerative orieriation
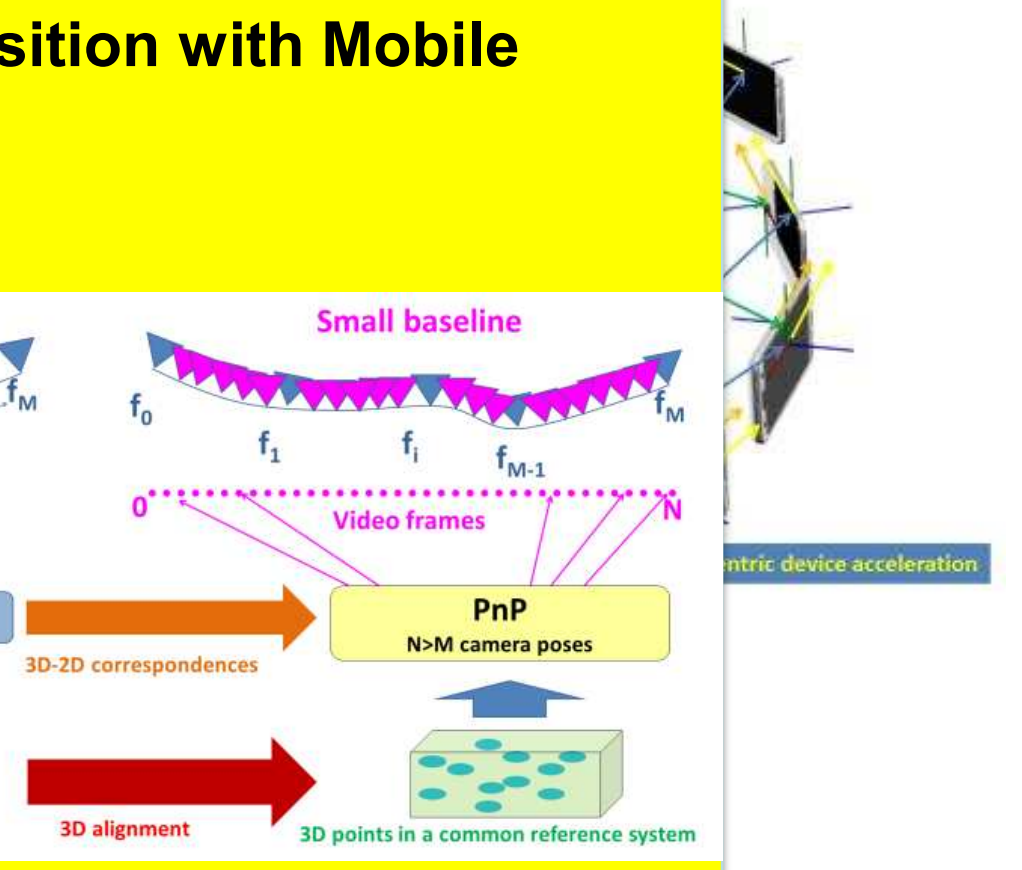

- Synced with camera! 


\section{Features (5/7): Processing power}

- Growing performance of mobile CPU+GPU

- (see previous sections)

- Capable to execute computer vision pipeline on mobile device - i.e. OpenCV for Android

- Some limitations due to power consumption

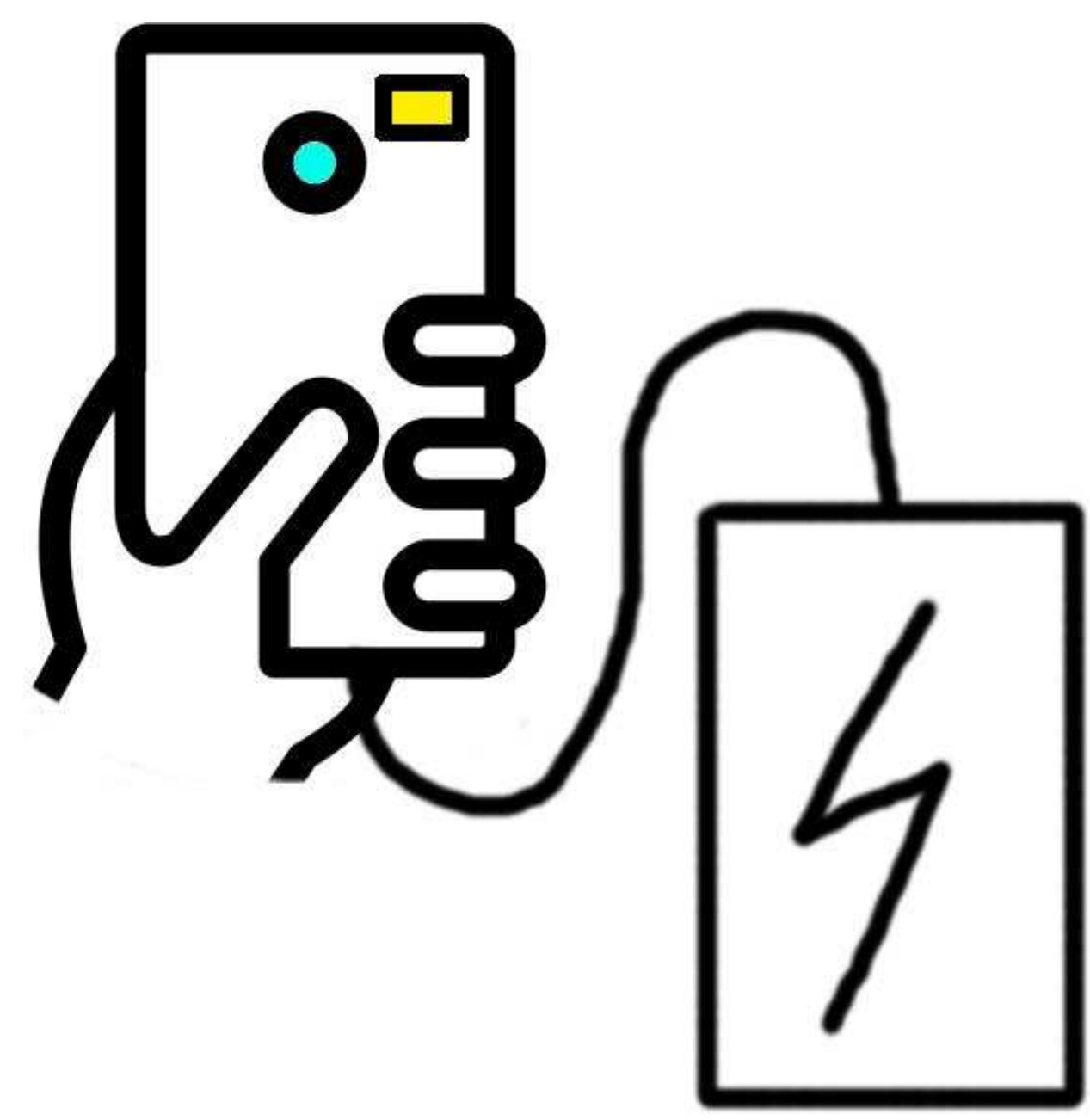




\section{Features (5/7): Processing power}

- Growing perforr On-board pre-processing or even full CPU+GPU processing

- (see previous ser

- Capable to exec Ex. Tanskanen et al. Live Metric 3D Reconstruction on vision pipeline $c$ - i.e. OpenCV for $t$.

- Some limitation: consumption Mobile Phones. ICCV2013
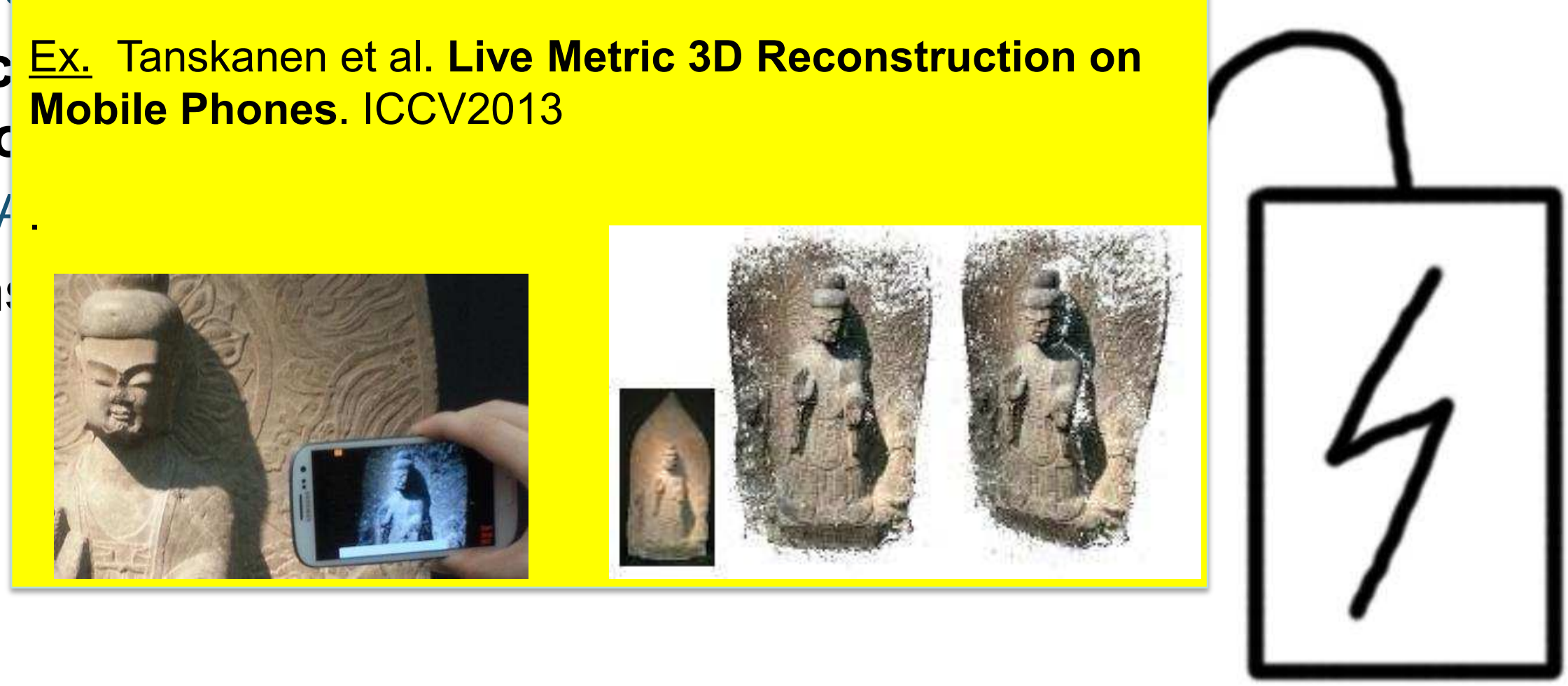


\section{Features (6/7): Connectivity}

- Many connectivity options

- Local area: NFC, Bluetooth, Bluetooth Low Energy, Wi-Fi 802.11x

- Wide area: Cellular wireless networks: $3 \mathrm{G} / 4 \mathrm{G} / 5 \mathrm{G}$

- Mobile devices can connect at local or wide area at reasonable speed

- Typical LTE/4G: 18 Mbps down, 9.0 Mbps up

- Typical Wi-Fi: 54Mbps (g), 300Mbps (n), $1 \mathrm{Gbps}$ (ac).

- Lo-cost $\rightarrow$ No-Costs

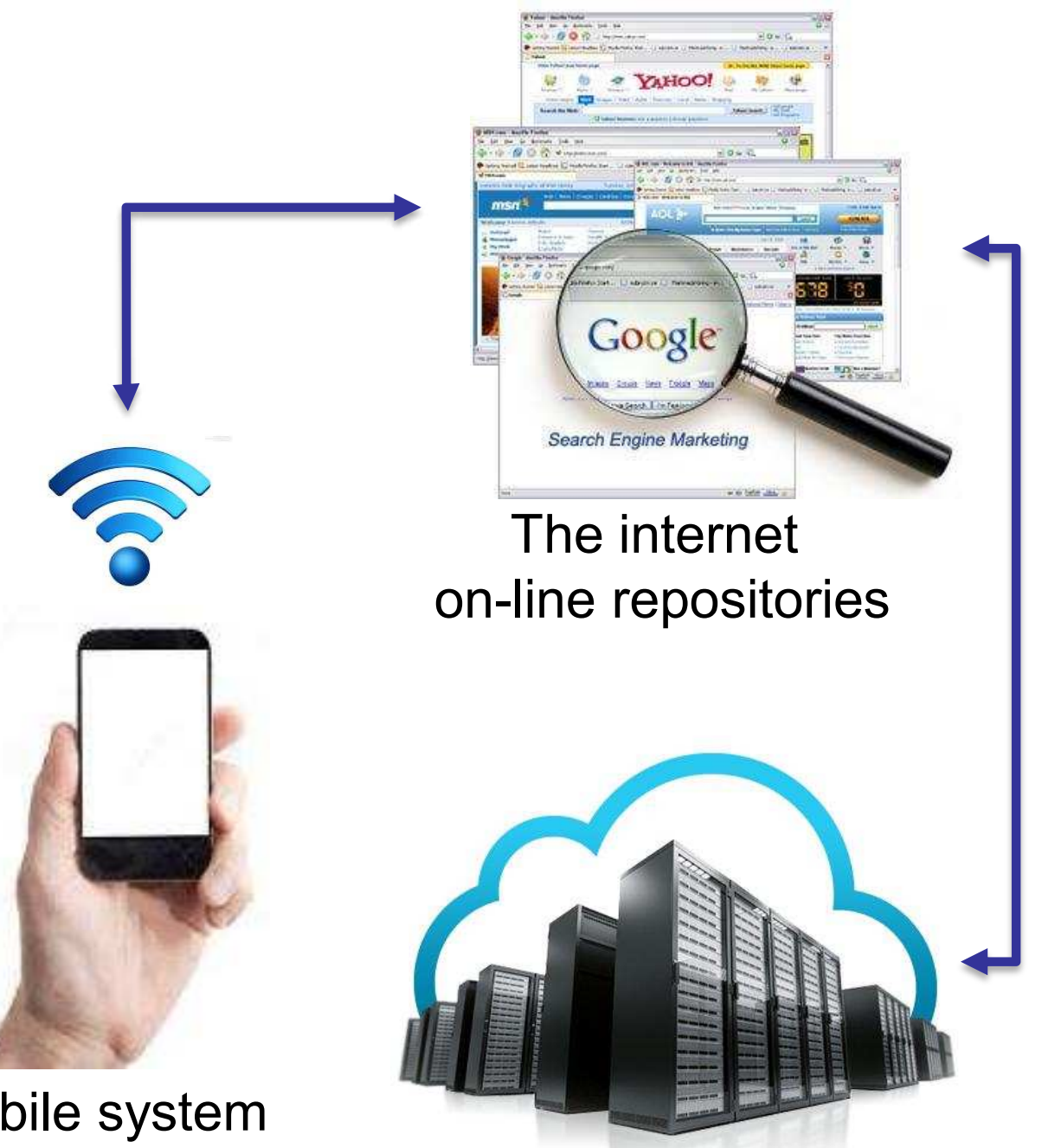

Computing nodes (back end) (cloud, server) 


\section{Features (6/7): Connectivity}

- Many connectiv Load balancing (client / server)

- Local area: NFC, Access to large databases (e.g., search) Low Energy, Wi-l Communication

- Wide area: Cellul $3 \mathrm{G} / 4 \mathrm{G} / 5 \mathrm{G}$

Ex. Gammeter et al. Server-side object recognition and

- Mobile devices 1 local or wide are speed

- Typical LTE/4G: up

- Typical Wi-Fi: 54 $1 \mathrm{Gbps}(\mathrm{ac})$.

client-side object tracking for mobile augmented reality. CVPRW 2010.

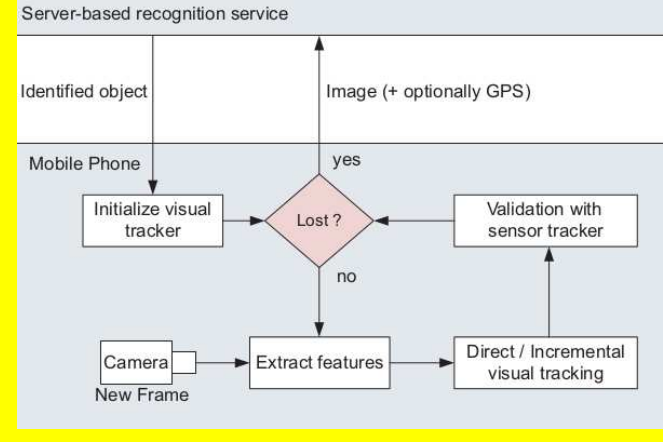

Lo-cost -> No-Costs

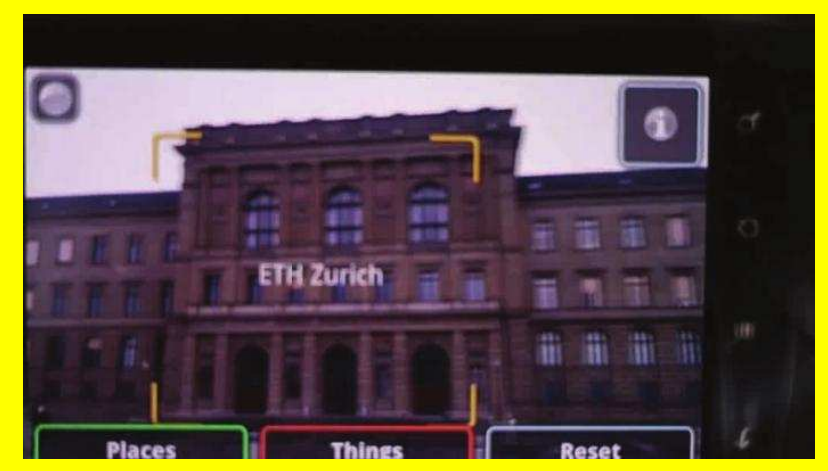

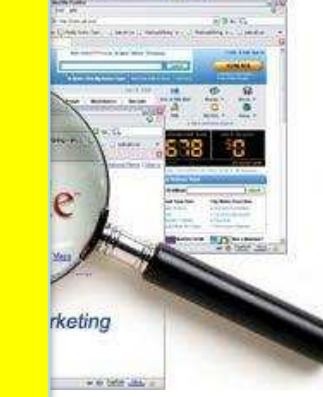

rnet

ositories

Computing nodes (back end) (cloud, server) 


\section{Features (7/7): Display!}

- Hi-res/hi-density display

- Data presentation!

- Large with respect to processing power

- Co-located with camera + other sensors

- Tracking during capture!

- Touch screen

- Co-located user-interface

- Small with respect to fingers (precision, occlusions!)

- (UI also may exploits other sensors)
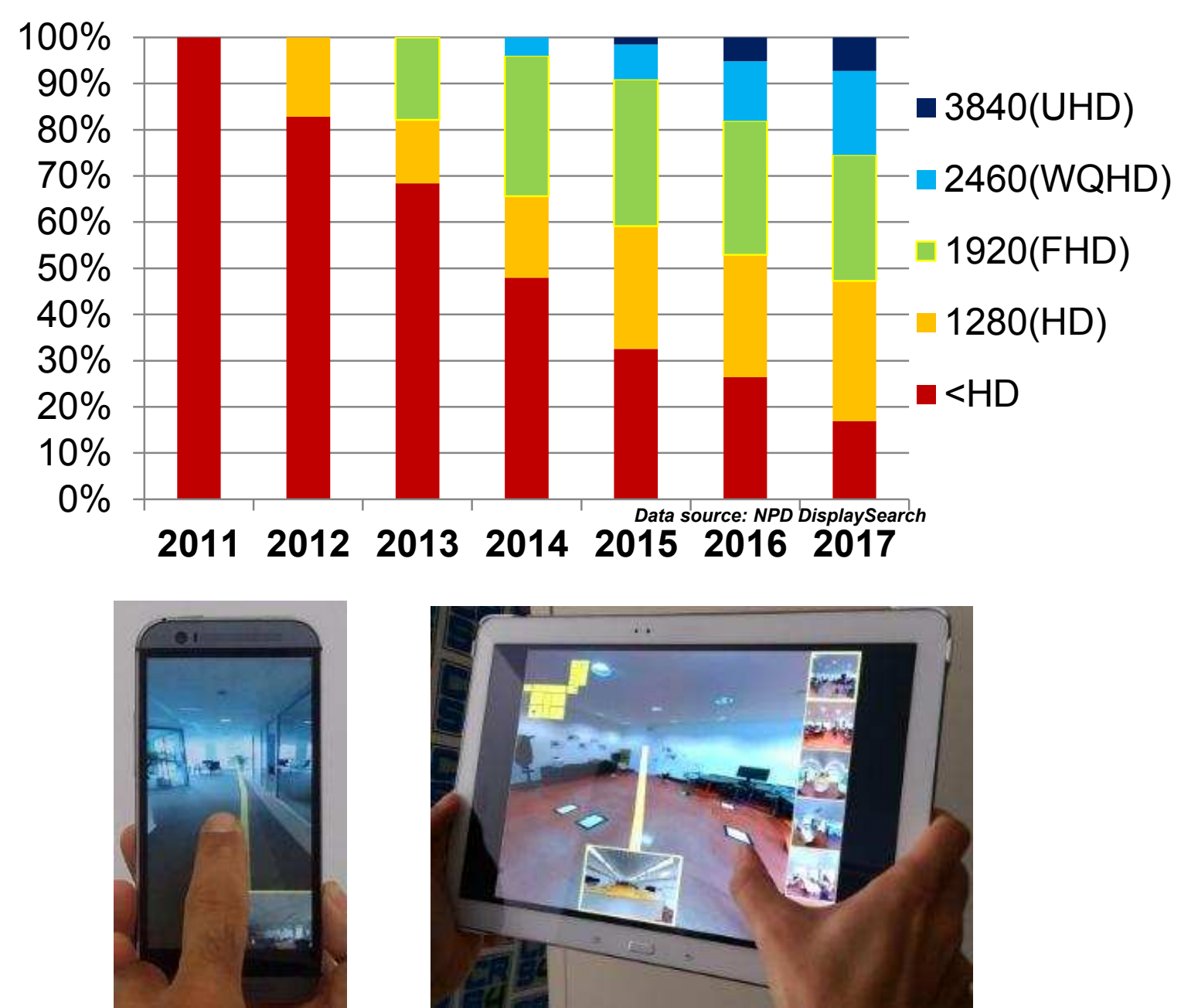


\section{Features (7/7): Display!}

- Hi-res/hi-densit] Data/result presentation

- Data presentatiol Guided capture / Augmentation

- Large with respe

- Co-located with Ex. Pintore et al. Mobile Mapping and Visualization of sensors

- Tracking during $c$

- Touch screen

- Co-located user-

- Small with resper occlusions!) Indoor Structures to Simplify Scene Understanding and Location Awareness. ECCV ACVR 2016
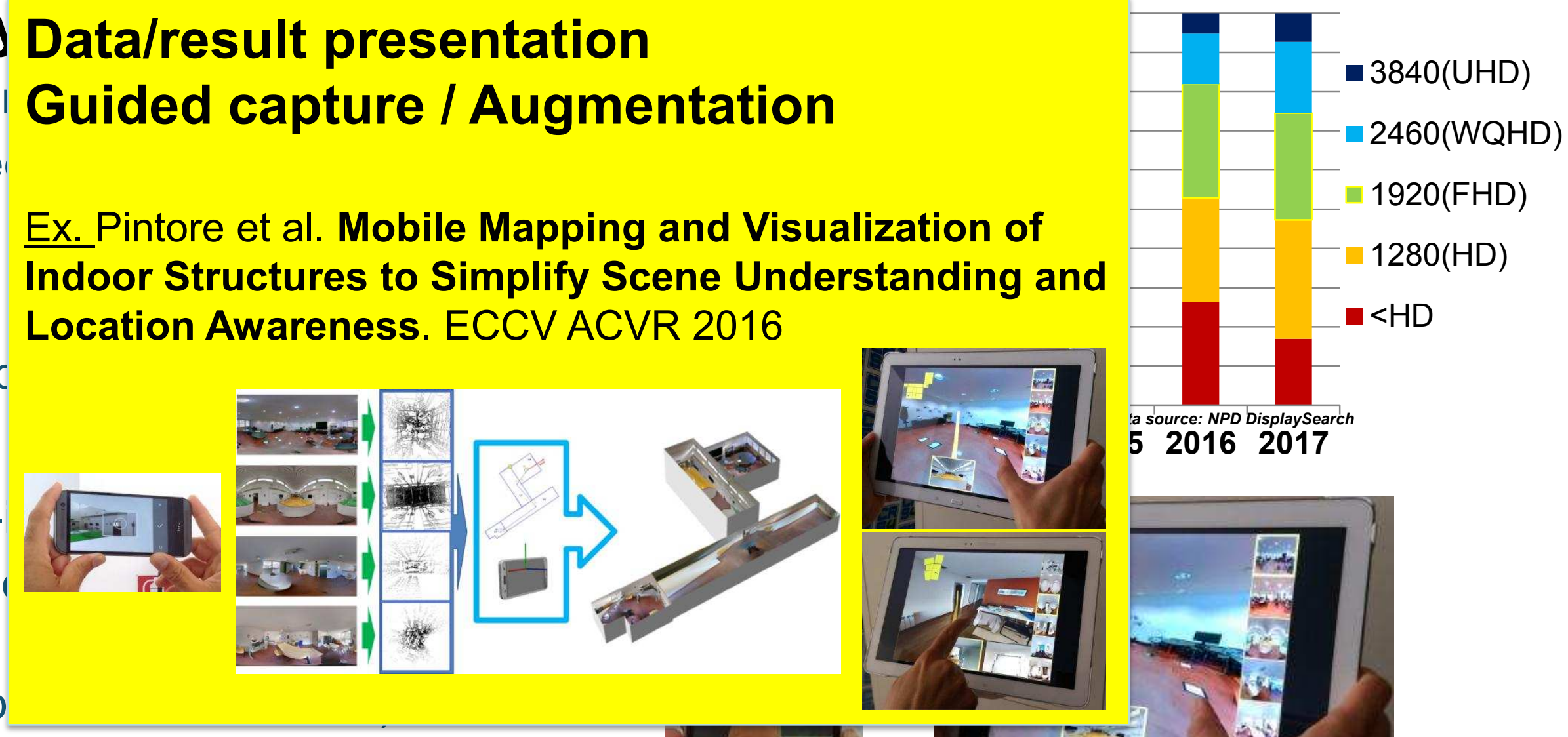

1920(FHD)

1280(HD) $-<\mathrm{HD}$

- (UI also may exp
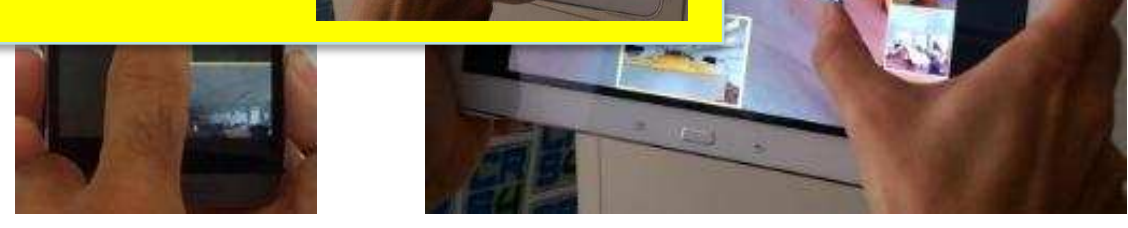
Wrap-up: mobile apps characterized by the exploitation of mobile device features

- Features

1. Mobility

2. Camera

3. Active light

4. Non-visual sensors

5. Processing power

6. Connectivity

7. Display

Next session: case studies!
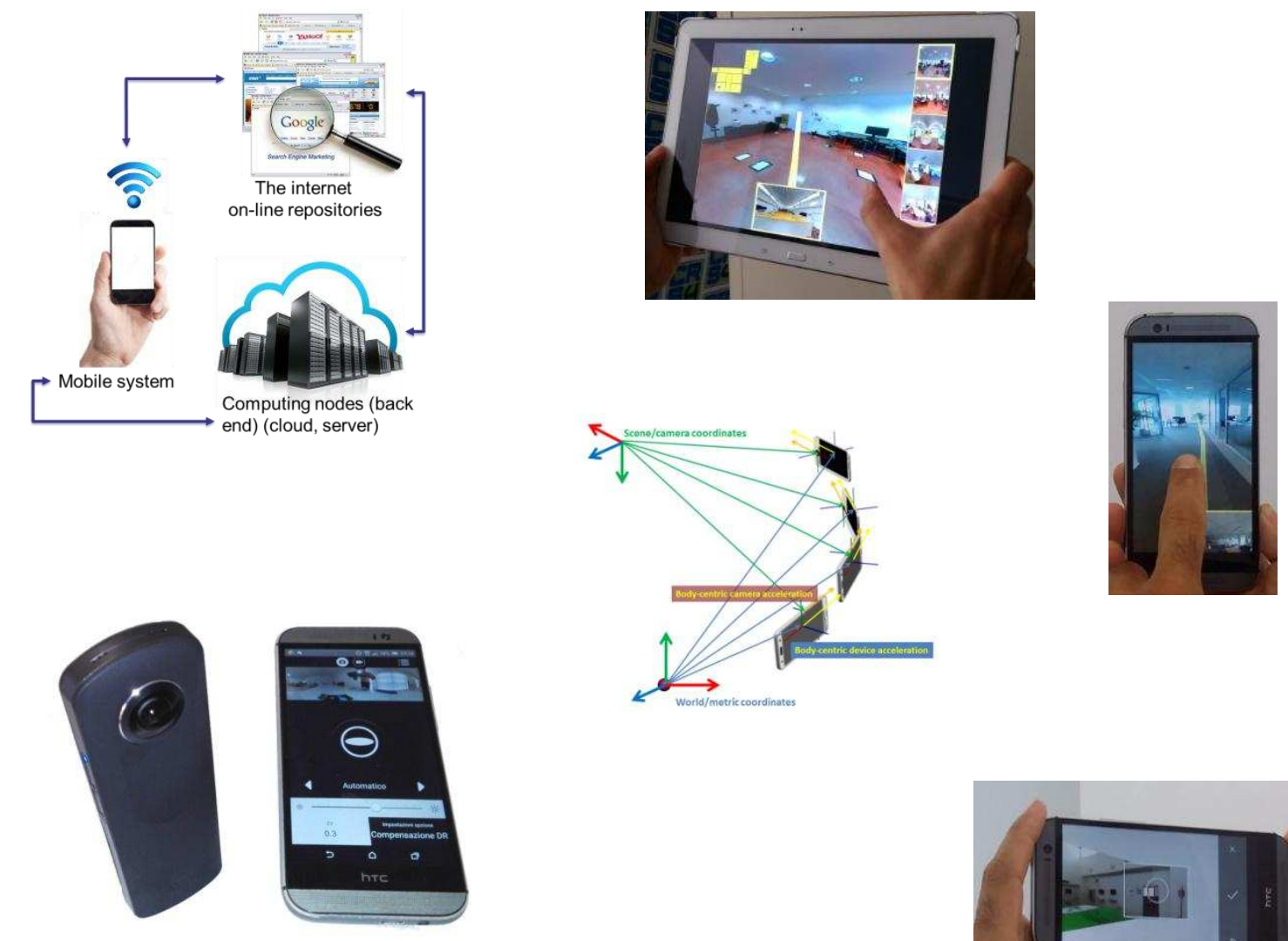

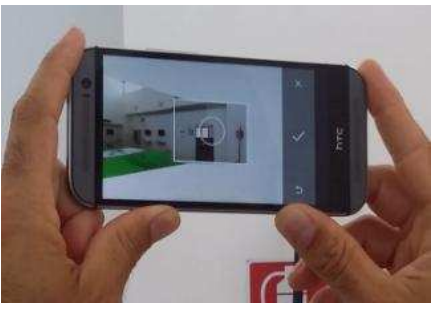




\section{Part 5.2}

Mobile Metric Capture \& Reconstruction: Case studies in metric capture

\section{Giovanni Pintore, CRS4}




\section{Example 1}

\section{DATA FUSION FOR METRIC CAPTURE}




\section{Metric acquisition with a commodity mobile phone}

- Goal

- Capture 3D models with real-world measures

- Data fusion approach

- Exploit synchronization of visual sensor \& IMU to capture scenes in real-world units
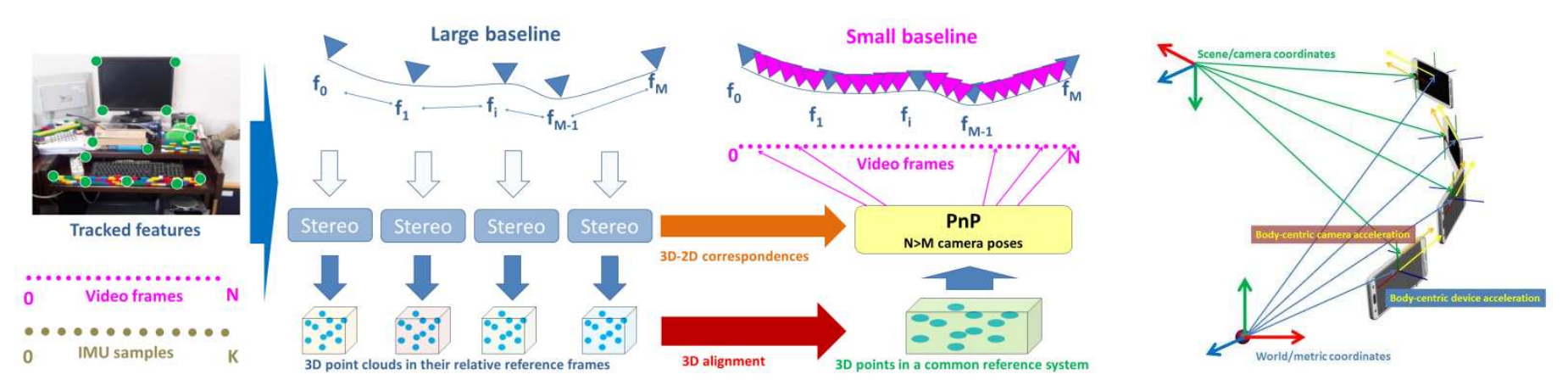

Garro et al. Fast Metric Acquisition with Mobile Devices. VMV 2016

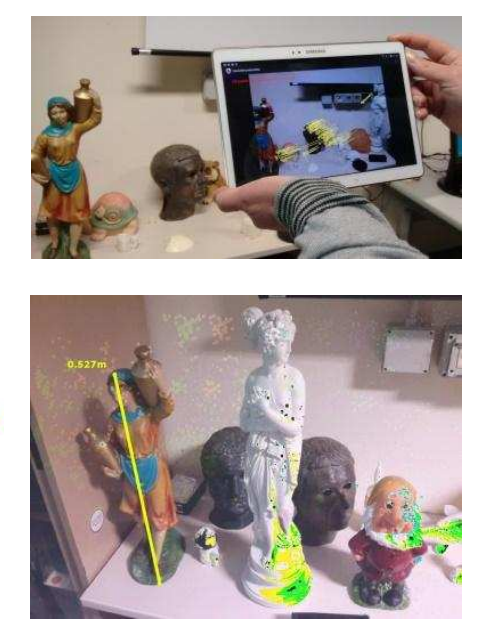




\section{Structure-from-Motion + Dense reconstruction}

- SfM reconstructs a point cloud from a series of images

- 3D positions of (sparse) matched features

- Camera positions and orientations

- Many approaches for densification

- Pipeline showed to work at interactive rates on phones (Taskanen et al 2013)

- SCALE AMBIGUITY
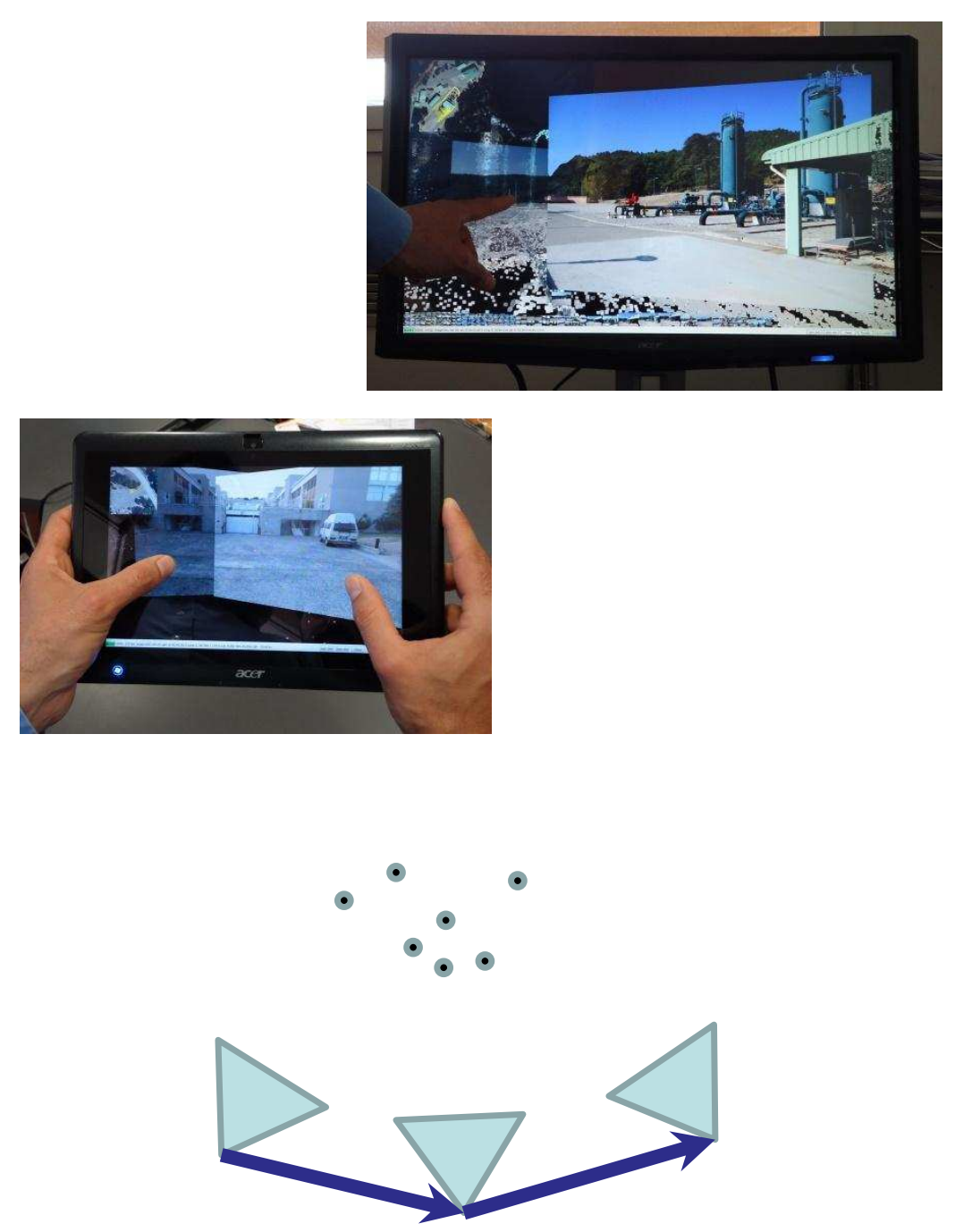


\section{Data fusion: Visual + IMU}

- Use sensors synced with visual channel

- GPS+Magnetometer generally not applicable

- IMU returns orientation and acceleration in real world units

\section{- Idea}

- track camera movement with IMU during visual capture

- use IMU data to find out the real-world distance between SfM camera positions, resolving the scale ambiguity

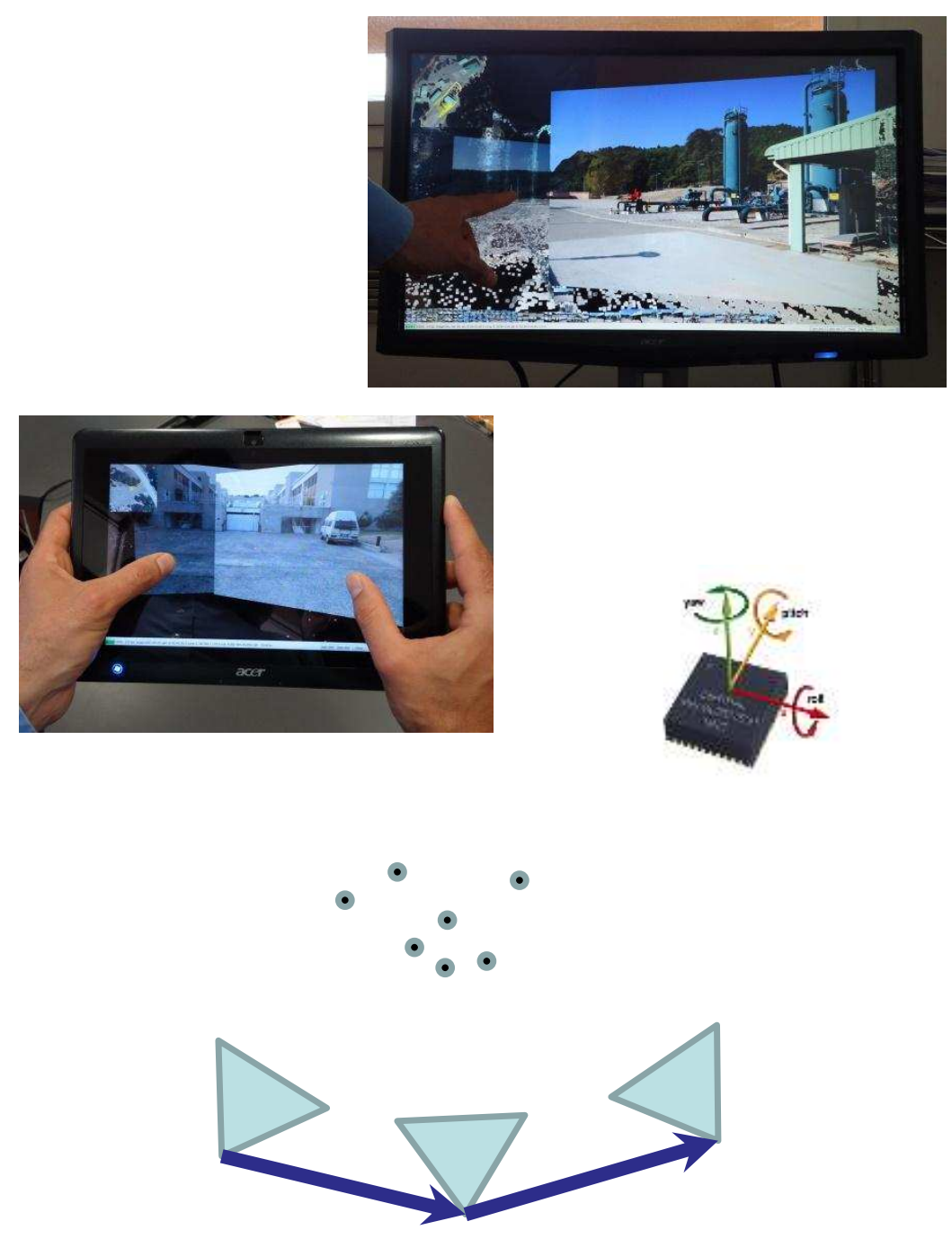




\section{Data fusion: Visual + IMU}

- The accelerometer returns acceleration

- Therefore, we should be able to compute the displacement between two camera positions as

$$
x(T 1, T 2)=\left\|\int_{T 1}^{T 2}\left(v(T 1)+\int_{T 1}^{t \prime} a(t) d t\right) d t^{\prime}\right\|
$$

- Not so easy: onboard IMU sensors are biased and noisy and SfM camera positions are sparse 


\section{Data fusion approaches (1/4)}

- Match position from IMU integration with position from SfM, coping with noise/bias by extensive filtering

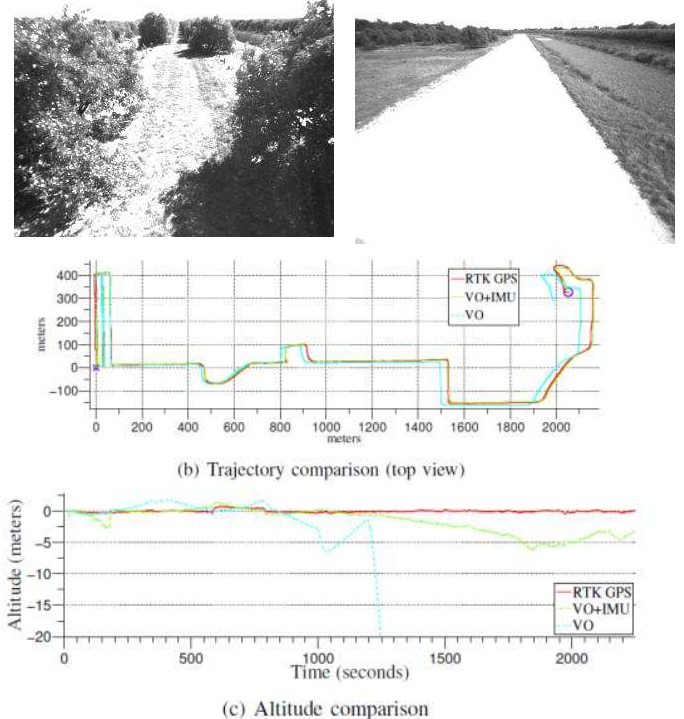

A new approach to vision-aided inertial navigation [Tardif et al 2010]
Vision only IMU only Vision+IMU
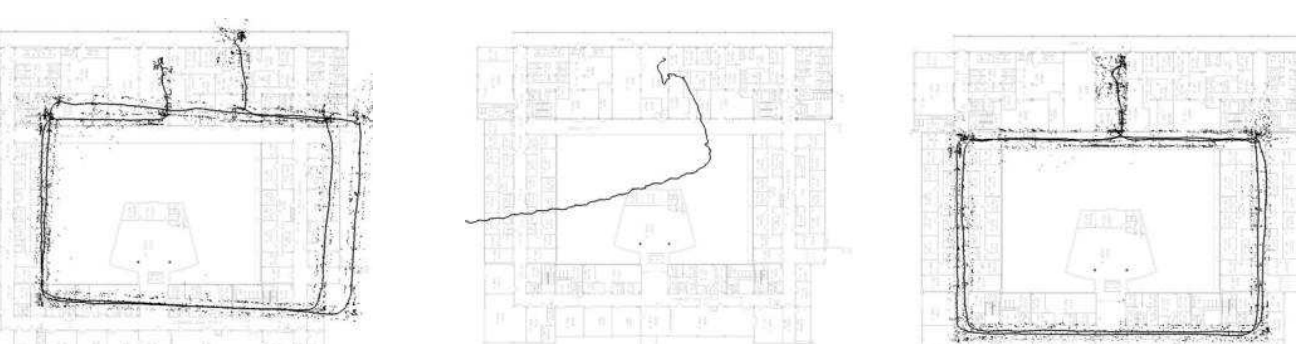

Visual-Inertial Navigation, Mapping and Localization:

A Scalable Real-Time Causal Approach [Jones, Soatto 2011]

- Requires LONG acquisition times and LONG offline processing times 


\section{Data fusion approaches (2/4)}

- Ad-hoc online solutions taking into account IMU characteristics

- Segment motion in "swift movements" with large accelerations

- Integration of IMU acceleration to derive position matched with SfM

- Continuous process of outlier rejection and re-estimation of scale

Live metric 3D reconstruction on

Mobile Phones [Tanskanen et al. 2013]

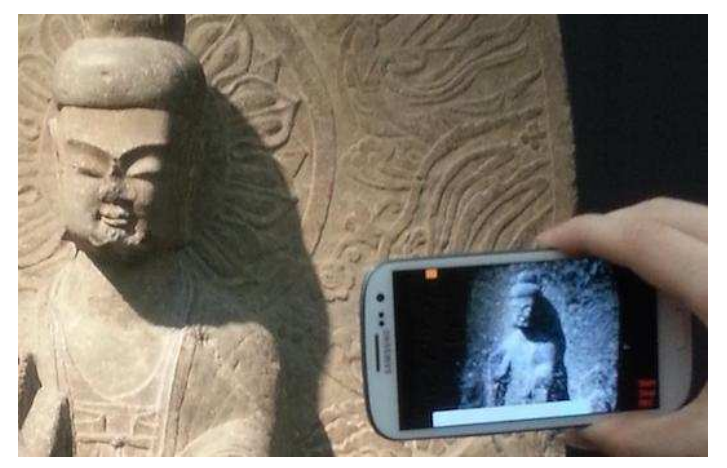

$$
\underset{\lambda}{\arg \min }=\sum_{i \in I}\left\|\vec{x}_{i}-\lambda \vec{y}_{i}\right\|^{2}
$$

One estimate of $\lambda$ at the end of each swift movement Estimation of scale $\lambda$ only on inlier set $I$

- Working but motion-dependent and prone to accumulation error due to integration 


\section{Data fusion approaches (3/4)}

- Match accelerations from IMU with accelerations from SfM at SfM frame-rate (large baseline!)

- Downsample and anti-alias IMU samples at SfM frame rate

- Optimize scale and bias

Hand-waving away scale [Ham et al. 2014]

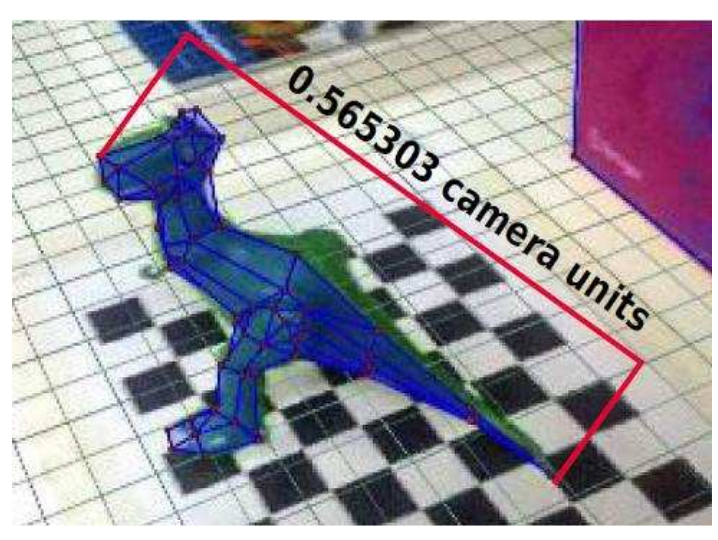

$$
\begin{aligned}
& \underset{s, \mathbf{b}}{\arg \min } \eta\left\{s \cdot \hat{\mathbf{A}}_{V}+\mathbf{1} \otimes \mathbf{b}^{\boldsymbol{\top}}-\mathbf{D} \mathbf{A}_{I} \mathbf{R}_{I}\right\} \\
& \text { D: convolutional matrix for } \\
& \text { antialising and downsampling } \\
& \quad \text { IMU signal }
\end{aligned}
$$

- Requires very long acquisition times due to downsampling at SfM rate 


\section{Data fusion approaches (4/4)}

- Match accelerations from IMU with accelerations from SfM at IMU frame-rate (small baseline!)

- Upsample SfM samples at high rate using all available visual data

- Estimate acceleration from upsampled transforms and match them to IMU samples using robust fitting

Fast Metric Acquisition with Mobile Devices. [Garro et al. 2016\}

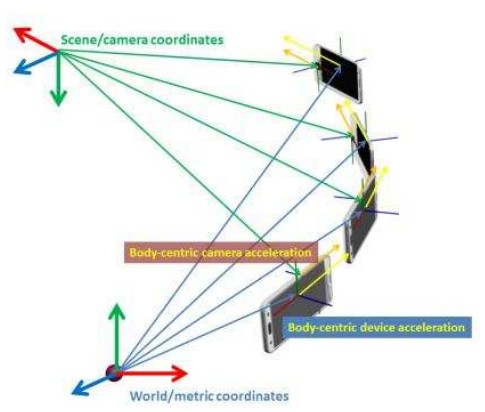

$$
\underset{s, R}{\operatorname{argmin}}\left\{\left\|A_{c}-s R A_{s}\right\|\right\}
$$

- Fast, coping with large errors and noise 


\section{Vision Module Pipeline}

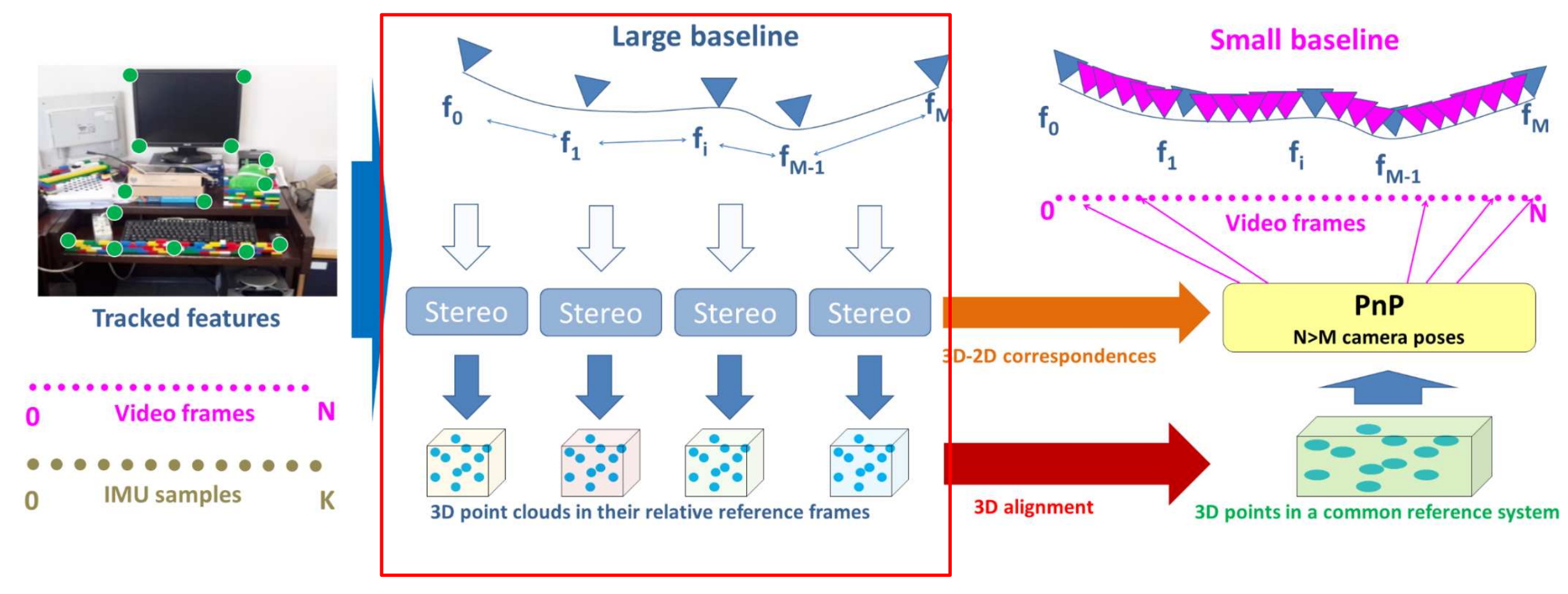




\section{Vision Module}

- Traces Shi-Thomasi features

- When baseline is large enough

- Estimate Essential Matrix, that is, relative camera pose between $\mathrm{f0}$ and fi

- Calculate a 3D point for each feature point

- Note: each pair of cameras has its own reference system

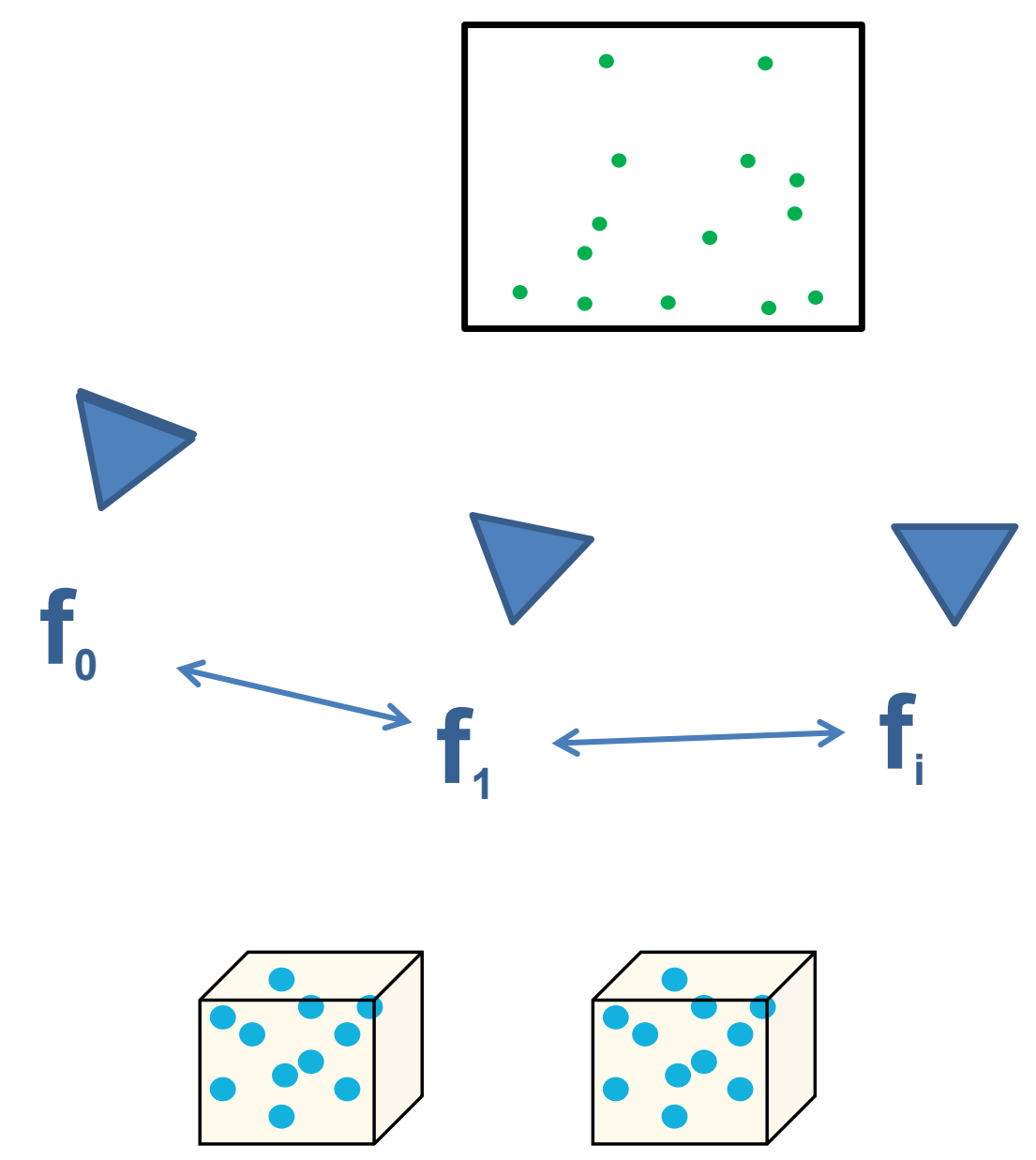




\section{Vision Module}

\section{- Global registration}

- M point clouds

- A subset of features is present in each point cloud

- Use feature correspondence to align all the point cloud in the same reference system

\section{- Cameras upsampling}

- Features are tracked for all frames

- Use aligned point cloud and tracking position to estimate cameras for all frames with Perspective-n-Point (PnP)

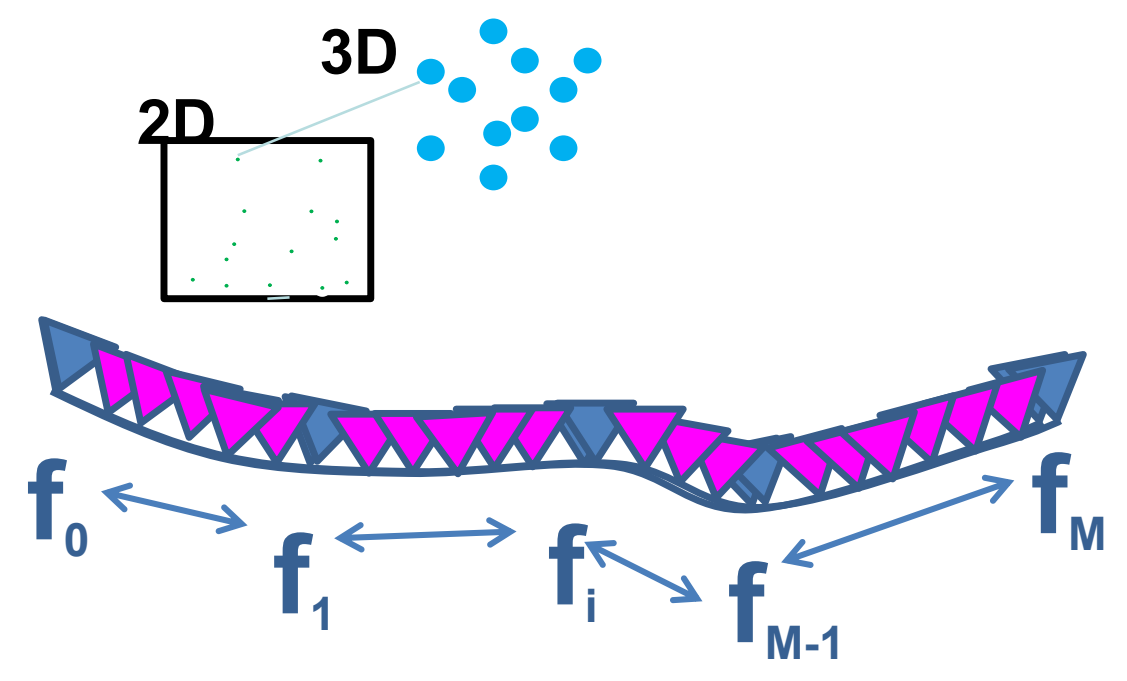




\section{Recovering the scale factor $(1 / 2)$}

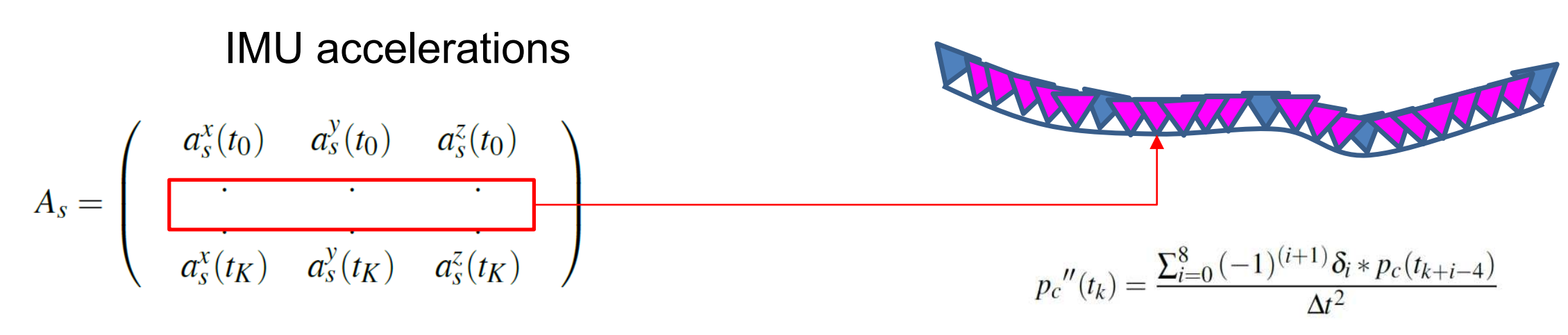

Camera accelerations

$$
A_{c}=\left(\begin{array}{c}
p_{c}{ }^{\prime \prime}\left(t_{0}\right)^{T} R_{c}\left(t_{0}\right) \\
\cdot \\
p_{c}{ }^{\prime \prime}\left(t_{K}\right)^{T} R_{c}\left(t_{K}\right)
\end{array}\right)
$$

Problem to solve

$\underset{s}{\operatorname{argmin}}\left\{\left\|A_{c}-s A_{s}\right\|\right\}$ 


\section{Recovering the scale factor $(2 / 2)$}

- LS, gradient descent (et similia) poorly conditioned

- Not so many data

- Severe outliers

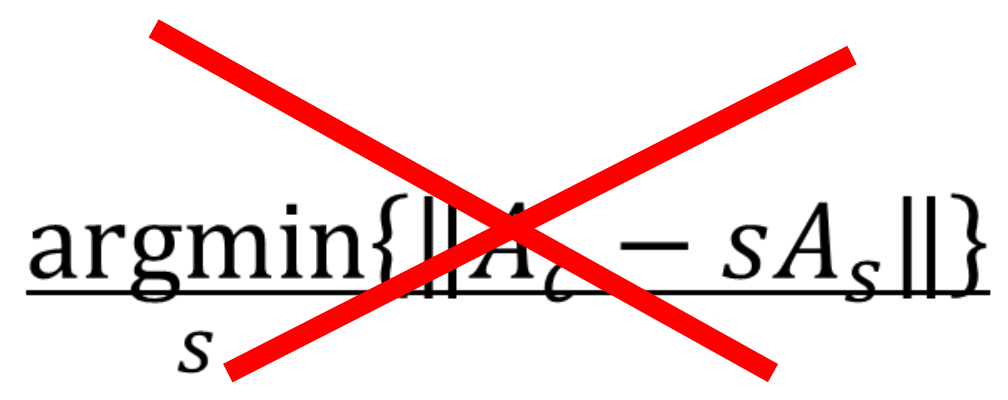

- Robust fitting use RANSAC approach

- Use MLESAC robust estimator to maximize likelihood rather than just the number of inliers

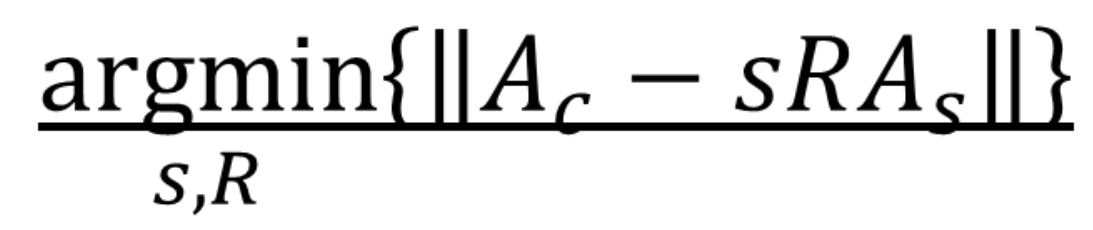

- Introduce rotation matrix $\mathbf{R}$

- Account for orientation bias

- Improve RANSAC performance

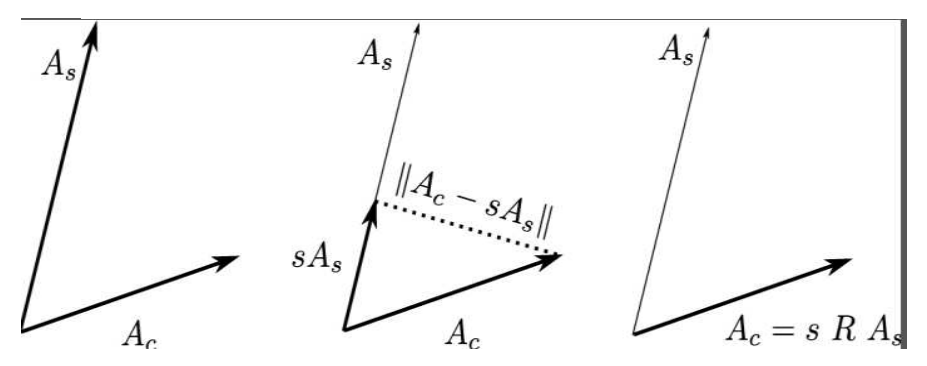




\section{Results}

\begin{tabular}{|c|c|c|c|c|c|c|c|c|}
\hline \multirow{2}{*}{$\begin{array}{l}\text { Scene } \\
\text { Name }\end{array}$} & \multirow{2}{*}{$\begin{array}{c}\text { Real scale } \\
\text { m/s.u. }\end{array}$} & \multicolumn{3}{|c|}{ Acquisition info } & \multicolumn{2}{|c|}{ Our approach } & \multicolumn{2}{|c|}{ Simple scaling } \\
\hline & & Seconds & Poses & Samples & $\mathrm{m} / \mathrm{s} . \mathrm{u}$. & Error & $\mathrm{m} / \mathrm{s.u}$. & Error \\
\hline $3 \mathrm{D}$ printer & 2.094 & 17.0 & 65 & 883 & 2.01 & $4.0 \%$ & 2.85 & $36.1 \%$ \\
\hline Scanner setup & 3.565 & 9.8 & 53 & 641 & 3.45 & $3.1 \%$ & 3.12 & $12.4 \%$ \\
\hline Desktop & 6.520 & 11.3 & 48 & 596 & 6.24 & $4.2 \%$ & 5.16 & $20.8 \%$ \\
\hline Statuettes & 2.602 & 11.5 & 53 & 607 & 2.49 & $4.5 \%$ & 2.48 & $4.9 \%$ \\
\hline Office desk & 1.977 & 30.4 & 88 & 471 & 2.01 & $1.8 \%$ & 2.01 & $1.8 \%$ \\
\hline Office workstation & 3.95 & 12.3 & 37 & 1307 & 3.94 & $0.3 \%$ & 3.98 & $0.6 \%$ \\
\hline Ara pacis & 1.568 & 30.07 & 77 & 1569 & 1.52 & $2.8 \%$ & 1.80 & $13.0 \%$ \\
\hline Workstation (Fastest) & 0.707 & 9.9 & 34 & 1305 & 0.73 & $2.7 \%$ & 0.89 & $20.4 \%$ \\
\hline Desk fast motion & 6.918 & 14.8 & 74 & 1718 & 6.28 & $9.1 \%$ & 3.88 & $44.0 \%$ \\
\hline
\end{tabular}

- Median error $\mathbf{4 \%}$ (wrt $\mathbf{1 0 - 1 5 \%}$ of other STAR solutions) 


\section{Example 2}

\section{DATA FUSION AND COMMUNICATION FOR INDOOR CAPTURE}




\section{Indoor capture + presentation}

- Creation and sharing of indoor digital mock-ups

- Exploiting the capabilities of modern mobile devices
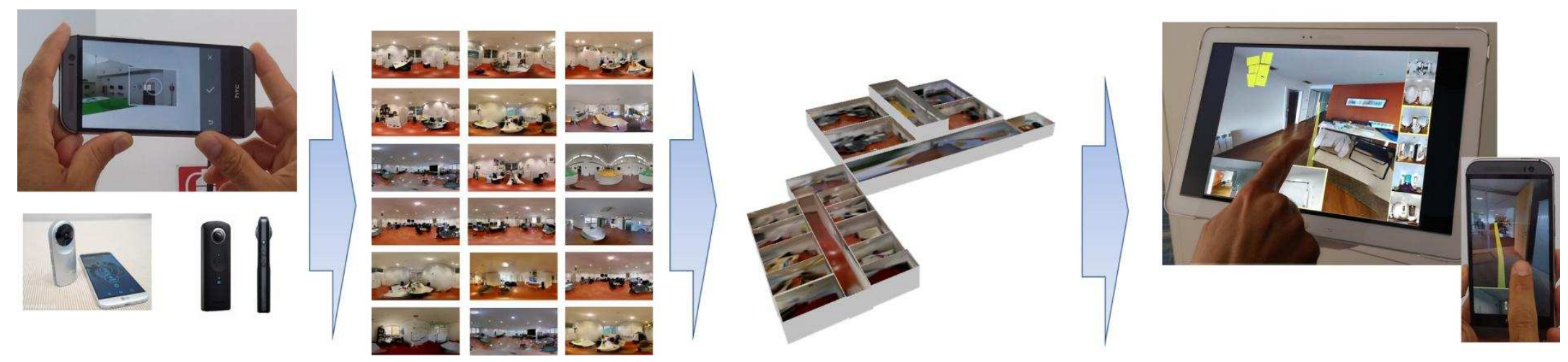

- Much interest/applications (security, location awareness, ...)

- Need to capture visual information together with room structure 


\section{Typical solutions}

- Indoor capture and modeling

- Manual modeling

- Semi-automatic methods based on high-density data

- Laser scanning

- Professional but expensive, limited to specific applications

- Multi-view stereo from photographs

- Generally cost effective but hard to apply in the indoor environment

"Walls poorly textured, occlusions, clutter

" Furthermore: need for heavy MW constraints, computationally demanding
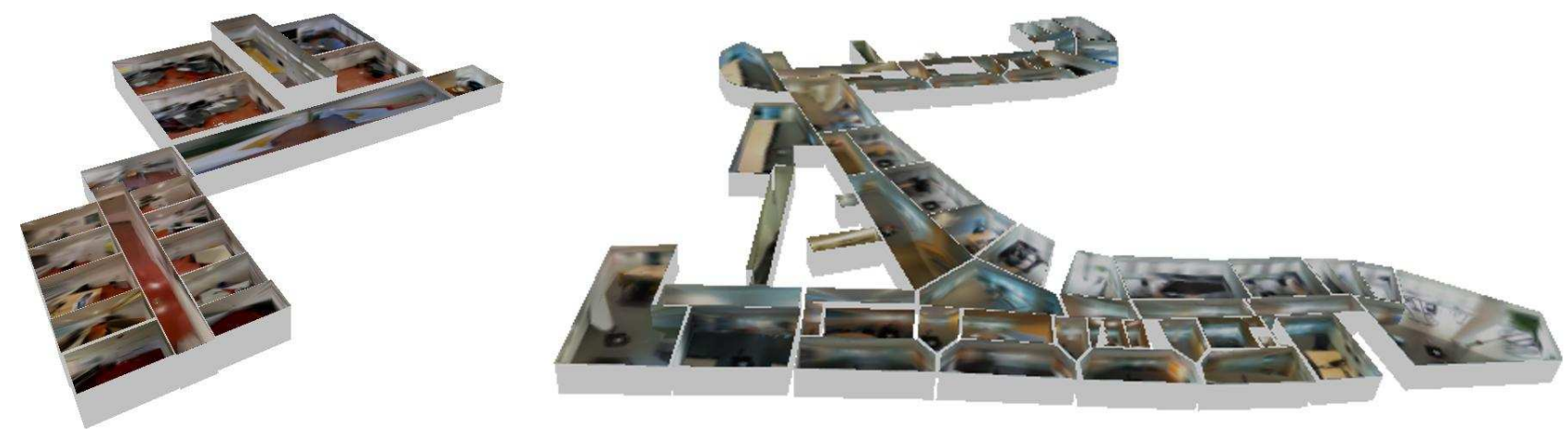


\section{Examples using low-cost mobile devices}

- Interactive capture and mapping of indoor environment

- MagicPlan - http://www.sensopia.com

- Floor corners marked via an augmented reality interface

- Manual editing of the room and floor plan merging using the screen interface

- Sankar and Seitz: Capturing indoor scenes with smartphones (UIST2012)

- Corners marked on the screen during video playback

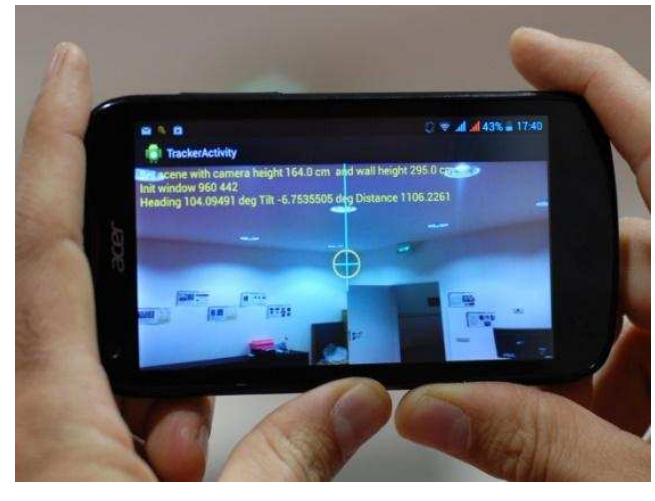

Pintore et al. Effective mobile mapping of multi-room indoor structures The Visual Computer,2014
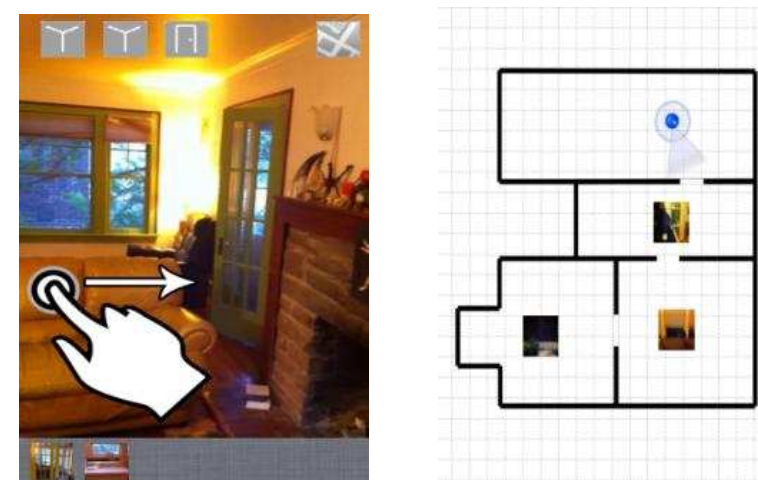

Sankar et al. Capturing indoor scenes with smartphones, UIST2012 


\section{Exploiting panoramic images}

- 360 degrees images are easy to capture using common devices

- Interactive apps using IMU + GUI + automatic stitching

- Dedicated cameras

- 360 degrees images are easy to navigate

- Spheremaps + emerging formats video+image formats

- VR devices for immersion

- What about analyzing them?
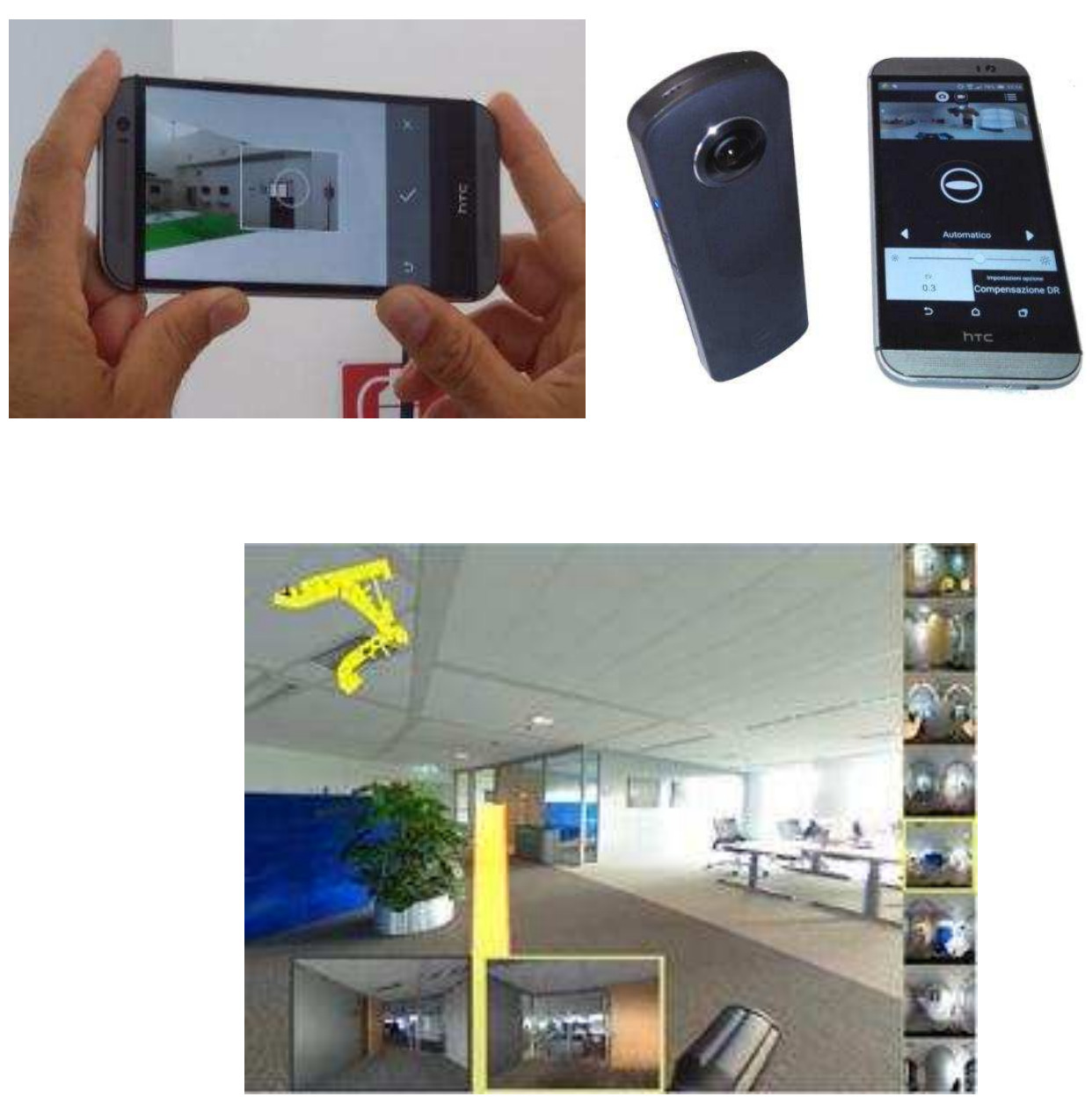


\section{Finding the room structure}

- Take one spheremap per room

- Equirectangular images generated by a mobile device

- Vertical lines aligned with the gravity vector

- Image approx. oriented towards magnetic North

- Eventually use IMU + Visual features for stitching

- Track user motion to identify connections between rooms

- Use IMU + Visual Features for tracking

- Solve local + global optimization to find indoor structure

- Multi-room environment
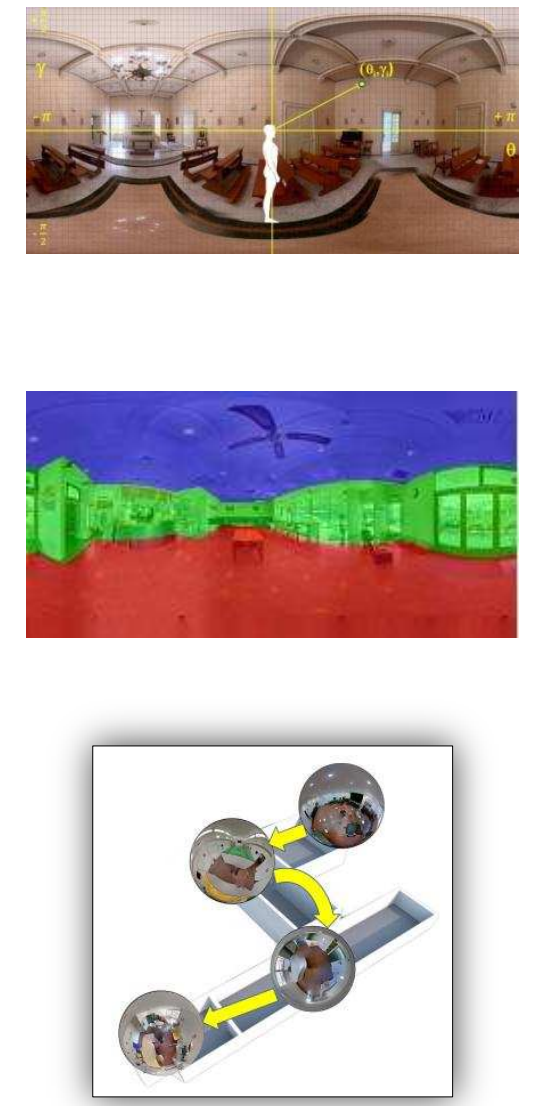


\section{Finding the room structure}

- Analyze spheremap to extract single room structure

- Room model considers vertical walls

- Extract edges and filter out regions likely far from top/bottom edges of walls

- Find wall height

- Voting scheme used to extract most likely wall height by maximizing pairs of matching wall-floor / wallheight edge pixels

- Fit 2.5D room model to recovered wall edge map

- Uses specialized transform to speed-up computation
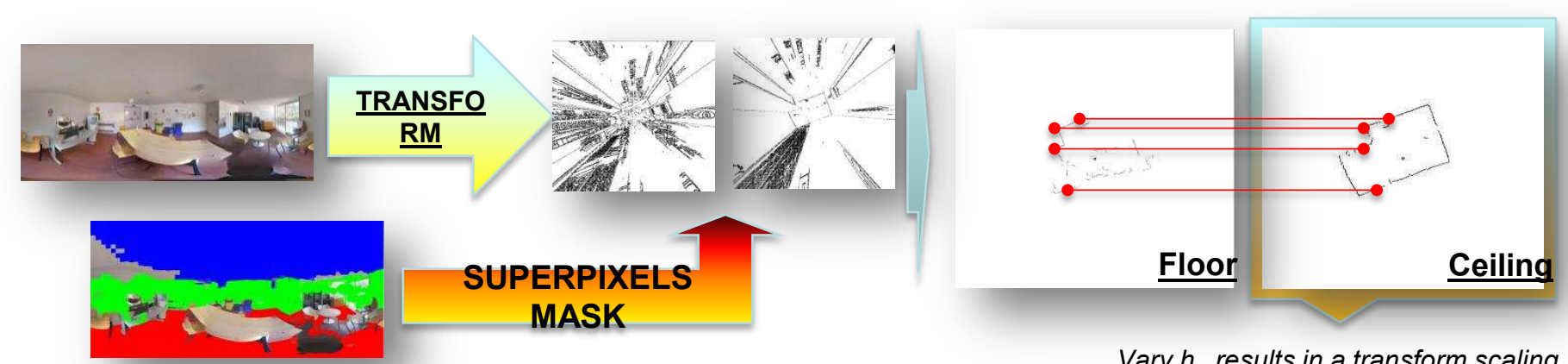


\section{Finding the rooms structure}

\section{- Iterated to map the entire floor-plan}

- Mobile tracking of user's direction moving between adjacent rooms creates a connected room graph

- Doors position identification in the image by computer vision

- Doors matching according with graph

- Rooms displacement

- Global optimization of combined model
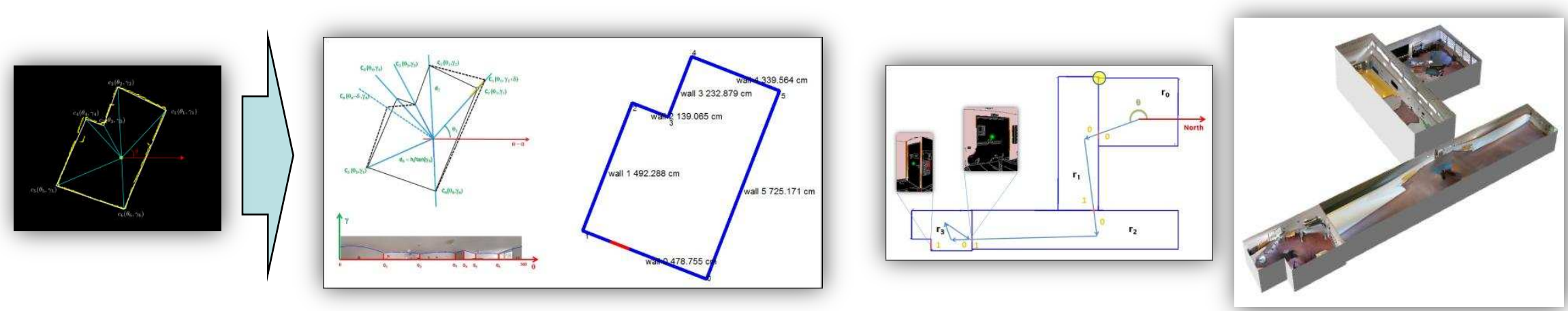

Pintore et al. Omnidirectional image capture on mobile devices for fast automatic generation of 2.5D indoor maps. IEEE WACV 2016 


\section{Results}
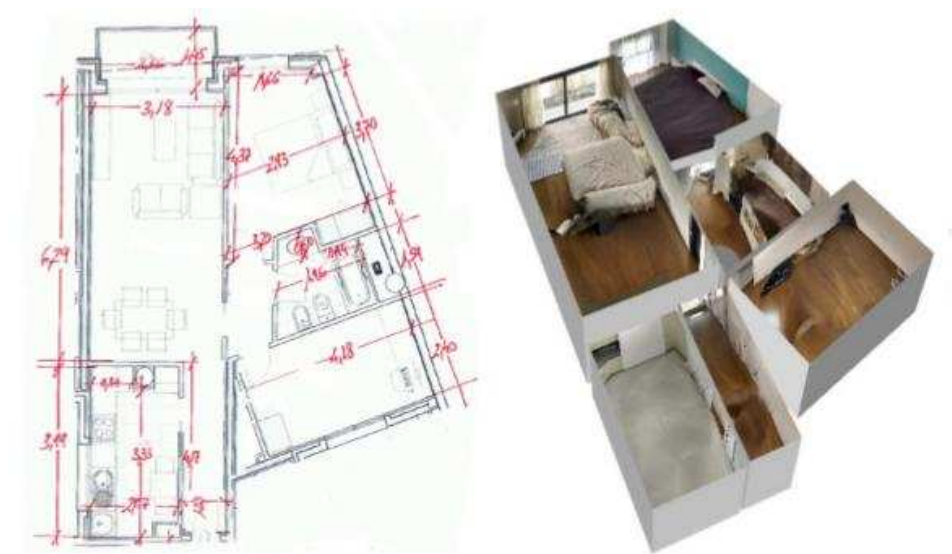

\begin{tabular}{|c|cc|cc|cc|cc|cc|c|}
\hline $\begin{array}{c}\text { Scene } \\
\text { Name }\end{array}$ & \multicolumn{2}{|c|}{ Features } & \multicolumn{2}{|c|}{ Area error } & \multicolumn{2}{|c|}{ Wall length error } & \multicolumn{2}{|c|}{ Wall height error } & \multicolumn{2}{c|}{ Corner angle error } & Editing time \\
Area $\left[\boldsymbol{m}^{2}\right]$ & Np & MP & Ours & MP & Ours & MP & Ours & MP & Ours & MagicPlan \\
\hline Office H1 & 720 & 10 & $2.95 \%$ & $1.78 \%$ & $35 \mathrm{~cm}$ & $15 \mathrm{~cm}$ & $2.0 \mathrm{~cm}$ & $1.2 \mathrm{~cm}$ & $0.8 \mathrm{deg}$ & $0.8 \mathrm{deg}$ & $26 \mathrm{~m} 32 \mathrm{~s}$ \\
Building B2 & 875 & 25 & $2.50 \%$ & $1.54 \%$ & $30 \mathrm{~cm}$ & $7 \mathrm{~cm}$ & $6.0 \mathrm{~cm}$ & $1.5 \mathrm{~cm}$ & $1.5 \mathrm{deg}$ & $1.5 \mathrm{deg}$ & $42 \mathrm{~m} 18 \mathrm{~s}$ \\
Commercial & 220 & 6 & $2.30 \%$ & $1.82 \%$ & $25 \mathrm{~cm}$ & $8 \mathrm{~cm}$ & $12.0 \mathrm{~cm}$ & $2.7 \mathrm{~cm}$ & $1.5 \mathrm{deg}$ & $1.0 \mathrm{deg}$ & $28 \mathrm{~m} 05 \mathrm{~s}$ \\
Palace & 183 & 3 & $16.86 \%$ & $0.20 \%$ & $94 \mathrm{~cm}$ & $5 \mathrm{~cm}$ & $45.0 \mathrm{~cm}$ & $1.3 \mathrm{~cm}$ & $1.8 \mathrm{deg}$ & $0.5 \mathrm{deg}$ & $15 \mathrm{~m} 08 \mathrm{~s}$ \\
House 1 & 55 & 5 & $21.48 \%$ & $2.10 \%$ & $120 \mathrm{~cm}$ & $16 \mathrm{~cm}$ & $15.0 \mathrm{~cm}$ & $4.7 \mathrm{~cm}$ & $13.7 \mathrm{deg}$ & $1.2 \mathrm{deg}$ & $25 \mathrm{~m} 48 \mathrm{~s}$ \\
House 2 & 64 & 7 & $28.05 \%$ & $1.67 \%$ & $85 \mathrm{~cm}$ & $8 \mathrm{~cm}$ & $18.0 \mathrm{~cm}$ & $3.5 \mathrm{~cm}$ & $15.0 \mathrm{deg}$ & $0.5 \mathrm{deg}$ & $32 \mathrm{~m} 25 \mathrm{~s}$ \\
House 3 & 170 & 8 & $25.10 \%$ & $2.06 \%$ & $115 \mathrm{~cm}$ & $15 \mathrm{~cm}$ & $20.0 \mathrm{~cm}$ & $4.0 \mathrm{~cm}$ & $18.0 \mathrm{deg}$ & $1.5 \mathrm{deg}$ & $29 \mathrm{~m} 12 \mathrm{~s}$ \\
\hline
\end{tabular}

Pintore et al. Omnidirectional image capture on mobile devices for fast automatic generation of 2.5D indoor maps. IEEE WACV 2016

\section{- Reasonable, fast reconstruction with rough structure and visual features}




\section{Sharing the indoor model}

- Indoor model

- Exploration graph

- Each node is a spheremap/room

- edges (yellow) are transitions between adjacent rooms

- Stored on a server (standard http Apache2)

- Panoramic images

- Mapped according with the graph

\section{- Interactive exploration}

- Room

- WebGL fragment shader

- dragging to change view orientation and pinching to zoom in/out

- Passages

- Real-time rendering of the transitions between rooms

Exploiting geometric model stored on the server

- Performance improvement compared to use precomputed videos

- Suggested paths
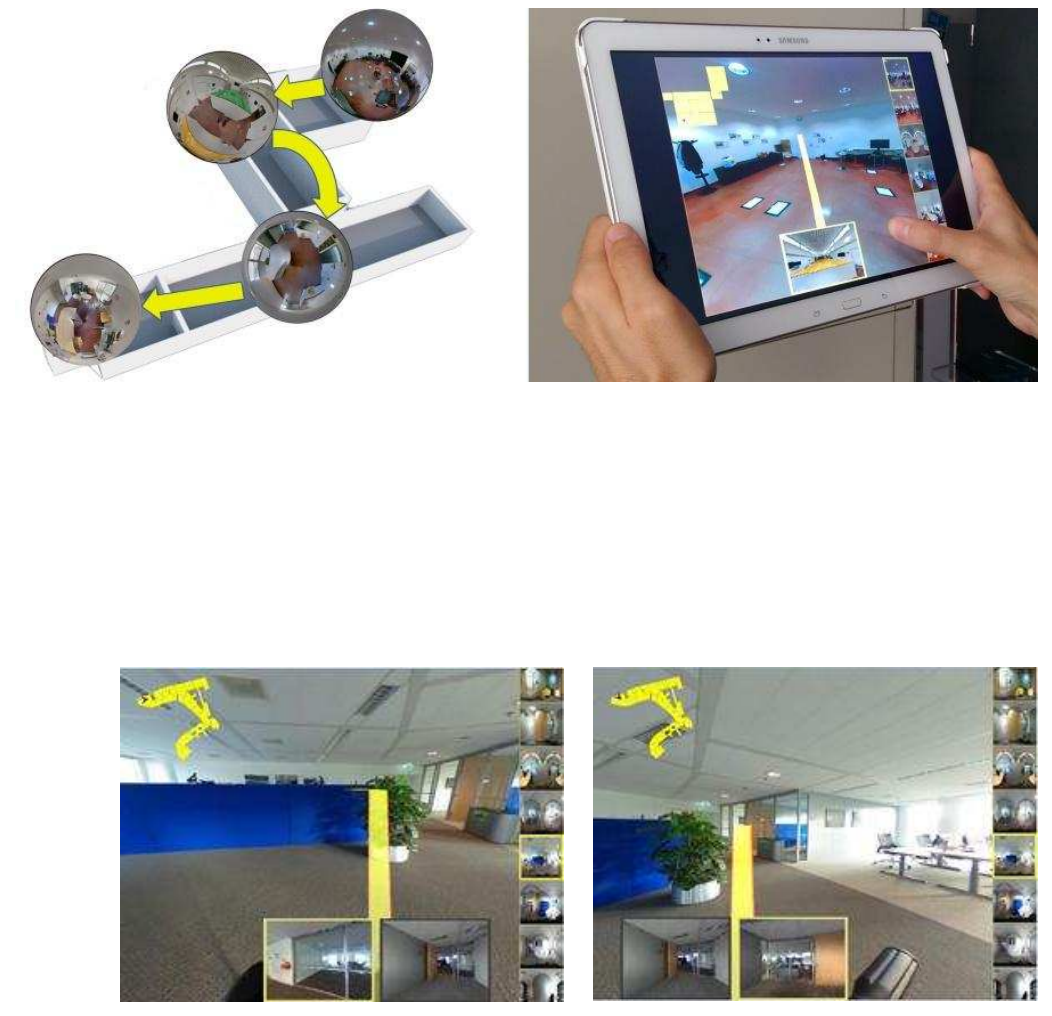


\section{Some results}

\section{Live dlemo: http://vcg.isti.cnr.it/vasco/ Click on the dataset on the left column to start}
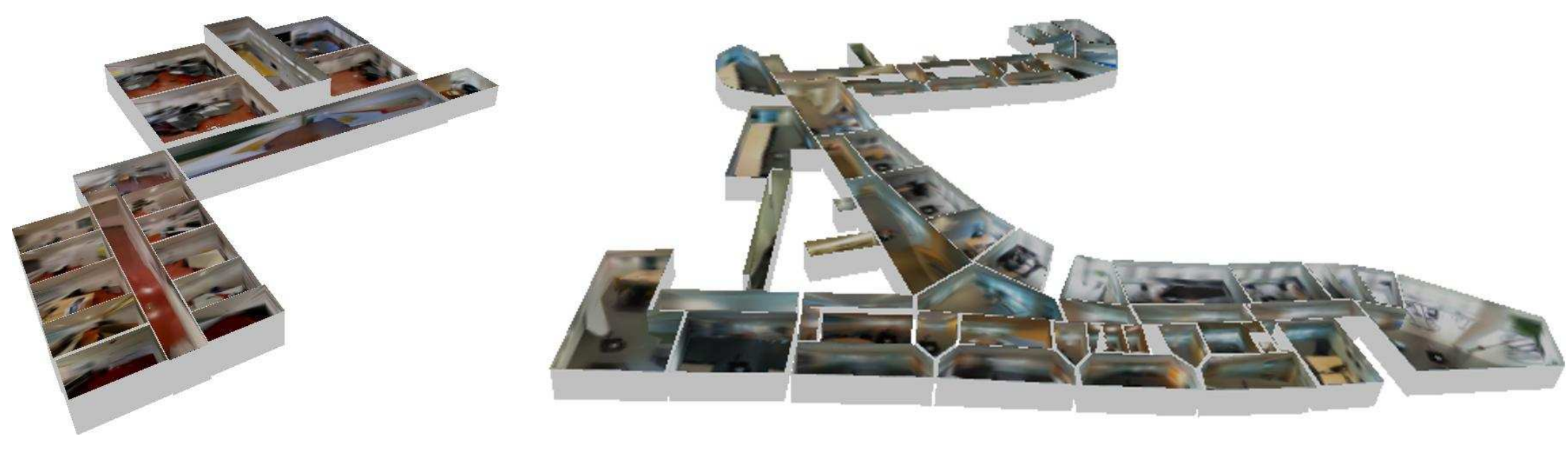

3D reconstruction of a $655 \mathrm{mq}$ office with 19 rooms. This environment was acquired with a mobile phone (HTC One M8)
Reconstruction of a 70 rooms floor of the NHV ministry at Den Haag, Netherlands. The whole model was acquired with a Ricoh Theta S camera 
Next session:

CLOSING/Q\&A 


\section{Part 6}

\section{All good things come to an end...}

(Bad ones, too) 


\section{Subject: Mobile Graphics}

- All you need to know to get an introduction to the field of mobile graphics:

- Scope and definition of "mobile graphics"

- Brief overview of current trends in terms of available hardware architectures and research apps built of top of them

- Quick overview of development environments

- Rendering, with focus on rendering massive/complex surface and volume models

- Capture, with focus on data fusion techniques 


\section{Contacts (in alphabetical order)}

- Marco Agus $(1,2)$

- Research Engineer at KAUST (Saudi Arabia)

- Researcher at CRS4 (Italy)

- Enrico Gobbetti (1) - organizer

- Director of Visual Computing at CRS4 (Italy)

- Fabio Marton (1)

- Researcher at CRS4

- Giovanni Pintore (1)

- Researcher at CRS4

- Pere-Pau Vázquez (3)

- Professor at UPC, Spain

\section{(1) www.crs4.it/vic/}

\section{(2) https://vcc.kaust.edu.sa}

\section{(3) http://www.virvig.eu/}




\section{Funding...}

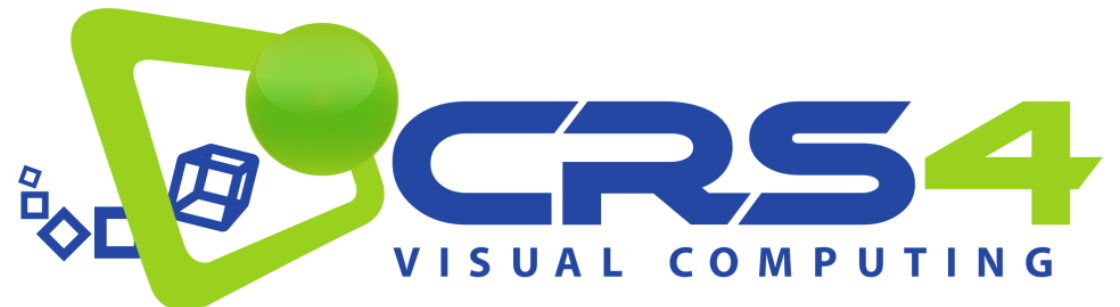

Center for Research, Development, and Advanced Studies in Sardinia, Italy

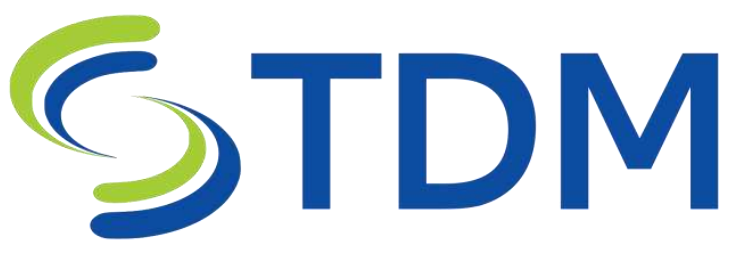

Project TDM RAS - POR FESR 2014-2020

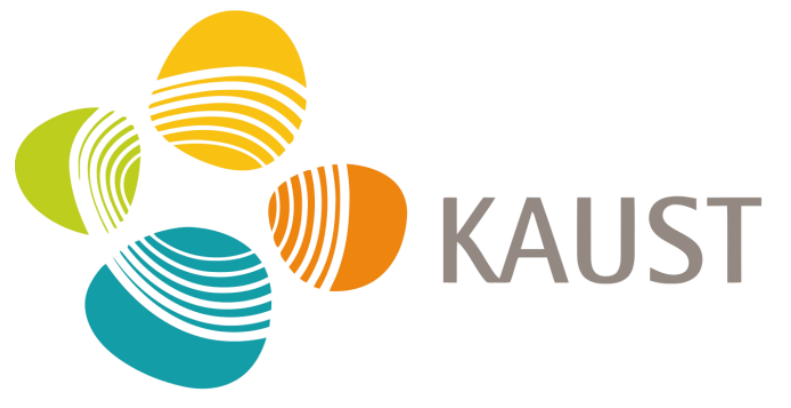

King Abdullah University of Science \& Technology, Saudi Arabia

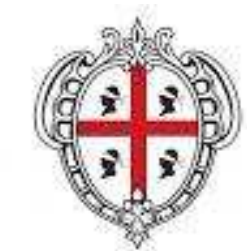

REGIONE AUTONOMA DE SARDIGNA REGIONE AUTONOMA DELLA SARDEGNA Projects VIGEC / VIDEOLAB

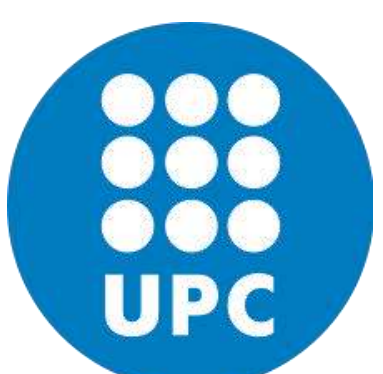

Polytechnic University of Catalonia, Spain 
Thanks for your attention! Q\&A NOW (TIME PERMITTING...) 


\section{More information...}

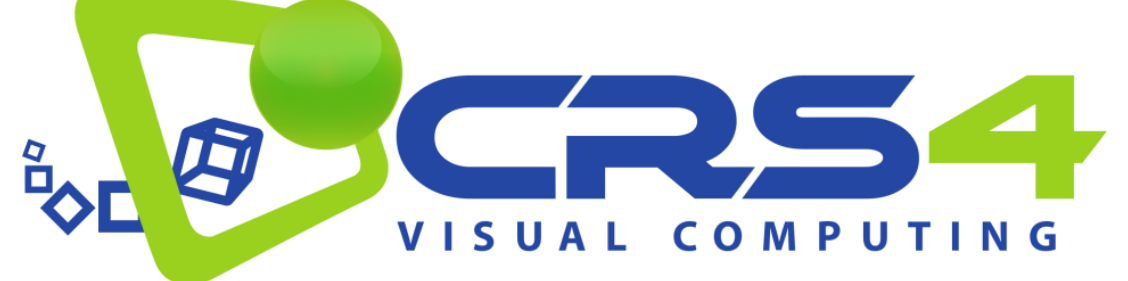

Center for Research, Development, and Advanced Studies in Sardinia, Italy

www.crs4.it/vic/

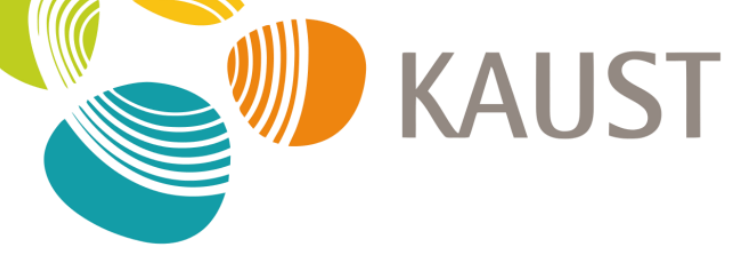

King Abdullah University of Science \& Technology, Saudi Arabia

vcc.kaust.edu.sa

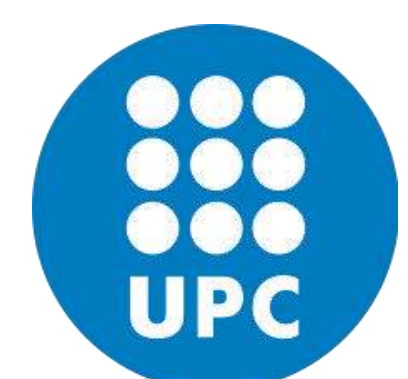

Polytechnic University of Catalonia, Spain

www.virvig.eu 